\title{
Science on High-Energy Lasers: From Today to the NIF
}

\author{
Richard W. Lee, \\ Richard Petrasso, \\ Roger W. Falcone
}

\section{January 1995}
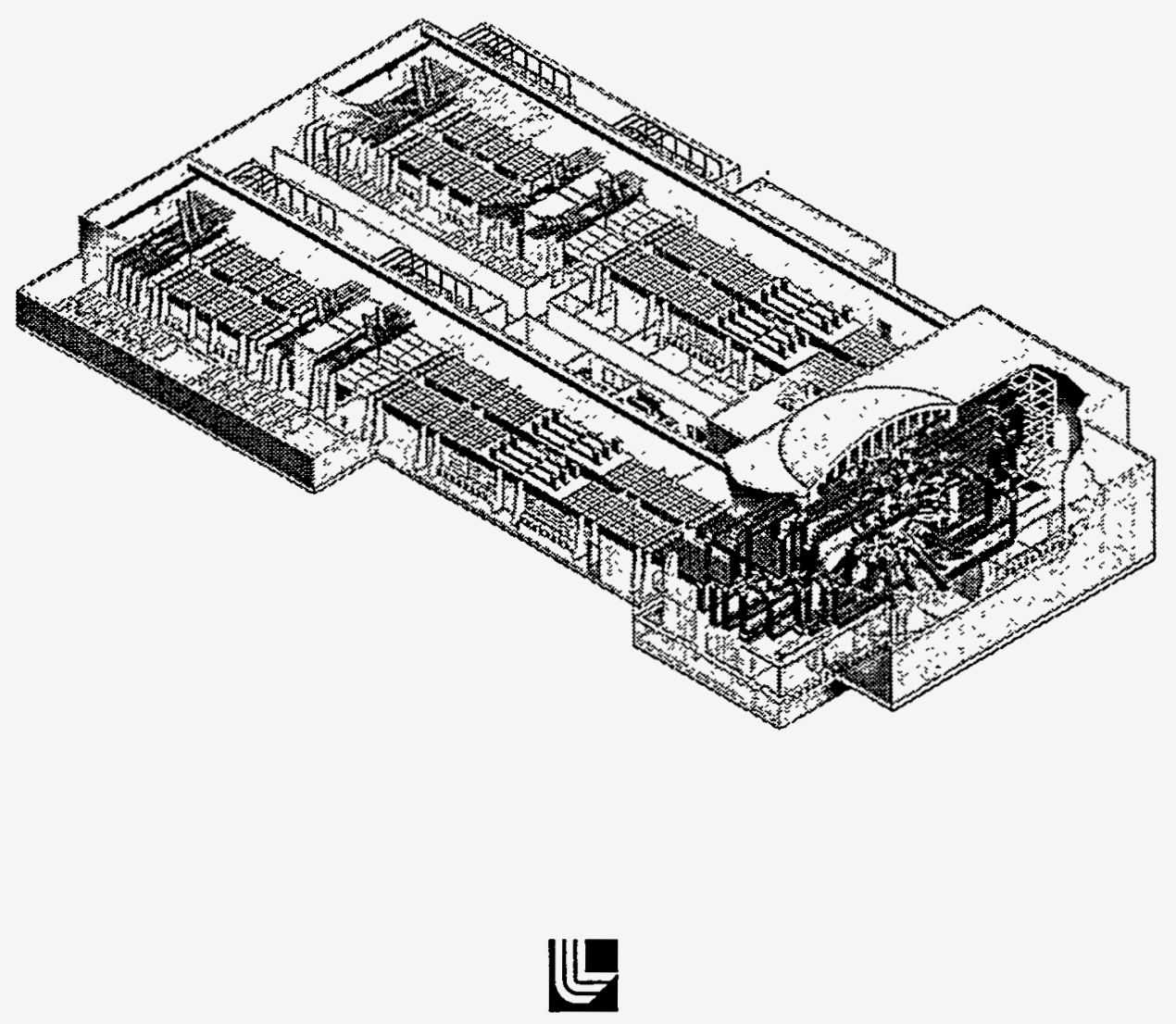

LAWRENCE LIVERMORE NATIONAL LABORATORY University of California • Livermore, California $\bullet 94550$ 


\section{DISCLAIMER}

This document was prepared as an account of work sponsored by an agency of the United States Government. Neither the United States Government nor the University of California nor any of their employees, makes any warranty, express or implied, or assumes any legal liability or responsibility for the accuracy, completeness, or usefulness of any information, apparatus, product, or process disclosed, or represents that its use would not infringe privately owned rights. Reference herein to any specific commercial products, process, or service by trade name, trademark, manufacturer, or otherwise, does not necessarily constitute or imply its endorsement, recommendation, or favoring by the United States Government or the University of California. The views and opinions of authors expressed herein do not necessarily state or reflect those of the United States Government or the University of California, and shall not be used for advertising or product endorsement purposes.

This report has been reproduced directly from the best available copy.

Available to DOE and DOE contractors from the

Office of Scientific and Technical Information P.O. Box 62, Oak Ridge, TN 37831

Prices available from (615) 576-8401, FTS 626-8401

Available to the public from the

National Technical Information Service

U.S. Department of Commerce

5285 Port Royal Rd.,

Springfield, VA 22161

This is an informal report intended primarily for internal or limited external distribution. The opinions and conclusions stated are those of the author and may or may not be those of the Laboratory.

Work performed under the auspices of the U.S. Department of Energy by Lawrence Livermore National Laboratory under Contract W-7405-Eng-48. 


\section{DISCLAIMER}

Portions of this document may be illegible in electronic image products. Images are produced from the best available original document. 


\section{Science on High-Energy Lasers:}

From Today to the NIF

Richard W. Lee,

Richard Petrasso,

Roger W. Falcone

January 1995 
UCRL-ID-119170

This page intentionally left blank 


\section{Table of Contents}

Acknowledgments .......................................................................................................................................ix

Introduction ................................................................................................................................................

Section I-High-Energy Lasers in the Context of Current Science ......................................... I

Section II-Overview of Science on High-Energy Lasers....................................................... 3

A. Astrophysics and Space Physics .......................................................................... 3

B. Hydrodynamics ......................................................................................................... 4

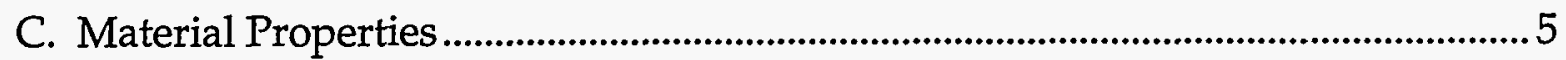

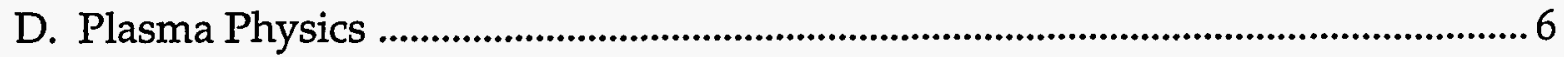

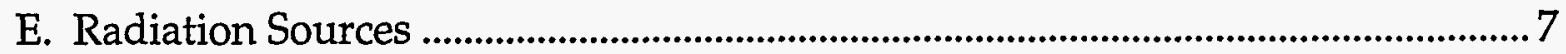

F. Radiative Properties ..................................................................................................... 8

Section III-Experimental Capabilities .......................................................................................... 9

A. Definition of Existing Facility ....................................................................................... 9

The Laser................................................................................................................................................

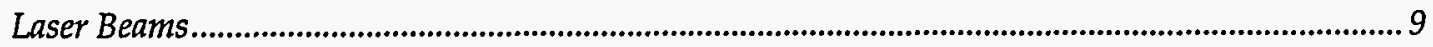

Beam Pointing, Aiming, and Synchronicity .............................................................................. 10

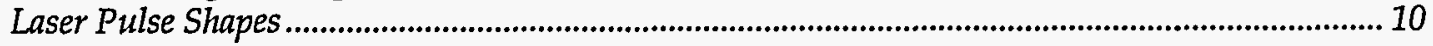

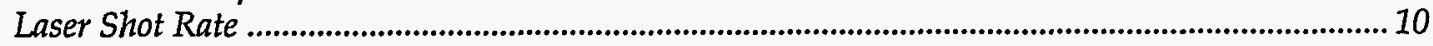

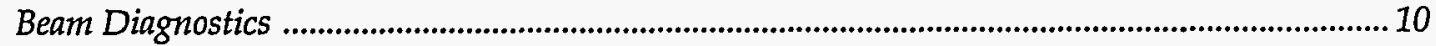

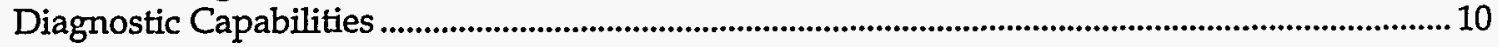

Overview of Target Chamber Diagnostics .............................................................................. 11

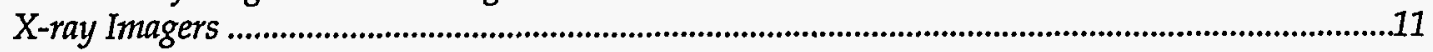

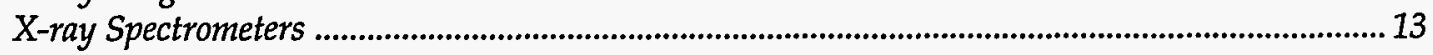

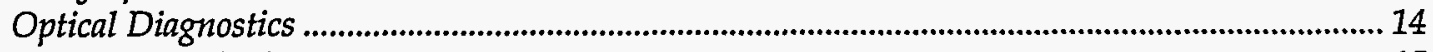

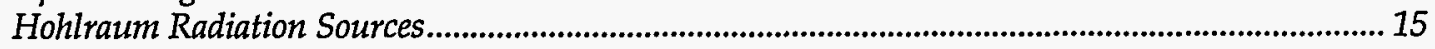

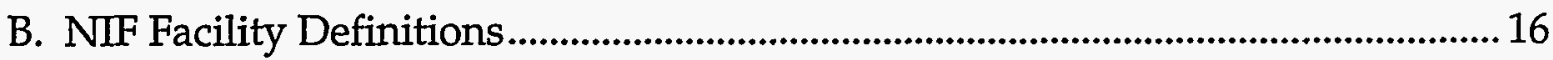

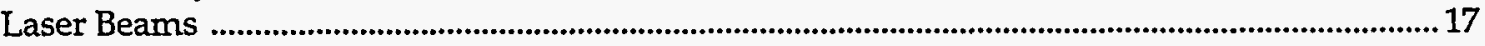

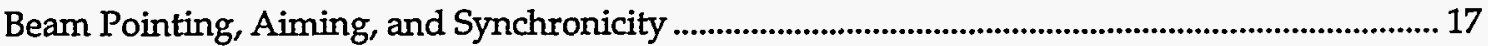

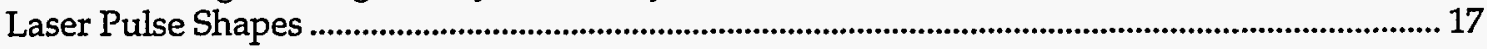

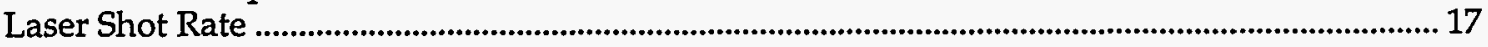

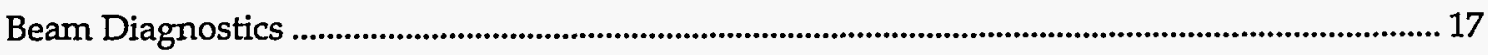

C. NIF Facility Modifications …................................................................................... 18

Higher Beam Irradiance Requirements ............................................................................... 18

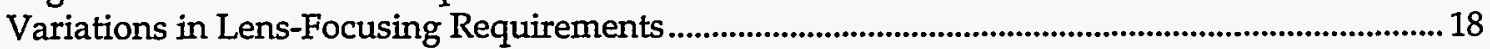

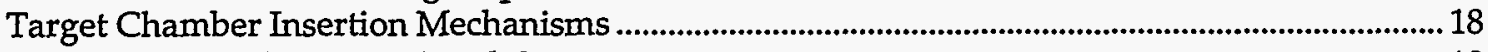

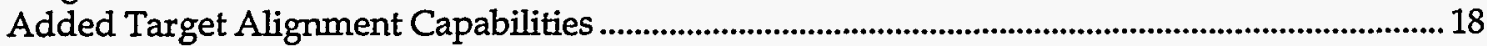

Reduced Time Between Experiments ................................................................................................ 18

Flexible Time Intervals Between Experiments............................................................................... 19

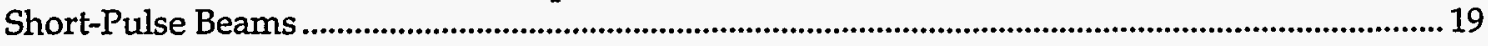

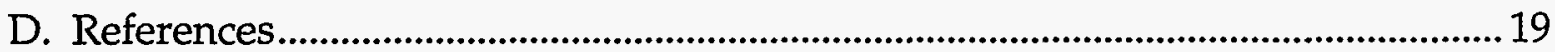


Section IV-Astrophysics and Space Physics ........................................................... 21

A. Opacity

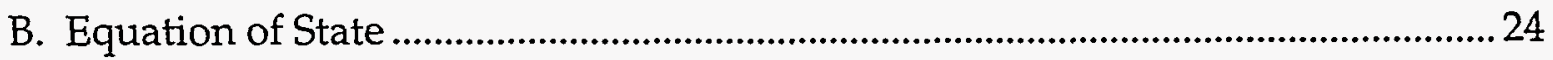

C. Plasma Spectroscopy in High-Energy Astrophysics ...............................................26

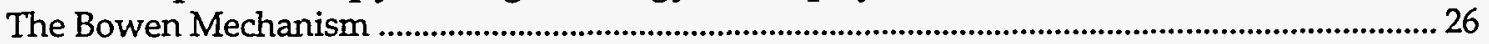

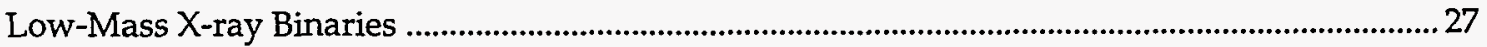

Shock-Wave Ionized Media ................................................................................................................2 29

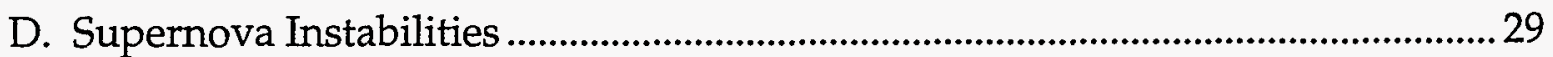

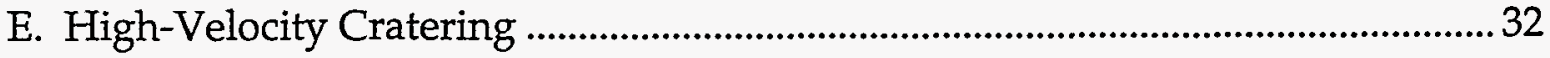

F. Thermonuclear Reaction Rates in Stars ...............................................................

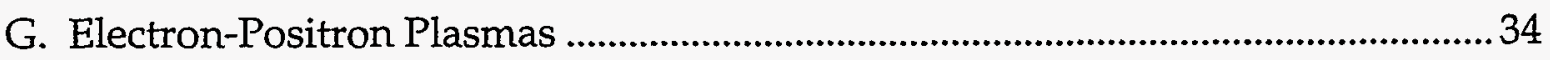

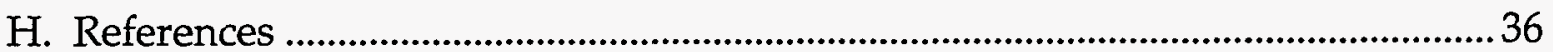

Section V-Hydrodynamics............................................................................................................. 37

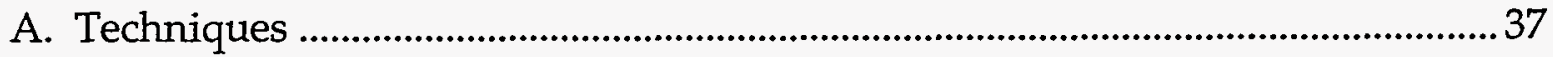

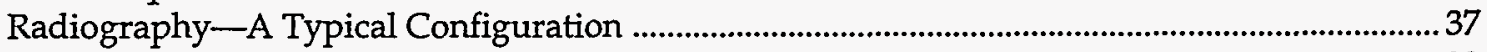

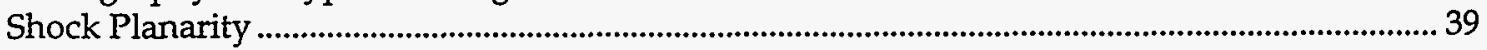

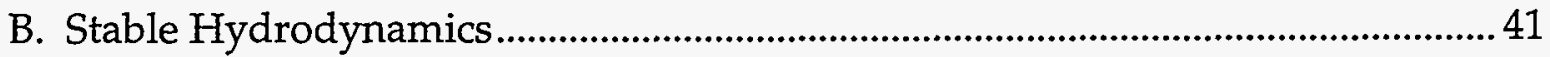

Planar Geometry .................................................................................................................................... 41

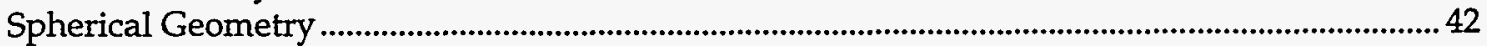

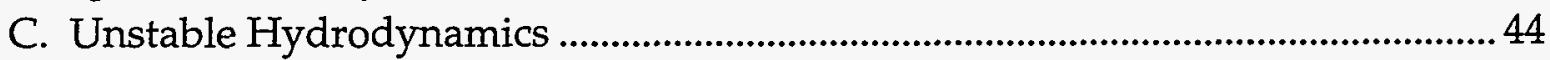

Planar Geometry .................................................................................................................................... 44

Imposed Perturbation-Rayleigh-Taylor .......................................................................................4 44

Imbedded Random Surface-Rayleigh-Taylor ...............................................................................4 46

Imbedded Interface - Richtmyer-Meshkov .........................................................................................49

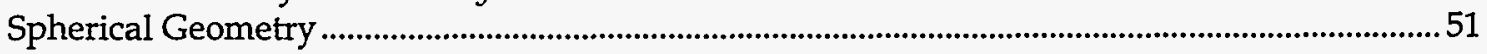

D. Future NIF Experiments ……..........................................................................54

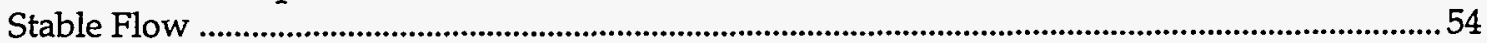

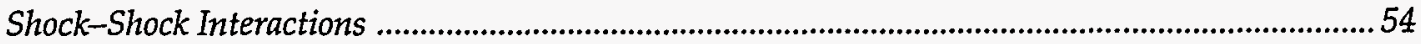

Shock-Boundary Interactions ..........................................................................................................5

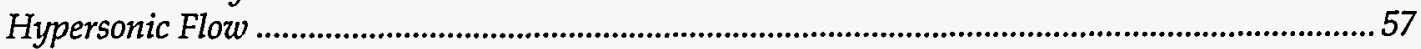

Impact Cratering ...........................................................................................................................5

Scaled Radiative Energy Coupling .......................................................................................................58

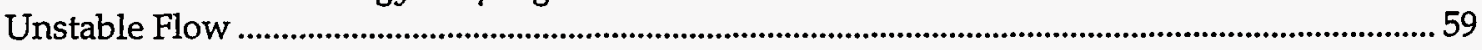

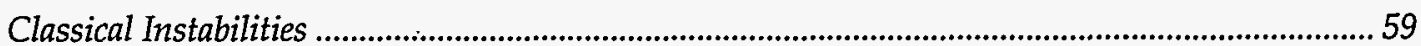

Other Instabilities ................................................................................................................

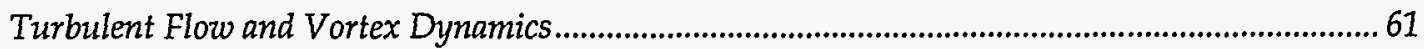

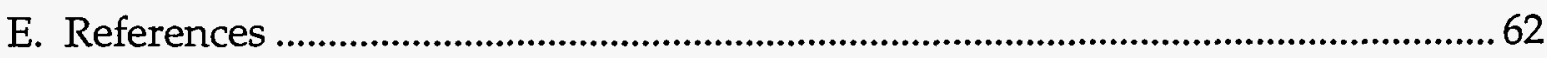

Section VI-Material Properties ...................................................................................................6 63

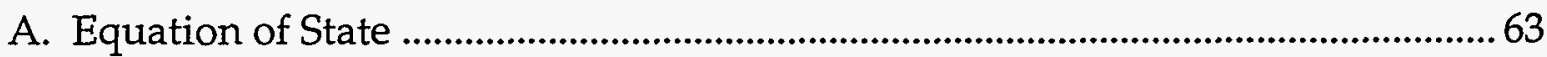

Directly Driven High-Pressure Shocks ...............................................................................................6

Indirectly Driven Colliding Foil Experiments.....................................................................................64

Indirectly Driven Shock Experiments on Polystyrene ....................................................................67

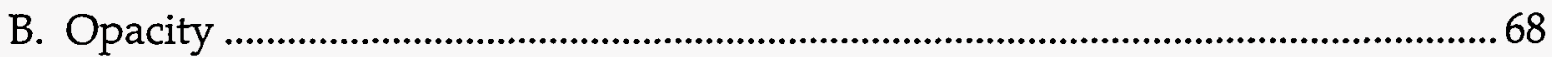




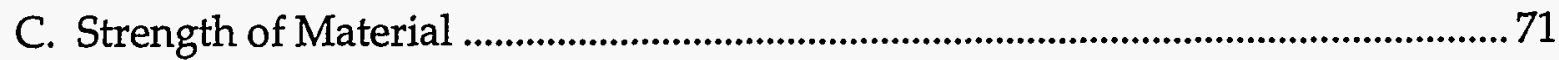

Simple Compression Studies ...............................................................................................................72

Plasticity and Adiabatic Shear Bands ...................................................................................................72

Metastable Phases ......................................................................................................................................... 73

Phase-Change Measurements ...............................................................................................................73

Rear-Surface Studies ................................................................................................................................

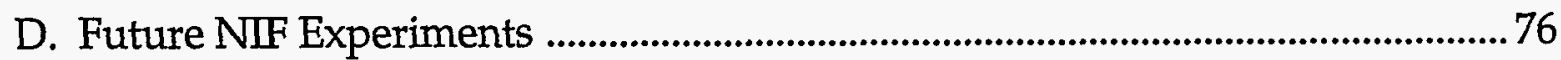

The Principal Hugoniot ........................................................................................................................ 77

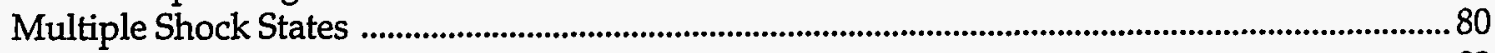

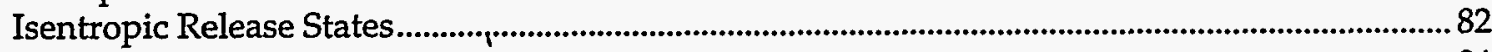

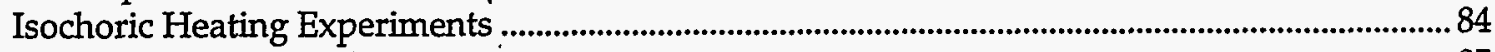

X-ray Diffraction Studies ........................................................................................................................... 85

Other Material Properties.......................................................................................................................... 87

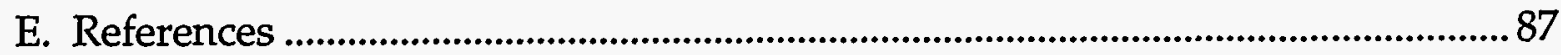

Section VII-Plasma Physics .............................................................................................................. 89

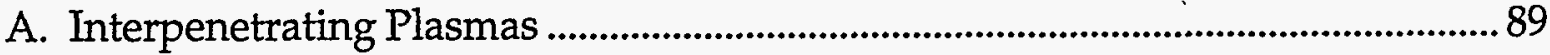

B. Plasma Streaming in Magnetic Fields.....................................................................91

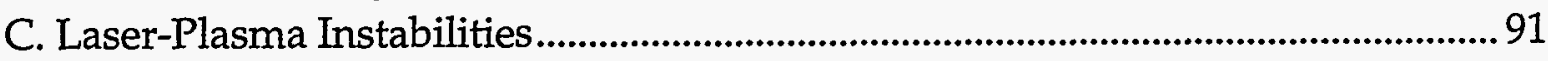

Stimulated Raman Scattering ………………............................................................................................. 93

Stimulated Brillouin Scattering …………............................................................................................94

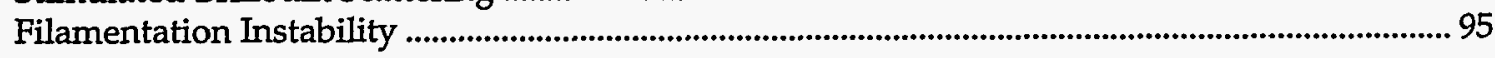

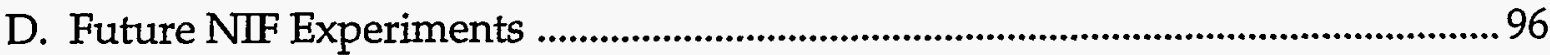

Laser-Plasma Interactions in Large, Hot Plasmas ..........................................................................97

Long Scale-Length Plasma Production ................................................................................................97

Stimulated Brillouin and Raman Scattering ................................................................................... 98

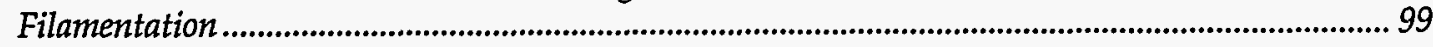

Short-Pulse High-Intensity Laser-Plasma Interactions..................................................................... 100

Study of Very High Magnetic Fields ....................................................................................................... 101

Generation of Large Fluxes of .......................................................................................................... 102

Interpenetrating Plasmas and Turbulence..........................................................................................102

Nuclear Reactions in Large-Volume, High-Temperature Plasmas.................................................. 102

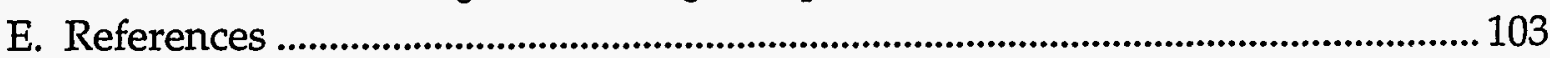

Section VIII-Radiation Sources ........................................................................................ 107

A. Spectrally Continuous Sources ................................................................................107

Conversion Efficiency of Gold.Burn-Through Foils.........................................................................107

Spectral Character of Gold Burn-Through Foils ................................................................................107

Temporal Behavior of Gold Burn-Through Foils ..............................................................................109

Spectral Character of Other Foils ..................................................................................................... 109

Angle of Incidence vs Conversion Efficiency …………………………...........................................112

Laser Intensity vs Conversion Efficiency .....................................................................................114

Hohlraum Radiation Temperature Scaling ……………………….....................................................114

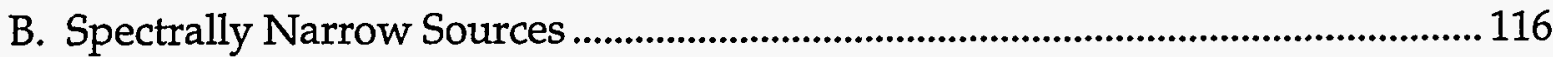

Effect of $Z$ on Emission ............................................................................................................................116

Comments on Sparseness of Data ........................................................................................................117 


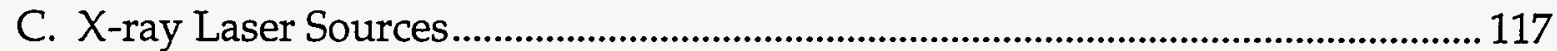

Studying X-ray Lasers...............................................................................................................117

Two-dimensional Plasma Imaging ....................................................................................................118

Development of $X$-ray Lasers as Probes........................................................................................121

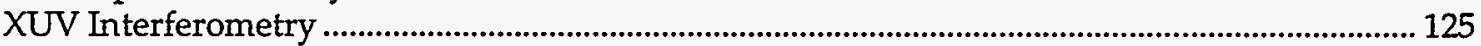

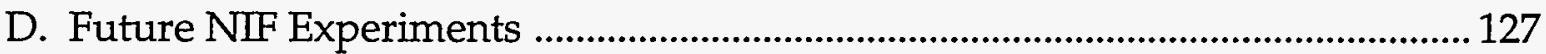

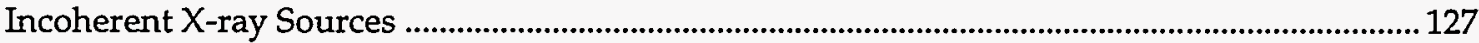

Broadband Sources.................................................................................................................... 127

Narrow or Line Sources ............................................................................................................... 128

Coherent X-ray Sources .....................................................................................................................128

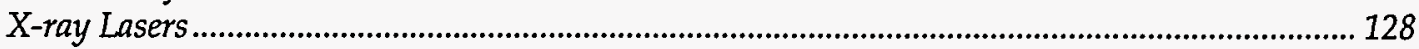

High-Order Harmonic Generation .............................................................................................130

Applications of X-ray Lasers................................................................................................... 131

Particle Sources ................................................................................................................................. 131

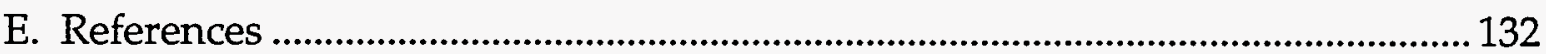

Section IX-Radiative Properties..................................................................................................... 133

A. Atomic Physics and Isolated-Emitter Spectroscopy ............................................... 133

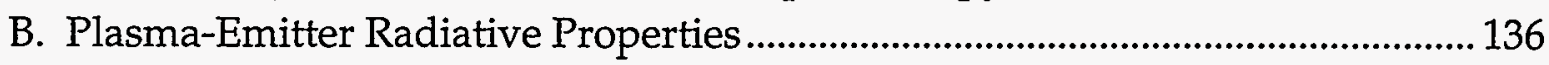

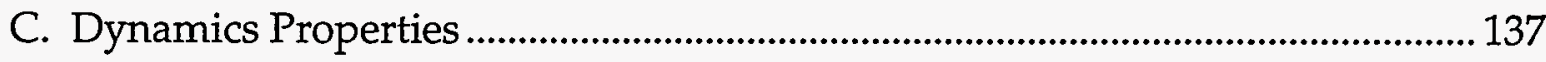

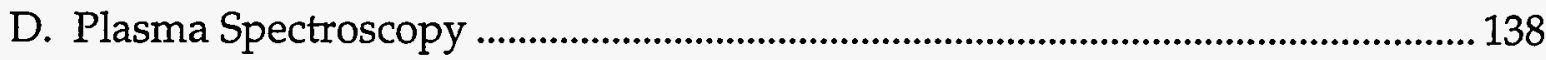

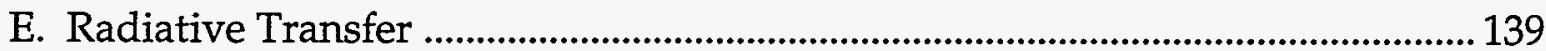

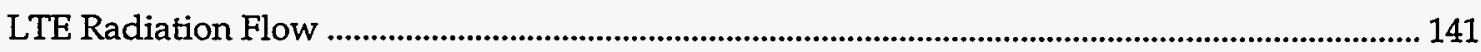

Non-LTE Radiative Transfer ....................................................................................................... 142

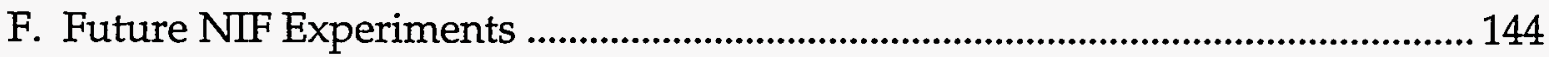

Spectroscopy of High-Z Elements...................................................................................................144

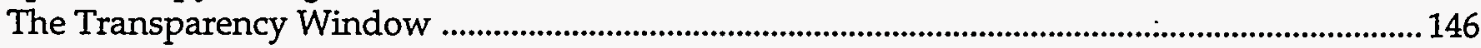

Strongly Correlated Effects ............................................................................................................... 149

Plasma Spectroscopic Topics ...................................................................................................153

Spectral Line Shifts, Level Shifts, and Continuum Lowering ......................................................... 153

Ion Dynamics.......................................................................................................................... 155

Continuum Measurements ..............................................................................................................156

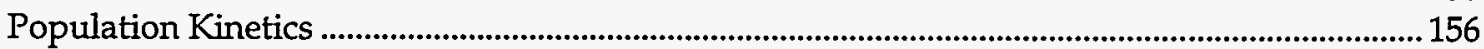

Radiation Transfer and Line Formation ...................................................................................157

Strong Magnetic Field Effects.......................................................................................................... 159

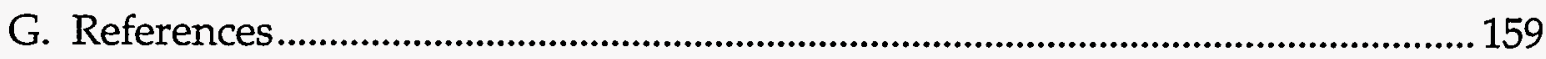




\section{Figures and Tables}

\section{Section III-Experimental Capabilities}

Figure III-1. Schematic view of a hohlraum .

Figure III-2. Schematic view of several different hohlraums .........................................................15

Figure III-3. Measured radiation temperature vs input laser power .............................................16

Table III-1. Diagnostics available on Nova, with their abbreviated names ...............................12

\section{Section IV-Astrophysics and Space Physics}

Figure IV-1. Comparison of the regimes of temperature and density ..........................................2

Figure IV-2. Variation of temperature and density with depth for models of stellar structure

Figure IV-3. Effects of improved opacity and equations of state on understanding Cepheid pulsations

Figure IV-4. Grotrian diagram for the Bowen mechanism ..........................................................26

Figure IV-5. Illustration of the process of dielectronic recombination.........................................28

Figure IV-6. Rayleigh-Taylor mix in supernova modeling ..........................................................30

Figure IV-7. Schematic setup of an astrophysical mix experiment...............................................31

Figure IV-8. Reaction rates that form the CNO bi-cycle.....................................................................34

Figure IV-9. Trajectories in temperature and density parameter space for some big-bang nucleosynthesis models.....................................................................................................35

\section{Section V-Hydrodynamics}

Figure V-1. Side view of the jet experimental arrangement ............................................................37

Figure V-2. Microscope photographs of the cylindrical carbon foam piece...............................38

Figure V-3. Comparison of experimental data with simulation at $t=19.5 \mathrm{~ns}$ (early time).......39

Figure V-4. Comparison of experimental data with simulation at $\mathrm{t}=25.8 \mathrm{~ns}$ (late time) .........39

Figure V-5. Schematic of a shock breakout experiment .................................................................39

Figure V-6. Two candidate mounting schemes to study planarity ...........................................40

Figure V-7. Breakout data for the two different mounting schemes, standard and tophat.....40

Figure V-8. Schematic of a test bed for hydrodynamic simulations............................................41

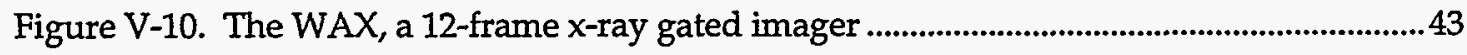

Figure V-11. Typical implosion sequence for a 1-ns square drive pulse......................................43

Figure V-12. Time history of an implosion.................................................................................44

Figure V-13. Schematic of a setup to study large-growth Rayleigh-Taylor instability ..............45 
Figure V-14. Face-on streak-camera image of the backlight source absorbed by the foil .........46

Figure V-15. Intensity traces for the accelerated foil.......................................................................46

Figure V-16. Diagram of target for study of imbedded random surface ......................................46

Figure V-17. Schematic of point projection spectroscopy technique ............................................47

Figure V-18. Spectrometer images from point projection spectroscopy .......................................48

Figure V-19. Radiograph of a sulfinated plastic ablator driving a molybdenum foil, above, with three intensity traces ............................................................................................4

Figure V-20. Axial distribution of mass areal densities ...............................................................50

Figure V-21. Gated $x$-ray image of planar Richtmyer-Meshkov mix target .............................. 50

Figure V-22. A 100- $\mu \mathrm{m}$-wide vertical trace of the centerline of the image in Fig. V-21........... 50

Figure V-23. Vertical trace of the image in Fig. V-21 normalized by the backlight....................51

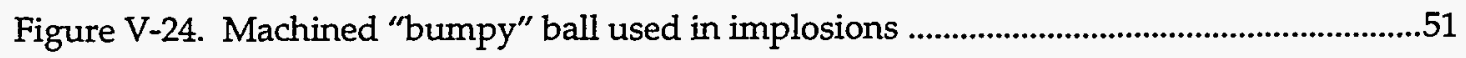

Figure V-25. Expanded view of an implosion image taken using the WAX...............................52

Figure V-26. Measured trace quantifying the limb brightening seen in Fig. V-25 ....................52

Figure V-28. Streak camera spectra from a microsphere with an iron dopant ..........................53

Figure V-29. Schematic of a shock-shock interaction experiment ...............................................55

Figure V-30. Graph of electron density vs time in $\mu$ s for two cases...............................................55

Figure V-31. Propagation of a shock in an inhomogeneous medium ..........................................56

Figure V-32. Setup to explore the hydrodynamic response of a simulated soil .........................57

Figure V-33. Experimental results of hydrodynamic response of soil ..........................................58

Figure V-35. The results of a simulation of the generation of vortices in an incompressible flow

\section{Section VI-Material Properties}

Figure VI-1. Schematic of direct laser-driven shock experiment ..................................................64

Figure VI-2. Streak image of direct laser-driven shock experiment .............................................65

Figure VI-3. Schematic of radiation-driven shock using a 2-step target foil ...............................65

Figure VI-4. Streak camera image of radiation-driven shock using a 2-step target ....................66

Figure VI-5. Schematic setup with target foil shielded and stepped ...........................................67

Figure VI-6. Overview of arrangement for radiation-driven shock experiments .....................68

Figure VI-7. Schematic of point projection spectroscopy method for measuring opacity .......69

Figure VI-8. Absorption (opacity) of the aluminum/niobium sample .........................................70

Figure VI-9. Schematic of $x$-ray diffraction technique .....................................................................72

Figure VI-10. Schematic of a rear-surface shock breakout experiment .........................................74

Figure VI-11. Data from the rear-surface shock breakout experiment..........................................75

Figure VI-12. Spectra from the probed shocked rear surface of a crystal ...................................75

Figure VI-13. Plot of stress vs strain rate domain .........................................................................77

Figure VI-14. The principal Hugoniot defined ..............................................................................78

Figure VI-15. Relationships of the Hugoniot, an isotherm, and an isentrope.............................78

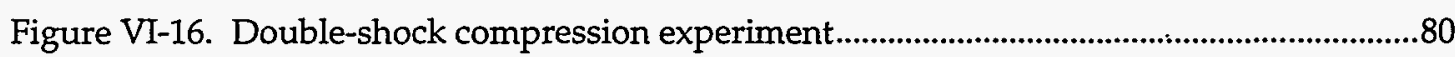

Figure VI-17. Schematic of double-shock compression experiments ........................................... 81 
Figure VI-18. Model of pressure history vs time …….............................................................. 82

Figure VI-19. Model of pressure history vs volume.....................................................................83

Figure VI-20. The three shock regimes in solids .................................................................................84

Figure VI-21. The Seeman-Bohlin camera .........................................................................................86

\section{Section VII-Plasma Physics}

Figure VII-1. Simulation of an interpenetrating plasma .....................................................................90

Figure VII-2. Schematic of colliding plasma experiment ..............................................................99

Figure VII-3. X-ray image of colliding plasma experiment..............................................................90

Figure VII-4. Optical image of a plasma streaming into a magnetic field ....................................91

Figure VII-5. Schematic of regions where laser-plasma instabilities can occur .........................93

Figure VII-6. Wave-matching condition for stimulated Raman scattering .................................93

Figure VII-7. Contour plot of intensity of Raman-scattered light.................................................93

Figure VII-8. Wave-matching condition for stimulated Brillouin scattering ...............................94

Figure VII-9. Diagram of physical mechanism that generates the reflected wave ....................94

Figure VII-10. Spectra for the back-reflected light due to stimulated Brillouin scattering ...... 95

Figure VII-11. Image of laser-induced filamentation ..................................................................96

Figure VII-12. Schematic of the formation of a large-volume hot plasma ..................................98

Figure VII-13. Time history of emission of a gasbag .....................................................................99

Figure VII-14. Schematic of concept of fast-ignitor scheme for heating .....................................101

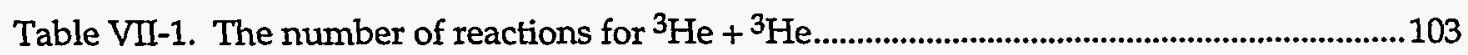

Table VII-2. Selected reactions having rates larger than the ${ }^{3} \mathrm{He}+{ }^{3} \mathrm{He}$ rate..............................103

\section{Section VIII-Radiation Sources}

Figure VIII-1. Total $x$-ray emission, measured as conversion efficiency vs target thickness ..........................................................108

Figure VIII-2. Measurement of transmitted laser energy .................................................................108

Figure VIII-3. Time history of spectrum vs energy in eV.......................................................... 109

Figure VIII-4. Spectrum of the gold M-bands .............................................................................. 110

Figure VIII-5. Temporal history of spectrally integrated emission from the rear side ............110

Figure VIII-6. Spectral character of various elements from $Z=56$ to $Z=63$............................111

Figure VIII-7. Absolutely measured spectra emitted by laser-irradiated targets......................112

Figure VIII-8. Absolute conversion efficiency of laser-irradiated targets...................................113

Figure VIII-9. Effect of angle of incidence of laser on $x$-ray conversion efficiency ................. 113

Figure VIII-10. Equivalent radiation temperature vs time for several gold hohlraums........ 115

Figure VIII-11. Conversion efficiency of various discrete transitions ........................................116

Figure VIII-12. Schematic of double-pulse irradiation technique...............................................118

Figure VIII-13. Schematic of traveling-wave $x$-ray laser ...........................................................119

Figure VIII-14. Schematic of grating technique ......................................................................119

Figure VIII-15. X-ray line emission in 3rd and 4 th order with traveling wave ........................120 
Figure VIII-16. Time history of hard $x$-ray and XUV emission spectra.

Figure VIII-17. Time history of hard $x$-ray (4-2 transitions) and $\mathrm{J}=2-1 \mathrm{x}$-ray laser transition .121

Figure VIII-18. Schematic of experimental setup for plasma imaging.........................................121

Figure VIII-19. X-ray-laser-backlit image of accelerated foil ......................................................... 122

Figure VIII-20. Moiré deflectogram of laser-irradiated plastic target ....................................... 123

Figure VIII-21. Schematic of $x$-ray microscope .......................................................................124

Figure VIII-22. Image of resolution test pattern obtained with $x$-ray laser microscope......... 124

Figure VIII-23. X-ray microscope images of rat sperm nuclei .................................................... 125

Figure VIII-24. Experimental setup for $x$-ray laser interferometry ............................................126

Figure VIII-25. Interferogram obtained using $x$-ray laser interferometer...................................126

Figure VIII-26. Collisionally pumped $x$-ray laser wavelengths ................................................. 129

Figure VIII-27. Streak image of coherent $\mathrm{XUV}$ radiation generated by high-order harmonics.

\section{Section IX-Radiative Properties}

Figure IX-1. Schematic of dot spectroscopy technique.......................................................................135

Figure IX-2. Example of the spectrum of lines of holmium XXXIX .........................................135

Figure IX-3. Ratios of various line emission intensities ................................................................135

Figure IX-4. Schematic of a spectroscopy experiment ................................................................138

Figure IX-5. Temperature measurements from a spectroscopy experiment ...............................139

Figure IX-6. Ratio of the chromium to titanium helium-like 1s2-1s3p intensities ...................140

Figure IX-7. A spectrum of the chromium and titanium produced by irradiating a large bag of gas................................................................................................................140

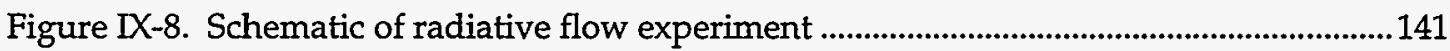

Figure IX-9. Possible experimental packages for radiation flow experiment ............................142

Figure IX-10. Streak-camera record of backlight ...........................................................................142

Figure IX-11. Schematic of setup to study NLTE phenomena ...................................................144

Figure IX-12. Spectrum from absorption of untamped boron nitride ........................................145

Figure IX-13. Experimental results for absorption of a gold backlight ......................................146

Figure IX-14. Schematic of proposed experiment to obtain uniform temperature and density ......................................................147

Figure $\mathrm{DX}-15$. Temperature and density vs time for an overtamped sample .............................148

Figure IX-16. Temperature and density vs time for an untamped sample.................................149

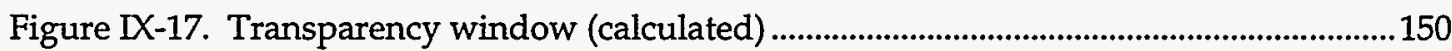

Figure IX-18. Temperature and density parameter configurations for various laser experiments .....................................................................................................151

Figure IX-19. Temperature and density diagram for a specific element (aluminum) .............152

Figure IX-20. Effects of line shapes, line merging, and continuum lowering ...........................154

Figure IX-21. Temperature and density vs time for an undertamped sample...........................158 


\section{Acknowledgments}

The purpose of this document is to present to a general audience the many possible technical areas that can be explored on a high-energy laser. The nature of a document of this kind implies that a certain level of detail will be lost. We accept this, but feel nonetheless that presenting the many areas of past, current and future interest will be informative and will provide a starting point for further discussions.

Since a document of this kind must rely on information from numerous individuals, we would like to make an acknowledgment of these contributions here. The difficult work of scientists from the other institutions in the US should be recognized and we take this opportunity to thank all those working at the high-energy laser facilities in the United States-at the Naval Research Laboratory (NRL), the Laboratory for Laser Energetics (LLE) and Los Alamos National Laboratory (LANL), and the Lawrence Livermore National Laboratory (LLNL) - for the many discussions and contributions that have been made to the generation of much of the work reported. We also would like to acknowledge those that helped directly with the present document, and will do this by scientific area:

\section{Astrophysics and Space Physics}

D. Arnett (U of $A Z$ ), John Castor (LLNL), D. Dearborn (LLNL), M. Horanyi (U of CO), S. Kahn (UCB), L. Klein (Howard), R. Klein (LLNL/UCB), K. Kumar (Howard), E. Liang (Rice), J. Luhmann (UCD), G. Mathews (LLNL), B. Remington (LLNL), D. Savin (UCB), D. Schramm (U of Chicago), T. Weaver (LLNL), J. Weisheit (Rice), S. Woosley (UCSC).

\section{Hydrodynamics}

R. Cauble (LLNL), L. Da Silva (LLNL),

P. Demotakis (Cal Tech), G. Dimonte (LLNL), B. Hammel (LLNL), J. Kilkenny (LLNL), L. Klein (Howard), O. Landen (LLNL), P. Marcus (UCB),
Paul Miller (LLNL), T. Perry (LLNL), T. Peyser (LLNL), B. Remington (LLNL), Ö. Savas (UCB).

\section{Material Properties}

N. Ashcroft (Cornell), R. Cauble (LLNL), R. Christensen (UCD), N. Ghoniem (UCLA), Neil Holmes (LLNL), J. Keady (LANL), M. Kreisler (U of MA), T. Perry (LLNL), J. Wark (Oxford), N. Woolsey (Oxford).

\section{Plasma Physics}

C. Back (LLNL), B. Barletta (LBL), H. Baldis (LULI), R. Berger (LLNL), S. Cramer (UCD), L. Goldman (U of Rochester), A. Kermin (MTT), B. Kruer (LLNL), C. Liu (U of MD), N. Luhmann (UCD), B. MacGowan (LLNL), J. Moody (LLNL), T. Peyser (LLNL), P. Rambo (LLNL).

Radiation Sources

C. Back (LLNL), L. Da Silva (LLNL), R. Freeman (Bell), C. Jacobsen (Stony Brook), M. Key (Rutherford), J. Koch (LLNL), D. Matthews (LLNL), J. Molitoris (LLNL), J. Nilsen (LLNL), M. Richardson (CREOL), D. Umstadter (U of MI).

\section{Radiative Properties}

C. Back (LLNL), L. Collins (LANL), J. Castor (LLNL), U. Feldman (NRL), H. Griem (U of MD), C. Hooper (U of FL), W. Hsing (LANL), S. Kahn (UCB), J. Koch (LLNL), J. Moreno (LLNL), A. Osterheld (LLNL), G. Pollak (LANL), J. Reader (NIST), D. Savin (UCB), J. Seely (NRL), R. Ward (LLNL), J. Weisheit (Rice), B. Young (LLNL).

Facility \& Diagnostics C. Clower (LLNL), G. Tietbohl (LLNL), J. Colvin (LANL), J. Kilkenny (LLNL).

Finally, we would like to thank J. Azevedo, $\mathrm{J}$. Olivera, and S. Jennings for all their help in putting this together. It is by virtue of the hard work invested by all these individuals that this document exists. 
This page intentionally left blank 


\section{Introduction}

There is considerable interest in the general scientific community and in those area of the defense program of the Department of Energy that are concerned with high-energy-density science in scientific studies using high-energy lasers. These communities envision programs of work that are at once scientifically stimulating and accessible to a broadly based constituency. The ideas in this document are the result of long consultation with many individual researchers, from all over the world and from many different fields of science. Their ideas focus on future applications at the National Ignition Facility (NIF).

This document presents both a concise definition of the current capabilities of high energy lasers and a description of capabilities of the NIF. Five scientific areas are discussed (Astrophysics, Hydrodynamics, Material Properties, Plasma Physics; Radiation Sources, and Radiative Properties). In these five areas we project a picture of the future based on investigations that are being carried on today. Even with this very conservative approach we find that the development of new higher energy lasers will make many extremely exciting areas accessible to us.

In the area of astrophysics we find that a high-energy laser can generate such extreme conditions that it becomes possible to study numerous previously inaccessible areas. Among these are the radiative opacity of the outer envelopes of stars, key to our understanding of how stars evolve. Another is the equation of state for stellar material, a major determinant of how dwarf stars behave. There is a close connection between the powerful tools of plasma spectroscopy and the astronomer's inference of conditions in the violent inflowing spiral of material surrounding a neutron star or a black hole that may lie at the center of a quasar.
This very hot material can be recreated in a high-energy-density laboratory experiment. In other experiments we can study a fundamental physical process-mixing at hydrodynamically unstable material interfaces-that is important in many areas of astrophysics, such as in supernova envelopes.

For the future, high-energy laser facilities will furnish excellent opportunities to conduct advanced fluid dynamics experiments. The ability to study fluids has been amply demonstrated with current experiments, and expanded possibilities can be envisioned for the NIF. Because a high-energy laser has the capability to deposit a large quantity of energy in materials over large spatial scales, over long times, and at high energy densities, it will be able to generate hydrodynamic flow conditions more extreme than those obtained with other types of machines. This is especially true with the creation of very high-density, high-temperature volumes of material, which may be used to drive strong shocks and highvelocity flows.

The capabilities of the future high-energy lasers for studying condensed matter physics at extreme conditions will make them the most important new tools in high-pressure physics. For example, we will be able to experimentally validate material behavior for pressures between 1 and $100 \mathrm{TPa}$. The NIF will provide the combination of drive energy and uniformity of drive, with the concomitant large time and length scales for real advances. The NIF will allow us to substantially improve our understanding of dense fluids, pressure ionization, phase boundaries, and energy partition in shocked systems. The basic experimental methods for these studies have existed for some time-the ability to exploit them at ultra-high pressures is still awaiting a NIF-class laser. 
The study of plasma physics has been stimulated over the past four decades by its close connection with the goal of creating fusion as an energy source and with studying aspects of astrophysical plasmas of various types. The advanced capability of the NIF will allow us to produce hot, dense plasmas that are both large and homogeneous, allowing detailed characterization. Thus, for example, we will be able to determine electron and ion temperature, charge state, electron density, and flow velocities of plasmas. This information would allow a wide range of quantitative experiments to be performed. One extremely interesting avenue of research would allow better quantitative measurements on certain nuclear cross sections, and would be made possible by the ability of the NIF to create large volumes of heated plasma in a controlled manner.

In the area of $x$-ray lasers the NIF will allow us to extrapolate existing collisional $x$-ray lasers down to wavelengths of $\sim 20 \AA$. At these wavelengths $x$-ray laser interferometry can be used to measure electron densities in plasmas exceeding solid density. Short-pulse capabilities on the NIF, with pulse lengths in the neighborhood of $100 \mathrm{fs}$, will allow us to develop inner-shell pumped $x$-ray lasers as a viable source. This new class of $x$-ray laser will have the potential to be extended down to as low as $1 \AA$ in wavelength and have a short pulse duration. Further, a NIF-class laser will make possible a wide variety of $x$-ray and particle sources suitable for addressing numerous basic and applied physics questions. The NIF will be capable of producing intense broadband thermal $x$-rays from high- $Z$ radiation converter targets, coherent amplified $x$-rays ( $x$-ray lasers) from high-gain linear plasmas, intense neutron pulses from implosion plasmas, and intense pulses of hard $\mathrm{x}$-rays produced by fast electrons.

The contributions future high-energy lasers can make to science that deals with hot and dense matter will create a watershed for progress in this complex regime so important in our universe. This is true whether the research derives from the fields of astrophysics, material properties, plasma physics, or energy generation.

Richard Lee, Livermore, CA

Richard Petrasso, Cambridge, MA

Roger W. Falcone, Berkeley, CA

January, 1995 


\section{Section I}

\section{High-Energy Lasers in the Context of Current Science}

\author{
Interest in the development of a broad-based \\ scientific community for the use of high-energy \\ lasers can be placed in the wider context of the \\ already existing scientific user communities in \\ the other parts of the world. In the United \\ Kingdom, France, Germany, and Japan, there \\ exist high-energy lasers, supporting diverse \\ programs of work based, to various degrees, on \\ scientific user communities. For a combination \\ of reasons, the development of the same diverse \\ scientific user base has not occurred to any large \\ degree in the USA, although we have had \\ world-class high-energy lasers since the \\ inception of these facilities in the early 1970s. \\ First and foremost is the inescapable fact \\ that the Inertial Confinement Fusion (ICF) \\ program in the USA has had a substantial \\ classified component, and this has created an \\ impediment to the free transmission of \\ information. Although there have been \\ unclassified components of the ICF program in \\ the USA, the residual effect of classification was \\ to stunt growth in the scientific community. As \\ mentioned above, this must be seen against a \\ worldwide background where scientific-based \\ communities have flourished. Thus, a lack of \\ development here cannot easily be explained as \\ being due to a lack of relevance of the high- \\ energy laser to basic or applied science or its \\ incompatibility with scientific investigations. \\ Recent changes have modified the \\ prevailing atmosphere and will, hopefully, be \\ beneficial to the development of a scientific user \\ community. The first of these changes is very \\ recent (December of 1993), with the \\ declassification of the concept of indirect-drive \\ (i.e., radiation-drive) ICF, which was the \\ dominant program. This declassification is a
}

result of a number of politico-economic adjustments, including the easing of the EastWest tension and a commitment from the Department of Energy (DOE) to make a transition into this less-than-Cold-War era. Second, there is the active pursuit of a "dualuse" strategy, which in the present case can simply be seen as a concerted effort by the $\mathrm{DOE}$ and other government agencies to have their capabilities utilized more broadly. Third, important changes are now taking place in the ICF program in response to this new climate. There is now a commitment to scientific applications of high-energy lasers. These applications set the stage for this document.

The changes that have been occurring in the ICF program, the DOE, and in the USA generally, only lay the foundation for this quest for scientific participation; successful development of a user community is not guaranteed by our commitment alone. This document represents an attempt to catalog the many possible uses for a high-energy laser facility. Therefore, this effort is in support of the broad concept of the development of a user community, which has been addressed by the organization of workshops and extended individual discussions.

The workshops and discussions have produced a number of intriguing possible applications, which will be found below. The method used to evaluate the many proposed topics of interest was a straightforward analysis of the applicability of the suggested research to the high-energy laser facilities of central importance here. We have relied extensively on the many contacts mentioned above. 
This page intentionally left blank 


\section{Section II}

\section{Overview of Science on High-Energy Lasers}

\section{A. Astrophysics and Space Physics}

The reason for considering experiments on high-energy-density plasmas in connection with astrophysics and space physics is simply that such plasmas are a dominant aspect of many astronomical objects, whether in our solar system, in other stars, or in other galaxies. The astrophysicist or space physicist is faced with the problem of deciphering the few clues the observations provide in order to learn about the objects' nature. The clues most often consist of plasma radiation, and a full familiarity with what plasmas actually radiate is needed to learn about the astronomical objects. In other cases the theoretical astrophysicist or space physicist builds up a complete conceptual structure of the object, and then tests aspects of the structure against the observations. In this case the dynamics of plasmas form one of the basic building blocks of the theory, but these dynamics require observational support themselves.

The goal of astrophysically motivated laboratory experiments is to create in the laboratory a sample plasma similar to what would be found in the astronomical body, then to study its properties-its radiation or its thermodynamic state, whichever is critical in the astrophysical picture. The properties that are studied will be found under the other project headings of this report: Hydrodynamics, Material Properties, Radiative Properties, and so on. Astrophysics does not, in general, provide new plasma properties that need to be studied, but rather gives a specific motivation for studying certain of the ones already known.

- The discussion in Section IV will highlight a few instances in which there is a specific astrophysical motivation for studying a particular plasma. Among these is the radiative opacity of the outer envelopes of stars, key to our understanding of how stars evolve. Another is the equation of state of stellar material, which is a major determinant of the behavior of dwarf stars-the white dwarfs left over at the end of stellar evolution and the brown dwarfs that are the failed almost-stars.

There is a close connection between the powerful tools of plasma spectroscopy and the astronomer's inference of conditions in the violent inflowing spiral of material surrounding a neutron star or a black hole that may lie at the center of a quasar. This very hot material can be re-created in a high-energy-density laboratory experiment. In other experiments we can study a fundamental physical process-mixing at hydrodynamically unstable material interfacesthat is important in many places in astrophysics, such as in supernova envelopes, and in terrestrial applications as well.

Astronomy provides some of the driving interest in these and other laboratory experiments, and it also provides another laboratory and an additional data set. Merging the data from laboratory experiments with astronomical observation will improve our knowledge of the universe, as well as our understanding of the behavior of matter found here on earth. (See Section IV for a full discussion of astrophysics and space physics on high-energy lasers.) 


\section{B. Hydrodynamics}

Interest up to the present in performing hydrodynamic experiments can be divided into two broad areas. First, there is the interest in stable hydrodynamics. This includes the study of pressures obtainable, shock characteristics, and other aspects of the hydrodynamics problem as these affect various aspects of, for example, ICF. It includes looking at how these experiments permit testing of hydrodynamics simulation capabilities.

Secondly, there is a keen interest in unstable hydrodynamic flow. This interest includes understanding flow in the linear growth regimes, through the nonlinear regimes, to turbulent flow. The instabilities that have been tackled include Rayleigh-Taylor, Richtmyer-Meshkov, and Kelvin-Helmholtz. These experiments have attacked the unstable flow problem in a highenergy-density regime, and that in itself is a unique aspect of the work.

In addition, the dimensionality of the flow studied can be changed in the experiments. In preliminary experiments, such as those illustrated in Section $V$, the desire is for simplicity, but this is a goal and is not always achieved. On the other hand, there are experiments in converging spherical geometry, such as those required for ICF, as contrasted with more idealized planar geometry experiments performed for basic information. Examples of both stable and unstable hydrodynamics in spherical and planar geometry are shown in Section V.

For the future, high-energy, laser-based facilities can furnish excellent opportunities to conduct advanced fluid dynamics experiments. This has been amply demonstrated with current experiments illustrated here, and expanded possibilities for further investigations can be envisioned for the NIF. The capability of a laser to deposit a large quantity of energy in materials over large spatial scales, over long times, and at high energy-densities means that it will be able to generate hydrodynamic flow conditions more extreme than those obtained with the more familiar machines such as wind tunnels or shock tubes. This is especially true concerning the creation of very high-density, high-temperature volumes of material, which may be used to drive strong shocks and high-velocity flows.

The extensive set of diagnostics available to study laser-produced flows facilitates measurements of the quantities of interest, including interfacial geometries, velocities, densities, and /or temperatures. The NIF will be of great interest for the experimental study of the physics of high Mach number flow and the instabilities associated with the presence of certain material conditions and interfaces. Typically, large Mach numbers have been difficult to attain in the laboratory, because achieving the high flow velocities requires a very high-enthalpy source, such as very hightemperature, high-pressure gas, which is problematic. The NIF, however, should excel in producing a high-enthalpy source (i.e., a hohlraum). Using the hohlraum as the source, the NIF will be able to generate very strong shocks and large Mach number flows.

This possibility of testing conventional theory under extreme conditions opens up a large perspective toward the understanding of various astrophysical phenomena involving the propagation of blast waves and the plasma flow such as those generated by supernova explosions. Compressibility of the materials studied and its relationship to the onset of turbulence is also an important connection to be probed under the unprecedented conditions attainable with these high-energy-density sources. Finally, because of the larger spatial scales, longer times, and higher energies provided by the NIF, it will permit fluid dynamics investigations to be

- Larger, thereby increasing relative spatial resolution.

- Longer, thereby allowing more growth time for the development of features.

- More energetic, thereby helping to maintain drive conditions and reduce decompression effects.

Thus, it will permit the detailed study of phenomena that to the present have been studied experimentally exclusively by astronomical observation, or only by computer theoretical simulation. (See Section V for a full discussion of hydrodynamics on high-energy lasers.) 


\section{Material Properties}

The interest in material properties at extreme conditions has developed from both fundamental and applied interest. For, example, the challenges of understanding the equation of state (EOS) at extreme conditions requires the development of a model that can treat strongly coupled systems, with all the difficulties of hot, dense matter. On the other hand, we know that ICF and astrophysical studies require basic EOS data in high-energy-density regimes. The evolution of the study of material properties has come to include, not only EOS studies performed in both directly and indirectly driven targets, but opacity measurements, and strength of materials studies.

The capabilities of the NIF for studies of condensed matter physics at extreme conditions of pressure and temperature will make it the most important new tool in high-pressure physics. While we will describe in the following sections vital contributions that only the NIF can make to this field, we want to make it clear that studies in this field will make an essential contribution to other fields as well. A broad and precise knowledge of condensed matter properties for pressures between 1 and $100 \mathrm{TPa}$ (between 10 and $1000 \mathrm{Mbar}$ ) and temperatures up to a few hundred electron volts will be essential for the design, simulation, and interpretation of experiments for other scientific applications of the NIF (such as hydrodynamics, planetary physics and astrophysics, and radiative properties) as well as for the ICF program. For example, we have yet to understand the behavior of hydrodynamic instabilities in the pressure range for which pressure ionization becomes important. We must have a "caloric" equation of state to interpret the entropy fluctuations and to determine the appropriate invariants for highMach-number flows under these conditions.

Presently, no models of material behavior for pressures between 1 and $100 \mathrm{TPa}$ have been experimentally validated. Impact and static experiments provide a wealth of data at low pressures, below a few Mbar, and statistical mechanical models such as Thomas-Fermi theories are believed to be valid at very high pressures. The upper bound is approximate, and increases with $\mathrm{Z}$.

At tens of TPa (hundreds of Mbar), the models that do exist only agree within about $30 \%$ in density, and vary by as much as a factor of two in temperature along a shock adiabat (Hugoniot). These models differ quantitatively and in functional form, and the discrepancies between the models are functions of density, pressure, and temperature. Although a few shock-wave experiments have been carried out in this pressure range in the former Soviet Union (driven by a nuclear device), none have been sufficiently accurate to test theory. Over the last decade, a few laser-driven experiments have probed this region of pressure with even less accuracy.

The NIF will provide the combination of drive energy, uniformity of drive, and the concomitant large time and length scales for real advances. This effort will substantially improve our understanding of dense fluids, pressure ionization, phase boundaries (such as in proposed plasma phase transitions), and energy partition in shocked systems. The basic experimental methods for these studies have existed for some time- the ability to exploit them at ultrahigh pressures is still awaiting a NIFclass laser.

We need to know material properties over wide ranges of density and pressure. Most previous experiments have been shock-wave experiments on the principal Hugoniot. The pulse-shaping capabilities of the NIF are essential to reach off-Hugoniot states, especially near the isentrope. Cold compression, along the roomtemperature isentrope, will be limited by the eventual insulator-metal transition in diamond, which will occur at about $1 \mathrm{TPa}$.

While experiments and theory have emphasized shock properties in the past, these are merely the simplest and most fundamental measurements. There is yet to be any experimental data on transport properties at these extreme conditions, such as thermal and electrical conductivity, and we note that the hydrodynamics itself will depend on radiative transport away from the shock front at the highest pressures of interest. 
Finally, we note that for the first time an intense source of neutrons will be available from the ignition process. This will allow us to heat along isochores, or paths of constant density, to tens of electron volts in many metals, allowing access to a region of state space previously unreachable in any experimental environment. (See Section VI for a full discussion of material properties on high-energy lasers.)

\section{Plasma Physics}

The study of plasma physics has been stimulated over the past four decades by its close connection with the goal of the creating fusion as an energy source and with astrophysical plasmas of various types. That is, the luminous matter in the universe is composed almost entirely of plasma, and this makes the scientific investigation of plasma of the utmost importance. Furthermore, the NIF will allow us to make wide-ranging and detailed studies of universal plasma conditions, an opportunity, to the best of our knowledge, that will not be afforded on any other facility.

The rather large number of experiments that high-energy lasers permit in the area of plasma physics indicates the close connection among many of the applications. A number of the interesting phenomena that take place in plasmas (such as parametric instabilities) rely on a large degree of homogeneity in plasmas that are both hot and dense. Most plasma physics experiments to date have been complicated by the large gradients and small scale of the plasmas produced by current lasers.

The advanced capability of the NIF will allow us to produce hot, dense plasmas that are both large and homogeneous, allowing detailed characterization. Thus, for example, it will be possible to determine electron and ion temperature, charge state, electron density, and plasma flow velocities. This in turn would provide a medium in which a wide range of quantitative experiments can be performed.

Some of the laser-plasma interaction experiments that will be possible on the NIF will be developments of those already attempted on smaller, less well-characterized plasmas.
However, it will be possible to go beyond such experiments, which were geared to the needs of the current ICF program, and investigate a wide range of scientifically important interactions.

There is general interest in having an advanced high-energy laser facility, which should include a beamline for short-pulse (i.e., pulse durations less than $1 \mathrm{ps}$ ) high-power experiments. This capability is especially important for many basic plasma physics studies. These include

- Relativistic, ultrahigh-intensity regimes of laser-matter interaction

- Ponderomotive effects

- Relativistic self-focusing and filamentation

- Laser beam channeling

- Intense harmonics generation

- Ultrahigh B-field generation with ancillary studies of the resultant physical processes

- Strongly driven instability regimes

- Generation and transport of high electron fluxes in a plasma

- High-gradient accelerator schemes

- New x-ray lasers

- High temporal resolution diagnostics Many of these will be discussed below.

Another new area of focus will be the study of magnetic fields, as large as $100 \mathrm{MG}$, which are generated by temperature and density gradients present in specially produced plasmas. Faraday rotation of an optical probe beam and/or Zeeman splitting of spectral lines should make it possible to investigate and quantify these magnetic fields. The generation of large currents of fast electrons, produced by SRS and Two-Plasmon decay in plasmas at 0.25 of the critical electron density, will be a related area of study. Coupling these electrons into a high- $Z$ metal converter should allow the production of short-duration bursts of 50 - to $100-\mathrm{kV} x$-rays with numerous potential radiographic applications. Further, the feasibility of performing nuclear reaction experiments in the large, hot, well-characterized plasmas that will be produced on the NIF has been demonstrated in smaller experiments at the Nova laser facility.

In general, these larger plasmas will translate into longer interaction lengths, greater 
homogeneity, higher temperatures, longer time scales, and reduced velocity gradients. Therefore, the NIF laser will make strong contributions to fundamental plasma physics. (See Section VII for a full discussion of plasma physics on highenergy lasers.)

\section{E. Radiation Sources}

The wavelength of current lasers occurs in approximately the visible wavelengths. The conversion of this visible wavelength into other (usually shorter) wavelength radiation has been one of the dominant themes of high-energy laser experiments. This generation of radiation has many practical applications beyond the intrinsic theoretical interest in production mechanisms, as absorption sources, $x$-ray heating sources, $x$-ray lasers, etc.

The development of these light-source capabilities will be broken down into three generic areas. First, there is the effort to use the high-energy laser to make spectrally continuous or quasi-continuous sources for use as absorption sources and as heating sources for the application of radiatively heating matter. Second is the work on the conversion of the high-energy laser into narrow-band radiation sources. Here the effort is usually quantified by the conversion efficiency into a single-line transition or into a small, closely grouped set of lines. Finally, there is the effort to further develop the $x$-ray laser, which work, of course, overlaps with the applications of $\mathrm{x}$-ray lasers.

A NIF-class laser will make possible a wide variety of $\mathrm{x}$-ray and particle sources suitable for addressing numerous basic and applied physics questions. The NIF will be capable of producing intense broadband thermal $x$-rays from high- $Z$ radiation converter targets, coherent amplified $x$-rays ( $x$-ray lasers) from high-gain linear plasmas, intense neutron pulses from implosion plasmas, and intense pulses of hard $x$-rays produced by fast electrons. Accurate energy spectra and absolute measurements of the conversion of laser energy into all types of radiation and particle fluxes will play an important role in benchmarking our basic understanding of laser plasma interactions and atomic physics.

Broadband $\mathrm{x}$-rays generated from laserproduced plasmas can be used to produce and characterize large and uniform plasmas relevant to ICF and astrophysics. Previous experiments using this technique have yielded important results which can be extended to high temperatures and densities. The high temperatures and densities produced during implosion and subsequent ignition will be an unmatched source of continuum $x$-rays extending from the $\mathrm{XUV}$ to $\mathrm{MeV}$ with pulse durations less than $100 \mathrm{ps}$.

$\mathrm{X}$-ray lasers, besides being important coherent radiation sources, offer a critical test of our atomic modeling. On the NIF, $x$-ray lasers will allow us to extrapolate existing neon-like and nickel-like collisional $x$-ray lasers down to wavelengths of $\sim 20 \AA$. At these wavelengths $x$-ray laser interferometry can be used to measure electron densities in plasmas exceeding solid densities. Short-pulse capabilities on the NIF, with pulse lengths $\sim 100 \mathrm{fs}$, will allow us to develop inner-shell pumped $x$-ray lasers as a viable source. This new class of $x$-ray laser will have the potential to extend down to $1 \AA$ in wavelength and have a short pulse duration. High-order harmonic generation, an alternative source of coherent radiation, can be used to produce tunable coherent $X U V$ radiation.

Neutron production will exceed $10^{18}$ in single 100-ps pulses, making the NIF a potentially useful source for producing uniform high-density and low-temperature plasmas (i.e., strongly coupled plasmas). Fast electrons with hundreds of $\mathrm{keV}$ in energy will be generated by parametric instabilities, providing another potential source of high-energy $x$-rays for plasma backlighting and probing.

These radiation sources are supplemented by the possibility of using radiation enclosures, or hohlraums, for the generation of radiation environments and $x$-ray drive fluxes. Hohlraums will be able to produce far in excess of the $200-\mathrm{eV}$ equivalent radiation temperature sources now available on the current high-energy laser facilities. These sources will not only be of higher effective temperature but will also be able to 
provide uniform $x$-ray drive over far larger areas than can now be imagined on present-day systems. Thus, the advantages of using $x$-ray heating for the study of hot, dense matter will be greatly enhanced with the advent of the NIF. (See Section VIII for a full discussion of radiation sources on high-energy lasers.)

\section{F. Radiative Properties}

The importance of the study of radiative properties in high-energy-density plasmas derives from three separate factors. The first is that the radiative property can be the best indicator of the level of scientific knowledge in a particular area. So, for example, when one is interested in the development of new descriptions of atomic structure, the most important measurements to be made are the accurate determination of transition energies. Moreover, as with the case of spectral line broadening, one can develop sophisticated plasma theoretical constructions, kinetic theories, or scattering theories and find that the simplest place to test the theory is through a prediction of a line shape, which can then be measured.

Second, radiative properties provide the classic example of the non-interfering probe. Thus one can obtain fundamental information on the plasma by looking directly at the emission or the absorption spectrum. Spectral formation in its most sophisticated forms can provide information on the plasma ionization balance, the rate processes, the densities, the temperatures and the fluctuation levels. The radiative properties are therefore a powerful diagnostic of the plasma state.

Third, although radiative properties provide an important test bed for theoretical development and provide diagnostic information, they are also necessary as primary data for numerous other studies. For example, spectral line lists are inadequate for many of the charge states of heavier elements. Therefore, the categorization of the energies of highly ionized species becomes an end in itself, as data tables are necessary for the myriad uses to which spectra are put. In this way, line shapes, line widths, and line shifts must be measured for use in radiation-hydrodynamic simulations of entities as small as an ICF microsphere implosion and as large as a galaxy.

These factors together explain the interest in radiative properties of hot, dense matter. It is clear that the largest part of the community of scientists outside the $x$-ray laser area that are involved in high-energy laser experiments are concerned with measuring radiative properties. It is a fertile area with much to be gained from the existing high-energy laser facilities and even more to be gained with the advent of the NIF.

Some of the possible applications for experiments on high-energy lasers are presented in the Section on Radiative Properties; here we have only broadly summarized a few of the ideas to provide an indication of the type of experiments being considered. (See Section IX for a full discussion of radiative properties to be derived from high-energy laser experiments.) 


\section{Section III}

\section{Experimental Capabilities}

Before illustrating the experimental possibilities of high-energy lasers, we will discuss the definition of a high-energy laser facility.

We note that the repetition rate of the experiments is relatively low when compared to tabletop laboratory experimental facilities. On the other hand, the system has, for a high-energydensity production facility, a fairly rapid turnaround time. The inherent problem in using a facility to obtain both high temperatures and high densities is that it tends to cause various high levels of destruction, which increases the time it takes to ready the facility for a new shot. For this reason, the high-energy laser has a relatively low duty cycle (i.e., one shot per hour). However, even when compared to the duty cycle of tabletop facilities (i.e., greater than one shot per hour), the high-energy lasers provide benefits that can help offset their comparatively low duty cycle.

The most obvious factor in support of the high-energy laser for research work, in spite of the relatively low duty cycle, is the fact that the diagnostics complement that can be brought to bear on a single experiment is very large. Second, as a result of the diagnostics and the properties of lasers, is the fact that the high-energy lasers can perform experiments that cannot be performed at any other type of facility. Thus, the high-energy laser can be seen as a distinct breed of facility that permits novel regimes to be investigated, but which allows these investigations to occur in real time.

\section{A. Definition of Existing Facility}

The following definition of an existing highenergy laser facility is based on the Nova laser facility at LLNL. Although this is currently the largest facility, it is generically similar to the other facilities currently extant. Note that the definition of the NIF laser, which is contained below in Subsection B), is being developed to a large degree with the assistance of the LLNL Laser Science program, and most of the facility information has been obtained from individuals associated with that program.

\section{The Laser}

\section{Laser Beams}

The Nova facility has ten separate beam lines, all of which are roughly equivalent. The laser is based on the master oscillator power amplifier (MOPA) Nd:YLF configuration, where the initial seed laser is generated in a desired temporally shaped pulse. The amplification occurs in a long chain of amplifiers separated by spatial filtering pinholes in increasingly larger diameter sections. The $1-\omega_{0}(1.053-\mu \mathrm{m})$ wavelength beam arrives at the target chamber with approximately 10 kilojoules of energy in a beam diameter of $74 \mathrm{~cm}$. This assumes a pulse that is temporally a 1-nssquare pulse-that is, the pulse rises in 100 ps to a uniform intensity and persists for $1 \mathrm{~ns}$ until it falls in $100 \mathrm{ps}$. The frequency of each beam is then either doubled (using a KDP crystal) to a $2-\omega_{0}$ (green) beam, or tripled to a $3-\omega_{0}$ (blue) beam, for final focusing down into the target chamber. The conversion efficiency of the doubling process is approximately $60 \%$, yielding $\sim 4000$ joules of energy in the beam to be focused into the target chamber. The ten beams are balanced to produce $\leq 5 \% \mathrm{rms}$ deviation in energy.

The 1- $\omega_{0}$ beam is of high spatial uniformity; however, the nonlinearity of the up conversion to 
$2 \omega_{0}$ or $3 \omega_{0}$ amplifies any amplitude modulation in the beam. Several techniques can be used to smooth the beam, a major consideration for directly irradiating a surface where one desires uniformity. First, a random phase plate can be used to introduce a randomness in the phases of the beam. This creates an effectively smoother intensity distribution by overlapping many small beamlets of random phase created from the largeaperture beam. Second, the beam can be split up into several pieces using wedges that allow an effectively flat intensity distribution over the focal spot. Third, it is possible to introduce a bandwidth on the normally monochromatic beam; when combined with a diffraction grating, this will be used to move the focal spot, resulting in a temporally smoother intensity.

The limiting factor in the firing of the beam is the cooling-down time for the large disk amplifiers. This limits a particular laser beam line from firing more than once every hour. However, the complexity of the diagnostic setup and alignment of the target can be, and often is, the limiting factor in the turnaround time of the experiments.

\section{Beam Pointing, Aiming, and Synchronicity}

As the beam enters the target chamber, it is focused into the center using an $f / 4.3$ lens that will produce a minimum spot size of $150 \mu \mathrm{m}$ in diameter. The other option at the present time for the beam is the insertion of $f / 8$ lenses. This is a rather new option.

The accuracy of the focusing, or pointing, is $\pm 30 \mu \mathrm{m} \mathrm{rms}$, with a maximum displacement of the beam centerline from target chamber center of $2 \mathrm{~cm}$. The beams can be sent to three different target areas: a one-beam chamber, a two-beam chamber with the beams coming in opposing ports, and a ten-beam chamber. In the ten-beam chamber the beams come into the chamber in two clusters of five beams, with the clusters entering on opposite sides of the midplane. The five beamlines in a cluster are placed in a five-sided symmetry, and come into the chamber in a cone with a $50^{\circ}$ half angle. The synchronicity is $\pm 20 \mathrm{ps}$ at target chamber center.

\section{Laser Pulse Shapes}

Pulse durations can vary from 100 - to 600 -ps Gaussian pulses to 1- to 5-ns-square pulses. There is also flexible pulse-shaping capability, so that the energy can be delivered in 2- to 3-ns shaped pulses with intensity contrast between peak and foot of the pulse greater than ten. Two of the beams can be delayed, independently of each other, by up to $25 \mathrm{~ns}$ after the main beam. This last capability has been found useful for providing a source of backlighter $\mathrm{x}$-rays.

\section{Laser Shot Rate}

The laser currently fires at a rate of approximately 115 shots per month. This is consistent with the fact that the laser fires about seven shots per day utilizing a two-shift system. The week has four scheduled experimental days and one day maintenance.

\section{Beam Diagnostics}

There are several diagnostics of beam performance available. First, the pulse shape is monitored in the master oscillator for temporal signature and energy. Next, the propagated beam can be monitored for the total 1- $\omega_{0}$ energy and the temporal variation of the 1- $\omega_{0}$ energy. Further, images can be made of the near and far field of the 1- $\omega_{0}$ beam.

The up-converted beam, in either $2 \omega_{0}$ or $3 \omega_{0}$, can be monitored in the chamber for absolute intensity as well as temporal intensity, and images of the beam front can be produced in the far or near field.

\section{Diagnostic Capabilities}

In contrast to smaller facilities, the diagnostics on Nova are engineered to be routinely run on any shot by facility staff. Making this possible involved applying professional engineering standards to the diagnostics. This has avoided the more usual operating procedure of several Ph.D.-level scientists working for many weeks to get their principal diagnostic installed for their series of experiments, and after an intense campaign of experiments removing their diagnostic. 
In the long run, this is an efficient use of resources. Also it is necessary for efficient facility scheduling, as the user will run in the mode of doing different sets of experiments in any one week. This mode of scheduling precludes the exclusive use of a target chamber for blocks of a week or more, but has the advantage of giving experimentalists time to analyze a set of data before proceeding with an experimental series.

To achieve a high standard of engineering for the diagnostics, experimentalists work with engineers and technicians for many months to design, build, and implement the diagnostics, with the goal of implementing diagnostics that can be routinely run by facility staff for any user.

The chamber can be quite large-for Nova it is $2.4 \mathrm{~m}$ in radius. However, the chamber will pump to vacuum in less than one hour. It is not routinely vented during the four- or five-day shooting week. For shots where the target geometry does not change, this allows shots to be fired without realignment of the laser beams. As a result, diagnostics are designed to be operated without venting the target chamber.

For flexibility, all of the $x$-ray imagers and $x$-ray spectrometers that operate close to the target have been made to be interchanged using one of six standardized vacuum-load lock and manipulator devices, known as SIMs (for six-inch manipulators). These manipulators provide a standard for several facilities. In excess of twenty diagnostics are now mounted on the carts that fit into the SIMs. This standardization allows key diagnostics to be moved from one location on the target chamber to another location over a period of about 1 hour, so that the configuration of target diagnostics can be rapidly changed for different experimental campaigns. Out of the 30 or so shots that are fired most weeks, diagnostics are typically moved every $2-4$ shots, the number of consecutive shots that are fired in any one campaign.

\section{Overview of Target Chamber Diagnostics}

Table III- 1 lists the diagnostics available on Nova. The list includes the dozen different $x$-ray imaging diagnostics, $x$-ray spectrometers, neutron diagnostics, and optical diagnostics.

The $x$-ray imagers are reflective grazing incidence $x$-ray microscopes, pinholes or coded apertures, or point-projection systems. Because it is important to have time resolution, the detectors for the imagers are $x$-ray streak cameras, gated microchannel plate (MCP) detectors, or simply $x$-ray film with flash backlighting.

The x-ray spectrometers available are crystal spectrometers (Bragg or Laue), broad-band diode arrays, or grazing incidence grating spectrometers. Again, the detectors are x-ray streak cameras, gated MCP detectors, or x-ray film.

The neutron diagnostics are yield (by activation), high- and low-resolution energy spectra, emission-time diagnostics, and neutron imaging.

The optical diagnostics are streaked optical spectrometers, used mainly for stimulated Brillouin and Raman scattering in either one location or in an array of different angular locations; optical and UV imagers that either go onto streak cameras or gated optical imagers; and arrays of calorimeter modules.

Note that in Table III-1 we have listed the abbreviated names of the diagnostics, as these abbreviations are used in many of the figures. The various uses of the diagnostics have been covered in the section above.

\section{$X$-ray Imagers}

Nova has had for several years reflective grazing incidence $x$-ray microscopes, two of the Kirkpatrick-Baez design and one of the Wolther design. The detectors for one of the four-channel Kirkpatrick-Baez x-ray microscopes are film. The other microscope has four time-gated $x$-ray detectors using gated MCP (microchannel-plate) technology. 1

The Wolther microscope has a spatial resolution of approximately eight microns, although in one direction there is a large scatter caused by the surface roughness of the $x$-ray optic. This instrument has been widely used on Nova. ${ }^{2}$ The collecting solid angle of the optic is large enough that only a small sector of the annular imaging region need be used to form an image. Five separate $5^{\circ}$ sectors of the annular optic are used to make it into a five-channel system, with an x-ray streak camera on one channel and gated MCP detectors on each of the other four channels. ${ }^{3}$ This instrument has been widely used on $x$-ray backlighting experiments 
Table III-1. Diagnostics available on Nova, with their abbreviated names. These include x-ray imagers and spectrometers, neutron diagnostics, and optical imagers and spectrometers.

\begin{tabular}{|c|c|c|c|c|c|}
\hline Name & Abbrev. & & Name & Abbrev. & \\
\hline$\underline{X \text {-ray imagers }}$ & & & $\underline{X \text {-ray spectrometers }}$ & & \\
\hline Wolther $x$-ray microscope & $22 X$ & 1 & Streaked crystal spectrometers & NSCS, & 2 \\
\hline Gated x-ray pinhole camera & GXI, WAX, & 4 & & Keanetech & \\
\hline Gated soft $x$-ray framing camera & $\begin{array}{l}\text { GACS, GSIX } \\
\text { SXRFC }\end{array}$ & 1 & $\begin{array}{l}\text { High-resolution streaked } \\
\text { spectrometer }\end{array}$ & HICKS & 1 \\
\hline Streaked soft $x$-ray imager & NSDSS & 1 & Static crystal spectrometers & Henway, & 6 \\
\hline Ring-aperture microscope & RAM & 1 & & PUS & 1 \\
\hline Streaked slit/array imager & SSC/SMP & 2 & High-resolution crystal spectrometer & HOPS & 1 \\
\hline Kirkpatrick-Baez microscopes & $8 x$ & 2 & Gated crystal spectrometer & $\begin{array}{l}\text { TOPS } \\
\text { IXuVs }\end{array}$ & 1 \\
\hline Axial pinhole cameras & APH & 2 & $\begin{array}{l}\text { Gated imaging XUV spectrometer } \\
\text { Laue spectrometer }\end{array}$ & $\begin{array}{l}\text { IXUVS } \\
\text { HETS }\end{array}$ & 1 \\
\hline Large-area backlighting & & & Low-resolution $x$-ray diode array & Dante & 2 \\
\hline $\begin{array}{l}\text { Point-projection spectroscopy } \\
\text { Soft x-ray microscope }\end{array}$ & PPS & $\begin{array}{l}2 \\
1\end{array}$ & $\begin{array}{l}\text { Low-resolution high-energy } \\
\text { fluorescers }\end{array}$ & FFLEX & 2 \\
\hline XRL beam divergence camera & Cube & 1 & Spatial coherence diagnostic & & 1 \\
\hline \multirow[t]{4}{*}{ XRL spatial-coherence diagnostic } & & 1 & Grazing-incidence spectrometer & COFFIN & 1 \\
\hline & & & High-resolution spectrometer & HIRES & 1 \\
\hline & & & $\begin{array}{l}\text { Time-resolved soft } x \text {-ray } \\
\text { spectrometer }\end{array}$ & SFFD & 1 \\
\hline & & & $\begin{array}{l}\text { Gated grazing-incidence } \\
\text { spectrometer }\end{array}$ & McPIGS & 1 \\
\hline Neutron diagnostics & & & \multicolumn{3}{|l|}{ Optical spectrometers and imagers } \\
\hline Yield & $\mathrm{Cu}$, In scin & 3 & Streaked/gated imager & SOP & 2 \\
\hline Bang time & $\begin{array}{l}\text { NETMCP, } \\
\text { GaAs }\end{array}$ & 3 & Streaked optical spectrometers & SOS, BSS & 4 \\
\hline Burnwidth & NTD \& GaAs & $?$ & Multiple streaked spectroscopy & MATRES & 1 \\
\hline High-resolution high-sensitivity & LaNSA & $\begin{array}{l}2 \\
1\end{array}$ & $\begin{array}{l}\text { Spatially discr. streaked optical } \\
\text { spectroscopy }\end{array}$ & SDOSS & 1 \\
\hline & & & Calorimeter array & EBM & 1 \\
\hline $\begin{array}{l}\text { Medium-resolution neutron } \\
\text { spectrometer }\end{array}$ & NTOF & 3 & Full-beam backscatter & sos5 & 1 \\
\hline Ultrahigh-resolution spectrometer & fNTOF & 1 & & & \\
\hline $\begin{array}{l}\text { High-resolution high-sensitivity } \\
\text { spectroscopy }\end{array}$ & LANL Ti & 1 & & & \\
\hline Neutron imager & NPAM & & & & \\
\hline
\end{tabular}

on Nova where accuracy, ease of alignment, and sensitivity are important. Note that the solid angle/channel of the Wolther microscope is $3 \times 10^{-6} \mathrm{sr}$ compared to about $10^{-7} \mathrm{sr}$ for a Kirkpatrick-Baez microscope.

For higher $x$-ray energy imaging, simpler systems are also being used on Nova. Crucial information on symmetry and the mix of pusher material into a fuel is obtained from imaging implosion cores at higher photon energy and better spatial resolution. The higher energy implies high sensitivity, and the requirement of good spatial resolution implies good time resolution to freeze plasma motion.

To tackle this problem, gated pinhole and coded aperture systems have been used. 
To ensure adequate sensitivity, the 5- to $10-\mu \mathrm{m}$ pinholes of the instruments are brought as close as $5 \mathrm{~cm}$ from the target. Because the instruments are used to record signals from $\geq 3 \mathrm{keV}$, relatively robust $x$-ray filters can be used, allowing the pinholes to survive for a few shots the target shrapnel from the $20-40 \mathrm{~kJ}$ on target.

Time resolution is achieved by gating 0.5-mm-thick coated MCPs. At present, several coating configurations are routinely used: a four-channel open-ended strip configuration, a 16-channel serpentine arrangement, and 16 channels onto four parallel strips. The motional blurring is given by

$$
\delta \mathrm{x}=\mathrm{v} \delta \mathrm{t}
$$

and so the 80-ps gate time that is easily achieved is adequate for $10-\mu \mathrm{m}$ resolution with velocities of approximately $10^{7} \mathrm{~cm} / \mathrm{s}$. For improved resolution, 30-40-ps gate times are becoming available with thinner MCPs.

$X$-ray backlighting has been in use for laserproduced plasmas for several years, but for implosions and in-flight measurements of higherdensity planar foils, higher-energy backlighting is required. Several developments have made higher-energy $(\sim 5-7 \mathrm{keV})$ streaked $x$-ray backlighting possible. The most important are:

- Random phase plates are now used on the backlighting beams to produce a smooth, virtually monochromatic source of $x$-rays by irradiating, for example, an iron disc at $\sim 10^{15} \mathrm{~W} / \mathrm{cm}^{2}$.

- A slit imager onto a close-in x-ray streak camera with the photo cathode about $50 \mathrm{~cm}$ from the plasma allows for adequate sensitivity for recording this backlighting source.

For soft $x$-ray studies, two gated and streaked soft $x$-ray imaging instruments are in use. A schematic of the gated soft $x$-ray framing camera is shown in Fig. VI-10 (see Section V, Hydrodynamics). For two soft channels, a combination of grazing incidence mirrors and filters defines two bands of soft $x$-rays. The third and highest energy channel has its passband defined by a filter. Images in $\mathrm{x}$-ray bands at $500 \mathrm{eV}, 1 \mathrm{keV}$ and $2.5-3 \mathrm{keV}$ are recorded with 100-ps gate time at four different times, giving twelve images in total. This instrument allows the spatial evolution of the soft $x$-ray emitting region of a laser plasma to be accurately measured.

A major diagnostic technique for obtaining spectral and spatial information simultaneously is point projection spectroscopy. ${ }^{4}$ In this technique, one or two of the backlighting beams are focused onto one or two 15- to $20-\mu \mathrm{m}$ coated fibers, to produce a flash of quasi-continuous $x$-rays from a point source. This "point" projects a shadow of the object after the $x$-rays have been reflected off a Bragg crystal, forming an image. Because reflection only occurs when Bragg's law is satisfied, the shadow has spectral resolution as well as spatial resolution.

The $x$-ray laser researchers are characterizing the coherence, both spatial and temporal, of $x$-ray lasers, and beginning to demonstrate biological applications. The spatial coherence of laboratory $x$-ray lasers has been measured using a diagnostic based on partially coherent diffraction from a uniformly redundant transmission slit array. 5

\section{$\mathrm{X}$-ray Spectrometers}

In the crystal regime, there are several static survey instruments, called Henways, that use convex crystals at a distance of about 1 meter from the plasma for survey work from $1.5 \mathrm{keV}$ to about $10 \mathrm{keV}$.

There are also streaked crystal spectrometers. ${ }^{6}$ On the ten-beam target chamber, these use flat crystals on the front of a close-in $x$-ray streak camera at anywhere from 3 to 6 degrees Bragg angle, or at a much larger Bragg angle for high resolution. For implosion work there has been an emphasis on high-energy, highresolution spectroscopy because of the need for the line emission to penetrate the compressed pushers. Argon dopants have been successfully used to measure density from line broadening, but for future implosions, higher energy $x$-ray emission with higher spectral resolution will be required. With a large Bragg angle and a PET crystal, resolving powers of about 2000 have been achieved in streak mode at photon energies of about $6 \mathrm{keV}$.

For accurate absolute measurements of broad-band spectra, an array of fifteen calibrated $x$-ray diodes, called Dante, is used with filters and grazing incidence mirrors to isolate channels between $100 \mathrm{eV}$ and $>1.5 \mathrm{keV} .^{7}$ This was one of 
the core diagnostic systems of Nova, and although originally designed for 1-ns recording, the photocurrent is now commonly recorded with fast oscilloscopes (such as the Tektronix SCD 5000), giving a time resolution FWHM of 135 ps with some small ringing at $300-400$ ps. This allows time-resolved measurements of the $x$-ray conversion efficiency to be routinely made.

There are several soft $x$-ray instruments enumerated in Table III-1. A particularly highresolution grazing-incidence spectrometer, HIRES, can achieve a spectral resolution of 8,000 26,000 in the 100 - to $200-\AA$ range when used with an $x$-ray streak camera, and up to 35,000 when used with soft $x$-ray film. This instrument was recently used to measure the line width of the dominant laser transition in neon-like selenium $x$-ray laser plasmas, and to observe the scaling of the line width with amplifier gain-length product. 8

The Streaked Flat Field Spectrometer (SFFS) is a time-resolved XUV spectrometer that utilizes a Harada varied line space grating. ${ }^{9}$ The grating has a flat focal plane that lies almost perpendicular to the $\mathrm{x}$-ray propagation direction, allowing the use of a transmission photocathode $x$-ray streak camera. The instrument is sensitive from about $400 \AA(30 \mathrm{eV})$ to $\sim 10 \AA(1000 \mathrm{eV})$. With a 1200 -line-per-mm grating with high efficiency in higher orders, up to 6th order has been seen at $44.83 \AA$. In addition, the instrument is used to view short-wavelength $x$-rays, such as $44.83-\AA x$-ray laser emissions, in multiple orders, extending the dynamic range of the spectrometer and allowing a resolution of $\lambda \Delta \lambda \sim 500$ to be achieved in some measurements (e.g., $44.83 \AA$ in 5 th order).

For shorter-wavelength spectra, the SFFS can be used with a 2400 -line-per-mm grating. Both gratings are installed in the spectrometer and either can be selected by translating them into position. The wavelength range is determined by an iridium-coated mirror, midway between the detector and the grating and almost parallel to the grating surface. The mirror relays the spectrum from the grating to the photocathode. Unlike similar instruments that rely on moving the detector to vary the wavelength coverage, the SFFS keeps the detector fixed and moves the relay mirror. This feature allows the spectrometer to be re-entrant, increasing sensitivity as the spectrometer moves closer to the targets.

Like all the streaked diagnostics on the twobeam target chamber (Keanetech, XCSS, XRL power meter, XRL coherence measurement, SOS-3, SOS-5, PCDs, Dante, HSI, High Flux, UVImager, High Res, 2-Beam Incident Streak, Laser Diagnostic Station), the SFFS has a fiberoptic timing fiducial that allows the recorded spectra to be referenced to the incoming irradiation and other diagnostics to a precision of $\sim 30 \mathrm{ps}$. The timing fiducial also allows for the testing and timing of streak cameras without firing the entire laser chain.

The Microchannel Plate Intensified Grazing Incidence Spectrograph (MCPIGS) is a grazingincidence XUV spectrometer with a gated microchannel plate detector. The instrument is a Rowland circle geometry spectrometer with a $2^{\circ}$ angle of incidence on the $998.8-\mathrm{mm}$ radius grating. The detector is a $150-\mathrm{mm}$-long curved microchannel plate that lies along the Rowland circle. It has several configurations of strips that are gated off at intervals using a silicon Auston switch triggered by the fiducial laser beam. With seven gratings available, a broad range of $\mathrm{XUV}$ spectra can be surveyed with this instrument. There are two MCPIGS spectrometers on the twobeam chamber. One lies in the equatorial plane to view $\mathrm{X}$-ray laser output emitted in a narrow beam perpendicular to the plane of the Nova foci. The other MCPIGS spectrometer views the plasma from an off-axis direction.

\section{Optical Diagnostics}

The optical and UV diagnostics are also listed in Table III-1. One of the most ubiquitous diagnostics is the streaked optical imager, called the SOP (for streaked optical pyrometer). This instrument is an $f / 10$ Cassegrain telescope with a magnification of 8.47 , and a resolution that is close to diffraction-limited. The instrument is most commonly used to record shock breakout from witness plates, for which purpose the image is recorded onto a UV-sensitive streak camera. Alternatively, the image can now be recorded onto a gated optical imager.

There are several streaked optical spectrometers at fixed locations on the two target 
chambers (SOS1, SOS2, SOS3, SOS4 and SOS5). These are primarily used to measure the stimulated Brillouin and Raman spectra to derive density and laser-induced plasma turbulence levels. ${ }^{10}$ To make it possible to map out the angular dependence of the scattered light, a multichannel streaked spectrometer that allows the angular position of the detectors to be easily varied has been implemented on the Nova tenbeam target chamber.

For measurements of the absorption of laser light there is a set of energy balance modules for use on both the ten-beam and the two-beam target chambers.

\section{Hohlraum Radiation Sources}

As an overview of the capabilities of a laser system of this sort we provide the following. First, a unique feature of the system is the hohlraum. A hohlraum is, in the jargon of the Inertial Confinement Fusion (ICF) program, a high-Z-usually gold-cylinder with end caps. The laser enters the cylinder through the ends and is focused on the interior walls in an annular pattern. The laser light is converted to $x$-rays by laser-matter plasma creation. A typical hohlraum is $2400 \mu \mathrm{m}$ long and has an $800-\mu \mathrm{m}$ radius. The radiation temperature equivalent, found by measuring the emission from the hohlraum and equating it to $\sigma \mathrm{T}^{4}$, is $\sim 200 \mathrm{eV}$.

The temperature and the size and scale of the hohlraums can, of course, be changed to accommodate experimental needs. See Fig. III-1

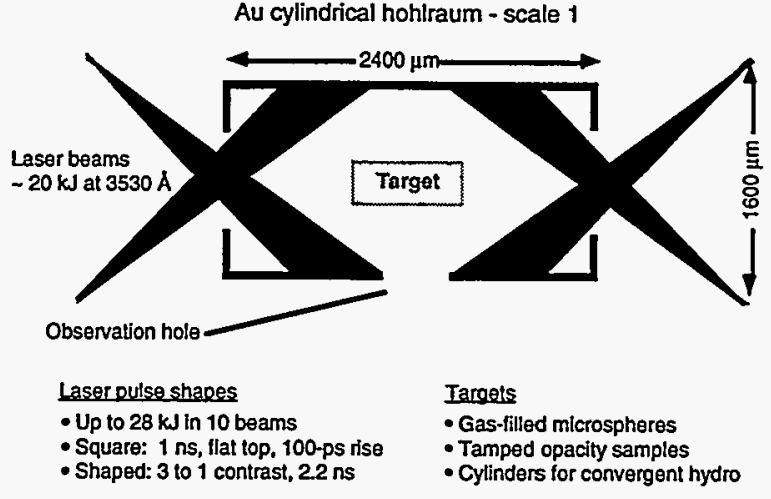

Figure III-1. Schematic view of a hohlraum. The one shown here is the nominal hohlraum. Called a scale- 1 , it generates a radiation temperature of $\sim 200 \mathrm{eV}$.

for an example of a hohlraum. The design of a hohlraum can affect the radiation temperature and conditions of the targets placed inside.

In Fig. III-2, three different types of hohlraums are shown, each of which has a different utility. Note that the radiation temperature is changed by the hohlraum scale. Figure III-3 shows the scaling of the measured radiation temperature vs incident laser energies. In the figure there is also a simple fit to the data using empirically determined parameters, the conversion efficiency of the laser energy to $x$-ray energy, $\eta$, and the amount of loss of laser energy due to heating of the walls, $\alpha$; these are determined to be 0.7 and 0.8 respectively for the

Scale 3
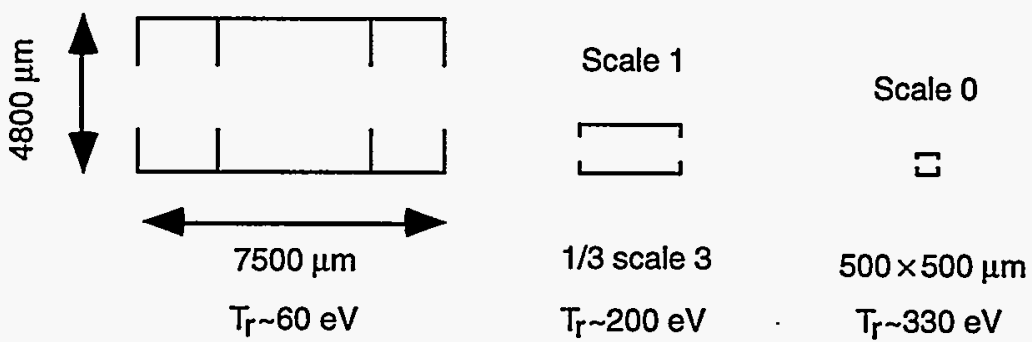

Figure III-2. Schematic view of several different hohlraums. The different types are used to create a radiation environment for various purposes. Numerous configurations exist for different tasks. 


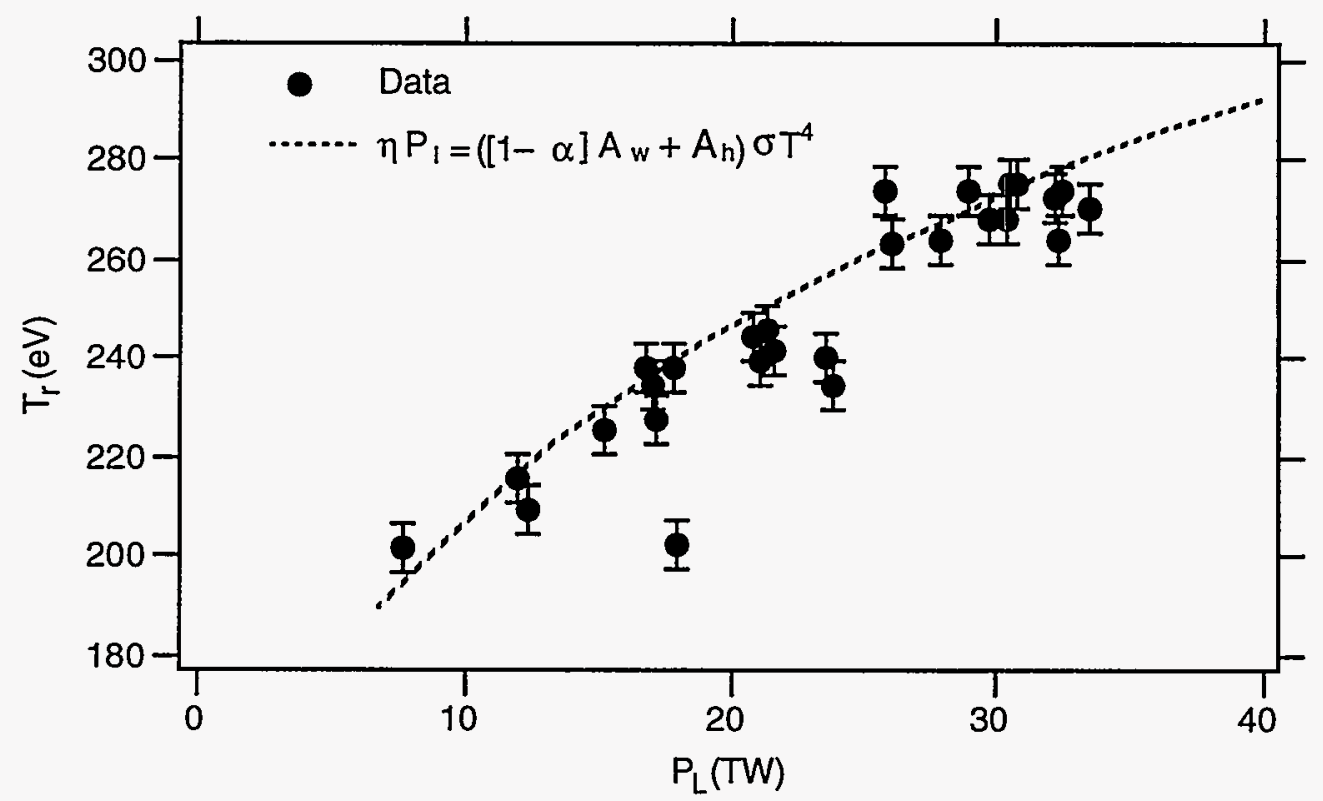

Figure III-3. Measured radiation temperature vs input laser power. Temperature is derived from equating the measured energy output to $\sigma \mathrm{T}^{4}$. The data points are indicated, along with a dashed line showing a simple fit to the data using empirically determined parameters.

scale-1 geometry. The two areas that play a role are the area of the walls, $A_{W}$, and the area of the holes in the wall, $A_{h}$. The formula is given as

$$
\eta P_{L}=\left([1-\alpha] A_{w}+A_{h}\right) \sigma T^{4} \text {. }
$$

The ten beams of the laser typically deliver $20-30 \mathrm{~kJ}$ of $0.35-\mu \mathrm{m}$ light into a cylindrical gold hohlraum that is $2.5 \mathrm{~mm}$ long and has a diameter of $1.6 \mathrm{~mm}$, producing a uniform quasi-thermal source of $\mathrm{X}$-rays. $\mathrm{X}$-ray drive produces high ablation pressures that are spatially very smooth. The radiation can be used to drive capsule implosions, drive planar shocks in material samples for hydrodynamics, provide a local thermodynamic equilibrium (LTE) environment for opacity studies, and provide drive for equation-of-state studies. The shock pressure created by radiation drive is a strong function of the temperature:

$$
\mathrm{P}(\mathrm{Mbar})=2.3 \times 10^{4} \mathrm{~T}^{3.5}(\mathrm{keV}) .
$$

For example, shocks of approximately $100 \mathrm{Mbar}$ can be driven in plastic with $200-\mathrm{eV}$ drive.

The laser is also used for direct illumination of targets. The direct illumination of low-density targets, for example foams or gases, can produce plasmas with an electron temperature of a few
$\mathrm{keV}$. Ablation pressures generated in directly driven targets are:

$$
\mathrm{P}(\mathrm{Mbar})=12\left[\mathrm{I}\left(10^{14} \mathrm{~W} \mathrm{~cm}^{-2}\right) / \lambda(\mu \mathrm{m})\right]^{2 / 3}
$$

Thus, for laser light with a wavelength of $\lambda=0.53 \mathrm{~mm}$ (frequency-doubled light) and an intensity of $I=2 \times 10^{14} \mathrm{~W} / \mathrm{cm}^{-2}$, the ablation pressure generated is about $10 \mathrm{Mbar}$. Directly driven shocks can be made reasonably planar over about $1 \mathrm{~mm}$ by smoothing the beam profiles with random phase plates and SSD.

To see the radiation environment of a hohlraum quantified, refer to Section VIII, Radiation Sources.

\section{B. NIF Facility Definitions}

The following definition of a proposed highenergy laser facility is based on ongoing work at LLNL. This facility will be capable of producing 1.8 MJ of temporally shaped laser energy on a variety of targets. This laser system is being developed by the LLNL ICF program, which provided much of this information. 


\section{Laser Beams}

The laser will have four separate beam-line bundles, each with a $4 \times 42$ array of beamlets, for a total of 192 beams. The laser is based on the multipass amplifier Nd:YLF configuration where the initial seed laser is generated in a desired temporally shaped pulse. Amplification occurs in a series of multipass and single-pass amplifiers separated by spatial filtering pinholes in increasingly larger diameter sections. The $1-\omega_{0}$ (i.e., $1.053-\mu \mathrm{m}$ ) wavelength beam arrives at the target chamber with approximately $10 \mathrm{~kJ}$ of energy in a beam $40 \mathrm{~cm}$ square; this assumes a pulse that is temporally a 5 -ns square pulse. A variety of other pulse shapes will be available at other energies.

The beam is then frequency doubled using a KDP crystal to a 2- $\omega_{0}$ (green beam) and tripled, to a $3-\omega_{0}$ (blue beam), for final focusing onto the target. The crystal conversion efficiency will yield approximately $10 \mathrm{~kJ}$ of $3-\omega_{0}$ energy in the beam to be focused into the target chamber. The 192 beams will be balanced to produce no more than $8 \% \mathrm{rms}$ deviation in power.

The 1- $\omega_{0}$ beam will be of high spatial uniformity; however, the nonlinearity of the up conversion to $2 \omega_{0}$ or $3 \omega_{0}$ amplifies any amplitude modulation in the beam. Several techniques can be used to smooth the beam, a major consideration for directly irradiating a surface where uniformity is desired. First, a random phase plate can be used to introduce a randomness in the phases of the beam. This creates an effectively smoother intensity distribution by overlapping many small beamlets of random phase created from the large aperture beam. Second, the beam can be split up into several pieces using wedges which allow an effectively flat intensity distribution over the focal spot. Third, a bandwidth can be introduced on the normally monochromatic beam; when combined with a diffraction grating, this will be used to move the focal spot, resulting in a temporally smoother intensity.

On the NIF, temporal smoothing will also be achieved by overlapping four separate wavelengths, separated by $5-10 \AA$, and smoothing each one by spectral dispersion (adding bandwidth).
The limiting factor in the firing of the beam is the cooling-down time for the large disk amplifiers. This limits a particular laser beamline from firing more than once every eight hours. Diagnostic setup and target alignment will be performed within this time.

\section{Beam Pointing, Aiming, and Synchronicity}

The beam as it enters the chamber is focused into the target chamber center using an $f / 8$ lens that will produce a minimum spot size of $500 \mu \mathrm{m}$ in diameter.

The accuracy of the focusing, or pointing, will be $\pm 50 \mu \mathrm{m}$ rms, with a maximum displacement of the beam centerline from the target chamber center of $7 \mathrm{~cm}$. The beams come into the chamber in 48 clusters of four beams, with half entering on each of opposite sides of the midplane. Multiple laser beams will enter on each side of the hohlraum along two concentric cones, with cone half-angles of approximately $27^{\circ}$ and $53^{\circ}$, and with two-thirds of the beams on the outer cone and the remaining one-third on the inner cone. The pulse shape can be different on the two cones.

\section{Laser Pulse Shapes}

There will be a flexible pulse-shaping capability, so that the energy can be delivered in up to a 220-ns shaped pulse with $>50: 1$ intensity contrast between peak and foot of the pulse. Approximately $20 \%$ of the beams can be delayed, independently of each other, by up to $30 \mathrm{~ns}$ after the main beam. This last capability will be useful for providing a source of backlighter $\mathrm{x}$-rays.

\section{Laser Shot Rate}

The NIF will have a capability of 3 shots $/ 24$ hours for 100 shots/year with energy of $100 \mathrm{~kJ}$, $35 /$ year for energy of $\mathrm{MJ}$, and 10 /year for energy of $20 \mathrm{MJ}$.

\section{Beam Diagnostics}

There will be several diagnostics of beam performance available. First, the pulse shape will be monitored in the master oscillator for temporal signature and energy. Next, the 
propagated beam can be monitored for the total $1-\omega_{0}$ energy and the temporal variation of the 1- $\omega_{0}$ energy. Further, images can be made of the near and far field of the $1-\omega_{0}$ beam.

The up-converted beam, in either $2 \omega_{0}$ or $3 \omega_{0}$, can be monitored in the chamber for absolute as well as temporal intensity, and images of the beam front can be produced in the far or near field.

\section{NIF Facility Modifications}

A central issue that was addressed by the scientific community was the desired modifications to the original NIF design. To a large degree the requests of the scientific community were very similar to those of other communities. In particular, the group desired smaller spot sizes and pulse variation from 100 ps or less to $20 \mathrm{~ns}$. In addition, they identified as important the need to establish line foci to perform $x$-ray laser experiments and the need to have an expanded subpicosecond capability. However, the most important request by far was the many demands for improved turnaround times. This took many forms, but the message was the same-a science-based universityintensive effort that seeks to train students requires more than one to three experiments a day. The suggestions to improve the turnaround included:

- Additional target areas.

- More rapid cooling of the amplifiers.

- More independence of the beams, so that a portion of the beams could be used flexibly.

- The introduction of a smaller (e.g., Novasized) beam for independent backlighting.

Each of the special requirements that the scientific community asked for is presented below with a brief discussion of the requirement.

\section{Higher Beam Irradiance Requirements}

Some physics experiments will require higher beam irradiances than those outlined in the NIF design documents. The present laser spot diameter requirement for ignition targets, utilizing kinoform phase plates for beam conditioning, is $500 \mu \mathrm{m}$. This will be achieved with adaptive optics with a number of actuators consistent with this requirement. The NIF Functional Requirements and Primary Criteria specify the maximum spot size to be $500 \mu \mathrm{m}$. For higher irradiances that will lead to increased hohlraum temperatures and the subsequent pressures desirable for a subset of the science experiments, smaller spot sizes are needed -50 to $150 \mu \mathrm{m}$, or as small as possible and practical (see the discussion in Section VII, Plasma Physics). Higher irradiances than for the present design may be obtained by increasing the number of actuators in the adaptive optic. To facilitate pointprojection $x$-ray backlighting, beams focused to the minimum spot size should be able to effectively irradiate a $25-\mu \mathrm{m}$-diameter fiber or equivalent.

\section{Variations in Lens-Focusing Requirements}

The NIF should have the capability of putting cylindrical lenses on at least 20 beamlets for experiments utilizing $x$-ray lasers. These lenses should nominally produce a focus of $\sim 150 \mu \mathrm{m} \times 5 \mathrm{~cm}$. These lenses should be capable of being easily inserted and removed. The beamlets should also be able to operate at $2 \omega$ at power levels consistent with those required for $\mathrm{x}$-ray lasers.

\section{Target Chamber Insertion Mechanisms}

The target chamber should have two target insertion mechanisms. The second target inserter does not have to have cryogenic capability.

\section{Added Target Alignment Capabilities}

The scientific community envisions the use of targets that may be, in general, more complicated than ICF targets, so additional target alignment capabilities may be needed. This is most simply specified by requiring the capability to view all sides of the targets(s) during target alignment.

\section{Reduced Time Between Experiments}

To accommodate the expected large number of users, the time between shots should be 
reduced below the maximum specified eight hours. The facility should incorporate the improvements that are developed from these programs to ensure greater access to the user communities.

\section{Flexible Time Intervals Between Experiments}

Finally, it is important to note that the largest possible timing and pulse duration flexibility needs to be incorporated for the NIF to reach full utilization. Individual beamlet pulse-length flexibility, as presently designed, is highly desirable, with range-of-pulse widths from $<0.1 \mathrm{~ns}$ to $>20 \mathrm{~ns}$. NIF design should permit each cluster of four beams to be independently timed, with arbitrary time delays among the clusters. The time interval between the first and last beams should be $>200 \mathrm{~ns}$. If possible, delays of up to 4000 ns would be useful to some users.

\section{Short-Pulse Beams}

There was a decided interest in having the NIF contain as many short-pulse beams as possible. Because of its size, the projected single short-pulse beam was considered interesting enough to warrant involvement by the community. However, scientists interested in the short-pulse experiments felt that a single beam was far too limiting. As can be witnessed from the discussions of short-pulse experiments in both Section VII, Plasma Physics, and Section VIII, Radiation Sources, there are a large number of topics in this category, and the possibility that one or more may become of primary interest in the future is thought by the participants to be rather large. Hence, additional short-pulse beamlines would be strongly supported.

\section{References}

1. J. D. Kilkenny, Laser and Particle Beams 9, 49 (1991).

2. J. D. Kilkenny, Phys. Fluids B 2, 1400 (1990);

B. A. Remington, et al., Phys. Rev Lett. 67, 3529 (1991).

3. R. J. Ellis et al., Rev. Sci. Instr. 61, 2759 (1990).

4. J. Balmer et al., Phys Rev. A 40, 330 (1989); T. S. Perry et al., Phys. Rev. Lett. 67, 3784 (1991).

5. J. E. Trebes et al., Phys. Rev. Lett. 68, 588 (1992).

6. B. A. Hammel et al., Rev. Sci. Instrum. 61, 2774 (1990).

7. H. N. Kornblum et al., Rev. Sci. Instrum. 57, 2179 (1986).

8. A. Koch et al., Phys. Rev. Lett. (1992).

9. T. Kita et al., Appl. Opt. 22, 512 (1983).

10. R. E. Turner et al., Phys. Rev. Lett. 57, 1725 (1986). 
This page intentionally left blank 


\section{Section IV}

\section{Astrophysics and Space Physics}

The level of feasibility of possible highenergy laser-based experiments discussed here ranges from a straightforward extrapolation of well-established Nova techniques, to rather speculative possibilities that will probably require technology beyond the presently proposed NIF capabilities before useful results can be obtained. Nevertheless, we discuss in this section the important scientific issues in approximately descending order of feasibility of application on the NIF facility.

\section{A. Opacity}

The radiative opacity of the material in stellar interiors plays a key role in determining how stars evolve-what the maximum mass of a stable star is, how hot and how luminous the star is while it burns its hydrogen fuel, what pulsational instabilities it may fall prey to, whether it loses much of its outer envelope in outbursts of one kind or another, and during which stage of its evolution it might explode as a supernova. The star's energy budget dictates these other consequences, and because the radiative flux is inversely proportional to it, opacity sets the flow of heat through the star. In particular, the instability of the star is especially sensitive to nuances in opacity, and is most sensitive in the temperature range of a few hundred thousand degrees Kelvin. Opacity is very complex in this region, and has stood as a formidable challenge to theoretical atomic physics. A brief discussion of recent advances is presented in Section VI, Material Properties, Part B.

Some of the regimes of temperature and density for which a star's structure and evolution are very sensitive to opacity are reachable in a laboratory high-energy-density facility such as the NIF, as illustrated in Fig. IV-1-temperatures from tens of electron volts to several hundred electron volts, and densities from normal solid density to a few hundred times less.

The figure shows two regimes possible on the NIF, one with the laser only (shown in light gray) and the other using ignition within a deuteriumtritium capsule (shown in black). These are compared with conditions for four phases within stars (shown in dark gray): hydrogen burning; helium burning; carbon burning; and neon, oxygen, and silicon burning. The phases shown progress toward increasing temperature because the Coulomb barrier that keeps the nuclei apart gets higher as the nuclear charges increase.

This progression toward increasing temperature is also an evolutionary sequence for stars. A star first gets hot enough in its interior to burn hydrogen (the main sequence starts), and only when the hydrogen is used up does the interior get heated and compressed sufficiently to ignite helium burning. At this point the star may be a red giant.

When the helium is exhausted, the interior again is heated and compressed until carbon burning begins via a suite of reactions beginning with ${ }^{12} \mathrm{C}+{ }^{12} \mathrm{C} \rightarrow{ }^{24} \mathrm{Mg}$ as well as other possible products. Among these products are $\alpha$ particles, which can be captured by the ${ }^{12} \mathrm{C}$ to build up a number of other nuclei, including oxygen, neon, and silicon.

When the carbon is exhausted and the star is a red or blue supergiant, it may undergo a phase of burning in which the ${ }^{20} \mathrm{Ne}$ is photodissociated to produce oxygen and $\alpha$ particles; the $\alpha$ particles are quickly captured again by the nuclei, and the 


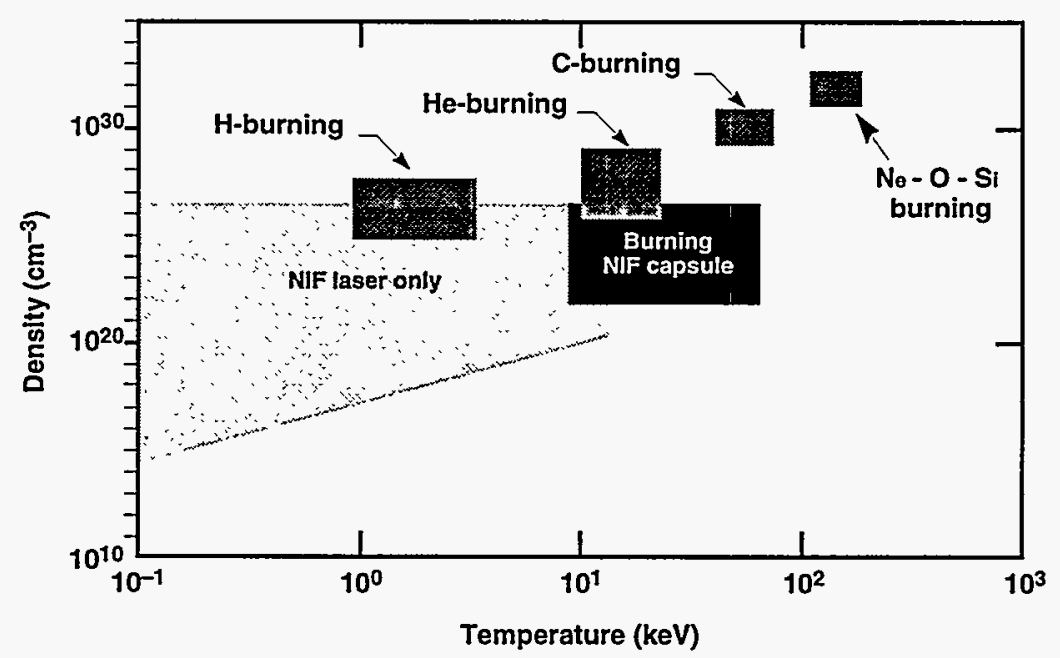

Figure IV-1. Comparison of the regimes of temperature and density that will be attained on the NIF with typical conditions found in stars as they progress through their successive nuclear burning stages. Two NIF regimes are shown. The broader regime (light gray) is for the NIF laser only, and extends up to about $14 \mathrm{keV}$. It shows the temperatures that may be reached in a laser-heated hohlraum on the NIF, or within an imploding capsule, even if there is no nuclear ignition. The second NIF regime (in black) shows the conditions that might exist in a deuterium-tritium capsule after ignition, with temperatures between between 9 and $60 \mathrm{keV}$. The dark gray areas show conditions within stars for four phases: hydrogen burning; helium burning; carbon burning; and neon, oxygen, and silicon burning. These phases progress toward increasing temperature. Although it doesn't always happen, this sequence of phases is also an evolutionary sequence for stars, from an initial hydrogen-burning phase, through the helium-burning and carbon-burning phases, to a phase where neon, oxygen, and silicon are burned.

oxygen reacts with itself to make a number of elements between magnesium and sulfur. This is indicated by the neon-oxygen-silicon box.

Whether this last phase occurs in a given star, or even whether carbon burning occurs nonexplosively at all, differs from star to star. In lower mass stars the nuclear evolution is cut off by a hydrodynamic instability that causes all the star's outer layers to be expelled, after which the star lives out its life as a white dwarf. In massive stars a thermonuclear instability turns the star into a supernova after the carbon-burning phase, or perhaps after the oxygen-burning phase.

The radiative opacity of interest in stars is a harmonic mean of the spectral-dependent opacity, which means that the opacity of interest is dominated by those windows in the frequency spectrum that lie between the strong absorption features. The kind of experiment that addresses the opacity is a spectral absorption measurement of a thick sample, centered on these frequency windows. The ability of the NIF to provide a stable temperature environment for a long enough period, combined with high-quality timeresolved $x$-ray spectrometers, makes it perfect for this kind of experiment.

The variation of temperature and density with depth for some detailed stellar models is shown in Fig. IV-2, which illustrates the structures for stars in late stages of evolution. Four late stages are shown: a possible MACHO (massive compact halo object), a 1-solar-mass white dwarf, a 25-solar-mass pre-supernova, and a star undergoing core carbon burning as well as helium and hydrogen burning. 


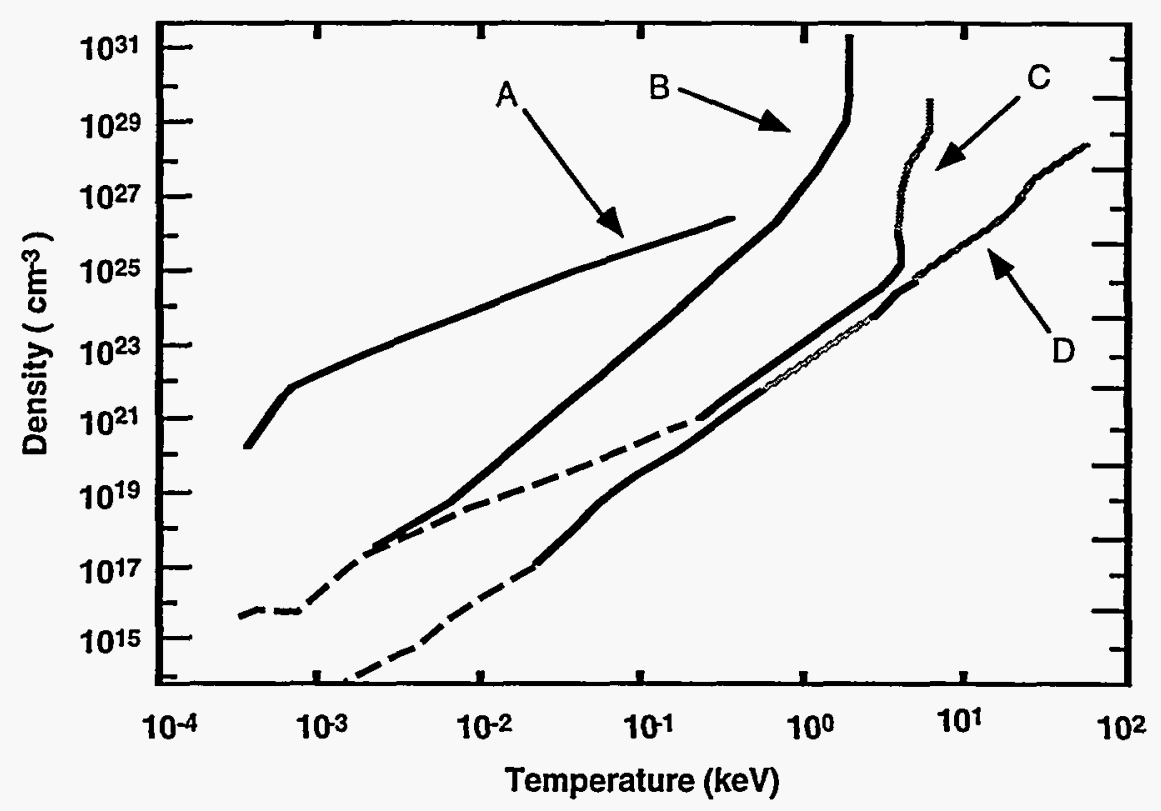

Figure IV-2. Variation of temperature and density with depth for models of stellar structure for four late phases. A) A possible MACHO-massive compact halo object. B) A 1-solar-mass white dwarf. C) A 1-solar-mass star at the tip of the red giant branch. D) A 25-solar-mass pre-supernova, a star undergoing core carbon burning as well as helium and hydrogen burning. Each curve shows several different dominant energy mechanisms-the dashed line for convective transfer, the solid line for radiative regions, and the gray line for regions in which the hydrogen has been completely depleted by nuclear burning.

Opacity has the most effect on stellar structure in the intermediate zone between the convective outer envelope and the hydrogendepleted core. The temperature here ranges from $10^{6}$ to $10^{8}$ degrees $\mathrm{K}(100 \mathrm{eV}$ to $10 \mathrm{keV}$ ) depending on the position in the star and the stellar mass. The corresponding range of density is from $10^{-4}$ to $10^{2} \mathrm{~g} / \mathrm{cm}^{3}$.

The stellar material is rich in hydrogen and helium, which do not dominate opacity in these ranges, but there are also significant amounts of carbon, nitrogen, oxygen, and iron. The carbon, nitrogen, and oxygen ions are important contributors at $100 \mathrm{eV}$, but iron is very important over the whole temperature range. A first measurement of the opacity of iron has already been made with Nova, as described in Section VI, Material Properties, Subsection B. With the NIF, the temperature range up to a few $\mathrm{keV}$ without ignition, or to more than $10 \mathrm{keV}$ with ignition, will be available, which means that conditions at the centers of the stars will be reachable.
Another area in which NIF-based experiments could be useful is with regard to opacities and $x$-ray cross-sections relevant to compact $x$-ray sources such as cataclysmic variables, $x$-ray binaries, and quasars. Direct measurements of inner- and outer-shell photoabsorption cross-sections of carbon, nitrogen, and oxygen could be made in well-characterized laser-produced plasmas via a backlighter method. These cross-sections have not been measured in other ways because of the difficulty of producing a sufficiently large column density of the desired ions. Such measurements would complement theoretically calculated crosssections and aid in the modeling of $x$-ray ionized material in and around compact sources.

In the last few years a group at LLNL has made new calculations of the radiative opacity for stellar interiors, and this work has been incorporated into theoretical models of stellar evolution and pulsation by a number of astrophysicists around the world. As a result, 
some outstanding astrophysical problems have been solved.

The problems solved are ones that appeared when stellar structure and evolution calculations made with the tables of opacity generated using the then state-of-the-art opacity codes at LANL were compared with observations. Previous stellar evolution calculations for the most massive stars had predicted that the range of surface temperature for the hydrogen-burning phase of evolution was narrow-that the stars were all quite hot at this time, but lower temperatures were observed. With the new opacity calculations, the range of temperatures predicted is extended to lower temperatures as observed.

When the massive stars evolve from blue supergiants to yellow supergiants they pulsate for a while as Cepheid variables. The former evolution calculations led to a relation between stellar luminosity and mass that gave the wrong pulsation periods; this problem is solved with the new opacities. The evolution calculations of the low-mass stars in globular clusters formerly led to inferred ages for the clusters that were greater than the age of the universe; with the new opacities, this problem has now disappeared. Even though these and other pieces of astrophysical evidence support the new LLNL opacities, the opacities embody many approximations, like the LANL opacities from the 1960 s and 1970s that preceded them. They should be confirmed in as many ways as possible, including by direct experiment.

\section{B. Equation of State}

Stellar structure and evolution are governed by three physical properties of the star's material-the opacity just discussed, the nuclear reaction rates, and the equation of state. The last of these means the relations between the density and temperature of the material on the one hand and its pressure and internal energy, specific heats, etc., on the other hand. Under many circumstances, the equation of state in stellar interiors is quite simple-most of the gas consists of hydrogen and other light elements, and these have lost most of their electrons. Furthermore, the gas density is low enough that the atomic ions seldom interact, so ideal gas physics is a good approximation.

This situation is a happy one for the astrophysicist, but unfortunately it is not always the case. In the very centers of stars, particularly those in the later stages of their evolution, density is quite high and the plasma becomes strongly coupled. That is, the various ions interact strongly and no longer behave as free particles. This is often accompanied by electron degeneracy - the plasma electrons tend to fill up the states allowed by Pauli's exclusion principle, which forces some electrons to become very energetic. These energetic electrons dominate the pressure and internal energy; however, the ions still play a major role in the specific heats.

The theory of stellar evolution is affected by uncertainties in the equation of state in a few areas. The white dwarf stars are the remnantsthe nuclear ashes-of stars that have run through most of their evolution and have at some point ejected their outer layers containing all the unburned nuclear fuel. They are the compressed former cores of their parent stars. A white dwarf of the sun's mass is the size of the earth. With no more nuclear heat source, the white dwarf simply cools off, a process that takes many millions of years.

Through most of the white dwarf, the pressure of degenerate electrons supports the material against gravity, but near the surface the electrons become less degenerate and the ions become important. The ions are also important in setting the specific heat and thus the rate at which the white dwarf can cool.

White dwarfs are also sometimes pulsationally unstable. Pulsation is a process that is sensitive to specific heats, and indeed white dwarfs are often in several pulsation modes simultaneously. The precise measurement of the pulsation frequencies gives a sensitive probe (asteroseismology) of the interior structure of the star. 
See Fig. IV-3 for an example of the importance of improved opacity and equations of state on the understanding of stellar evolution. The figure shows the effects of opacity on Cepheid pulsations. Until the early 1990s, there remained a discrepancy between the predicted mass of pulsating Cepheid stars and observation. The mass should be predicted from the ratio of the first harmonic, $\mathrm{P}_{1}$, to the fundamental, $\mathrm{P}_{0}$; however, until the opacity of the stars was corrected the discrepancy remained. The figure shows calculated results both with older opacities (upper, lighter lines) and with opacities corrected (solid lines), and the observed ratios (dots).

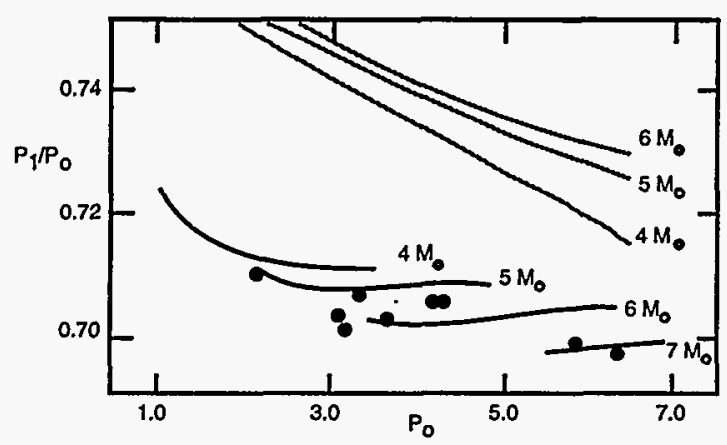

Figure IV-3. Effects of improved opacity and equations of state on understanding Cepheid pulsations. Until the early 1990s, there remained a discrepancy between the predicted mass of pulsating Cepheid stars and observation. The mass should be predicted from the ratio of the first harmonic, $\mathbf{P}_{1}$, to the fundamental, $\mathbf{P}_{\mathbf{0}}$; however, until the opacity of the stars was corrected the discrepancy remained. The ratio of $P_{1} / P_{0}$ is presented as a function of the fundamental $\mathbf{P}_{\mathbf{0}}$. The upper (lighter) lines represent the results with the older opacities, the solid lines are the older results with the opacity corrected, and finally, the dots are the observed ratios. The results of the comparison indicate that the new opacities resolve the problem, putting the observations and the theory in agreement. The ability to verify opacity at astrophysically relevant conditions will be greatly enhanced with the NIF.
The results of the comparison indicate that the new opacities resolve the discrepancy, putting the observations and the theory in agreement. The ability to verify opacity at astrophysically relevant conditions will be greatly enhanced with the NIF.

Another kind of dwarf star-the brown dwarf-is poorly understood, owing in part to our imperfect understanding of the equation of state. Brown dwarfs are stars with masses so low that they never get hot enough inside to burn hydrogen, but simply condense from the interstellar medium and shine dimly for awhile as they get rid of the heat formed in condensing. Brown dwarfs are very difficult to detect, but it is possible that they are so numerous that they comprise as much as half of all the mass in our galaxy. This is mass that is inferred to exist from the dynamics of the galaxy, but that has not been discovered in luminous form. Their internal structure and their cooling time depend on the details of the equation of state at densities approaching solid density and temperatures of a few $\mathrm{eV}$; these conditions are easily reached in a laser experiment.

Equation-of-state (EOS) experiments fall into two classes. One class might be thought of as "direct" EOS experiments, in which for a certain experimental setup the experimental data yield a quantity that can be compared with the tabulated functions of pressure, energy, etc. The second class is of "indirect" EOS experiments, which measure some of the detailed intermediate data that enter into theoretical expressions for the thermodynamic functions. Experiments of the second kind serve as benchmark tests of the EOS theory. Experiments of the first kind are described in detail in Section VI, Material Properties.

The indirect kind of experiment could, for example, seek to validate the model of plasma screening-very important in dense plasmas-by diagnosing the occupation probabilities of atomic states close to the ionization limit. This is discussed at greater length in Section IX, Radiative Properties. 


\section{Plasma Spectroscopy in High-Energy Astrophysics}

High-energy astrophysics refers to $x$-ray and gamma-ray, and to some extent optical and UV, observations of stars and galaxies for which the source of the radiation is a violent event, or a plasma very far from thermodynamic equilibrium. Among these are quasars and other exploding galaxies, and neutron stars that are ingesting material from a companion star. The technique used to study them is to investigate their spectra-in the $x$-ray or gamma-ray range, or whatever is available- to find the signatures that reveal the nature of the emitting object. The astronomer's problem is to obtain a spectrum showing as much detail as possible. However, a second problem is to have the knowledge base on non-equilibrium spectra that allows inferences to be drawn from the data. Here is where the NIF has a role, working together with first-principles plasma-modeling tools.

An area where our modeling of plasma is relatively weak concerns low-temperature radiation-dominated plasmas. These are plasmas irradiated by dilute radiation sources-that is, the illuminating radiation field is much weaker in absolute intensity than the blackbody that matches the spectral shape. The radiation, while strong in absolute terms, is still weak in comparison with the very high implied temperature. Such plasmas are very far from local thermodynamic equilibrium (LTE). One uncertainty is of the plasma energetics. Perhaps a lot of energy is tied up in states that are not evident in the spectrum.

\section{The Bowen Mechanism}

Because the plasmas are radiationdominated, the thermodynamic state of the matter and the appearance of the spectrum itself depend strongly on the flow of radiation through the plasma. The re-absorption of radiation emitted elsewhere plays a major role. A particularly notable example of this is the Bowen fluorescence mechanism, which is discussed in some detail below and shown schematically in Fig. IV-4.

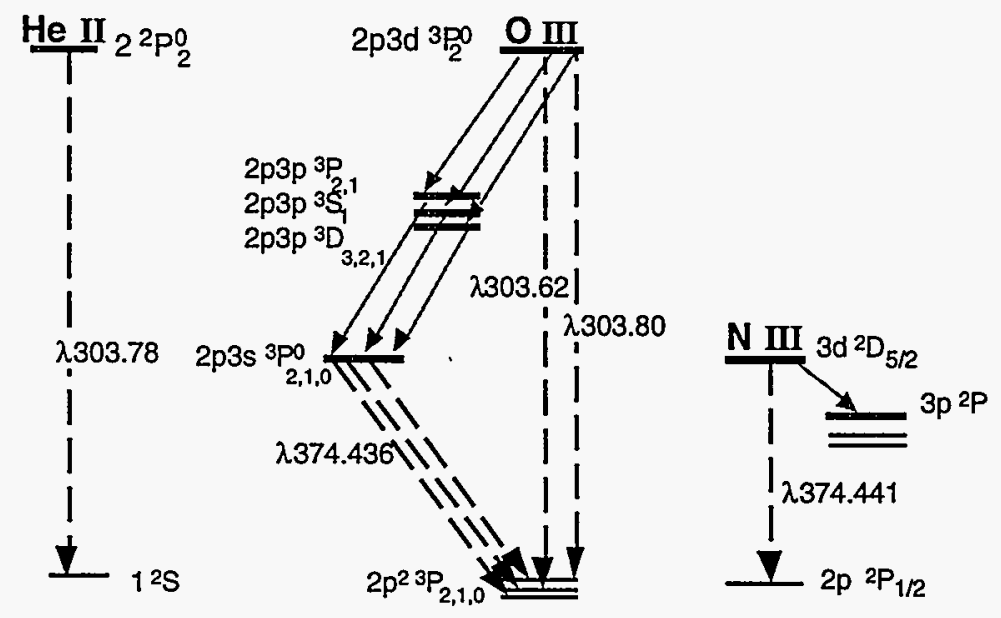

Figure IV-4. Grotrian diagram for the Bowen mechanism. The observed fluorescence occurs when the emission from the HeII Lyman $\alpha$ transition pumps the $2 \mathrm{p}^{2}{ }^{3} \mathrm{P}$ state of OIII to the state $2 \mathrm{p} 3 \mathrm{~d}{ }^{3} \mathrm{P}$. This leads to the enhanced emission from the transition down to the states $2 p 3 p{ }^{3} P,{ }^{3}$, and ${ }^{3} D$. There is also enhanced emission from these states to the $2 \mathrm{p} 3 \mathrm{~s}^{3} \mathrm{P}$ level. In addition, it has been pointed out that the emission from the OIII $2 \mathrm{p}^{3} \mathrm{~s}^{3} \mathrm{P}_{2}$ to the $2 \mathrm{p}^{2}{ }^{3} \mathrm{P}$ can pump the ground state of NIII $2 \mathrm{p}{ }^{2} \mathrm{P}$ to the $2 d^{2} D_{5 / 2}$ level, which then can decay via the $3 p^{2} P$ level with enhanced emission in the visible. This latter part of the mechanism is not yet confirmed. The NIF would be required in order to generate both the large volumes of plasma necessary and a radiation source sufficient to create the plasma in an appropriate state to provide experimental verification of the Bowen mechanism. 
Ira Bowen found, in 1924, that in some nebulae (the planetary nebulae that are shells ejected by red giant stars) the emission lines of OIII arising from the upper states $2 \mathrm{p} 3 \mathrm{~d}^{3} \mathrm{P}_{2}$ were greatly enhanced relative to other members of the same multiplet. The lower levels of these transitions, namely $2 \mathrm{p} 3 \mathrm{p}{ }^{3} \mathrm{P}_{2,1}, 2 \mathrm{p} 3 \mathrm{p}{ }^{3} \mathrm{~S}_{1}$, and $2 \mathrm{p} 3 \mathrm{p}{ }^{3} \mathrm{D}_{3,2,1}$ fed transitions to $2 \mathrm{p} 3 \mathrm{~s}^{3} \mathrm{P}_{3,2,1}$ that were also enhanced. Bowen found that there was a coincidence between the HeII Lyman $\alpha$ line at $\lambda 303.78$ and the OIII line $\lambda 303.80$ that excites $2 \mathrm{p} 3 \mathrm{~d}$ ${ }^{3} \mathrm{P}_{2}$. Furthermore, the decay of $2 \mathrm{p} 3 \mathrm{~s}^{3} \mathrm{P}_{1}{ }_{1}$ produces $\lambda 374.436$, which can pump the line $\lambda 374.441$ in NIII that excites $3 d^{2} D_{5 / 2}$, producing still more fluorescence.

The NIF would be required in order to generate both the large volumes of plasma necessary and a radiation source sufficient to create the plasma in an appropriate state to provide experimental verification of the Bowen mechanism. The uniform heating of material to extreme conditions, when the plasmas require $x$-ray heating, is energy-intensive, because large proportions of the flux are needed for volumetric heating.

The Bowen mechanism was analyzed in the 1960 s and 1970s, not with a full collisionalradiative model, but with some radiative transfer. The NIII branch is not considered wellestablished, but at least the first lines in the OIII branch are. The line coincidences have mismatches of $16 \mathrm{~km} / \mathrm{s}$ between HeII and OIII, and $4 \mathrm{~km} / \mathrm{s}$ between OIII and NIII. The HeII/OIII difference is larger than the Doppler width, which is about $9 \mathrm{~km} / \mathrm{s}$ for helium at $20,000^{\circ} \mathrm{K}$. Most nebulae also have an internal velocity dispersion of about $10 \mathrm{~km} / \mathrm{s}$ due to general expansion, or to turbulence, and there is opacity broadening, especially in the HeII Lyman $\alpha$ line. These two factors combine to ensure coincidence.

The OIII lines, at $\lambda \lambda 3100-3400$, are seen in $\mathrm{x}$-ray binary stars like Sco X-1 (a low-mass $\mathrm{x}$-ray binary) and AM Her (a cataclysmic-variable-like white dwarf binary), and also in some Seyfert galaxies (spiral galaxies that are relatively ordinary but with a black-hole-like object in the center that makes $x$-rays and excites a lot of line emission in the nearby interstellar gasmini quasars).
The Bowen mechanism still does not have a secure theoretical footing, and needs some experimental verification. The full network of atomic kinetic rates connecting these levels of HeII, OII and NIII is complex enough to be hard to calculate. An experimental exploration of the Bowen mechanism will create a large volume of plasma with a temperature of $2-3 \mathrm{eV}$ and in which oxygen and nitrogen are mostly doubly ionized. This may be illuminated by a second region of hotter plasma that serves as a light source for the first. The column density of the large plasma, perhaps $10^{20} \mathrm{~cm}^{-2}$, would be ample to provide the necessary optical depth in Hell Lyman $\alpha$, and perhaps also in OII $\lambda 374.436$ as well.

\section{Low-Mass X-ray Binaries}

Low-mass $x$-ray binaries (LMXRB) consist of a neutron star in orbit with a 1- to 2-solar-mass star; the $x$-ray spectra are relatively hard-10 keV or so. Considerable modeling work has been done on these, with particular attention to the iron spectrum-first the K-shell lines, and more recently the L-shell emission. This is a formidable task, especially the dielectronic recombination, and experimental confirmation is very desirable. The iron $K$ and $L$ lines are seen in spectra that have been obtained of $\mathrm{X}$-ray binaries like Sco X-1 using BBXRT and two Japanese satellites. Similar lines are hinted at in the few decent $x$-ray spectra of Seyfert galaxies, which, if confirmed, would corroborate the theory of an accretion disk surrounding a black hole in the nucleus of the galaxy.

Figure IV-5 illustrates the relevant process of dielectronic recombination. The dielectronic recombination process is shown by the two arrows. A free electron in the 2 s continuum (on the left), whose energy is indicated by the blue line, is captured into one of the 2pnl states (on the right) at exactly the same energy, as shown by the first arrow. This state can decay by ejecting the free electron again, or, as shown by the second arrow, by radiating a photon and making the transition to a singly excited $2 \mathrm{snl}$ state, typically with the same $n$. This two-step process is dielectronic recombination. 


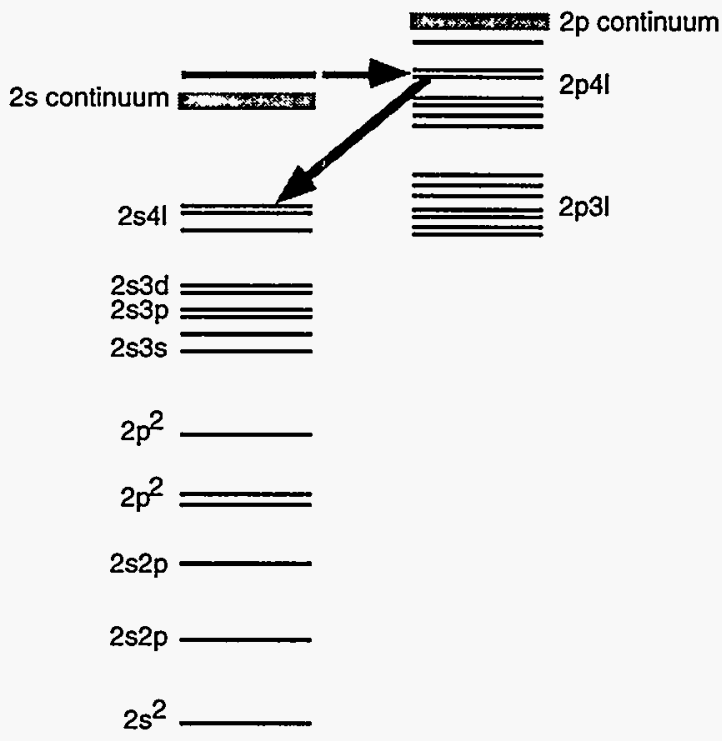

Figure IV-5. Illustration of the process of dielectronic recombination for the recombination of $\mathrm{C}^{+3}$ to $\mathrm{C}^{+2}$. On the left side are the levels of $\mathrm{C}^{+2}$ converging to the series limit, which is the $2 s$ state of $\mathrm{C}^{+3}$. Most of these levels belong to the configurations $2 \mathrm{snl}$, with $\mathrm{n}=3$, $4, \ldots$. On the right side are the levels of $\mathrm{C}^{+2}$ that converge to the $2 \mathrm{p}$ state of $\mathrm{C}^{+3}$. These belong to the configurations $2 \mathrm{pnl}$, again with $\mathrm{n}=3,4, \ldots$. Because the $2 s$ and $2 p$ states of $\mathrm{C}^{+3}$ have the same principal quantum number, their energy difference is slight, and therefore many of the 2 pnl states lie below the $2 \mathrm{~s}$ series limit and some of the 2pnl states lie just barely above the $2 s$ limit. The dielectronic recombination process is shown by the two arrows. A free electron in the $2 s$ continuum, whose energy is indicated by the blue line, is captured into one of the 2pnl states at exactly the same energy, as shown by the first arrow. This state can decay by ejecting the free electron again, or, as shown by the second arrow, by radiating a photon and making the transition to a singly excited $2 \mathrm{snl}$ state, typically with the same n. This two-step process is dielectronic recombination.

When the available doubly excited states lie high up in the ground-state continuum, the number of free electrons with the right energy is sharply limited by the Boltzmann distribution unless the electron temperature is high. In cases like the one illustrated, though, the $\Delta n=0$ transition of the upper ion allows doubly excited states very close to the ionization limit, which makes them accessible at even quite low electron temperatures. The total rate of dielectronic recombination for ions like this is very large.

In the next five years there will be two new high-resolution $x$-ray astronomical observatories: $X M M$ and AXAF. These will, for the first time, obtain spectral detail of the iron $K$ and $L$ features sufficient to distinguish different types of excitation. The line shapes in these objects, and, for the binaries, their time variations, can be analyzed to provide information about the radial and azimuthal structure of their accretion disks, provided the atomic model itself is sufficiently well understood.

An experimental approach to the iron $\mathrm{L}$ spectrum would be of great interest. The problem, in laboratory sources, is to avoid large optical depths in these lines, which is undesirable when basic line intensity data are being sought. The laser-produced plasma has the advantage over other sources, such as the Electron Beam Ion Trap (EBIT), in that the temperature can be kept as low as the astronomical situation indicates.

One of the key issues involving iron is the dielectronic recombination through the nearthreshold resonances that comes about with $\Delta \mathrm{n}=0$ capture (Koster-Kronig transitions)-for example, the capture onto lithium-like iron in which the $2 \mathrm{~s}$ electron is excited to $2 \mathrm{p}$. This dielectronic rate is very large, and a proper calculation of it requires a careful calculation of the energy of each resonance and its autoionization width; large departures from the general formulae for dielectronic recombination rates are found. A schematic of the process is shown in Fig. IV-5. In beryllium-like iron the states 2 pnl with $n$ greater than about 11 are autoionizing. The spacing of states differing by one in $n$ is about $11 \mathrm{eV}$, so there are certain to be a few autoionizing levels within $10 \mathrm{eV}$ or so of the ionization threshold.

It would be quite interesting to produce a plasma with abundant lithium-like iron but with an electron temperature less than $50 \mathrm{eV}$, in order to study this process. The color temperature of the radiation would need to be a few hundred $\mathrm{eV}$ in order to produce this ion, which has an ionization potential of $2048 \mathrm{eV}$. We note that it 
is true that in accretion disks of $x$-ray binaries and Seyfert galaxies this ion of iron is probably found where the electron temperature is hotter, of order of a few hundred $\mathrm{eV}$ to $1 \mathrm{keV}$, so the near-threshold dielectronic recombination is relatively unimportant.

\section{Shock-Wave Ionized Media}

Another radiation transport study possible with the NIF involves shock waves in the target chamber to study photo-ionized nebulae. A wide range of experiments can be done to simulate the effect of energetic events in circumstellar and interstellar gases by utilizing a low-density gas fill in the 10-m-sized laser target chamber. The deposition of laser light onto a small target at the center of the chamber will lead to a bright UV and/or x-ray source (80-90\% of the laser light can be converted to $x$-rays). In a low-opacity gas a photo-ionized nebula will be created.

Detailed study of such processes will serve to benchmark models that are used in astrophysical contexts, such as quasars. Processes such as photoionization and excitation, radiative and dielectronic recombination, and line trapping would be studied. For a high-opacity gas, the $x$-rays will shortly be re-absorbed, leading to the creation of a shock wave in the chamber gas, similar to a supernova remnant in the interstellar medium. Similar work has already been done on a small scale at the Naval Research Laboratory. ${ }^{1}$ Fundamental studies of the propagation of such shocks, including effects of an applied magnetic field, can be done.

\section{Supernova Instabilities}

Hydrodynamic instabilities play a major role in determining the efficiency and performance of inertial confinement fusion implosions. In laserdriven implosions, high-performance capsules require high aspect ratios (the ratio of the radius to the shell thickness). These capsules are susceptible to hydrodynamic instabilities of the Rayleigh-Taylor, Richtmyer-Meshkov, and Kelvin-Helmholtz varieties, which can in principle severely degrade capsule performance. Recently the growth of the ablatively driven
Rayleigh-Taylor instability has been studied by indirect-drive experiments using a shaped $x$-ray drive pulse. ${ }^{2}$

The $x$-ray and gamma-ray observations of the famous supernova 1987A have indicated that radioactive cobalt is far more thoroughly distributed among the explosive debris in the envelope than was predicted by model calculations of thin-shell nucleosynthesis in the pre-supernova star. These observations of 1987A have strongly suggested the occurrence of largescale mixing in the ejecta during the explosion. 3,4 A larger scale mixing in the ejecta has also been suggested for Type Ib/Ic supernovae by light curve modeling and spectrum analysis.

The most promising mechanism for explaining mixing in the ejecta is a combination of the Rayleigh-Taylor and Kelvin-Helmholtz instabilities. The Rayleigh-Taylor instability can arise in the supernova envelope when the outwardly moving shock wave from the initial explosion propagates through layers of the star with radial stratification of the heavy elements. As the shock passes through the composition interfaces (i.e., oxygen/silicon, helium/carbontoxygen and hydrogen/helium), a rarefaction front moves back into the star, resulting in an effective reversal of gravity as low-density composition is pressure-accelerated into the underlying high-density composition.

Any perturbation at the interface (i.e., velocity perturbation or spatial perturbation) will get amplified by the Rayleigh-Taylor and Richtmyer-Meshkov instabilities and result in the overturning of light and heavy elements. This results in the mixing of heavy elements throughout the envelope of the supernova remnant, with associated observational consequences in the light curve.

A further mixing will occur as the dense "tongues" of the heavy elements experience differential shear with the lighter elements, resulting in Kelvin-Helmholtz instabilities. Thus the fingers of heavy and light fluid that developed initially get far more distorted and the mixing layer increases its width.

Eventually these instabilities become so nonlinear that the mixing layer appears to become fully turbulent. The properties of 
turbulently mixed layers may be equally important in understanding how interstellar clouds get reprocessed back into the interstellar medium. Efficient mixing of cloud and intercloud matter has been shown to occur after clouds get crushed by the interaction of strong shocks from supernova remnants. $5,6,7$

Experiments at the NIF would be ideally suited to detailed studies of this important astrophysical phenomenon. Densities and temperatures reached in the supernova-shocked envelope are well within the range attainable with the NIF.

The experimental techniques to study the development of Rayleigh-Taylor-induced mixing well into the nonlinear regime using streaked $x$-ray radiography of the unstable region have already been demonstrated by Remington et al. ${ }^{2}$ The NIF is ideally suited to producing the extremely strong shocks with Mach numbers $M \gg 1$ that occur in the supernova envelope. The shocks would be produced by shaped laser pulses ablating a foil or cylinder consisting of a sequence of material interfaces with density differences matching those in the supernova envelope. The shock wave would traverse the composite interfaces, which can be suitably perturbed, resulting in instabilities and mixing like those thought to occur in the supernova envelope. Shocks of sufficient strength are far out of the regime that is currently possible in standard shock-tube experiments, but will be relatively easy to achieve at the NIF.

Recent advances in high-resolution multidimensional hydrodynamics using adaptive mesh refinement (AMR) techniques now make it possible to accurately model the experiments with detailed three-dimensional simulations. Using newly developed AMR algorithms, ${ }^{8}$ recent calculations ${ }^{6,7}$ have studied the development of shock-induced Rayleigh-Taylor and RichtmyerMeshkov instabilities far into the nonlinear mixing regime. Calculations such as these, combined with NIF experiments, will allow a thorough study of the mixing in supernova explosions as well as studies pertaining to mixing in the interstellar medium.

Figure IV-6 (reproduced from Müller et al. ${ }^{9}$ ) shows the typical scenario for how RayleighTaylor mix enters into supernova modeling. The expanding inner core of nickel and silicon is being decelerated by the surrounding layer of lower density carbon and oxygen, which in turn is being decelerated by the outer layer of helium. At each interface one effectively has a heavy fluid "sitting on top of" a light fluid. Because of this, the interfaces are unstable to the Rayleigh-Taylor instability, and imperfections will grow. Typical growth factors are 100-1000.

Figure IV-7 shows how a similar situation could be set up on the NIF. Since the usual experiments focus the lasers into the hohlraum, the hohlraum in this case would be shot in an inside-out fashion. The drive lasers would impinge upon a thin (approximately $10-\mu \mathrm{m}$ ) cylindrical nickel hohlraum with sinusoidal perturbations imposed on the outer surface. The outer surface of nickel would be coated with $\approx 100 \mu \mathrm{m}$ plastic, and the whole package would reside in a $\approx 10$-atm fill of helium.

As the drive turns on, a strong shock ( $\approx 100 \mathrm{Mbar}$ ) would be launched radially

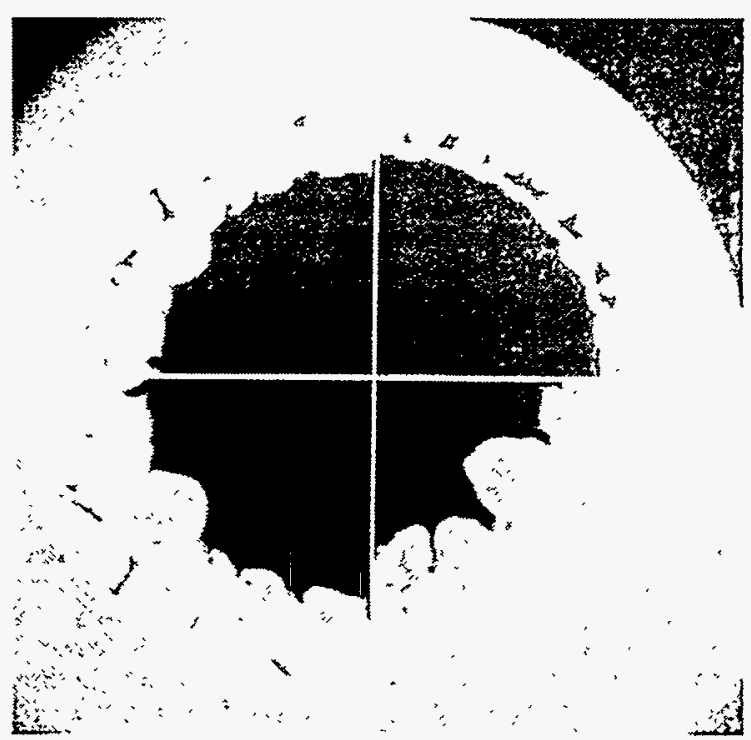

Figure IV-6. Rayleigh-Taylor mix in supernova modeling. The four quadrants show contours of total density times the mass fraction of $4 \mathrm{He}$ at four instants during a supernova explosion. The four times proceed counterclockwise beginning with the upper right section. Note that the original sphere of helium is already breaking up in the first instant shown. 
outwards through the various layers. Each interface would be unstable to the RichtmyerMeshkov instability, and perturbation growth would begin. Then as the hohlraum explodes radially outwards, the nickel core would be decelerated by the lower density plastic layer, which in turn would be decelerated by the helium fill, in much the same fashion as for the supernova shown in Fig. IV-6.
Diagnosis would be from face-on, edge-on, and end-on radiography, in the standard fashion currently utilized on Nova. A large-area copper backlighter, with $\mathrm{K} \alpha$ emission at $8.4 \mathrm{keV}$, might be suitable. If this "direct-drive" approach to accelerating the nickel hohlraum proves to imprint an intolerable level of non-uniformity on the nickel-plastic interface, a hybrid indirectdrive approach would be the fall-back. Here,

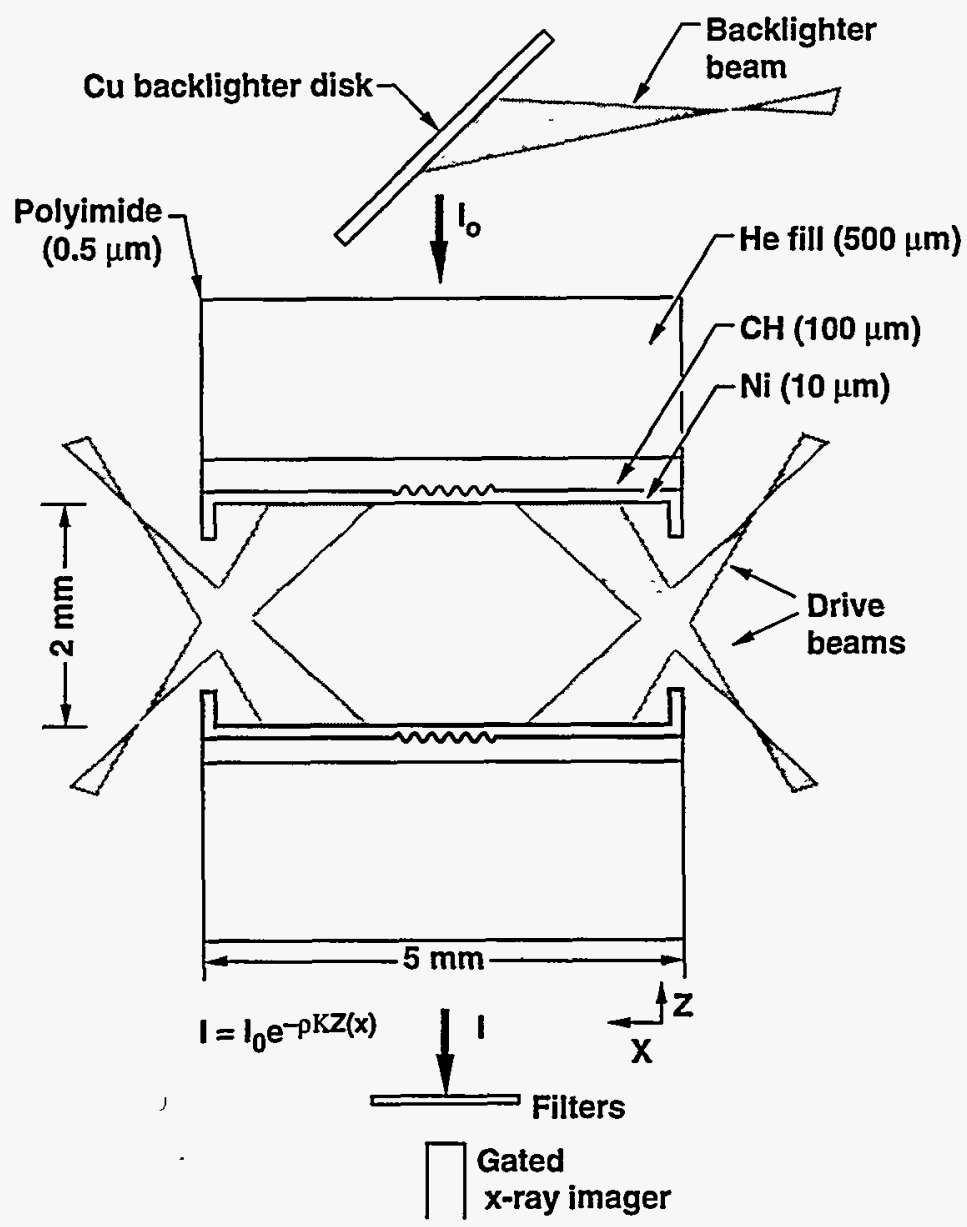

Figure IV-7. Schematic setup of an astrophysical mix experiment to mimic the mixing in a supernova explosion. The drive lasers would impinge upon a thin (approximately $10-\mu \mathrm{m}$ ) cylindrical nickel hohlraum with sinusoidal perturbations imposed on the outer surface. The outer surface of nickel would be coated with $\approx 100 \mu \mathrm{m}$ plastic, and the whole assembly would reside in a $\approx 10$-atm fill of helium. As the drive turns on, a strong shock $(\approx 100 \mathrm{Mbar})$ would be launched radially outwards through the various layers. Each interface would be unstable to the Richtmyer-Meshkov instability, and perturbation growth would begin. Then as the hohlraum explodes radially outwards, the nickel core would be decelerated by the lower density plastic layer, which in turn would be decelerated by the helium fill, in much the same fashion as $\alpha$ in a supernova. Diagnosis would be from face-on, edgeon, and end-on radiography. 
the lasers would hit an inner solid high- $Z$ cylindrical rod, generating an $x$-ray drive. The $x$-ray drive would in turn drive the nickel hohlraum outwards.

Note that already on Nova with $\mathrm{a} \approx 20-30-\mathrm{kJ}$ drive, growth factors of nearly 100,10 shock strengths of $\approx 50 \mathrm{Mbar}$, and Mach numbers of $\approx 35$ have been achieved. ${ }^{11}$ On the NIF, with 1.8 MJ of drive energy, achieving growth factors of 100-1000 with 50- to 100-Mbar shocks, as suggested by the supernova modeling, should be straightforward.

\section{E. High-Velocity Cratering}

One of the interesting issues in lunar and planetary physics is that of crater formationsurface damage-by high-velocity impacts. The velocities of projectiles in the solar system range up to $50 \mathrm{~km} \mathrm{~s}^{-1}$ (for projectiles in retrograde orbits), which is out of the range for gas guns. This kind of high-velocity projectile impact study can easily be done using the laser-driven flyer plate technology possible on the NIF, as discussed in the Hydrodynamics and Material Properties sections. The physics issues to be studied include the systematics of crater dimensions with projectile shape and size-the nature of the damage mechanism. An example would be the dependence of the cratering on the type of material forming the target.

A further set of issues is related to electromagnetic effects caused by the impact. It is known that electromagnetic noise is produced at the time of impact. It is also found that there are substantial magnetic anomalies in the rock underlying lunar impact craters. The mechanisms of these phenomena can be studied by performing the flyer plate experiments with weakly magnetized targets.

The suitability of this project to the NIF arises from the ease of attaining the high impact velocity and the availability of larger projectile sizes. On Nova the projectiles with a suitably large velocity are so small that edge effects obscure the phenomena to be studied.

\section{F. Thermonuclear Reaction Rates in Stars}

Although nuclear reaction rates have been the subject of intense studies for decades, it remains true that few direct experimental studies have been made at the actual energies at which reactions occur in stellar environments. Figure V-1 summarizes a number of astrophysical thermonuclear environments and their associated temperatures. The actual energies relevant for the nuclear cross-section $s$ are given by the so called Gamow window, which represents the interplay between the Maxwellian tail of the reactant velocity distribution and the rapidly diminishing reaction cross-section at energies below the Coulomb barrier.

The relative merits of the NIF for obtaining information on nuclear reaction rates, as opposed to the more usual accelerator technique, can be determined as follows. Consider the problem count rate. For a conventional accelerator experiment, the reaction rate for projectile $a$ plus target nucleus $b$ to produce some product nucleus $c$ can be written:

$$
r(a+b, \text { Accelerator })=I_{a} \sigma_{a b} n_{b} \Delta x,
$$

where $I_{a}$ is the incident projectile current, $\sigma_{a b}$ is the cross-section for the reaction between projectile $a$ and target nucleus $b$, while $n_{b}$ and $\Delta x$ are the target density and thickness, respectively. Consider a typical reaction, the ${ }^{12} \mathrm{C}(\mathrm{p}, \gamma)^{13} \mathrm{~N}$ reaction, which plays a role in the CNO cycle. The hydrogen burning temperatures for this reaction are from about 1 to about $3 \mathrm{keV}$. At $3 \mathrm{keV}$, the Gamow window for this reaction is at approximately $40 \mathrm{keV}$, for which one expects a cross-section of order 0.01 picobarn. 12

For optimistic accelerator conditions with an incident current of $100 \mu \mathrm{A}$ on a carbon target of density $10^{23} \mathrm{~cm}^{-3}$ and a thickness of $10^{-4} \mathrm{~cm}$ (the approximate range of protons in carbon for this energy), the reaction rate would be at most five events per day, quite difficult to pick out of the cosmic ray background.

On the other hand, the reaction rate in a thermalized NIF capsule can be written:

$$
r(a+b, N I F)=\langle\sigma v\rangle N_{a} n_{b}
$$


where $\langle\sigma v\rangle$ is the Maxwellian-averaged product of cross-section times relative velocity, $n_{b}$ is the density of nucleus $b$, and $N_{a}$ is the total number of nuclear species $a$ that can react with $b$. At the temperature of $8 \mathrm{keV}$ that might be attained in a capsule imploded using the NIF, the expected Maxwellian averaged reaction rate ${ }^{13}$ would be $N_{a}<\sigma v>10^{-5} \mathrm{~cm}^{3} \mathrm{~mole}^{-1} \mathrm{~s}^{-1}$. At a hydrogen density of $10^{2} \mathrm{~g} \mathrm{~cm}^{-3}$, reacting with $10^{17}$ carbon atoms $(2 \mu \mathrm{g})$ for $\approx 100 \mathrm{ps}, \approx 10^{4}$ reactions would be expected in a single event. This number of radioactive ${ }^{13} \mathrm{~N}$ nuclei would have a half-life $t_{1 / 2}$ of $\approx 10 \mathrm{~min}$, so it would be possible to count them after an event. Alternatively, the pulse of $\approx 2-\mathrm{MeV}$ gamma rays might be detected with scintillators positioned around the target. The fact that the events are produced all at once avoids the usual low signal-to-noise ratio associated with conventional accelerator experiments, in which the reactions occur one at a time and are therefore difficult to distinguish from ambient room background.

Of course, such experiments would not be without their own difficulties. The various state variables are changing rapidly and it is necessary to have accurate diagnostics and simulations of the thermodynamic evolution of an event, including departures from equilibrium. The reaction rates are so sensitive to temperature that it will be difficult to know the burn temperature to sufficient accuracy. It is possible to envision, however, a relative measurement of several species at once, making it possible to fix the temperature with the most sensitive reaction.

Also, these are explosive environments not easily amenable to conventional nuclear counting techniques. Backgrounds are also certain to develop. Nevertheless, it is possible to develop a class of nuclear experiments in which a catcher blanket that could collect radioactive products would be placed at some distance from the capsule explosion. The catcher material could later be removed from the chamber and counted for accumulated radioactivity. The final step would be to compare the nuclear yields produced in the experiment with the yields predicted by applying thermonuclear network codes to the conditions one believes occurred in the event. This comparison could be used to test both the codes and the reliability of the thermonuclear reaction rate data. It could also aid as a diagnostic of the capsule history, because the thermonuclear reaction rates are quite temperature sensitive.

Interesting nuclear reactions to study besides ${ }^{12} \mathrm{C}(\mathrm{p}, \gamma){ }^{13} \mathrm{~N}$ would be ${ }^{3} \mathrm{He}\left({ }^{3} \mathrm{He}, 2 \mathrm{p}\right){ }^{4} \mathrm{He}$, and ${ }^{3} \mathrm{He}\left({ }^{4} \mathrm{He}, \gamma\right){ }^{7} \mathrm{Be}$. These latter two are part of the proton-proton chain of hydrogen-burning reactions in solar-type stars, while the first is part of the CNO cycle. The competition between the proton-proton reaction and CNO cycle for the destruction of ${ }^{3} \mathrm{He}$ determines how much ${ }^{7} \mathrm{Be}$ is produced (and ultimately how much ${ }^{8} \mathrm{~B}$ ), and therefore how great the flux of neutrinos should be, as detected in the Homestake Mine solar neutrino experiment.

The reaction rates that take place in the CNO bi-cycle are illustrated in Fig. IV-8. The primary loop is shown as the outer loop, the secondary loop is shown within. In both loops, the energy generation is the energy gained by creating the $\mathrm{He}^{4}$ from the four $\mathrm{H}^{1}$, with the residual loss of the two neutrinos, $v$, divided by the time it takes to circle the cycle. The times for the radiative decays of the $\mathrm{N}^{13}, \mathrm{O}^{15}$, and $\mathrm{F}^{17}$ are fixed but the remainder of the rates will depend on the temperature and density of the constituents. The densities accessible to the NIF will permit the critical determination of these rates in regimes relevant to astrophysical situations.

This branching ratio continues to be a significant uncertainty in the interpretation of the solar neutrino experiment, and a refinement of our knowledge here would be of great value. The particle decay rate of the ${ }^{3} \mathrm{He}\left({ }^{3} \mathrm{He}, 2 \mathrm{p}\right){ }^{4} \mathrm{He}$ reaction is some $10^{5}$ times greater than that of the radiative decay rates of ${ }^{12} \mathrm{C}(\mathrm{p}, \gamma)^{13} \mathrm{~N}$ and ${ }^{3} \mathrm{He}\left({ }^{4} \mathrm{He}, \gamma\right){ }^{7} \mathrm{Be}$. In order to diagnose ${ }^{3} \mathrm{He}\left({ }^{3} \mathrm{He}, 2 \mathrm{p}\right)$ ${ }^{4} \mathrm{He}$ it will be necessary to measure the activation produced by the $\approx 5-\mathrm{MeV}$ protons in a witness foil placed near the target, or in the wall of the hohlraum itself.

Figure IV-1 showed the way in which NIF conditions overlap various stellar burning phases. Temperatures up to carbon-burning are achievable with ignition. Even with the driver alone (i.e., without ignition), hydrogen burning and helium burning temperatures are obtainable, 


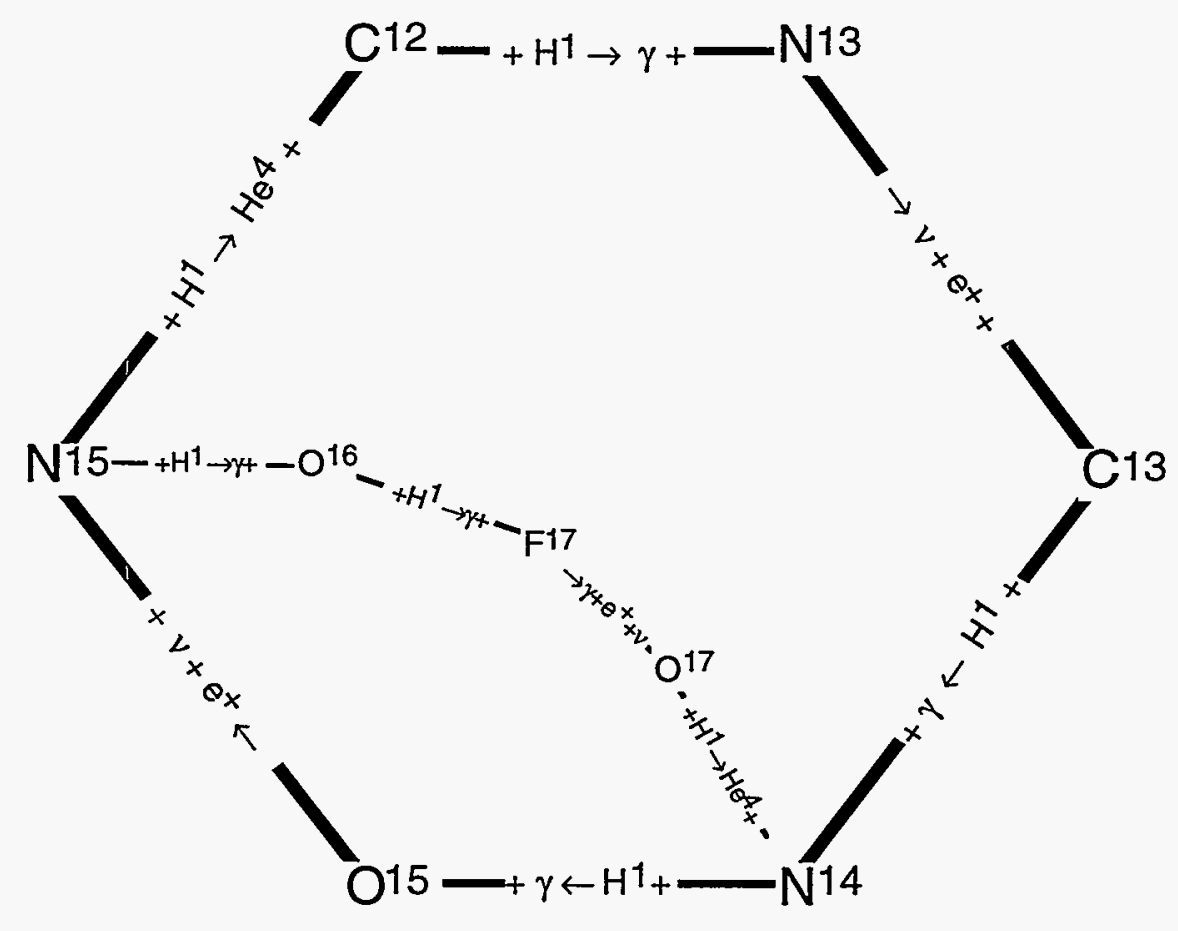

Figure IV-8. Reaction rates that form the CNO bi-cycle. The primary cycle is shown as the outer loop, cycling around $\mathrm{C}^{12}, \mathrm{~N}^{13}, \mathrm{C}^{13}, \mathrm{~N}^{14}, \mathrm{O}^{15}$, and $\mathrm{N}^{15}$. The secondary loop connects the $\mathrm{N}^{15}, \mathrm{O}^{16}, \mathrm{~F}^{17}$, and $\mathrm{O}^{17}$. In both loops the energy generation is the energy gained by creating the $\mathrm{He}^{4}$ from the four $\mathrm{H}^{1}$, with the residual loss of the two neutrinos, $v$, divided by the time it takes to circle the cycle.

as are the density regimes for both hydrogen and helium burning.

Figure IV-9 shows the evolution of the early universe through the big bang. Lines are drawn for both baryons and electrons for baryon densities corresponding to both 0.01 and 1.0 times the present closure density of the universe $\Omega_{b}$. It seems that with an appropriate capsule design, it would be possible to nearly reproduce various conditions in the early universe during much of the critical epoch (first three minutes) of primordial nucleosynthesis.

\section{G. Electron-Positron Plasmas}

Thermally produced electron-positron plasmas are thought to play an important role in the evolution of the cores of massive stars, neutron-star and black-hole accretion disks, pulsars, quasars, astrophysical gamma-ray bursters, and in the big bang. Although it is not likely that the NIF could be used to produce a pair plasma with the currently proposed design parameters, it is possible that some pairs will be produced in a way that is similar to the formation of pairs in such astrophysical environments. Hence, experiments with the NIF may shed light on pair-plasma formation and radiation transport issues. We therefore include here a brief discussion of some of the physics issues regarding pair production.

In the last few years, discoveries of intense broadened 511-keV annihilation lines from several galactic black-hole candidates suggest that in addition to transient-pair production, steady-state thermal pair plasmas may exist. Since pairs annihilate on short time scales, maintaining such steady-state conditions requires the copious production of pairs, in order to balance the annihilation rate. Such pair-balanced steady plasmas represent a new state of matter with unique radiative and thermodynamic properties different from ordinary plasmas.

For a plasma to be in a steady state, the heating rate must be balanced by the cooling rate, 


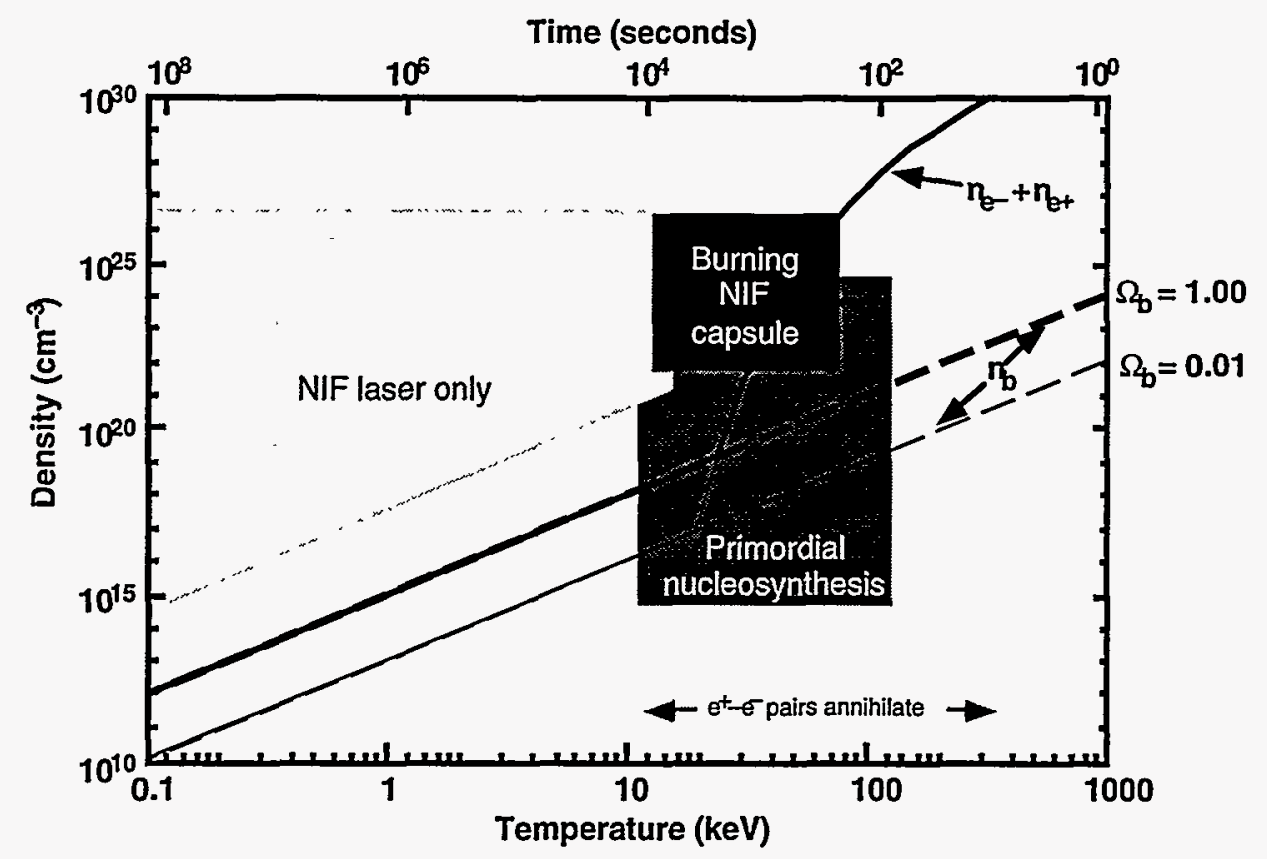

Figure IV-9. Trajectories in temperature and density parameter space for some big-bang nucleosynthesis models. The boxes indicate the overlap between cosmological models and plasmas the NIF can produce. The models are for two different baryon densities. The thick lines indicate the present baryon density of the universe that would be needed to close the universe, while the thin lines indicate a model with 0.01 of the critical density to close the universe. The dashed lines are the baryon density and the solid lines are the sum of the electron and positron densities. The upper abscissa is the time from the big bang. Note that the baryon curves start at the point of "weak freezeout" at $1 \mathrm{MeV}$, below which the weak interaction processes depart from equilibrium and the neutron-proton ratio becomes locked in.

which consists of bremsstrahlung, inverse Compton scattering, and pair annihilation. It turns out that for a pair-balanced plasma there exists a fundamental limit to the temperature of $\approx 10 \mathrm{MeV}$ for hydrogen, above which pair creation can no longer be balanced by annihilation and pair density will exponentiate rapidly, leading to a pair-dominated plasma and net cooling of the system. This limiting temperature, referred to as the BKZS limit, is much too high for NIF-based experiments; however, the limiting temperature can be lower for a high- $Z$ or high magnetic-field plasma.

The laser intensities available at the NIF may permit the formation of some pairs out of the suprathermal electrons during a hightemperature deuterium-tritium (DT) burn. In equilibrium, at a temperature of $50 \mathrm{keV}$, the vacuum pair abundance is suppressed by only a factor of $\exp \left(-m_{e} c^{2} / k T\right) \approx 10^{-5}$, implying a pair density as high as $\approx 10^{21} \mathrm{~cm}^{-3}$. This factor will be reduced, however, by the ratio of the burn time (of order $\approx 10 \mathrm{ps}$ ) to the equilibration time. The equilibration time is of order of the time scale for pair creation.

The most promising avenue to the production of a pair plasma seems at this time to be to use the petawatt laser under development as an adjunct to Nova. One of the Nova beams is intercepted partway through the amplification chain and is subjected to pulse compression, producing a pulse of $1.06-\mu \mathrm{m}$ light containing about $1 \mathrm{~kJ}$ of energy in a pulse length of $1 \mathrm{ps}$. If this can be focused to a spot size limited only by diffraction, then an intensity of $10^{21} \mathrm{~W} / \mathrm{cm}^{2}$ can be produced. The paper by Wilks et al. ${ }^{1}$ shows how such a laser pulse can produce relativistic electrons and confine them through a combination of the optical fields and the ponderomotive force. 
At very high laser intensities $I$, satisfying I $\lambda_{\mu}{ }^{2}>1.4 \times 10^{18} \mathrm{~W} \mathrm{~cm}^{-2} \mu \mathrm{m}^{2}$ where $\lambda_{\mu}$ is the laser wavelength in microns, the electron jitter momentum becomes relativistic and scales roughly as the square root of the intensity. If the jitter energy $E$ of the electrons is larger than $2 m c^{2}$, as it is for I $\lambda_{\mu}^{2} \gg 1.4 \times 10^{18}$, then collisions of the electrons with ions of charge $Z$ in the plasma will produce pairs; the cross-section for this process is of order $10^{-30} \mathrm{Z}^{2} \mathrm{~cm}^{2}$, weakly dependent on electron energy in this range. This would be sufficient in a critical-density gold plasma to produce in $1 \mathrm{~ns}$ a pair density of order $10^{-5}-10^{-4}$ times that of the electrons.

This fraction would be increased if a density substantially above the critical density could be used, as is suggested by Wilks et al. ${ }^{14}$ It may be important in the experiment design to provide laser illumination from opposing directions; this allows the pairs that are created to be trapped by the optical field and be themselves accelerated, producing more pairs in turn. The pairs would annihilate over a time likely to be longer than the laser pulse, so the result would be a temporally broadened annihilation line in the gamma-ray spectrum. The line profile plus the Comptonized bremsstrahlung continuous spectrum will form the most useful diagnostics of the pair plasma (e.g., Liang and Dermer ${ }^{15}$ ).

An extension of the peta-watt concept to the $\mathrm{NIF}$-providing compressed pulses and diffraction-limited focusing capability on some number of the NIF beams-would extend the spatial and temporal scales for this relativistic plasma, and perhaps make creation of a pairdominated plasma a reality.

\section{H. References}

1. B. H. Ripin, A.W. Ali, H. R. Griem, J. Grun, S. T. Kacenjar, C. K. Manka, E. A. McLean, A. N. Mostovych, S. P. Obenschein, and J. A. Stamper, in Laser Interactions and Related Plasma Phenomena 7, G. Miley and H. Hora, Eds. (Plenum Press, New York, 1986), p. 857.

2. B. A. Remington, S. V. Weber, S. W. Haan, J. D. Kilkenny, G. G. Glendinning, and R. J. Wallace, Phys. Fluids B 5, 2589 (1993).
3. Kumagai T. Shigeyama, K. Nomoto, M. Itoh, J. Nishimura, and S. Tsurata, Ap. J. 345, 412 (1989).

4. D. Arnett, J. Bahcall, R. Kirschner, and S. Woosley, Ann. Rev. Astron. Astrophys. 27, 629 (1989).

5. R. I. Klein, C. F. McKee, and P. Colella in The Evolution of Interstellar Medium, L. Blitz, Ed., Astronomical Society of the Pacific Conference Series 12, 117 (1990).

6. R. I. Klein, C. F. McKee, and P. Colella, Ap. J. 420, 213 (1994).

7. R. I. Klein, J. Bell, R. Pember, and T. Kelleher in the proceedings of the 4th International Conference on Compressible Turbulence, Cambridge, UK (in press).

8. J. Bell, M. Berger, J. Saltzman, and M. Welcome, SIAM J. Sci. Comp. 15, 127 (1994).

9. E. Müller, B. Fryxell, and D. Arnett, Astron. Astrophys. 251, 505 (1991).

10. B. A. Remington, S. W. Haan, G. G. Glendinning, J. D. Kilkenny, D. H. Munro, and R. J. Wallace, Phys. Rev. Lett. 67, 3259 (1991), and B. A. Remington, S. W. Haan, G. G. Glendinning, J. D. Kilkenny, D. H. Munro, and R. J. Wallace, Phys. Fluids B 4, 967 (1992).

11. B. A. Hammel, D. Griswold, O. L. Landen, T. S. Perry, B. A. Remington, P. Miller, T. A. Peyser, J. D. Kilkenny, Phys. Fluids B 5, 2259 (1993); B. A. Hammel, J. D. Kilkenny, D. H. Munro, B. A. Remington, H. N. Kornblum, T. S. Perry, D. W. Phillion, and R. J. Wallace, Phys. Plasmas 1, (1994-in press); and G. Dimonte and B. A. Remington, Phys. Rev. Lett. 70, 1806 (1993).

12. D. D. Clayton, Principles of Stellar Evolution and Nucleosynthesis (McGraw-Hill, New York, 1968).

13. M. Harris, W. Fowler, G. Caughlann, and B. Zimmerman, Ann. Rev. Astron. Ap. 21, 165 (1983).

14. S. C. Wilks, W. L. Kruer, M. Tabak, and A. B. Langdon, Phys. Rev. Lett. 69, 21383 (1992).

15. E. P. Liang and C. D. Dermer, Ap. J. 325, L39 (1988). 


\section{Section V}

\section{Hydrodynamics}

\section{A. Techniques}

Experience gained by researchers on the existing high-energy lasers has demonstrated that a variety of fluid-dynamics experiments may be carried out on such facilities. In particular, two types of experiments have emerged as the leading hydrodynamic experimental approaches-those that involve radiography and others that utilize self-emission.

To provide enough background to understand the belief that the high-energy lasers provide a vehicle to open novel areas of hydrodynamics not possible at other facilities, we provide the following illustrations of technique, which show clearly the current limitations as well as the advanced state of the techniques developed. These particular examples are selected from work conducted on Nova, because many of their features are shared with other experiments and thus they permit some general observations to be drawn.

\section{Radiography-A Typical Configuration}

In a series of experiments, we can investigate the quasi-two-dimensional flow of a jet formed by the passage of a shock over a hemispherical protrusion on a density interface. A side view of the configuration for these experiments, shown end-on to the hohlraum, is depicted in Fig. V-I.

The experimental package- the object to be observed-consists of a beryllium tube with an insert of bromine-doped plastic joined to carbon foam, two materials with different densities. The inner diameter of the beryllium tube is $700 \mu \mathrm{m}$, and its length is $2000 \mu \mathrm{m}$. There is a hemispherical feature at the interface between the bromine-doped plastic and the carbon foam, to seed the instability.The hemispherical feature is produced by using a lathe to machine a hemispherical hole in a bromine-doped plastic cylinder and a matching hemispherical bump (which has a radius of $150 \mu \mathrm{m}$ ). A photograph of the carbon part is shown in Fig. V-2.

The tube is abutted to the center of the exterior of a gold hohlraum, which provides the $x$-ray drive. A hole exposes the end of the package to the hohlraum interior. A large-area titanium $x$-ray backlighter provides backillumination for an $x$-ray framing camera, which images the materials during transmission of the shock wave.

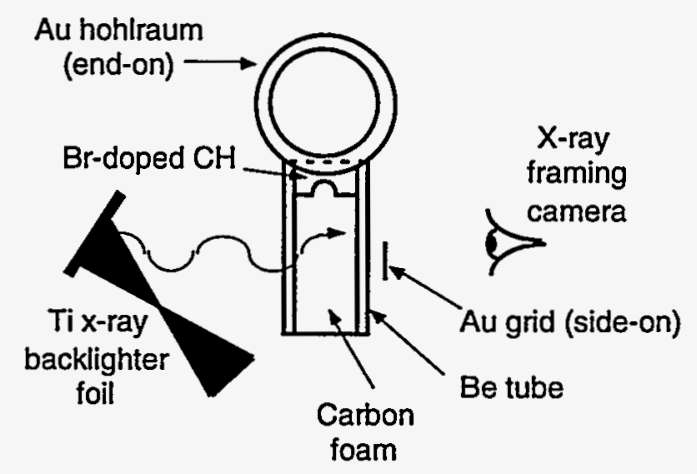

Figure V-1. Side view of the jet experimental arrangement, shown end-on to the hohlraum. The experimental package (target) is abutted to a gold hohlraum. The target is composed of a beryllium tube in which bromine-doped plastic is joined to a carbon foam cylinder with a hemispherical feature on the interface. A largearea titanium $x$-ray backlighter provides backillumination for an $x$-ray framing camera, which images the materials in transmission. 

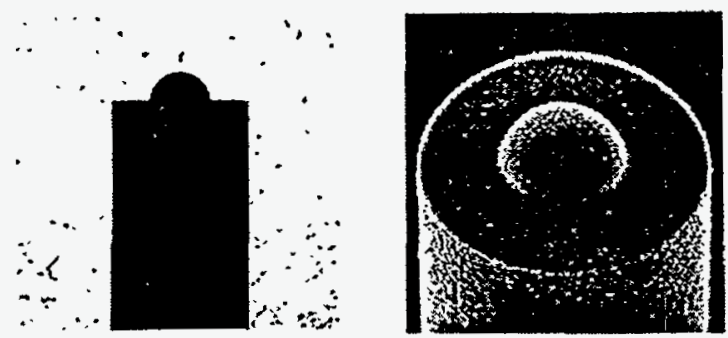

Figure V-2. Microscope photographs of the cylindrical carbon foam piece, showing the diamond-turned hemispherical feature. The hemisphere radius is $150 \mu \mathrm{m}$.

The temporal sequence of events in the experiment is as follows. Eight of the Nova laser beams are focused into the hohlraum, which heats up rapidly to a temperature of 220 to $240 \mathrm{eV}$. The bromine-doped plastic end of the experimental package, exposed to the interior of the hohlraum, rapidly ablates. The ablation causes a strong shock wave to be launched into the plastic. The shock travels outward through the experimental package, and encounters the density interface between the plastic and the carbon foam. Upon crossing the interface, the shock-induced acceleration produces an instability between the two different-density materials, seeded by the 150- $\mu \mathrm{m}$-radius hemisphere.

Shortly before the time chosen to image the flow, the ninth and/or tenth Nova beams are used to illuminate an $\mathrm{x}$-ray backlighter foil. Titanium and iron are typically used as foil materials; they produce 4.7 - and $6.7-\mathrm{keV} x$-rays, respectively.

The $x$-rays are used to radiograph the experimental package sideways, using either a two-dimensional framing camera or an x-ray streak camera. The brominated plastic is more opaque to the backlighter $x$-rays than the undoped plastic, so it appears darker in the images. Shocked, or compressed, material also appears darker because of its larger density.

When the opacities of the various components are known, the transmission can be unfolded to provide material distributions. This knowledge of the opacity is central, as it is one of the fundamental limitations of the experimental technique for providing quantitative data. The diagnostic technique is discussed in more detail in Hammel et al. ${ }^{1}$

The passage of the shock from the plastic ablator section over this "bump" generates the jet by the same mechanism as the RichtmyerMeshkov instability. As the jet propagates, it experiences shear on its edges, leading to a Kelvin-Helmholtz roll-up.

Experiments were conducted that imaged the jet at $19.5 \mathrm{~ns}$ and $25.8 \mathrm{~ns}$ after the initiation of the drive beams. Comparison of the data and simulated radiographs from two-dimensional hydrodynamic simulation are shown in Figs. V-3 (for $19.5 \mathrm{~ns}$ ) and V-4 (for $25.8 \mathrm{~ns}$ ). The data quality is limited by several factors, including the small size of the jet, the short gating time of the available detector, and the availability of only a single backlighter beam at late time (that is, at a time greater than $8 \mathrm{~ns}$ ), when these data were acquired. The picture at $25.8 \mathrm{~ns}$ is among data with the longest time delay between firing the main laser and taking an image that was ever carried out on Nova.

The comparison between the data (left) and the calculation (right) for early time is shown in Fig. V-3. The data shows two dark bands, associated with the swirls, of similar size and location as the features in the simulation. The comparison at the later time shown in Fig. V-4 is not as striking. The experimental picture shows a single, wide, dark band below a large U-shaped feature. The calculation displays much more structure, and is a little narrower and more advanced down the tube. The effect of the shocks can be seen in the simulations, but not in the data. It is noted that the comparison of "swirls" and other gross features in the experiments is not commensurate with the need to obtain quantitative data on the underlying physical processes involved in the hydrodynamics. However, the development of these techniques, which are extremely complex, can be undertaken in preparation for future quantitative work.

Figures V-3 and V-4 show that radiography is quite advanced, but also (and most important) that there is a real need for the advantages to be accrued from the NIF, as these will allow much 

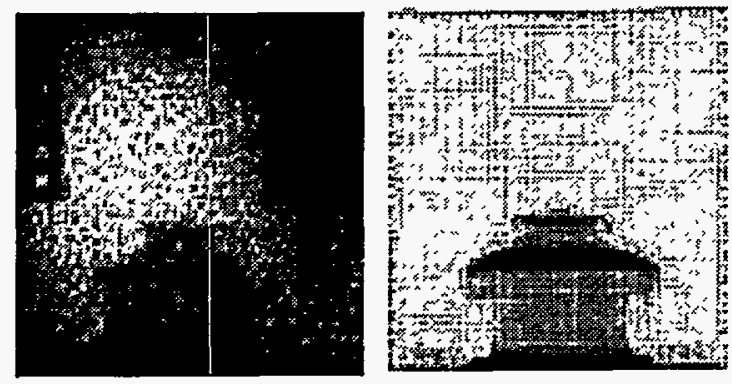

Figure V-3. Comparison of experimental data with simulation at $t=19.5$ ns (early time) for the jet experiment shown in Figs. V-1 and V-2. On the left is the $x$-ray image showing the evolving jet. On the right is the simulated $x$-radiograph showing the features of the jet. The comparison shows relatively good agreement between the actual data and the simulation.

better data to be obtained. The relaxation of size limitations, the duration of drive pulses, and the use of numerous beams for backlighting, all to be available on the NIF, will make these types of experiments more realistic.

\section{Shock Planarity}

To investigate possible methods for making shocks planar for future studies, a number of hohlraum/package variations have been tested. These illustrate the flexibility of the high-energy laser experiments and also some of the limitations of the current methods.

An experimental package was constructed for the shock planarity experiments. The package used a thickness of 150 or $300 \mu \mathrm{m}$. Eight beams of the laser were focused onto the inside of a gold hohlraum to create an $x$-ray drive. That drive impinged on the breakout target, and an optical streak camera was used to view the package from its end, rather than its side. With this scheme, the absolute time of the break and the uniformity of the shock that traverses the target can be measured. See Fig. V-5 for a schematic of the experiment.

The principle of the experiment is that shock breakout through the rear surface of the package is accompanied by heating, and the resulting radiation is detected by the optical streak camera. If the shock is curved with the central portion
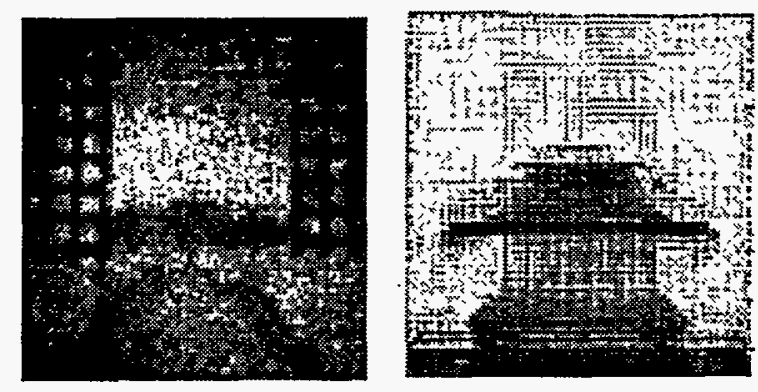

Figure V-4. Comparison of experimental data with simulation at $\mathbf{t}=\mathbf{2 5 . 8} \mathrm{ns}$ (late time) for the jet experiment shown in Figs. V-1 and V-2. On the left is the $x$-ray image showing the evolving jet. On the right is the simulated $x$-radiograph showing the features of the jet. The comparison shows agreement that is not as striking as for earlier time (Fig. V-3).

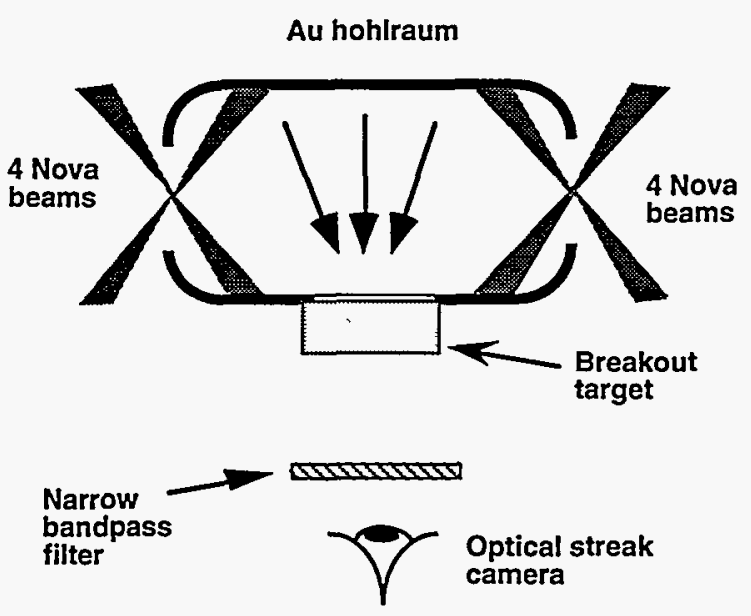

Figure V-5. Schematic of a shock breakout experiment to measure the planarity of a shock front. Eight beams of the laser are focused onto the inside of a gold hohlraum to create an x-ray drive. That drive impinges on the breakout target, which is imaged onto a streaked optical pyrometer (streak camera), viewing the package from its end rather than its side.

leading when it reaches the rear surface, the breakout occurs first at the center then proceeds radially as the rest of the shock front reaches the surface. When the optical streak camera is used to image a diameter of the rear surface, the resulting streaked image of a curved shock is also curved. 
Two of the candidate mounting schemes tested to determine the effects of mounting on the planarity of a shock are shown in Figs. V-6, and their corresponding data from the streaked optical pyrometer are shown in Fig. V-7. One scheme, the standard scheme, consists of simply abutting the package against the flat of the hohlraum, over the hole. The second is a version of a design that is called a "tophat" because of its appearance. The standard scheme will be shadowed by the hohlraum wall, whereas the tophat mount provides for a uniform illumination of the target.

It is clear from the data (Fig. V-7) that the breakout in the standard mount is not flat-the breakout takes over $1.7 \mathrm{~ns}$ from first arrival to the edges of the package. The tophat mount, on the other hand, has a very flat shock that breaks out over the entire diameter in 400 ps, with breakout over the central $80 \%$ occurring in only 100 ps. Note that the central region, on the position scale between 0.2 and 0.6 , represents the breakout data, while the bright sidebands are artifacts of the experiments, not related to the shock breakout.

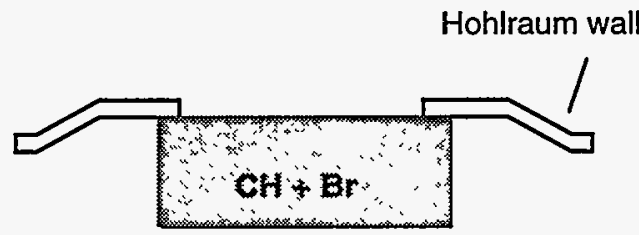

Standard mounting scheme

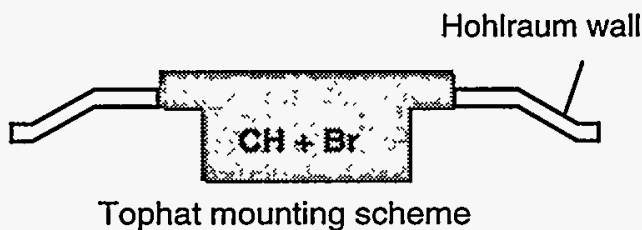

Figure V-6. Two candidate mounting schemes to study planarity of the shock generated by $x$-ray drive impinging on the brominated plastic from above. The upper scheme is the standard scheme, where the package is simply abutted against the flat of the hohlraum, over the hole. The second scheme is called a "tophat" mount because of its appearance.
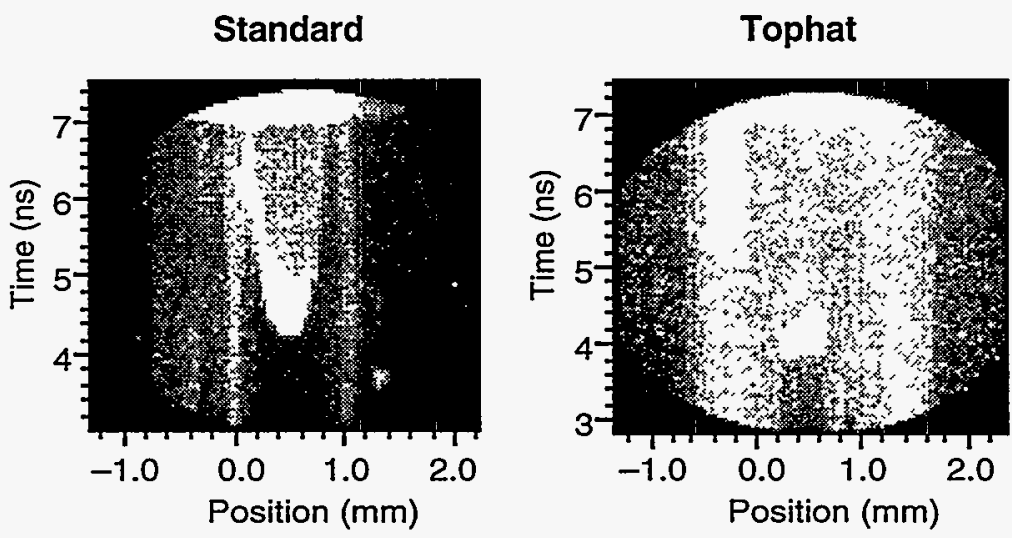

Figure V-7. Breakout data for the two different mounting schemes, standard and tophat, shown in Fig. $V-6$. Note that the central region, on the position scale between 0.2 and 0.6 , represents the breakout data, while the bright sidebands are artifacts of the experiments, not related to the shock breakout. The breakout in the standard mount is not flat-the breakout takes over $1.7 \mathrm{~ns}$ from first arrival to the edges of the package. The tophat mount, on the other hand, has a very flat shock that breaks out over the entire diameter in 400 ps, with breakout over the central $80 \%$ occurring in only 100 ps. 


\section{B. Stable Hydrodynamics}

\section{Planar Geometry}

A series of experiments has been performed to investigate the hydrodynamic motion of plasmas. The goal is to design an experiment where the hydrodynamics could be rigorously investigated without other complicating factors. In particular, the desire is to avoid any problems arising from laser-plasma interactions or strength-of-material complications. Therefore, it is considered essential to deposit the initial energy in a well-defined manner. These considerations led to the use of a short pulse of penetrating $x$-rays to provide the initial heating for the samples.

The $x$-rays were produced by focusing one or more of the beams of Nova onto a thin gold foil approximately $2000 \AA$ thick. The laser pulse length was 1 ns long. It was found that about $10 \%$ of incident laser light was converted into gold $\mathrm{M}$-band X-rays with a photon energy between 2 and $3 \mathrm{keV}$ (see Section VIII, Radiation Sources, for more information on the $x$-ray sources).

To filter out $x$-rays of lower photon energy (radiation below the critical density for solid matter), a $50-\mu \mathrm{m}$-thick plastic foil was placed at a distance of $200 \mu \mathrm{m}$ from the gold foil between the gold $x$-ray converter and the sample to be heated. We placed a 3- $\mu \mathrm{m}$ aluminum foil at a distance of $1000 \mu \mathrm{m}$ away from the gold foil. This foil absorbed approximately half of the incident gold $M$-band radiation.

The heating spectrum has been accurately characterized using the $x$-ray filtered diodes instrument at Nova. This is a ten-channel detector that uses absolutely calibrated filtered $\mathrm{x}$-ray diodes. $\mathrm{X}$-ray crystal spectrometers were also used to further spectrally characterize the radiation. With this arrangement an aluminum foil was heated to a few eV. At these temperatures the $\mathrm{x}$-ray absorption coefficients of the aluminum are very well known and energy deposition in the aluminum is well characterized. The schematic layout of these experiments is shown in Fig. V-8.

The expansion of the aluminum foil was observed by using a time-gated $x$-ray imager. A $2000-\mu \mathrm{m}$ tantalum foil was placed perpendicular to the direction of the motion at a distance of $\sim 1 \mathrm{~cm}$ from the aluminum foil, thus allowing observation across the foil. The tantalum was illuminated by one of the Nova laser beams, which had been smoothed by a random phase plate, and this produced a uniform $x$-ray

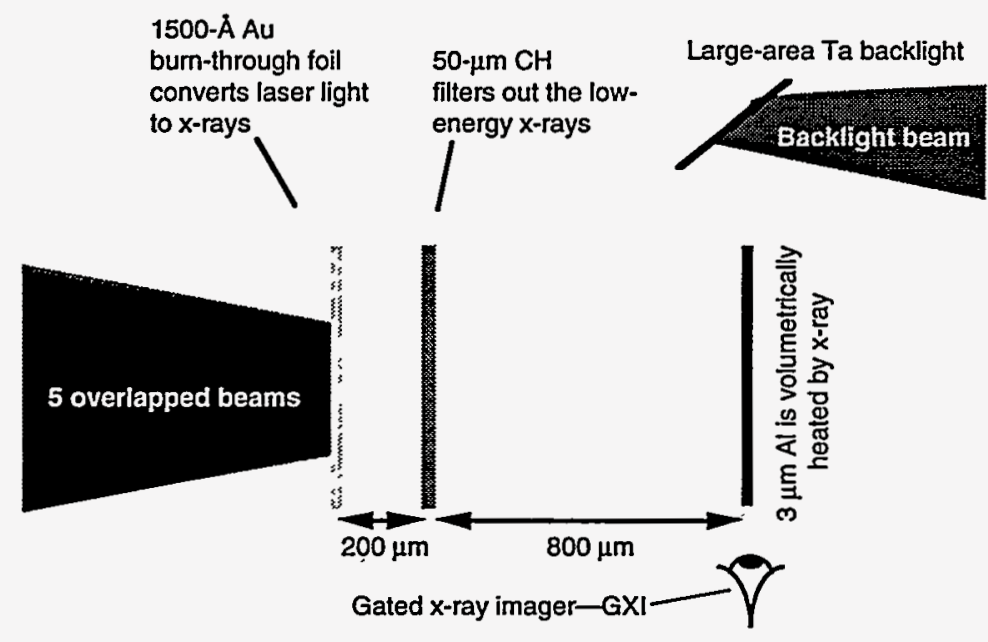

Figure V-8. Schematic of a test bed for hydrodynamic simulations. The aluminum sample is uniformly heated by the x-rays created from a 5-beam illuminated gold burn-through foil. The plastic filter ensures that there is no radiation below the critical density for solid matter. The measurements are made using a multiple-framed $x$-ray imaging camera (a gated $x$-ray imager) looking at the transmission of the large-area backlight passing the target. 
backlighter source. A filter between the backlighter and the aluminum foil minimized heating of the sample foil by the backlighter. Imaging was by a $10-\mu \mathrm{m}$ pinhole array and a gated microchannel plate $x$-ray detector with a total spatial resolution of $15 \mu \mathrm{m}$. Four images can be taken on each experiment, with a temporal resolution of better than $100 \mathrm{ps}$. The images are typically taken at 1-ns intervals.

An example of the reduced data for one such image is shown in Fig. V-9. At approximately $5 \mathrm{~ns}$ the aluminum foil and the plastic foil begin to collide, which is the limiting time for this configuration. Comparison of these experiments with calculations showed reasonable agreement, as was to be expected for such a simple geometry. One such comparison is shown in Fig. V-9. The theoretical prediction of the movement and expansion of the foil would be critical tests of the EOS and opacity of the hydrodynamic simulation. The agreement here is reasonable and would suggest more complicated experimental configurations could be tried. With these initial conditions verified, it will be possible to proceed to more complicated geometries, such as stepped targets, to provide further tests of the hydrodynamics.

\section{Spherical Geometry}

For the spherical geometry case, implosion imaging of ICF capsules, or microspheres, will be presented. A typical implosion sequence is viewed with a $5.3 \times$ magnification spanning $\Delta \mathrm{t}=1.4$ to $1.95 \mathrm{~ns}$. The instrument used to make the measurement is a 12-frame gated pinhole camera denoted WAX. The active element of this multi-frame $x$-ray pinhole camera consists of a microchannel plate (MCP) overcoated with a serpentine (S-shaped) gold microstrip ${ }^{2}$ (see Fig. V-10).

The MCP gain is switched on and off by a 170-ps FWHM 800-V pulse that travels along the serpentine strip. Because a voltage is required to accelerate the electron produced to the rear of the microchannel plate, this imager provides a simple means of obtaining temporal resolution by the choice of voltage pulse shapes.

The WAX has been characterized in situ with short $x$-ray bursts produced by irradiating gold disk targets with tightly focused, 100-J, 20-ps,

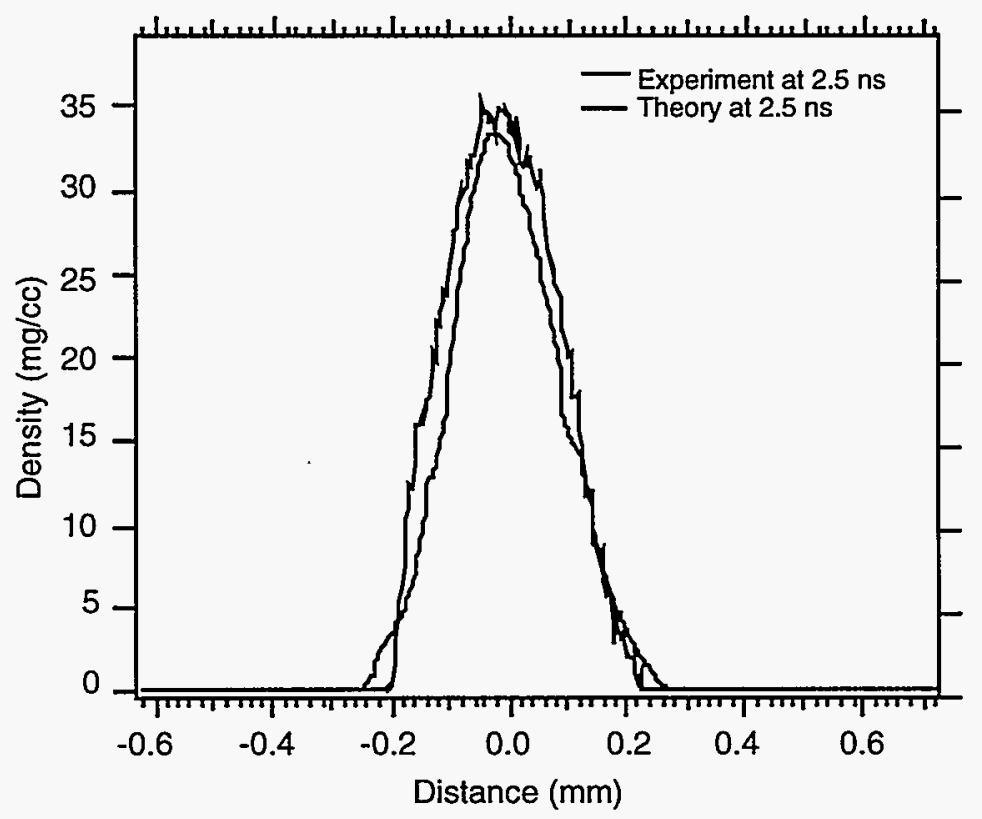

Figure V-9. An example of the data obtained from the experimental test bed for hydrodynamic simulations. The agreement here between experiment and theory is reasonable and would suggest that more complicated experimental configurations could be tried. 


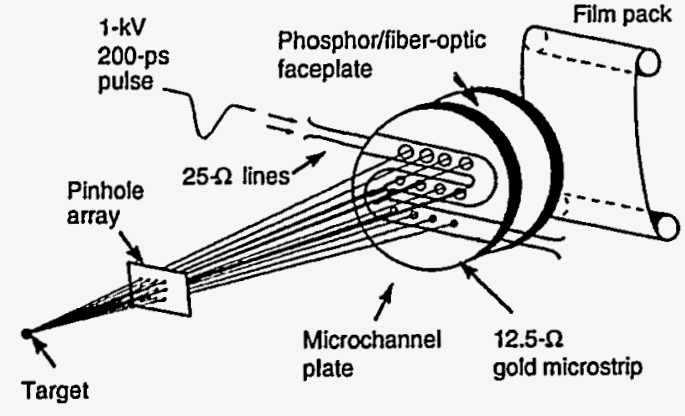

Figure $V-10$. The $W A X$, a 12-frame $x$-ray gated imager. The instrument is capable of taking sequential images on a serpentine (S-shaped) gold strip coated onto a microchannel plate to make it sensitive to $x$-rays. Because a voltage is required to accelerate the electron produced to the rear of the microchannel plate, this imager provides a simple means of obtaining temporal resolution by the choice of voltage pulse shapes.
2- $\omega_{0}$ Nova laser pulses. The measured temporal resolution is $80 \pm 5$ ps FWHM, the voltage pulse propagation speed is $0.55 \mathrm{c}$, and the transverse sensitivity variations are $\leq 10 \%$.

The target consisted of a $470-\mu \mathrm{m}$ diameter polystyrene shell overcoated with a $55-\mu \mathrm{m}$ plastic ablator and filled with $50 \mathrm{~atm}$ deuterium, $0.1 \mathrm{~atm}$ argon, and $0.02 \mathrm{~atm}$ xenon. This fill, as is indicated below, is used to provide a spectroscopic indicator of the composition of the implosion core. A 1-ns square, 17-kJ laser drive in a scale-1 gold hohlraum was used. The interframe spacing is $45 \mathrm{ps}$, or $70 \mathrm{ps}$ between frames separated by a bend. The camera is filtered to pass emission only above $3.5 \mathrm{keV}$, at the argon free-bound and xenon bound-bound photon emission energies.

Figure V-11 shows a typical implosion sequence for a 1-ns square drive pulse, using the WAX diagnostic. The image is filtered to show emission above $3.5 \mathrm{keV}$. In the figure, the capsule, which converges to $50 \mu \mathrm{m}$ diameter, already

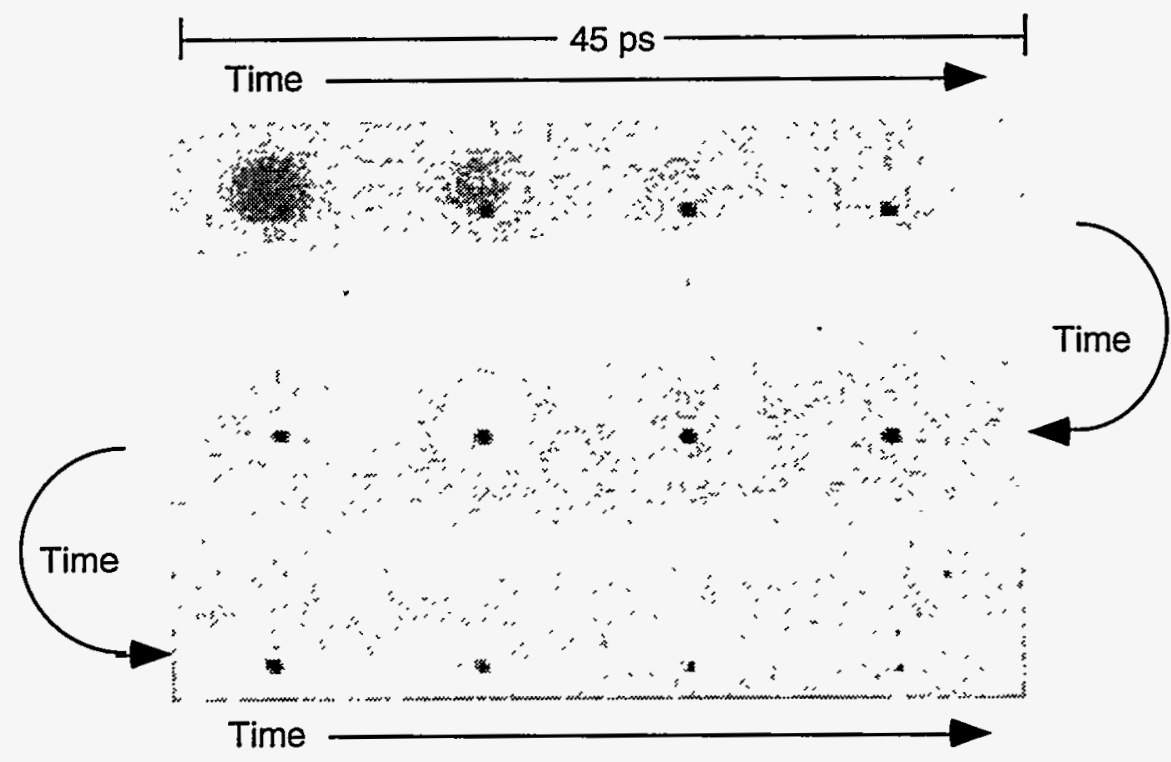

Figure V-11. Typical implosion sequence for a 1-ns square drive pulse, using the WAX diagnostic. The experiment had a $17-\mathrm{kJ}$ drive in scale-1 gold hohlraum-driven $470-\mu \mathrm{m}$ diameter polystyrene shell overcoated with a 55- $\mu \mathrm{m}$ plastic ablator and filled with $50 \mathrm{~atm}$ deuterium, $0.1 \mathrm{~atm}$ argon, and $0.02 \mathrm{~atm}$ xenon. The image is filtered to show emission above $3.5 \mathrm{keV}$. The capsule, which converges to $50 \mu \mathrm{m}$ diameter, already appears in emission in frame 1 (at $\Delta \mathfrak{t}=1.4 \mathrm{~ns}$ ), peaks in emission in the 5 th frame $(\Delta t=1.6 \mathrm{~ns})$, and is disassembling by frame $11(\Delta t=1.9 \mathrm{~ns})$. Residual diffuse gold M-band emission from the cooling hohlraum plasma is also observed through the $50 \%$ laser entrance hole, decreasing in intensity with delay, as would be expected after the end of the laser pulse. 
appears in emission in frame $1(\Delta \mathrm{t}=1.4 \mathrm{~ns})$, peaks in emission in the 5 th frame ( $\Delta \mathrm{t}=1.6 \mathrm{~ns})$, and is disassembling by frame $11(\Delta t=1.9 \mathrm{~ns})$. Residual diffuse gold $M$-band emission from the cooling hohlraum plasma is also observed through the $50 \%$ laser entrance hole, decreasing in intensity with delay, as would be expected after the end of the laser pulse.

Quantitative results for a similar shot with a higher drive of $29 \mathrm{~kJ}$ are presented in Fig. V-12. This experiment used a 1-ns square pulse, 29-kJ drive in scale- 1 hohlraum. Time $=0 \mathrm{ps}$, which is defined as peak emission time, occurs $0.3 \mathrm{~ns}$ after the laser pulse is switched off.

In Fig. V-12, A) shows the evolution of the FWHM of the emitting regions in two orthogonal planes. The $10 \% \mathrm{~m}=2$ asymmetry observed may be due to low- $Z$ patches near the hohlraum equator creating a few percent lower flux at the target equator. B) is a plot of the evolution of the intensity of the imploding core emission and hohlraum plasma emission. After deconvolving the instrument time response, the FWHM of the $x$-ray emission from the implosion is measured at $150 \mathrm{ps}$, in agreement with streaked spectral measurements that have higher temporal resolutions.

\section{Unstable Hydrodynamics}

\section{Planar Geometry}

There are numerous possible areas of investigation for the study of planar instabilities in high-energy-density matter. The two that are discussed here should only be considered a start on two aspects of the problem, and not the defining experiments. In the first case we discuss the growth of an initially imposed perturbation under the influence of a continuous acceleration (i.e., the Rayleigh-Taylor case), while in the second case we treat an interface that has random perturbations and is shocked (i.e., the Richtmeyer-Meshkov case).

\section{Imposed Perturbation-Rayleigh-Taylor}

The first experiment shown is one intended to study large-growth Rayleigh-Taylor instability using a radiation source as the driver. ${ }^{3}$ See
A) X-ray emission

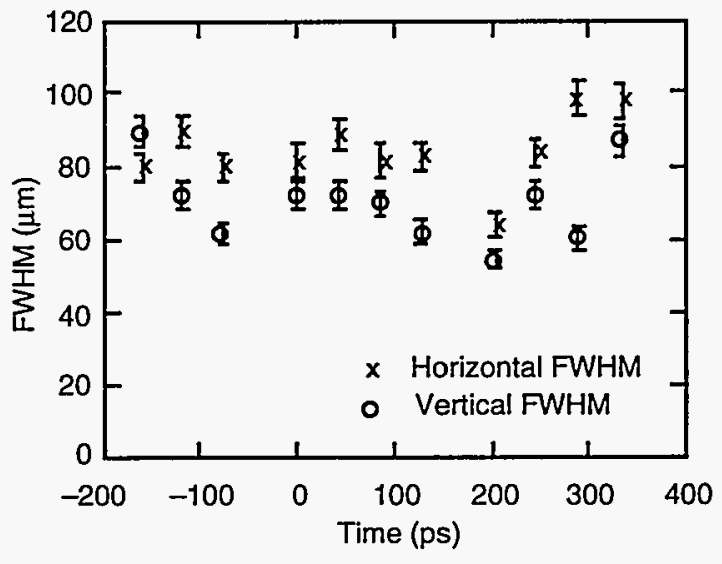

B) Background emission intensity

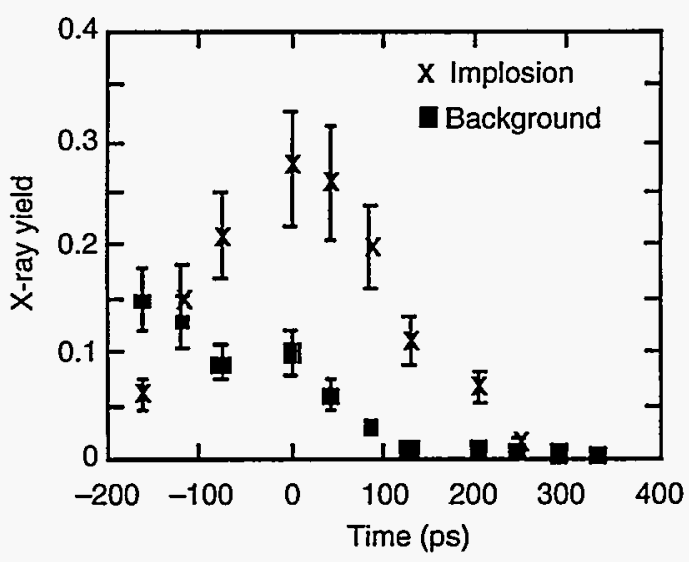

Figure V-12. Time history of an implosion. A) Evolution of spatial FWHM of x-ray emission in two orthogonal planes. B) Plot of the evolution of target and hohlraum back-ground emission intensity. This experiment uses a 1-ns square pulse, $29-\mathrm{kJ}$ drive in scale-1 hohlraum.

Fig. V-13 for a schematic of the experiment. In this type of experiment, planar foils of fluorosilicone are accelerated by $x$-ray ablation. The foil trajectory is measured using edge-on radiography to check the bulk movement of the ablating sample.

In separate experiments, which employed face-on radiography, contrast in the optical depth is measured as a function of time. From this contrast, the evolution of the $50-\mu \mathrm{m}$ wavelength initially impressed sinusoidal perturbations can be deduced. Measurements of the growth of the 


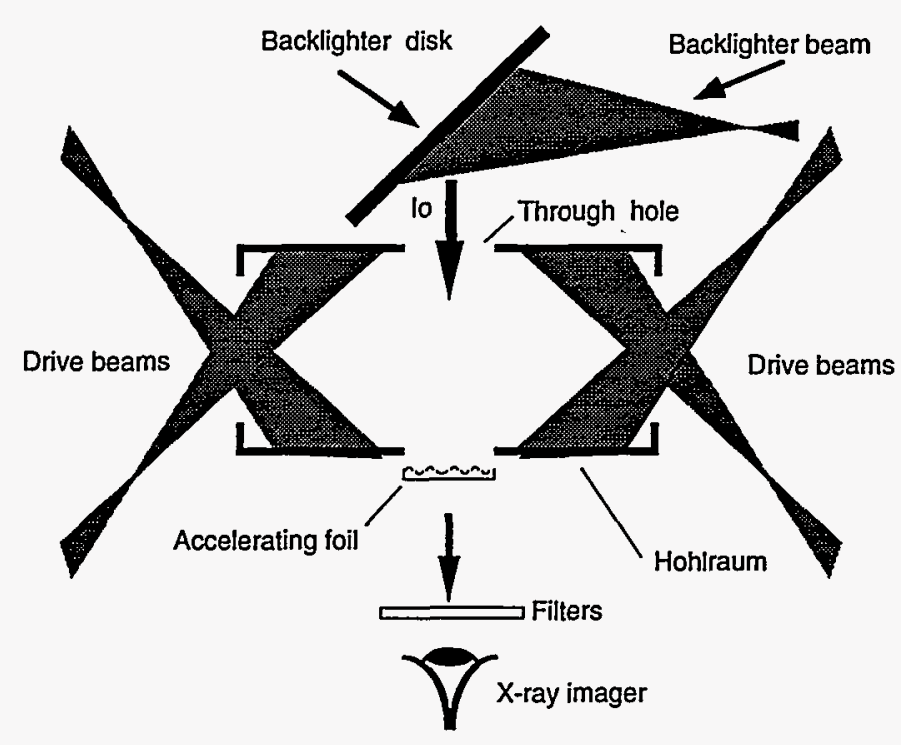

Figure V-13. Schematic of a setup to study large-growth Rayleigh-Taylor instability. The schematic shows the face-on radiography of a target that has been impressed with a sinusoidal pattern. The target is mounted on the wall of a cylindrical gold hohlraum with the surface perturbation facing inwards. The laser beams enter the hohlraum via holes in the end, generating an $x$-ray drive. As the foil accelerates by $x$-ray ablation towards the $x$-ray imager, another laser strikes the backlighter disk. This generates a back illumination of $x$-rays, which pass through the hohlraum and the accelerated foil. Modulations in the foil areal density translate to modulations in exposure at the imager.

perturbation were made using both an $x$-ray streak camera, which shows the continuous onedimensional growth of the perturbation during the experiments, and a two-dimensional image with a frame time of $100 \mathrm{ps}$. The two-dimensional image shows the full foil used, in order to verify the planarity of the foil.

Figure V-14 A) shows the streak camera image, with time shown in the vertical and position across the foil shown in the horizontal. The image shows the optical depth modulation of the accelerated foil and indicates that the foil becomes, overall, increasingly bright due to thinning. The thinning is due in turn to the formation of the "bubble-and-spike" shape characteristic of the nonlinear Rayleigh-Taylor regime. Figure V-14 B) shows a two-dimensional image of the foil, taken at $2.6 \mathrm{~ns}$, for a frame duration of $100 \mathrm{ps}$. This data is essential to ensure that there is no transverse distortion of the foil.

The quantitative data is obtained by taking intensity traces transverse to the grooved structure at different times. In Fig. V-15 we show a sample of such a series of traces. Note that the curves, which represent different times in the evolution, are offset vertically for ease of comparison. At early times the contrast is small and still sinusoidal, indicating that the RayleighTaylor instability is in the linear regime. Late in time, the contrast is larger and distinctly nonsinusoidal, exhibiting the bubble-and-spike shape characteristic of the nonlinear Rayleigh-Taylor regime. The rapid flattening of the modulations in the top two curves results from the burnthrough when the bubbles break out of the back side of the foil. At this point, the spikes are still being ablated away, but no longer can be replenished by matter flowing down from the bubbles.

These experiments have been extended from the single mode example shown here to multiple mode experiments, ${ }^{4}$ and to buried interfaces with imposed mode structures. ${ }^{5}$ The limiting case of a random set of perturbations at a buried interface requires a somewhat different technique. 
A) One-dimensional image

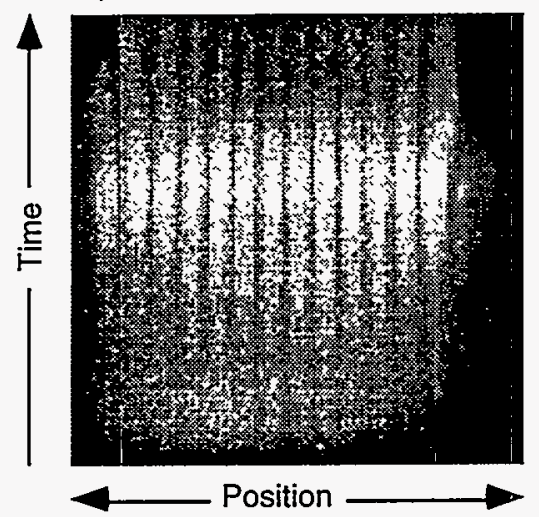

B) Face-on two-dimensional image

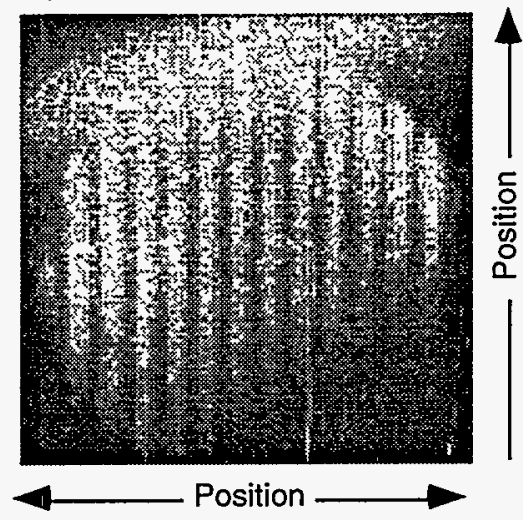

Figure V-14. Face-on streak-camera image of the backlight source absorbed by the foil with initial perturbation. A) A one-dimensional streak camera image with time increasing in the vertical direction and position across the foil in the horizontal direction. The end of the bright spot occurs when the backlight ceases to radiate effectively. B) The face-on two-dimensional image of the foil indicating little, if any, distortion of the foil. This image was taken with a 100-ps frame time and occurs at $2.6 \mathrm{~ns}$ after the start of the drive.

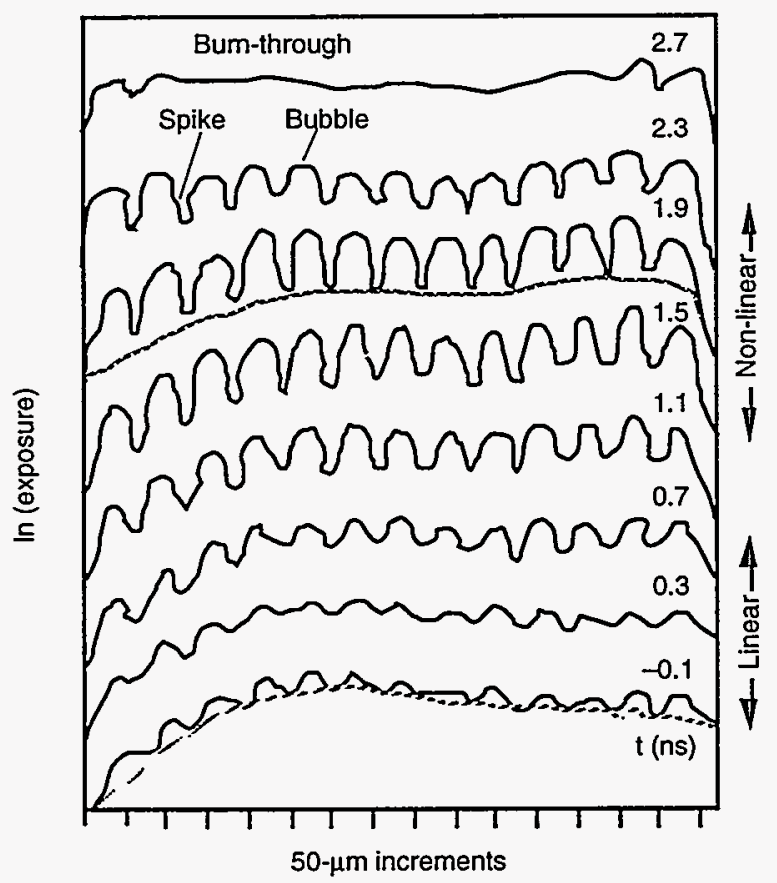

Figure V-15. Intensity traces for the accelerated foil with an initial perturbation of $50 \mu \mathrm{m}$ wavelength and $1.9 \mu \mathrm{m}$ amplitude sinusoidally imposed on the surface. The curves are offset in the vertical direction to allow simple comparison. The backlight intensity variation across the foil and as a function of time is noted by the dashed line at the initial time and later in the pulse.
Imbedded Random Surface-Rayleigh-Taylor

The second case we discuss under planar unstable flow concerns an imbedded interface with random perturbation and an impulsive shock acceleration. Here the technique of point projection spectroscopy is used. The experiment is illustrated in Fig. V-16. A planar target with an ablator section and a foil section, shown in Fig. V-16, is radiatively accelerated normal to its surface. The drive was produced by focusing $6-10 \mathrm{~kJ}$ of $0.35-\mu \mathrm{m}$ light in $1 \mathrm{~ns}$ from eight of the beams of Nova into a hohlraum. A planar shock of $\sim 60 \mathrm{Mbar}$ pressure with a rise time of less than 50 ps propagated through the layered target, accelerating it to about half of its final velocity and driving an instability between the ablator and foil materials.

See Fig. V-17 for a schematic of the geometry of the point projection spectroscopy using a backlight. After a suitable delay, the target was backlit by a flash of $x$-rays produced by focusing another beam, with $200 \mathrm{~J}$ of $0.35-\mu \mathrm{m}$ light in a pulse length of 100 to $200 \mathrm{ps}$, onto a $22-\mathrm{mm}$ coated fiber. The fibers were coated with $1-\mu \mathrm{m}$ thick co-sputtered mixtures of gold, bismuth, and platinum, chosen to give bands of $x$-ray emission in the 2- to 3-keV region. Because the flash came from a small source, it will project a shadow of the target. However, it was first Bragg-reflected 


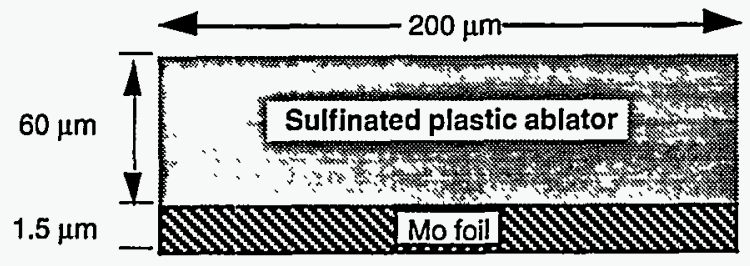

Figure V-16. Diagram of target for study of imbedded random surface using point projection spectroscopy. The target consists of an ablator section of low-density sulfinated plastc and a high-density molybdenum section. The dimensions of the low-density sulfinated plastic/high-density molybdenum target are shown.

from a potassium pthallate crystal before it was used to project a shadow of the target in motion onto x-ray film. Since the crystal reflects only when Bragg's law is satisfied, the shadow has spectral as well as spatial resolution in one direction, the $x$ direction in Fig. V-17. Spatial resolution is also obtained in the $\mathrm{z}$ direction, normal to the disk, so that the $\mathrm{z}$ distance moved by various materials in the package could be measured.

The materials in the target are chosen to have photoabsorption edges in the wavelength range of the shadow of the package-e.g., the sulfur $K$ and the molybdenum $\mathrm{L}_{\mathrm{II}}$ and $\mathrm{L}_{\mathrm{II}}$ shown in Fig. V-18. The measured change in absorption across the edge and the known increase in opacity allow the areal density of the material to be evaluated. Mix of the target materials is detectable by the overlap, if any, of the photoabsorption edges. This is schematically illustrated in Fig. V-18, where the no-mix target shows minimal density mismatch at the interface, while the mixed case shows a density mismatch.

A serendipitous feature of this technique is that high-opacity absorption lines form close to the cold edges as material is heated and ionized, acting as a sensitive, albeit qualitative, indicator of the position of the materials.

The accelerated target consisted of a 200- $\mu \mathrm{m}$ diameter, $\sim 1.5-\mu \mathrm{m}$ thick disk of molybdenum accelerated by a $60-\mu \mathrm{m}$ thick, lower density ablator of sulfinated plastic. Manufacturing resulted in a finish on the molybdenum/plastic

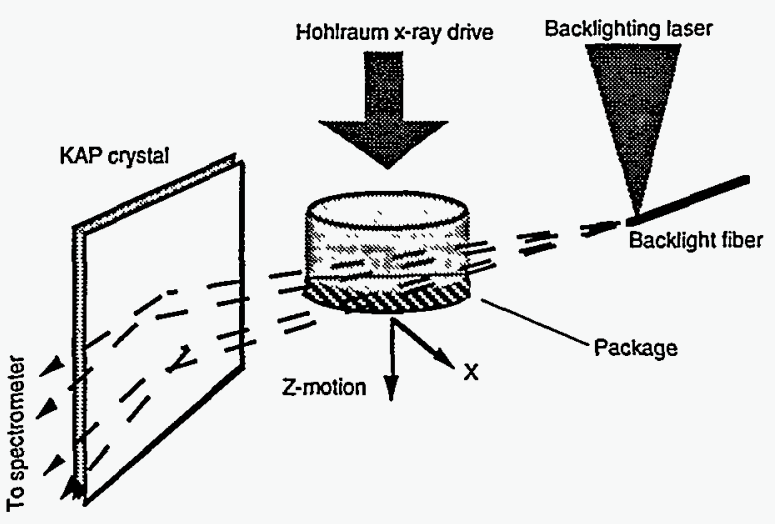

Figure V-17. Schematic of point projection spectroscopy technique, adapted for study of the mixing of interfaces. The layered target is accelerated by the $x$-ray drive from a hohlraum, driving an instability between the ablator and foil materials. After a suitable delay, a point backlight is created, and the backlight $x$-rays pass through the accelerated sample. The backlight $x$-rays impinge on a crystal, which provides spectral dispersion. The dispersed $x$-rays are then imaged on $x$-ray film.

interface of typically $0.1 \mu \mathrm{m} \mathrm{rms}$, with random wavelengths of $1-5 \mu \mathrm{m}$. The high initial-density mismatch in this experiment, measured by the Atwood number, was $~ 0.8$ at the ablator-foil interface, and was derived from the density mismatch between the molybdenum $(10.4 \mathrm{~g} / \mathrm{cc})$ and the ablator plastic $(1.36 \mathrm{~g} / \mathrm{cc})$.

Most of the detailed information about the target is obtained from the absorption spectra of the materials in the target. Figure V-19 is an example of an image in which the target was backlit $7 \mathrm{~ns}$ from the start of the 1-ns drive. The image shows absorption as a function of wavelength and position in the horizontal ( $x$ ) direction, and as a function of position only in the vertical ( $z$ ) direction. The target image captured in flight can clearly be seen to have moved upward, away from the edge of its support. A spatial fiducial made of $25-\mu \mathrm{m}$ wire provides a reference for the position of the package when the radiograph is taken. It also demonstrates the $20-\mu \mathrm{m}$ source size which, when combined with the $10-\mu \mathrm{m}$ motional blurring, gives a limiting spatial resolution of $\sim 30 \mu \mathrm{m}$. 


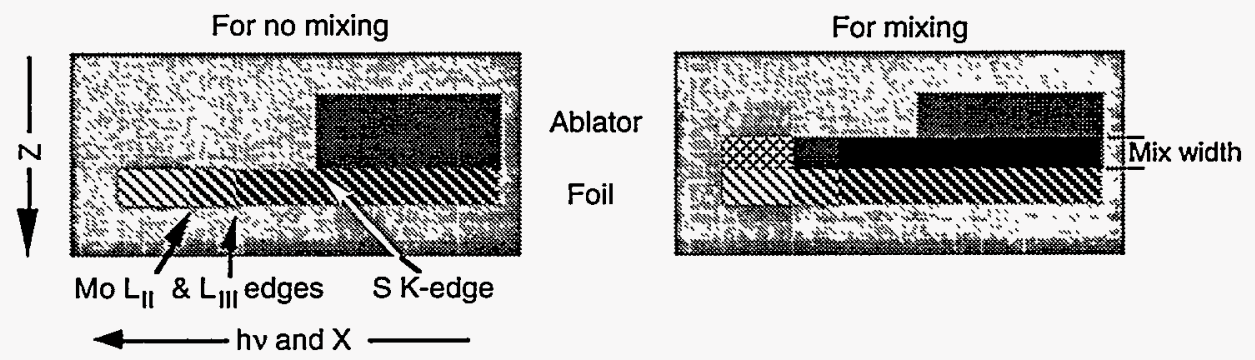

Figure V-18. Spectrometer images from point projection spectroscopy of imbedded random surface. On the left are the spectral signals for an unmixed (or no-mix) target, where little mix should occur, and on the right are signals for a mixed target. The film contains the $x-z$ image of the target, with the added advantage that the spectrum provides an indicator of where, in the $z$ direction, the elements of interest are found. The materials in the target are chosen to have photoabsorption edges in the wavelength range of the shadow of the package (i.e., sulfur $\mathrm{K}$ and the molybdenum $\mathrm{L}_{\mathrm{III}}$ and $\mathrm{L}_{\mathrm{II}}$ shown). Mix of the target materials is detectable by the overlap, if any, of the photoabsorption edges. Note that the no-mix target shows minimal density mismatch at the interface, while the mixed case shows a density mismatch.

The traces at $\mathrm{z}=413 \mu \mathrm{m}$ in Fig. V-19 show a clear sulfur $\mathrm{K}$ edge and a weaker molybdenum $\mathrm{L}_{\mathrm{III}}$ edge. The middle trace at $\mathrm{z}=463 \mu \mathrm{m}$ shows both sulfur $K$ and molybdenum $L_{\text {III }}$ edges, whereas the upper trace at $z=496 \mu \mathrm{m}$ shows a large molybdenum $L_{\text {III jump }}$ and a small sulfur $K$ jump. These three traces demonstrate that the foil and the ablator are intermixed over a substantial spatial region.

The axial distribution of areal density of the materials of the package comes from the $K$ and $\mathrm{L}_{\mathrm{II}}$ edge-jump measurements shown in Fig. V.-19. If $T_{b}(a)$ is the spectral transmission below (above) the edge then the areal density of the material along the viewing direction is given by

$$
\int \rho(l) d l=\ln \left(T_{b} / T_{a}\right) / \Delta(\mu / \rho)
$$

where $\Delta(\mu / \rho)$ is the change in the mass absorption coefficient across a photo-absorption edge (2220-217 $\mathrm{cm}^{2} / \mathrm{g}$ and $1990-545 \mathrm{~cm}^{2} / \mathrm{g}$ for the sulfur $\mathrm{K}$ and molybdenum $\mathrm{L}_{\mathrm{III}}$ edges, respectively) and $l$ is the viewing length.

From Fig. V-19, the diameter of the package at the observation time is $\sim 360 \mu \mathrm{m}$, which is larger than the initial 200- $\mu \mathrm{m}$ diameter because of decompression. Because the sulfur $\mathrm{K}$ and molybdenum $\mathrm{L}_{\mathrm{III}}$ edges are close to the center of the package in Fig. V-19, this gives an upper limit on $l$ of $360 \mu \mathrm{m}$. The measurements that depend on the sulfur $\mathrm{K}$ and molybdenum $\mathrm{L}_{\mathrm{III}}$ edges of the axial distributions of areal density of sulfur and molybdenum are shown in Fig. V-20 A). The limitation on the lowest density measurable is $\sim 0.03 \mathrm{mg} / \mathrm{cm}^{2}$ for the penetration of the sulfinated plastic into the molybdenum. This limit arises from errors in the background, as the overall transmission is high ( 0.7). For the molybdenum penetration into the sulfinated plastic, the lower limit on detectability is $0.2 \mathrm{mg} / \mathrm{cm}^{2}$, which arises from the low overall transmission, $\sim 0.05$. Clearly an overlap region of $\sim 150 \mu \mathrm{m}$ exists that is much larger than the system resolution.

One potential problem in interpretation would be the loss of planarity, due to either bowing or tilting of the target. However, this apparent mixing of the ablator and the highdensity foil is not due to bowing, as can be inferred from Fig. V-19. Nor is it due to tilting, as another view at $44^{\circ}$ is used to check target orientation. Other effects, such as the ablator blowing by the molybdenum foil, could be producing the apparent mix, so to minimize this possibility control experiments were performed in which little mix should occur.

The target for these no-mix shots had the same ablator as the high-mix shots, of density 1.36, but the molybdenum foil was replaced by a $15-\mu \mathrm{m}$ chlorinated plastic foil, 


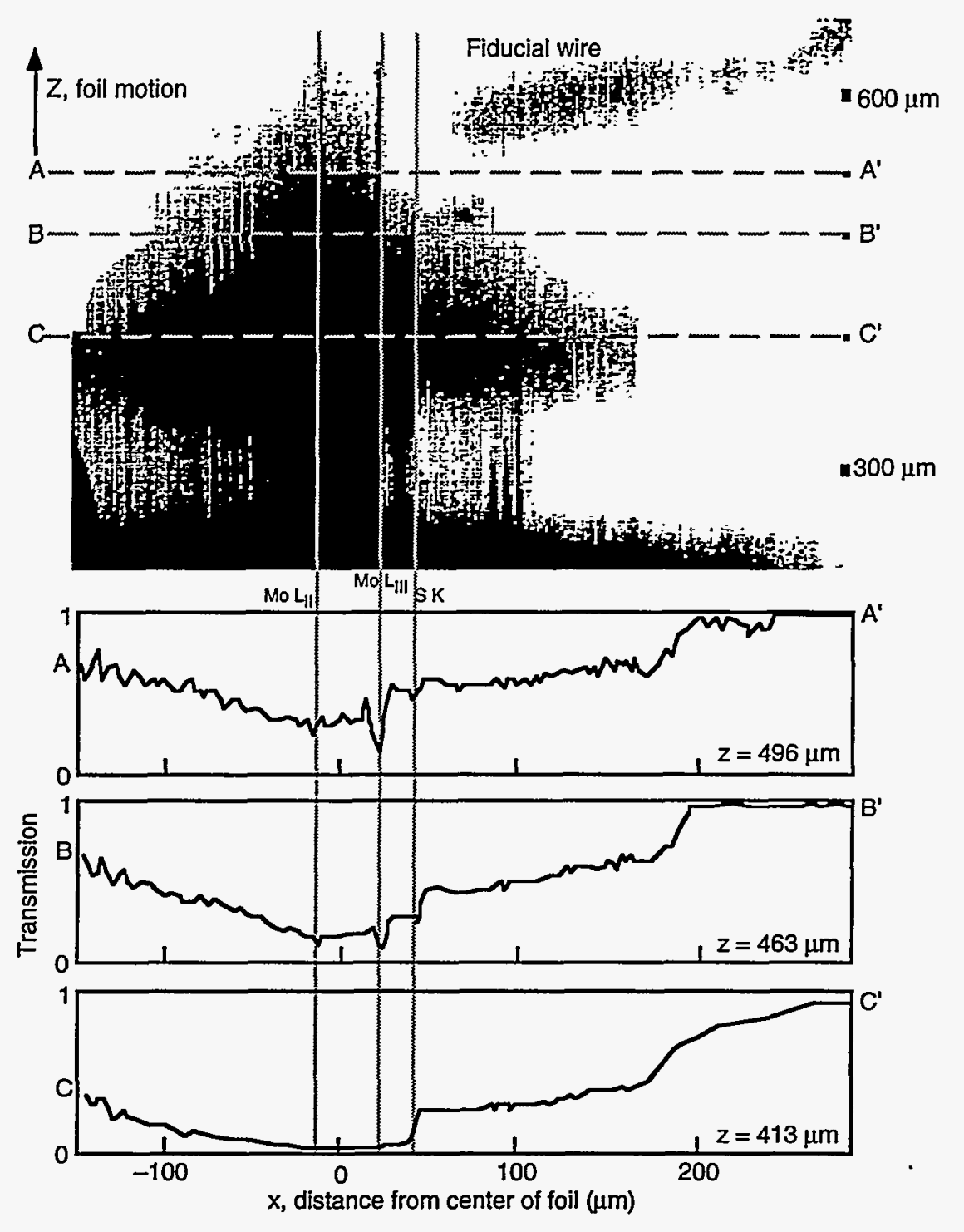

Figure V-19. Radiograph of a sulfinated plastic ablator driving a molybdenum foil, above, with three intensity traces. Radiograph was taken at $7 \mathrm{~ns}$ with a backlight pulse length of $150 \mathrm{ps}$. The traces were taken at three positions. Zero on the $z$ (vertical) axis is the rear surface of the package at $t=0$, and on the $x$ axis zero is the original center of the foil. The image clearly shows the sulfur K edge, the molybdenum $\mathrm{L}_{\mathrm{III}}$ lines, and the edge of the package.

made from polychlorostyrene of density 1.23 . The initial density mismatch is very small, having an Atwood number of -0.05 , so little mixing is expected. The mixing of the foil and ablator in Fig. V-20 (B) is less than $40 \mu \mathrm{m}$, which is approximately the experimental resolution.

\section{Imbedded Interface-Richtmyer-Meshkov}

To show another possible configuration that is suitable for instability studies, we describe an experiment that addresses techniques to study the Richtmyer-Meshkov instability. The experimental configuration is the same as depicted in Fig. V-1, but the density interface is planar, without the hemispherical protrusion. The inter-face has a roughness machined into a high-density brominated plastic, and it is this roughness that seeds the Richtmyer-Meshkov instability.

The experiments to develop the techniques are in progress, but preliminary data is shown in 
Fig. V-21. This image was taken about $8 \mathrm{~ns}$ after the initiation of the drive. The shock is moving from top to bottom, and the reference grids used to provide spatial fiducials in the experiments are visible on either side of the image. Note that this image does not take into account the spatial profile of the backlighter, which is roughly Gaussian, and the effect of the cylindrical package geometry on the transmission.

In Fig. V-22 we show a $100-\mu \mathrm{m}$-wide vertical trace taken on the centerline of the image in Fig. V-21 (the jagged curve) together with a

A) High-mix case

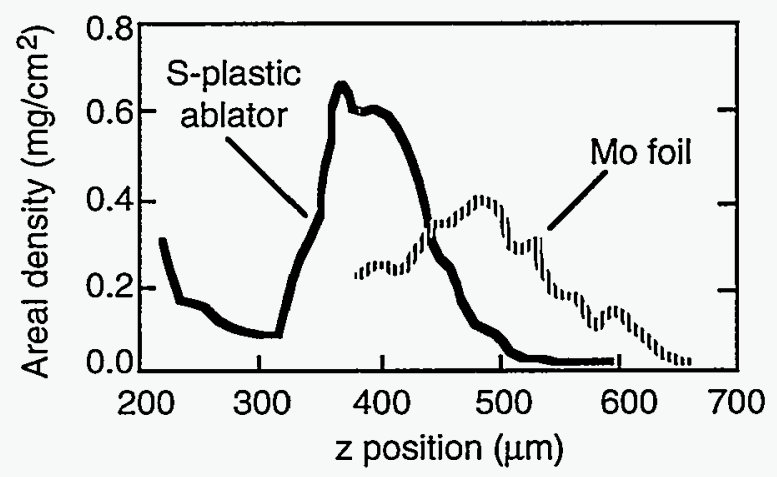

B) No-mix case

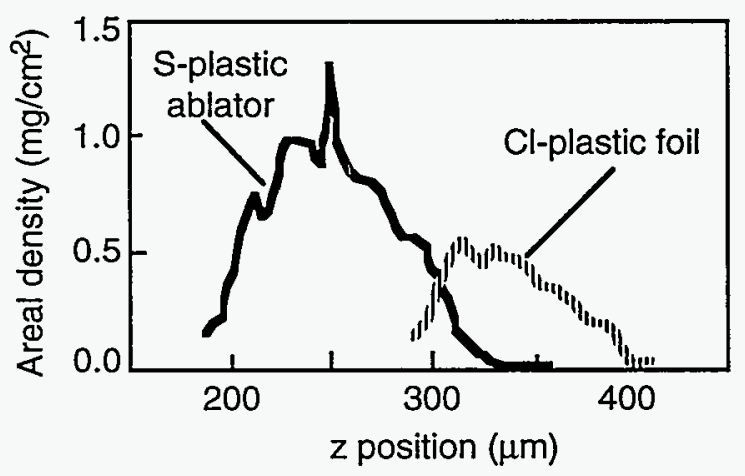

Figure V-20. Axial distribution of mass areal densities for A) the high-mix case of Fig. V-19 and $B$ ) the no-mix shot, both measured at the package centers. The mass areal densities of the sulfur in the plastic, the molybdenum, and the chlorine in the plastic are plotted. The fact that there are traces of the two different spectroscopic markers in the same z-position indicates that mixing has occurred.
Gaussian fit to the backlighter spatial profile (the smooth curve). The fit to the backlight spatial dependence was found using the unshocked region in the image.

The trace was normalized by the backlight spatial dependence to remove most of the backlighter non-uniformity, and the results are displayed in Fig. V-23. In Fig. V-23 the level region from $\sim 570$ to $825 \mu \mathrm{m}$ demonstrates that the Gaussian profile is a reasonable approximation of the spatial shape of the backlight. The two arrows denote the locations of the calculated interface and shock locations.

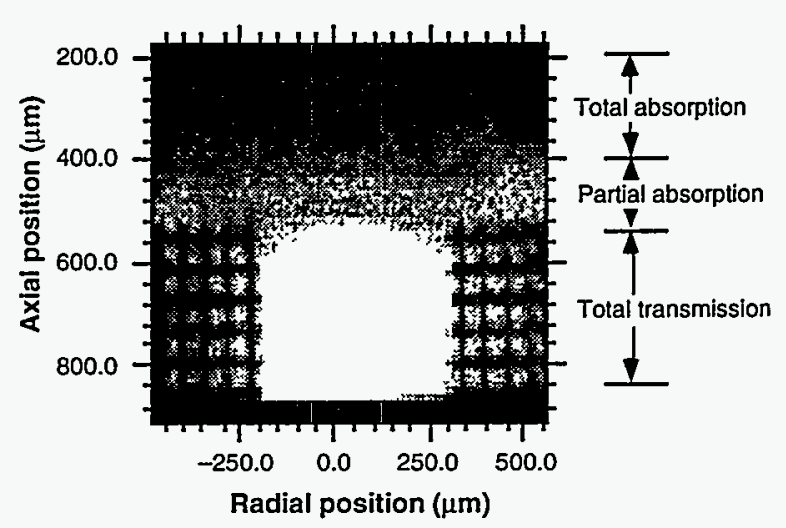

Figure V-21. Gated $x$-ray image of planar Richtmyer-Meshkov mix target at about $8 \mathrm{~ns}$.

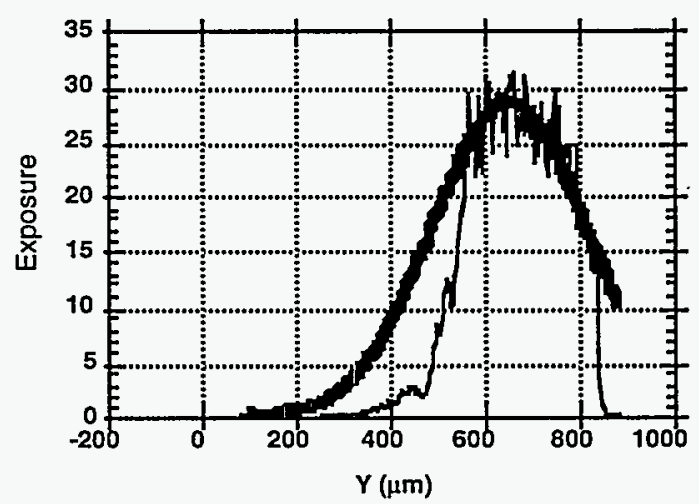

Figure V-22. A $100-\mu \mathrm{m}$-wide vertical trace of the centerline of the image in Fig. V-21 (thin jagged line), and the accompanying fit to the backlight (thick line). 
It is apparent from Fig. V-23 that a simulation can reproduce the gross features of the experiment. Sharp drops in the exposure delineate the edge of the higher-opacity regions in the shock-compressed foam and then in the brominated-doped plastic. The width of the features is the distance over which these transitions occur. For a perfectly flat shock, the measured shock width should be negligible. We measure a shock width of $\approx 15-20 \mu \mathrm{m}$, which is of marginal interest because the diagnostic resolution is $12-15 \mu \mathrm{m}$.

The NIF would provide larger scales and relieve the severe constraints on spatial dimension that are faced with the present-day high-energy lasers. On the other hand, the calculations are in substantial disagreement for the width of the mixed region for this interface. The two-dimensional simulations indicate a mix width of less than $15 \mu \mathrm{m}$, while the measurement indicates a width of 25-30 $\mu \mathrm{m}$. Again, the NIF will provide a test bed for experiments of this type. In the interim, experimental techniques will continue to be developed to obtain a deeper understanding of the physical processes involved in the experiments and how these limit our ability to measure the quantities of interest.

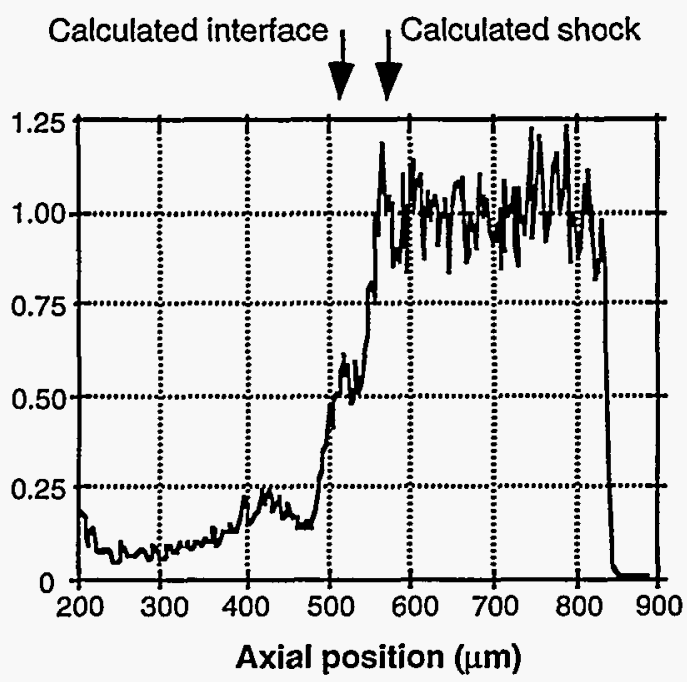

Figure V-23. Vertical trace of the image in Fig. $V-21$ normalized by the backlight.

\section{Spherical Geometry}

The most obvious method to study spherical unstable flow is to attempt to make the usually stable implosion unstable in a controllable way. To do this, the use of perturbed microspheres was perfected. The microsphere, or capsule, shown in Fig. V-24 was a "bumpy" ball equipped with micron-size perturbations imposed on the outer plastic ablator surface, with a chlorinedoped pusher (i.e., the material at the interface with the gas core) and argon-doped 50-atm deuterium gas.

Fig. V-25 shows a single $12.5 \times$ magnification, $12-\mu \mathrm{m}$ resolution image of an implosion, using the bumpy ball, taken 0.2 ns after peak emission. The image was taken by the WAX instrument discussed in Section III, Experimental Capabilities, Subsection A. Note that the figure shows the positions of the beams on the hohlraum wall relative to the image. The experiment used 1-ns square, $17-\mathrm{kJ}$ laser drive

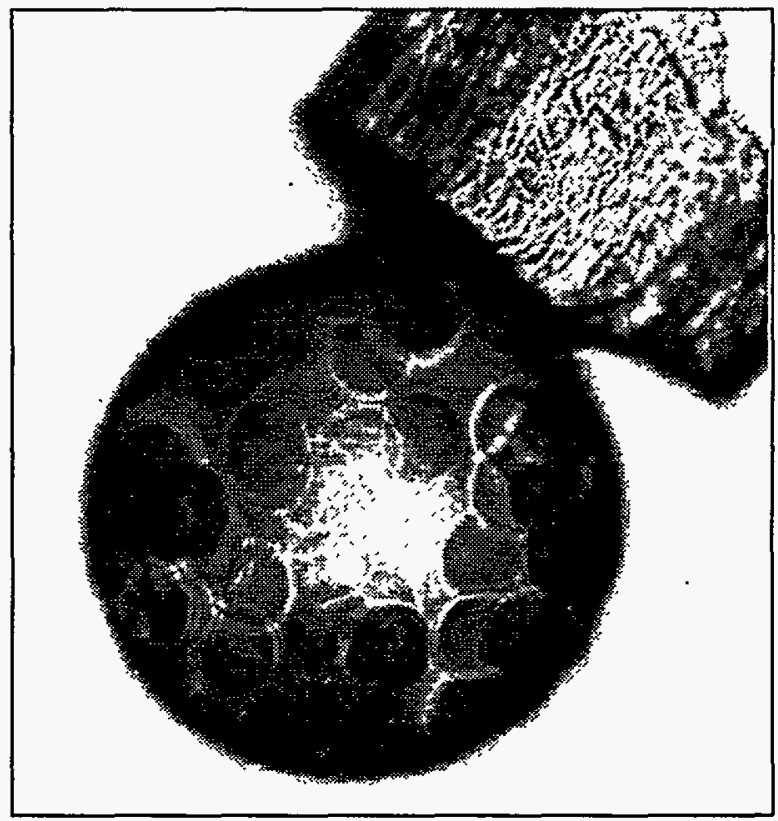

Figure V-24. Machined "bumpy" ball used in implosions. The bumps, which are random, are placed on the surface of the microsphere by laser irradiation. (The cylindrical object to the . upper right side is there to hold the microsphere for processing.) 
in a $2550-\mu \mathrm{m}$-long gold hohlraum. A concave pentagonal shape, the $m=5$ mode, is clearly observed. The nodes, which correspond to regions of lower flux, occur between laser spots. The $m=5$ symmetry is observed on almost all 1-ns square implosions.

In addition, Fig. V-25 shows evidence of higher emission from the edges of the capsule than from the center, commonly defined as limb brightening. The measured polar-averaged radial trace in Fig. V-26 shows the analyzed data that suggests that the evolution of the pusher-gas interface can serve as a measure of the degree of mix of the gas and the pusher, and might be diagnosed by time-resolved limb imaging.

As a further monitor of the mixing of the interface between the solid-density pusher and the gas fill in an implosion, doping of the pusher with a high-Z material can both provide information on the pusher conditions and act as a diagnostic for mixing of the pusher into the gas region. ${ }^{6}$ Using the same type of bumpy ball as shown in Fig. V-24, observations have been made of the emission from various dopants included

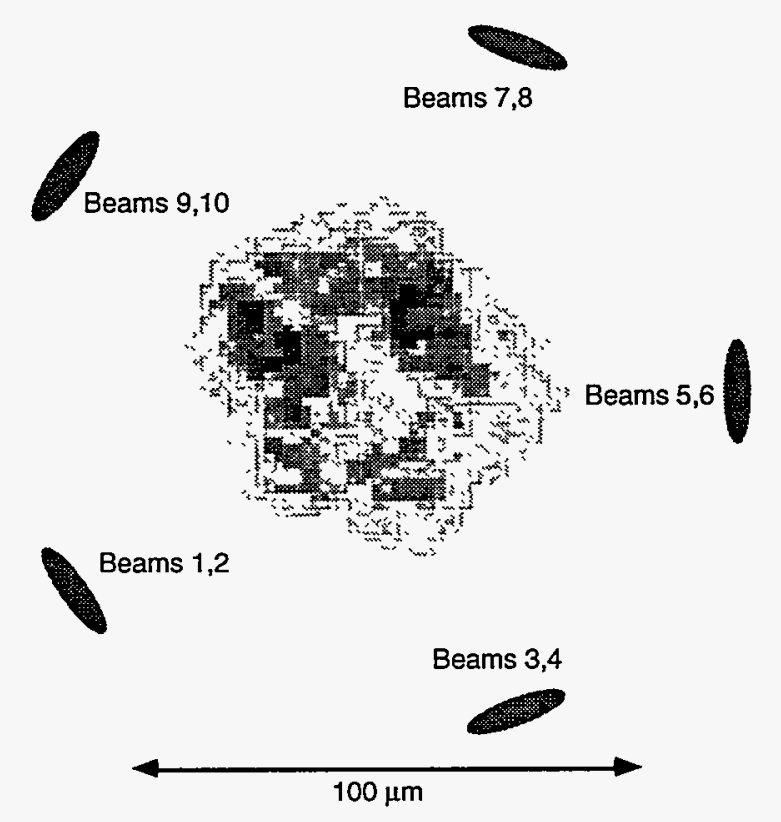

Figure V-25. Expanded view of an implosion image taken using the WAX. The image shows a concave pentagonal $(m=5)$ asymmetry, as well as limb brightening (higher emission from the edges of the capsule than from the center). into the innermost region of the pusher. Fig. V-27 shows $x$-ray streak camera data from both bumpy and smooth microspheres. The dopant in the pusher is $1 \%$ chlorine by number. The microsphere with the bumpy surface has significantly brighter chlorine emission and weaker argon emission than the microsphere with the smooth surface. This is an indication that some of the pusher material has been mixed into the hot gas.

Further extensions of the technique for inferring mix into gas cores permit higher- $Z$ $K$-shell emitters to be used, as these spectra are simpler to interpret. Advances in target fabrication have expanded the choices of possible pusher dopant to include several metals.

Figure V-28 shows the results of another set of experiments with and without bumpy surface finishes, using iron as the dopant in the pusher. In the figure the microsphere with imposed bumps is shown to have a substantial helium-like iron $1 s^{2}-1 s 2 p$ emission feature, while the smoothsurfaced microsphere shows no evidence of the iron emission.

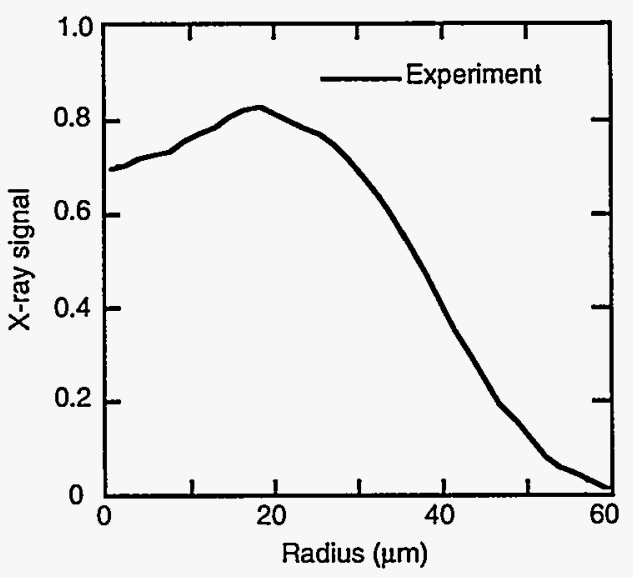

Figure V-26. Measured trace quantifying the limb brightening seen in Fig. V-25. The comparison is only qualitative because absolute timing is not known and the target surface roughness is not fully characterized. 


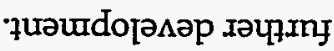

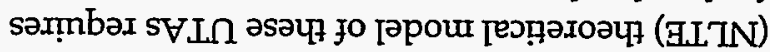
um!̣

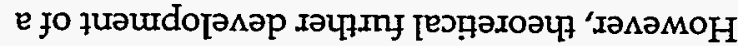

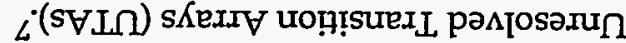

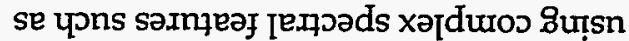

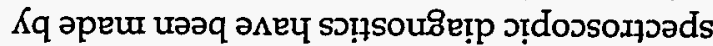

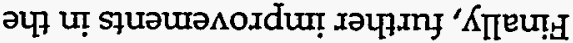

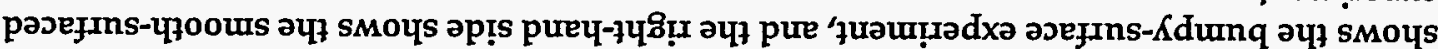

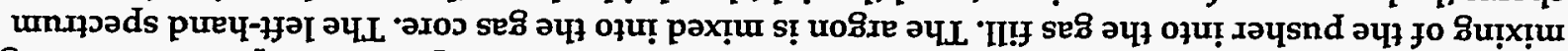

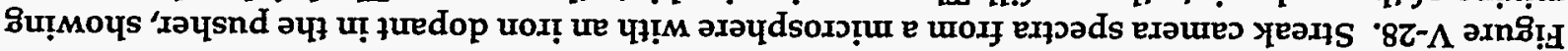

yloous

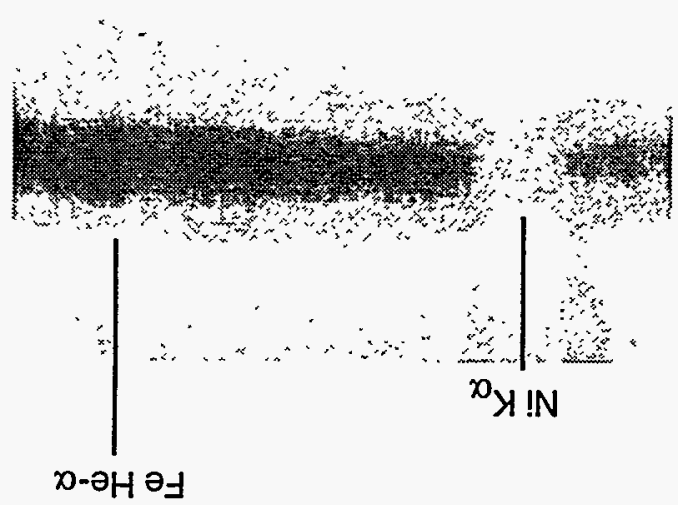

Kdung

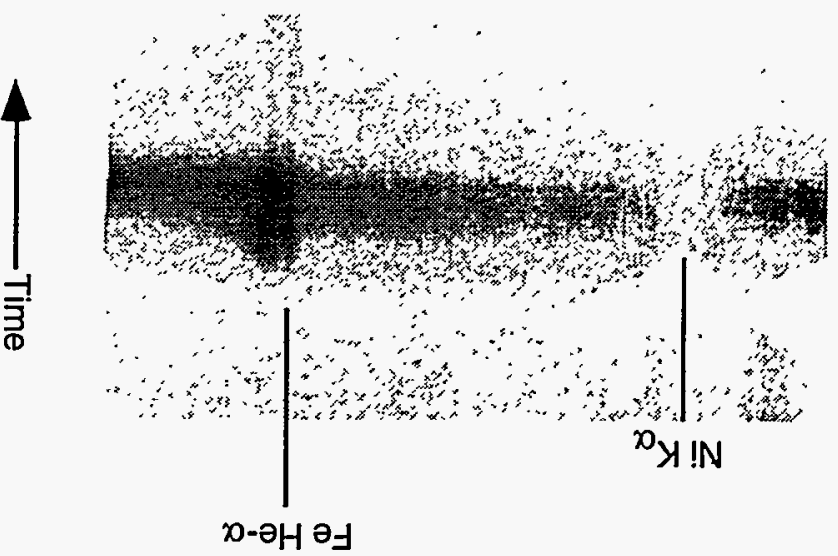

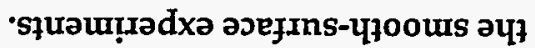

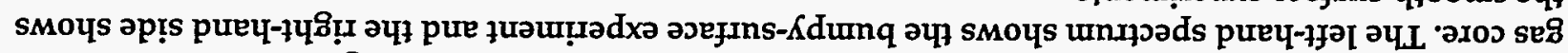

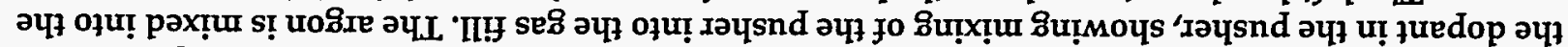

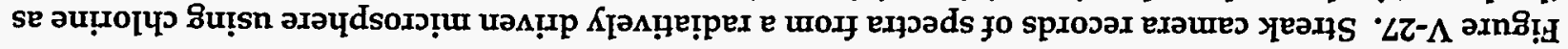
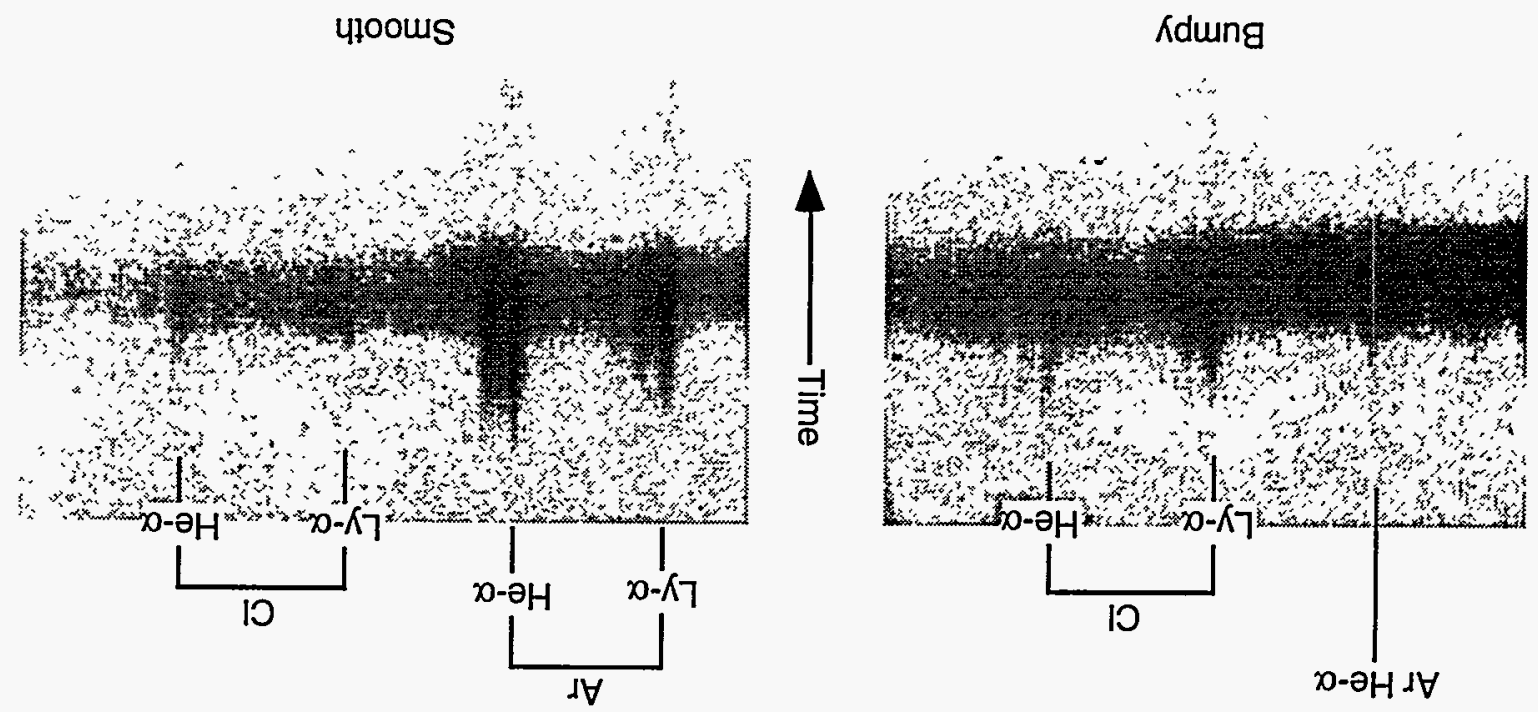


\section{Future NIF Experiments}

In the following, we discuss a number of topics that are among the most promising for experimental investigation under conditions that can only be created at the NIF. We have organized these topics according to the type of flow to be considered and the material conditions present. Thus, the issues to be considered that involve stable flow are hydrodynamic flow at high Mach numbers, shock-shock and converging shock propagation, shock motion through inhomogeneous media, and shockboundary interactions in the strong shock regime, including the relationship to cratering.

Unstable flow problems that are uniquely addressable with the NIF involve, for example, the study of the onset and development of instabilities such as Rayleigh-Taylor, RichtmyerMeshkov, or Kelvin-Helmholtz. The occurrence of these instabilities in blast waves, and in supernova explosions, where they play an important role in mixing the products from different regions, can also be studied in laboratory experiments. Other experiments that could possibly be considered concern the study of radiation condensation and secondary instabilities. Finally, the onset of turbulence and the influence of vortex dynamics in a medium of finite compressibility is an emerging field with important possibilities.

\section{Stable Flow}

This first section on stable flow will discuss the study of strong-shock hydrodynamics in the presence of material boundaries, inhomogeneities, and another strong shock. These studies, including shock-shock interactions, Mach stems, and possibly nonequilibrium gas dynamics analogies and similar phenomena, are all classical problems in fluid dynamics.

The possibility of performing precise experiments in this field is of great interest because of the prospect of benchmarking numerical models; understanding certain space physics, chemistry, and cratering phenomena; and checking exact solutions that are valid in the strong-shock limit. The shock interactions would represent an extension of work done in shock tubes and similar facilities, but potentially at significantly higher Mach numbers.

The analogies with non-equilibrium flows would depend upon the burn process of an ignition target, and are not possible on present lasers. The shocks for stable flow studies are created by the interaction of a strong radiation field with a material. The interaction is created by direct drive, indirect hohlraum heating, or acceleration and subsequent impact of a flyer foil. ${ }^{8}$ The transition from radiation energy deposition to hydrodynamic shock motion is therefore also a topic of considerable interest. Note that there are instabilities that can occur at the material interfaces for some of the topics discussed here, and these are related topics of investigation that will be discussed separately in the section on unstable flow.

Finally, the general area of magnetohydrodynamics holds the potential of some interesting phenomena that could be investigated using the NIF's capability to deposit large amounts of energy into small volumes or areas in very short times. Possible topics for future consideration include generation of electromagnetic interference, enhancement of magnetic fields through compression, and a "photo-voltaic battery."

\section{Shock-Shock Interactions}

Shocks created by a two-beam laser incident onto aluminum targets that propagate toward each other through a nitrogen atmosphere have been recently studied. ${ }^{9}$ Figure V-29 shows a schematic from an experiment to investigate the interaction of two blast waves (see Fig. V-30 for the data). In the experiment, two lasers are . focused onto two 2.5-mm diameter aluminum rods in a nitrogen atmosphere. The ends of the irradiated rods blow outward into the nitrogen, creating two shocks that interact in the medium. Density and temperature in the shock-shock interaction region were determined by spectral line broadening and intensity ratio methods. In addition, an attempt was made to determine the physical processes occurring as the shocks collide and then either pass through each other or reflect. 


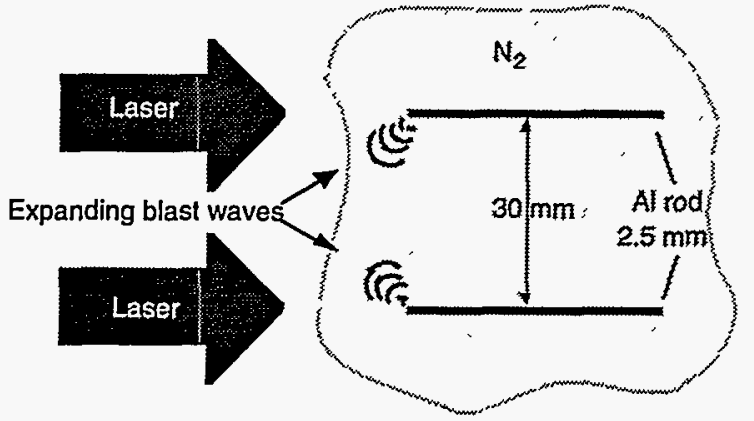

Figure V-29. Schematic of a shock-shock interaction experiment. Two lasers are focused onto two $2.5-\mathrm{mm}$ diameter aluminum rods in a nitrogen atmosphere. The ends of the irradiated rods blow outward into the nitrogen, creating two shocks that interact in the medium. Spectroscopy of NII and NIII transitions provides in-situ measurements of the electron density through line broadening. The time dependence of the electron density in the shock-shock interaction region is determined by observing the midplane with a streak camera coupled to a visible spectrometer.

Figure V-30 is a graph of electron density vs time in $\mu$ s for two cases, a single wave (data points denoted with $x$ ) and a colliding wave (denoted with circles). The curve for the singlewave case illustrates the rapid onset of the shocked density enhancement and subsequent rapid decrease. The curve for the colliding-wave case illustrates the density enhancement of the shock-shock interaction and the slow decay of the density after the shock.

On the NIF it should be possible to extend these quantitative techniques to obtaining data at extreme shock conditions. Further, the NIF will make it possible to access the increased shock strength needed for different nonlinear regimes.

The interaction of strong shocks is a typical nonlinear flow problem in shock hydrodynamics and for extreme shock conditions, numerical solutions have been the only source of information in the past. Increasing the shock strength will permit different nonlinear regimes to be accessed. A similar study, equally important for hydrodynamic theory, is that of a converging strong shock caused by focused acoustic energies such as those formed in pellet

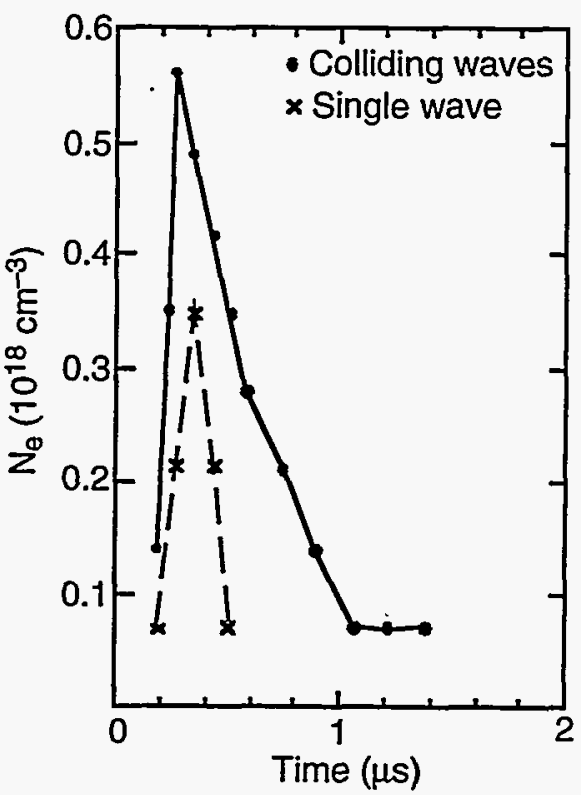

Figure V-30. Graph of electron density vs time in $\mu$ s for two cases, a single wave and a colliding wave. The curve with the data for a single shock wave (data points denoted with $x$ ) shows the rapid onset of the shocked density enhancement and subsequent rapid decrease. The curve showing data from the colliding wave (data points marked with circles) illustrates the density enhancement of the shock-shock interaction and the slow decay of the density after the shock.

implosion or bubble collapse. ${ }^{10}$ This should also be possible with a large-spatial-scale laserenergy incident.

\section{Shock-Boundary Interactions}

There are a series of possible experiments on the interaction of a shock wave in a medium that has high acoustic impedance and is bounded by a medium of low acoustic impedance. Examples are underwater or underground blast waves that impact a planar surface with a gas, or a planar shock passing over a bubble. If the shock propagates in the strong-shock regime, the rarefaction generated either by the shock at the point of contact with a planar surface or by a plane shock at the point of contact with a spherical bubble can, in certain geometries, overtake and distort the impinging shock. This is analogous to the transition from a regular 
reflection to a Mach reflection for flow over a wedge, except here the reflected wave is a rarefaction instead of a shock. This effect has been predicted, ${ }^{11}$ but the experimental evidence has all been produced in the gas phase with lessthan-strong shocks. ${ }^{12}$

Self-similar solutions show that the rarefaction generated at the interface by an expanding spherical shock can also overtake the shock both along the surface and straight down into the medium, so the effect is interesting both for spherical shocks generated by point sources and for plane shocks. An important application of these studies is in the understanding of rangeto-effect of the shock waves created by underground or underwater plate tectonics. Another application is found in the possibility of experimentally measuring shock propagation in inhomogeneous media, which is relevant to the astrophysical problem of the hydrodynamic interaction of shock waves with interstellar clouds. ${ }^{13}$

Figure V-31 shows the results of simulations of an interstellar cloud being shocked, illustrating propagation of a shock in an inhomogenous medium. The first frame shows the initial planar shock moving at velocity $v_{b}$ toward a cloud of radius $\mathrm{a}_{0}$. The cloud is 10 times as dense as the interstellar medium, and the shock in the cloud has a Mach number of 10 . As the shock transits it "crushes" the cloud, and in re-expansion a complicated combination of instabilities occursRichtmyer-Meshkov due to the impulsive acceleration of the initially perturbed cloudintercloud boundary as well as Kelvin-Helmholtz and Rayleigh-Taylor instabilities.

At a time of $8 a_{0} / v_{b}$ (the second frame) the cloud has clearly suffered large-scale unstable flow. Importantly, by time $12 \mathrm{a}_{0} / \mathrm{v}_{\mathrm{b}}$ (third frame) the cloud is distorted and axially flattened. At this later time there is a plume of fragments that contains $70 \%$ of the cloud mass. This process of distortion and axial flattening continues until the cloud is largely dispersed, with axial dimensions reaching five time the original size and a transverse dimension reaching two times the original size. This kind of experiment, in compressible inhomogenous media where
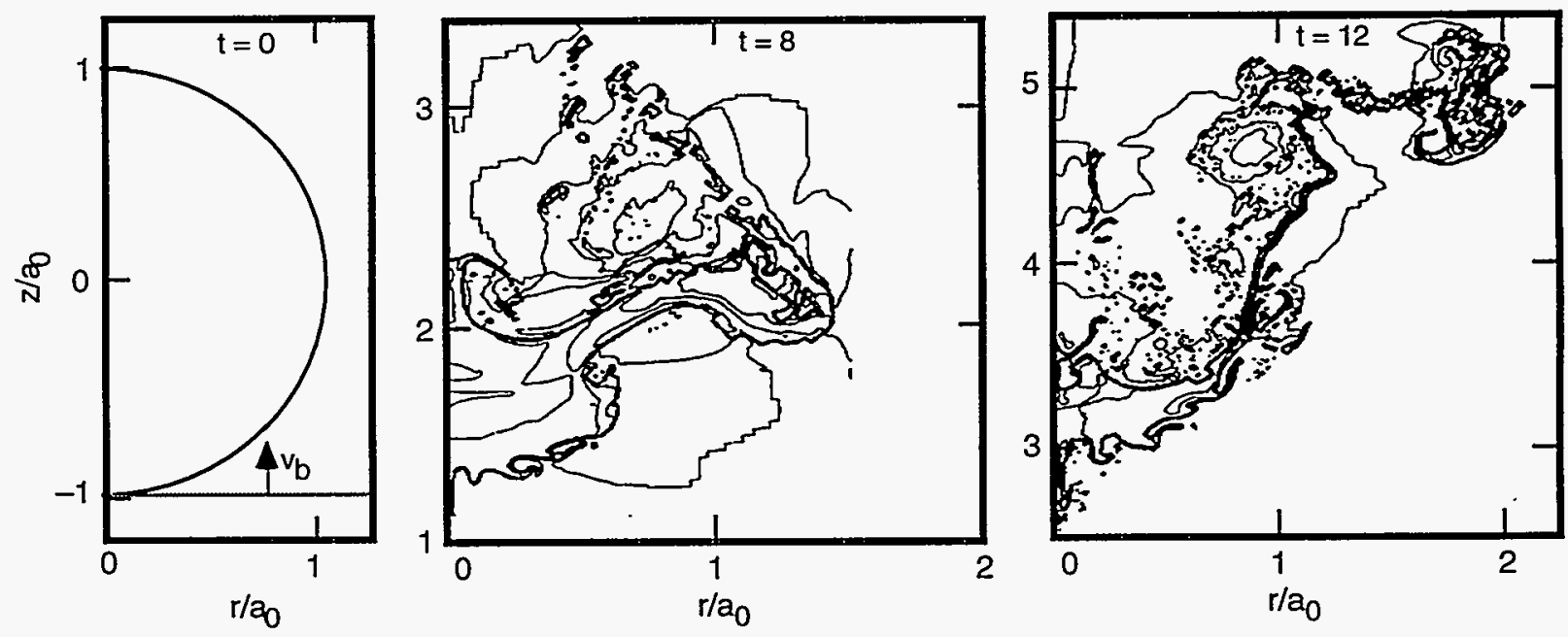

Figure V-31. Propagation of a shock in an inhomogeneous medium is illustrated by these simulations of an interstellar shock propagating through a cloud. The first frame shows the initial planar shock moving at velocity $v_{b}$ toward a cloud of radius $a_{0}$. As the shock transits the cloud it "crushes" it, and in re-expansion a complicated combination of instabilities occurs. At a time of $8 a_{0} / v_{b}$ (the second frame) the cloud has clearly suffered large-scale unstable flow and by time $12 a_{0} / v_{b}$ (third frame) the cloud is distorted and axially flattened. This process of distortion and axial flattening continues until the cloud is largely dispersed, with axial dimensions reaching five time the original size and transverse dimension reaching two times the original size. 
radiation plays a role and time scales for development of the full complement of instabilities are possible, requires the scale of the NIF, where energy is available to provide not only high-Mach-number shocks but diagnostic capability.

\section{Hypersonic Flow}

Hypersonic flow problems with Mach numbers above 20 can be studied with laserproduced plasmas. This is a regime unattainable with present-day wind tunnels or shock tubes. Three-dimensional flow at these speeds is associated mainly with the reentry of space vehicles or the flight of a projected ramjet airplane. Theoretical calculation of this flow involves difficult questions of flow past particular surface shapes (noting that cones ${ }^{14}$ are of particular interest, of course). The chemistry of the wakes created by a body moving through a fluid with hypersonic speed is important for the understanding of the effects of flight or reentry through the atmosphere, particularly with respect to the creation of plasmas or the interaction with constituents such as the ozone layer.

\section{Impact Cratering}

Many phenomena of interest in impact cratering occur on temporal and spatial scales that are large compared to those of the impacting object. As a result, the impact can be modeled as a point source of high energy and momentum density and the mass of the object can be neglected. ${ }^{15}$ This situation can be modeled by the deposition of focused laser energy in small spheres of high- $Z$ material.

Alternatively, flyer foils that can generate prodigious shocks and post-shock pressures-on the order of gigabars-could be used. Thus, although the formation of a crater by the impact of a meteor on a surface seems to be entirely different from the formation of a crater by a surface blast, there are possibilities for using this method to model impact craters.

A study of concentrated impacts on surfaces ${ }^{16}$ shows that scaling laws apply to the shape of both craters formed by impact and those formed by surface energy deposition. Therefore, the cratering created by an intense radiation source or flyer foil would be useful in studying the scaling of the crater shape and inferring the shape of impact craters.

A series of proof-of-principle experiments using focused laser energy to deposit energy in a grout mixture (which simulates the soil sample) below a planar interface with the atmosphere has been performed, ${ }^{17}$ with results that suggest that further research in this area with higher energy densities would be of great interest. Further, it should be pointed out that the effect of gravity was also simulated, as well as geological layering effects in separate experiments. The experimental schematic of these experiments is shown in Fig. V-32. The experiment was set up to explore the hydrodynamic response of a simulated soil.

The experiment demonstrates several facts:

- The energy of the laser is captured in a small volume inside the grout block used to simulate soil.

- The mass and energy involved in the deposition did not escape through the laser entrance hole.

- The energy density provided by $4 \mathrm{~kJ}$ of laser energy in $1 \mathrm{~ns}$ is sufficient to vaporize the gold target and the surrounding grout.

The ability of the laser to deposit large amounts of energy in a small volume without creating residual gases (which would be a byproduct of the same experiment performed with high explosive) indicates its utility as a simulation source. On a NIF-sized laser, the

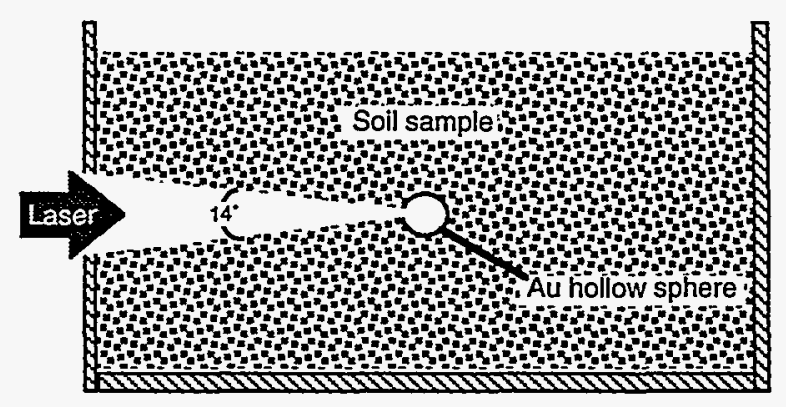

Figure V-32. Setup to explore the hydrodynamic response of a simulated soil. The experiment demonstrates employs one laser beam focused onto the rear of the gold hollow sphere embedded with soil, or grout, sample. 
amount of energy deposited and the in-situ diagnostic potential make it possible to investigate hydrodynamic response possible in real-time experimental configurations.

Figure V-33 shows images showing the results of the experiment to simulate hydrodynamic response of soil described in Fig. V-32. Image (A) is a photograph of the post experiment box of grout formed of a $16 \times 16 \times 16$ $\mathrm{cm}$ cube, built up of squares of $3 / 16$-inch aluminum plate. The initial cavity had a $1.5-\mathrm{mm}$ radius and was buried $6 \mathrm{~cm}$ deep. It captured approximately $4 \mathrm{~kJ}$ of energy from the laser, enough for the requisite vaporization of the gold sphere. Less than $200 \mathrm{~J}$ of laser radiation was observed to escape the target.

The top surface is crazed, as seen in image A), and slightly bowed up. Flash radiographs were taken, and image B) shows a top view, clearly showing the cavity and the entry cone. Note the profusion of radial cracks and the faint but definite indication of tangential (spherical) cracks. The final cavity was approximately $2 \mathrm{~cm}$, which is consistent with cube-root scaling from strong explosion data. This diagnostic is an

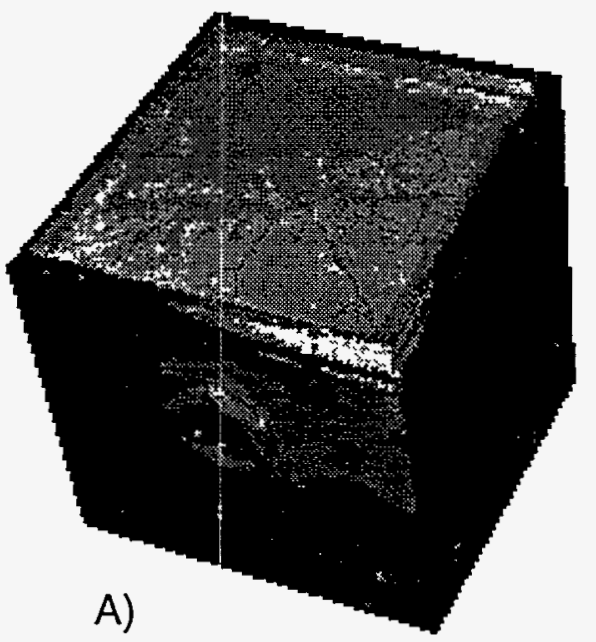

example of the detail that can be learned from scaled experiments that would be very difficult, if not impossible, to get from full-scale experiments.

\section{Scaled Radiative Energy Coupling}

The coupling of explosive energy into hydrodynamic motion is quite different depending on whether the energy originates from a chemical reaction or from the impact of a flyer foil, or is deposited by a radiative energy source. The study of the physical mechanisms involved in the latter case (the coupling of energy deposited by a strong radiative source above, on, or below the surface of dense matter and fluids) is of special interest. This energy coupling varies from zero for sources at large heights above the surface to $100 \%$ for deeply buried sources.

The shape of the coupling curve as a function of source position, of course, has to do with questions of range-to-effect and cratering, but is also of scientific interest for the study of the exact mechanisms determining the coupling. It has been proposed that the coupling of energy proceeds through three stages: first, the stripped-

Fure V-33. Experimental results of hydrodynamic response of soil. Image A) shows the post experiment box formed of a $16 \times 16 \times 16 \mathrm{~cm}$ cube, built up of squares of 3/16" aluminum plate. The initial cavity had a $1.5-\mathrm{mm}$ radius and was buried $6 \mathrm{~cm}$ deep. It captured approximately $4 \mathrm{~kJ}$ of energy from the laser, enough for the requisite vaporization of the gold sphere. Less than $200 \mathrm{~J}$ of laser radiation was observed to escape the target. The top surface is crazed, as seen in image A), and slightly bowed up. Flash radiographs were taken, and image B) shows a top view, clearly showing the cavity and the entry cone. Note the profusion of radial cracks and the faint but definite indication of tangential (spherical) cracks. 
sphere stage of complete ionization, then thermal wave propagation, and finally a momentumconserving transition to shock propagation and cratering.

A low-temperature thermal wave propagation has been observed by monitoring the ionization front produced by the irradiation of a foam material with an $\mathrm{x}$-ray source produced by laser irradiation of a gold foil. ${ }^{18}$ Similar studies have investigated shock propagation ${ }^{19}$ and the possible formation of a Marshak wave. ${ }^{20}$ A study of these mechanisms that play a role in ultimately transforming the incident radiant energy into kinetic energy of ground motion is of great interest.

\section{Unstable Flow}

In this section, we will describe representative fluid dynamics experiments that are designed to study the growth of flow instabilities and are appropriate for study with the NIF laser. Areas of unstable fluid dynamics that are readily, and uniquely, addressable by the NIF include the study of Rayleigh-Taylor, Richtmyer-Meshkov, and Kelvin-Helmholtz instabilities in both planar and non-planar geometries, for the linear, weakly nonlinear, and turbulent regimes.

Some experience has been acquired in previous experiments on this topic, but its larger scales, longer times, and longer drive duration, which are constant themes for hydrodynamic experiments, make the NIF well-suited to extend those investigations. See Subsections A, B, and C for descriptions of several state-of-the-art experiments to illustrate how the NIF will affect hydrodynamics experiments.

These instabilities, and the ensuing mixing that usually follows their development, are of interest because of their occurrence in inertial fusion, astrophysics, energy conversion technology, and the medical applications of lasers, to name a few areas. Another area of unstable flow which has recently become of significant interest is the study of compressible turbulence and mixing in high-speed jets and shear layers. Recent work indicates that turbulence may be qualitatively different at high Mach numbers and/or high compressibilities.
The NIF will provide an excellent facility to generate high-Mach-number flows, typically either jets or shear layers, which may be used in these investigations.

\section{Classical Instabilities}

The instabilities to be discussed in this section are those that refer to the growth of perturbations at a fluid interface. The type of instability in the flow is determined by the origin of the perturbation. If it.is related to the action of shear or constant acceleration, the instability is generally referred to as Kelvin-Helmholtz or Rayleigh-Taylor, respectively, and the growth is exponential in time. An impulsive acceleration caused by a shock normal to the interface causes the Richtmyer-Meshkov instability, which, in the absence of shear or acceleration, then grows linearly in time.

These phenomena have been extensively studied in the past to understand the resulting interface motion and material mix, which are important effects in inertial confinement fusion ${ }^{21}$ and for clumping in supernova explosions. ${ }^{22}$ Nevertheless, important questions remain, because often experiments do not agree with the theory or with the numerical simulations in certain flow domains, especially at high compression. ${ }^{23}$ Experiments on the NIF with long drive times and large spatial scales are expected to be especially important for the understanding of these instabilities.

\section{Other Instabilities}

There are a number of problems associated with other instabilities, and with combined instabilities, that are also appropriate for study with laser-driven flow. The most obvious candidate is the instability associated with the propagation of a strong shock through a uniform gas, which may play an important role in the structure of supernova blast wave propagation and in the formation of stars and galaxies. Theoretical disagreements have generated considerable controversy in the past, but the recent observation of these instabilities ${ }^{24}$ in a laser-driven blast wave indicates that more research is needed on this topic. Figure V-34 shows a schematic of an experiment performed to investigate an unstable Taylor-Sedov blast wave, 
or shock, along with the data generated by the experiment.

The experiment consists of a $200-\mathrm{J}$ laser incident on a 6- $\mu \mathrm{m}$ thick plastic foil. The laser is $5 \mathrm{~ns}$ in duration and is focused to an $880-\mu \mathrm{m}$ diameter spot. The irradiated plastic will ablate and expand at greater than $10^{7} \mathrm{~cm} / \mathrm{s}$ into the surrounding fill gas. The gas fill is chosen to change the adiabatic index, $\gamma$, from 1.3 to $\sim 1.0$ for nitrogen and xenon fills, respectively. $B$ ) shows an optical dark-field shadowgraph of the shock wave at $243 \mathrm{~ns}$ in the nitrogen fill. The image has a frame time of $5 \mathrm{~ns}$ or less, and effectively freezes the shock front. The image shows that the shock front, the dark circle, is uniform and the material behind the shock is homogeneous. In C), the xenon fill shock front shows a completely different front, not uniform, with inhomogeneity in the material behind the shock. The considerable controversy on this subject, the extension to denser systems, and the higher energy densities available on the NIF will make these types of instabilities ripe for the NIF.

The NIF is uniquely suited to producing the strong shocks with large spatial extent that are required for these studies. In addition, combined Rayleigh-Taylor, Kelvin-Helmholtz, and Richtmyer-Meshkov instabilities have been theoretically described in cases where an oblique shock is preceded or followed by an acceleration and/or shear. ${ }^{25}$ Such combined instabilities, which occur whenever horizontal and vertical shear are simultaneously significant, are important for describing flow in winds and ocean currents, and for the fanning of smoke plumes. Experiments that could cleanly examine the dominant features of these phenomena are highly desirable and well-suited to the larger-scale flows the NIF will be able to generate.

There is a wide class of two-dimensional flows that become unstable in the transition to three-dimensional flow. These secondary instabilities occur because two-dimensional finite amplitude waves can be exponentially unstable to three-dimensional perturbations. This is another classical fluid dynamics problem. The study of these instabilities by Poiseuille or plane Couette flow has generally been performed in a regime where material compressibility has no influence. The effect of material compressibility

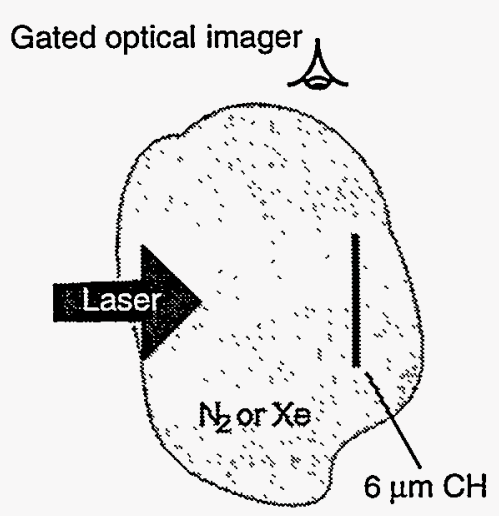

A)

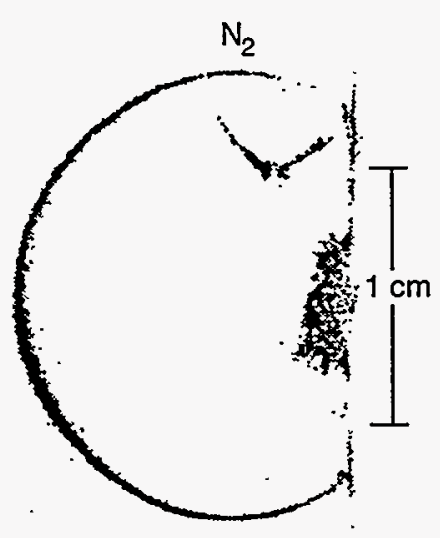

B)

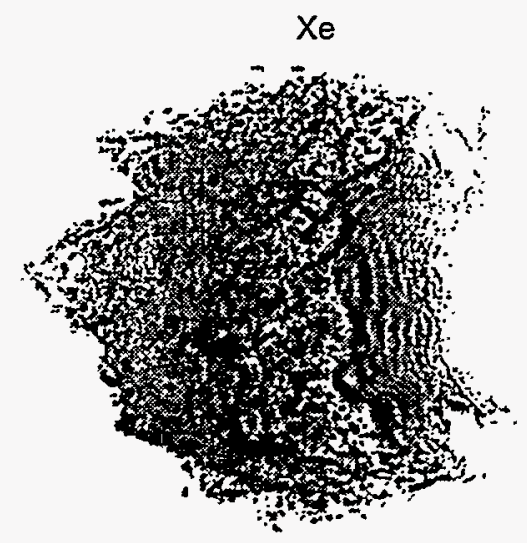

C)

Figure V-34. Experimental setup and data on the instability of a Taylor-Sedov shock. In A) the schematic of the experiment shows a 200-J laser incident on a $6-\mu \mathrm{m}$ thick plastic foil. The laser is $5 \mathrm{~ns}$ in duration and is focused to an $880-\mu \mathrm{m}$ diameter spot. The irradiated plastic will ablate and expand at greater than $10^{7} \mathrm{~cm} / \mathrm{s}$ into the surrounding fill gas. The gas fill is chosen to change the adiabatic index, $\gamma$, from 1.3 to $\sim 1.0$ for nitrogen and xenon fills, respectively. B) shows an optical dark-field shadowgraph of the shock wave at $243 \mathrm{~ns}$ in the nitrogen fill. The image has a frame time of $5 \mathrm{~ns}$ or less, and effectively freezes the shock front. The image shows that the shock front, the dark circle, is uniform and the material behind the shock is homogeneous. In C), the xenon fill shock front shows a completely different front, not uniform, with inhomogeneity in the material behind the shock. 
on the development of these secondary instabilities is, in itself, of great interest. Experiments with laser-driven flow will enable the compressible regime to be investigated, and this will have important implications for the understanding of wall-bounded shear flow.

Finally, a significant subject for investigation is radiative condensation instability, a classical astrophysical problem with applications to Tokamak plasmas and z-pinch plasmas as well. This instability is a general feature of the radiative cooling of an optically thin plasma. This is, of course, easily observed in interstellar and intergalactic clouds, solar prominences, and Tokamak or z-pinch plasmas, all of which have cool, dense plasma structures that radiate into hotter, rarefied plasmas in the surroundings. Experiments to provide a greater understanding of this instability are feasible in the controlled conditions characteristic of laser-generated plasmas.

\section{Turbulent Flow and Vortex Dynamics}

Both improvements in computational techniques and the emergence of chaos theory have contributed to a renewed interest in the subject of the transition to turbulent flow. In addition, the study of compressible turbulent shear layers has recently been the subject of increasing interest in the aeronautical engineering community. ${ }^{26}$

The understanding of compressible turbulent shear layers, which differs considerably from the more familiar transition to turbulence for incompressible flow, is of key importance in the field of scramjet engine design. An experimental program in this area would increase understanding of the applicability of the coherent structure models that have been developed for incompressible shear layers in the past. There is also a great need for experimental evidence of large-scale structure in compressible shear layers, comparable to that found in incompressible cases. It is for this reason that the larger spatial scales associated with NIF experiments could be important for this developing field of study.

Vortex dynamics ${ }^{27}$ are also an important part of this field, since they are associated with the fundamental mechanism for the evolution of hydrodynamically unstable surfaces to turbulence. The generation of vortices can be simulated in great detail, and the results of one such simulation are shown in Fig. V-35. The figure illustrates the results of a simulation of the generation of vortices in an incompressible flow, showing the density contours from a simulation of a planar wave. The wave is impinging on an interface that is at $60^{\circ}$ to the wave velocity. The case is the classically unstable case where the density of the medium of the initial wave is a factor of three smaller than the density of the shocked material. The Mach number of the shock

A) Initial position and time of 91

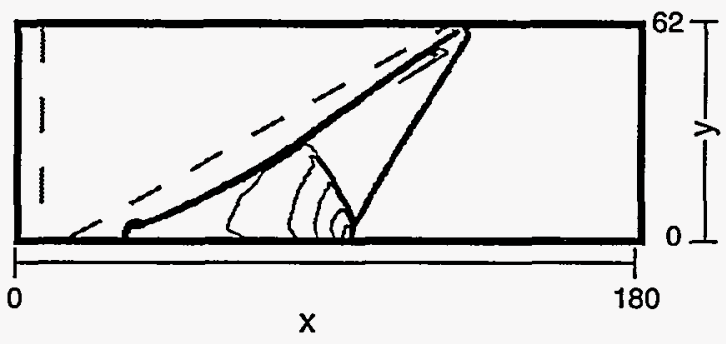

B) Late time of 620

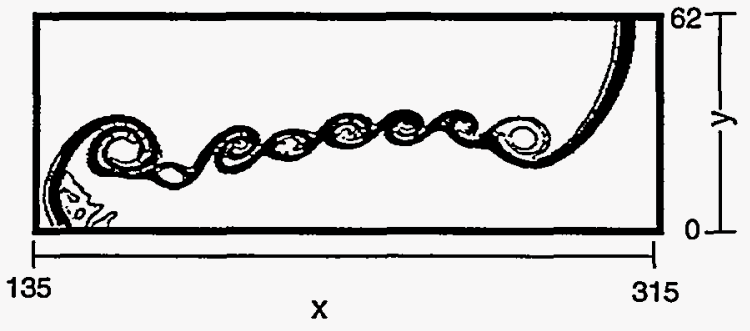

Figure V-35. The results of a simulation of the generation of vortices in an incompressible flow, showing the density contours from a simulation of a planar wave. The wave is impinging on an interface that is at $60^{\circ}$ to the wave velocity. In (A) the initial position of the wave and the interface are shown as gray dashed lines. Further, the scaled time for (A) is 91, when the shock has just passed the initial interface. Here the vortices are not yet formed. In (B), which is at a late time of 620 , the vortices are fully formed. At present these types of investigations are limited to incompressible flows; however, compressible-flow experiments will be possible with the large scales associated with NIF experiments. 
is 1.2 and the units of the calculations are scaled so that the sound speed in the initially moving medium is unity.

In (A) the initial position of the wave and the interface are shown as gray dashed lines. Further, the scaled time for $(A)$ is 91 , when the shock has just passed the initial interface. Here the vortices are not yet formed. In (B), which is at a late time of 620 , the vortices are fully formed.

At present these types of investigations are limited to incompressible flows; however, compressible-flow experiments will be possible with the large spatial scales associated with NIF experiments.

\section{E. References}

1. B. A. Hammel, D. Griswold, O. L. Landen, T. S. Perry, B. A. Remington, P. Miller, T. A. Peyser, J. D. Kilkenny, Phys. Fluids B 5, 2259 (1993).

2. P. M. Bell et al., in "Ultrahigh- and HighSpeed Photography" and "Videography," SPIE Proc. (SPIE, 1989), Vol. 1155.

3. B. A. Remington et al., Phys. Fluids B 4, 967 (1992).

4. B. A. Remington et al., Phys. Fluids B 5, 2587 (1992).

5. G. Dimonte and B. A. Remington, Phys. Rev. Lett. 70, 1806 (1993).

6. B. A. Hammel et al., JQSRT 51, No. 1 (1994).

7. See, for example, J. Bauche et al. in Advances in Atomic and Molecular Physics, D. Bates and B. Bederson, Eds. (Academic Press, 1988), Vol. 23, p. 131.

8. R. Cauble, D. Phillion, R. Lee, and T. Hoover, JQSRT 11, 433 (1994).

9. R. Elton, D. Billings, C. Manka, H. Griem, and B. Ripin, Phys. Rev. E 49, 1512 (1994).

10. C. Wu and P. Roberts, Phys. Rev. Lett. 70, 3424 (1993); Setchell, Storm, and Sturtevant, J. Fluid Mech. 56, 505 (1972).
11. M. Holt, Ann. Rev. Fluid Mech. 9, 187 (1977); M. Kamagai, Lawrence Livermore National Laboratory, Livermore, CA, UCRL-96675; J. Grove and R. Menikoff, J. Fluid Mech 219, 313 (1990).

12. B. Skews, J. Fluid Mech. 29, 705 (1967); L. Henderson, J. Fluid Mech. 26, 607 (1966) and 198, 365 (1989).

13. R. Klein, C. McKee, and P. Colella, Ap. J. 420, 213 (1994).

14. V. Kazakov, A. Legostaev, and S. Peigin, High Temperature 31, 717 (1993).

15. K. Holtsapple and R. Schmidt, J. GeoPhys. Res. 85, 7247 (1980), and 87, 1849 (1982).

16. G. Nutt and L. Klein, Phys. Fluids 24, 2143 (1981).

17. N. Byrne, SAIC; T. Geffen, SRI; and T. Peyser, Lawrence Livermore National Laboratory; private communication.

18. T. Afshar-rad et al., Phys. Rev. Lett. 73, 74 (1994).

19. T. Endo et al., Phys. Rev. Lett. 60, 1022 (1988).

20. R. Sigel et al., Phys. Rev. Lett. 65, 587 (1990).

21. B. A. Hammel, D. Griswold, O. L. Landen, T. S. Perry, B. A. Remington, P. Miller, T. A. Peyser, J. D. Kilkenny, Phys. Fluids B 5, 2259 (1993).

22. E. Muller, B. Fryxell, and D. Arnett, Astron. Astrophys. 251, 505 (1991).

23. G. Dimonte and B. Remington, Phys. Rev. Lett. 70, 1806 (1993).

24. J. Grun et al., Phys. Rev. Lett. 66, 2738 (1991).

25. K. Mikaelian, Phys. Fluids 6, 1943 (1994).

26. M. Zhuang et al., AALA J. 28, 1728 (1990); J. Hall et al., AIAA J. 31, 2247 (1993).

27. Hawley and Zabusky, Phys. Rev. Lett. 63, 1241 (1989); Zabusky et al., Phys. Rev. Lett. 67, 2469 (1991); P. G. Saffman, Vortex Dynamics (Cambridge University Press, New York, 1992). 


\section{Section VI}

\section{Material Properties}

\section{A. Equation of State}

Material pressures of millions of atmospheres (megabars or Mbar) to hundreds of millions of atmospheres are common in astrophysical objects, and are observed or predicted in the laboratory. Direct illumination of metals by a Nova-class terawatt laser can produce pressures of $100 \mathrm{Mbar}$ or greater. Gigabar (Gbar) pressures are predicted in spherically compressed capsules typical of inertial confinement fusion targets and radiatively driven samples. The thermodynamics and hydrodynamics of these systems cannot be predicted without a knowledge of the equation of state (EOS) for the high-pressure regime, because the EOS of a material largely describes how that material reacts to pressure.

Although the EOS of matter in the limiting case of extremely high pressure is expected to be described by a Thomas-Fermi model, the regime of applicability and approach to this limit are not known; EOS data is sparse at pressures above 3-4 Mbar because of the difficulty in producing high-pressure conditions while simultaneously measuring the relevant parameters. The ultimate goal is to use a material of known EOS as an EOS standard material to obtain EOS data on materials of interest.

In this type of experiment the shock velocity $u_{\mathrm{s}}$ and initial density $\rho_{0}$ are measured in each of two materials effectively adjacent to one another. Shock impedance is the product $\left(\rho_{0} u_{s}\right)$. If the EOS of material I is known, then it is possible to derive a Hugoniot point of material II (i.e., the locus of final states obtained from passage of shocks of varying strengths through the material) from the measured shock impedances.
At present there is no material with an EOS that is sufficiently accurate to be used as a standard at the pressures of interest. The EOS standards would be qualified by a series of experiments that use paired materials, both of which have equations of state currently under theoretical development in the pressure range of interest. The goal would be to provide accurate experimental data to test theory over a wide range of pressures, densities, and temperatures. The materials to be qualified as standards could be, for example, aluminum and copper, because the theory is under development and because of the relative ease of fabricating targets from these materials.

To perform this type of primary data experiment in the pressure range of $10-50 \mathrm{Mbar}$ (i.e., 1-5 TPa) requires, for direct illumination, intensities up to $\sim 3 \times 10^{15} \mathrm{~W} / \mathrm{cm}^{2}$, while for $\mathrm{x}$-ray drive, a temperature range of about $110-190 \mathrm{eV}$ will be required. To perform much higher pressure experiments would require a substantially larger laser system such as the NIF. This is a difficult regime, due not only to the accessible pressure regions, but also to the rigid constraints one must observe with respect to preheat, shock velocities measurements, and shock planarity.

There is exploration and development into three techniques that will allow us to obtain EOS data in the Mbar and near-Gbar regimes. All three use high-energy lasers as the driving source to produce intense shocks in materials. Indeed, laser deposition is presently the only way to produce these conditions in the laboratory. As discussed above, the shock brings the material into a high pressure state, albeit a transient one; 
measurements can then be made on the shocked matter to obtain EOS data points.

A primary objective of this work is to produce spatially large, planar shocks that are easy to diagnose and interpret. The shock must be spatially large enough that the data are unambiguous. Although very intense shocks can be generated by focusing a laser to a very small spot, these shocks generate side motion and potential development of plasma instabilities that require multi-dimensional modeling.

\section{Directly Driven High-Pressure Shocks}

The long history of attempts to produce very strong shocks in materials by direct irradiation with lasers has produced conditions inferred in targets of from 2 to $100 \mathrm{Mbar}$. However, these had problems with two-dimensional effects or extremely nonuniform spatial profiles of the laser, either of which can lead to laser-induced local hot spots and instabilities.

To ameliorate the laser nonuniformities, the laser spot has been smoothed with the use of a random phase plate. A random phase plate is a filter with a random pattern of small holes. It produces a far-field pattern that is even in intensity (within 10\%) except on scales smaller than about $10 \mu \mathrm{m}$. In addition, steering wedges have also been used. These steer different parts of the beam in the near field to different spots in the focal plane to produce a more flattopped distribution.

As an example, employing phase plates and steering wedges on a 1-ns beam of Nova provided an intensity of $3 \times 10^{14} \mathrm{~W} / \mathrm{cm}^{2}$ of $2 \omega_{0}$ light in a focal spot that was more than $1 \mathrm{~mm}$ in diameter and had a uniformity of $\pm 10 \%$ over the spot diameter. The beam was focused on a $25-\mu \mathrm{m}$ thick aluminum disk, and the rear side of the disk (i.e., the side away from the beam) was imaged with a UV streak camera. The result was an extremely uniform shock breaking out of the rear of the target.

Figure VI-1 shows a schematic of the setup for the experiment. The streak image in Fig. VI-2 shows that the shock, after traversing the $25-\mu \mathrm{m}$ thickness of aluminum in approximately $650 \mathrm{ps,}$ varies in breakout time across the entire

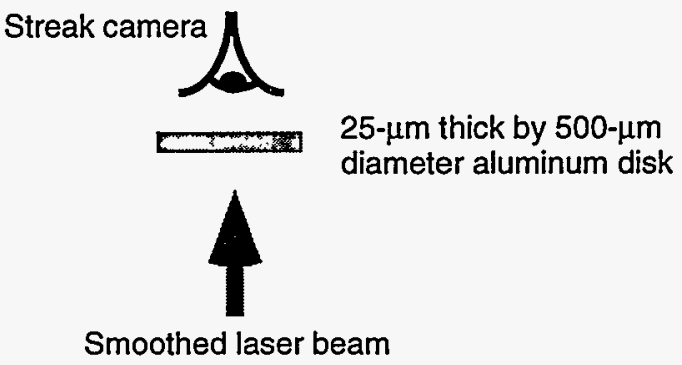

Figure VI-1. Schematic of direct laser-driven shock experiment. A spatially smoothed beam of Nova irradiates an aluminum disk, $25 \mu \mathrm{m}$ thick by $500 \mu \mathrm{m}$ in diameter. The schematic also shows the position of the streak camera (streaked UV imager).

diameter by less than $20 \mathrm{ps}$. This implies that the shock was planar across the disk by better than $1^{\circ} .1$ Pressures inferred in the shocked aluminum, using stepped targets and wedges, were 20-30 Mbar.

\section{Indirectly Driven Colliding Foil Experiments}

To reach a regime of much higher pressure without sacrificing spot size, and thus onedimensionality, a very different technique has been employed-a variation and miniaturization of the well-known flyer-plate technique. ${ }^{2}$ In this method, the flyer (a foil in the present case) stores kinetic energy from the driver over an acceleration time and delivers it much more rapidly as thermal energy in collision with another foil. In addition, the flyer acts as a preheat shield so that the target remains on a lower adiabat than if it were exposed to the driver. These attributes make it possible to achieve much higher pressures using a flyerimpact foil or an indirectly driven colliding foil than when using a directly driven configuration.

In the experiments, an $\mathrm{x}$-ray drive was produced by focusing the ten beams of the Nova laser into a millimeter-scale cylindrical gold hohlraum and utilizing the radiation escaping from a hole in the cylinder. The experimental arrangement is illustrated in Fig. VI-3. The 


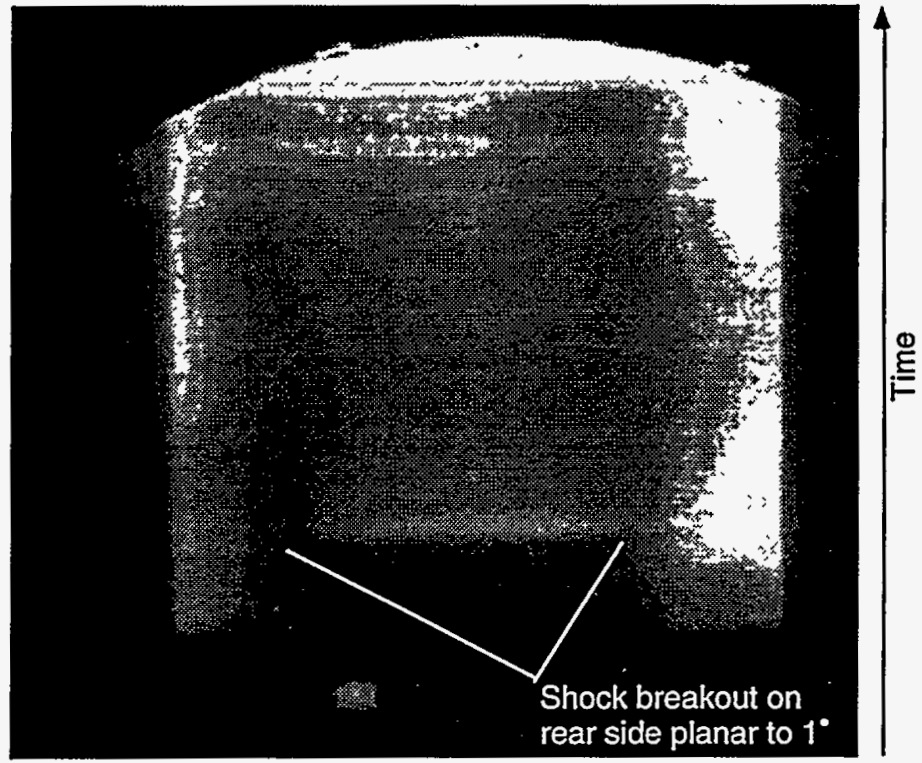

Figure VI-2. Streak image of direct laser-driven shock experiment. Image is from the diagnostic, showing an extremely planar shock emerging from the rear side of the target.

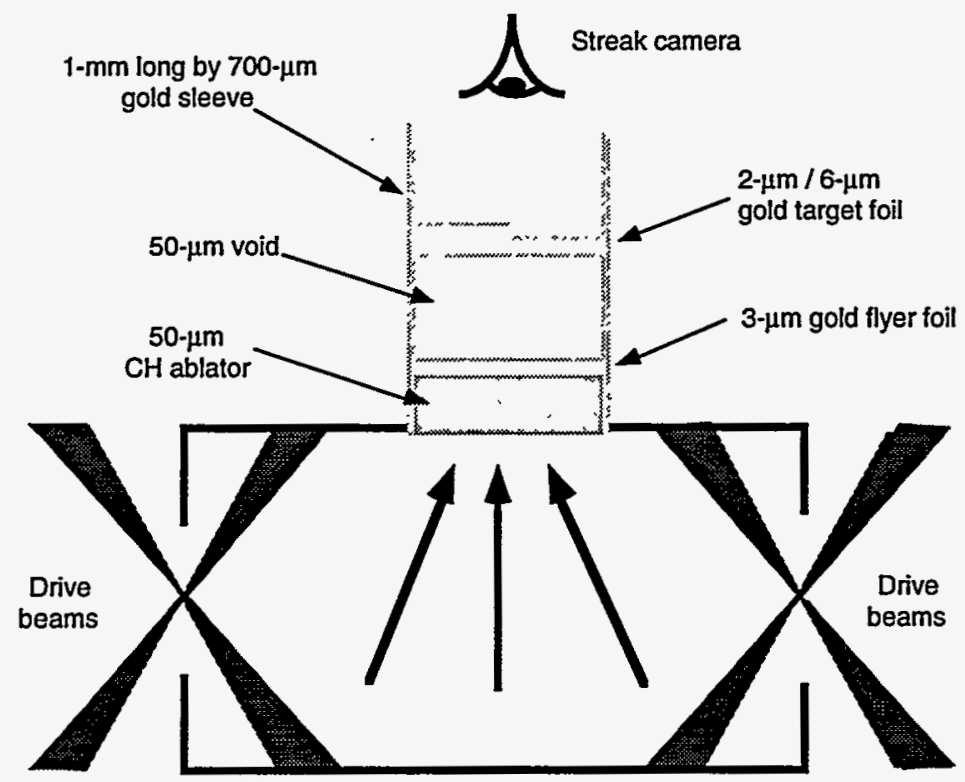

Figure VI-3. Schematic of radiation-driven shock using a 2-step target foil. The diagram shows the colliding foil arrangement (not to scale). The 1-mm-long by $700-\mu$ m-diameter experimental package is shown facing the hohlraum $x$-ray drive. Components of the package are a $50-\mu \mathrm{m}$ plastic ablator to which is attached a $3-\mu \mathrm{m}$ gold flyer foil, a $50-\mu \mathrm{m}$ void, and a two-thickness gold target foil, all held in a gold sleeve. 
hohlraum x-rays ablated a 50- $\mu \mathrm{m}$ layer of polystyrene to which was attached a 3- $\mu$ m-thick gold foil. This flyer foil accelerated through a $50-\mu \mathrm{m}$ void region and, near the end of the laser pulse, collided with a stationary gold target foil composed of two thicknesses $(2 \mu \mathrm{m}$ and $6 \mu \mathrm{m}$ ), launching a compression wave into the target foil. The shock on the rear side of the target foil was imaged with an optical streak camera.

The experimental packages were more than $0.5 \mathrm{~mm}$ in diameter, and the cylindrical target assembly was mounted across a hole in the wall of the hohlraum with the ablator facing the hohlraum interior. The approximately $100-\mu \mathrm{m}$ long target assembly was placed at the bottom of a $1-\mathrm{mm}$ gold sleeve so that the assembly was completely shielded from unfocused, unconverted laser light. Additional shielding prevented the streak camera from viewing heated areas of the sleeve and the hohlraum.

Fig. VI-4 shows a typical streak camera image. The image shows shock breakout at two times corresponding to the two thicknesses of the target foil; the time interval between the breakout times measures the shock speed in the target, assuming the shock speed is constant. Here, the interval measured between breakout times on the two thicknesses is $57 \pm 5 \mathrm{ps}$, corresponding to an average shock velocity of $70 \pm 6 \mathrm{~km} / \mathrm{sec}$. From the SESAME equation-of-state tables, this shock speed corresponds to a density of $90 \mathrm{~g} / \mathrm{cm}^{3}$ and a pressure of 0.74 Gbar in the gold target, by far the highest inferred pressure obtained in the laboratory.

Any slight spatial imbalance in the drive or any unpredicted edge effects (from interactions between the flyer foil and the sleeve, for example) could cause the flyer to tilt or curve, which would drive a non-planar shock into the target.

However, the relatively large diameter of the foils would allow any non-planarity in shock breakout to be observed. In addition, the step in the target was at the center of the large foil, where the effects of edge-induced nonuniformities would be minimized.

If the target foil were preheated by highenergy $x$-rays from the hohlraum before the flyertarget collision, the measurement would be compromised. To test for this possibility, the $x$-ray drive was altered in one experiment so that overall drive intensity was identical to the other experiments, but the intensity of high-energy $x$-rays (those $\geq 2.5 \mathrm{keV}$ ) was reduced by

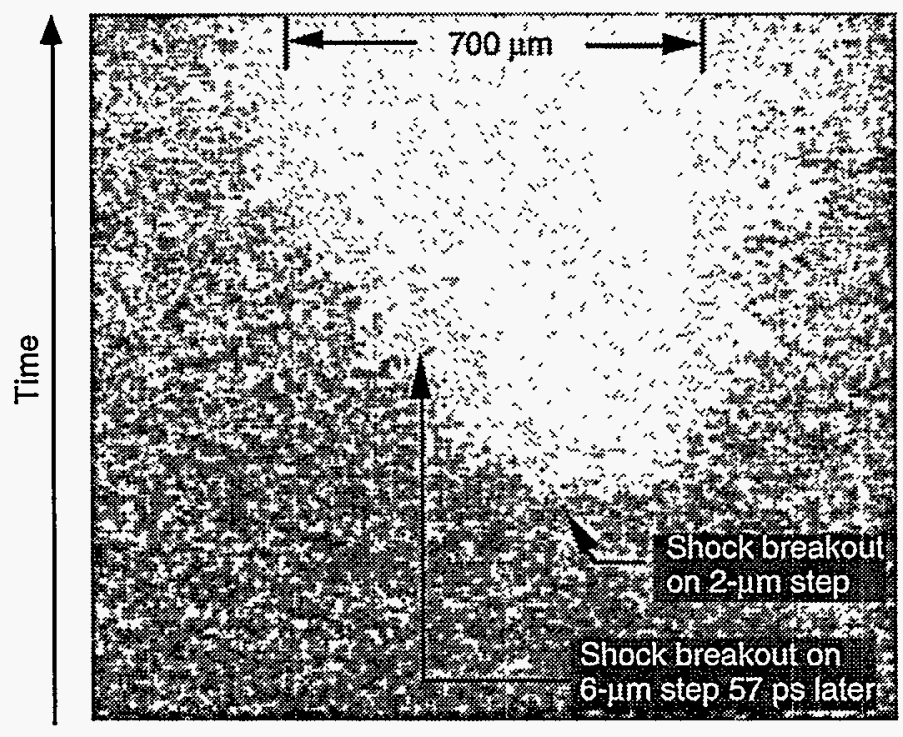

Figure VI-4. Streak camera image of radiation-driven shock using a 2-step target. Image shows shock breakout on the rear surface of the two-step target foil at two distinct times corresponding to the two thicknesses of the target. This time interval provides the shock travel time across the $4-\mu \mathrm{m}$ step difference. The interval in this case is about $57 \mathrm{ps}$, indicating a shock speed of $70 \mathrm{~km} / \mathrm{sec}$. 
more than a factor of five. The result was well within the uncertainties of the other experiments, so the results are not compromised by preheat.

A straightforward extension of this technique can be made to obtain EOS data for this regime. If the flyer foil can be shielded so that the flyer does not significantly heat or decompress, EOS data points can be found by measuring the speed of the flyer foil. Since it has been demonstrated that the target foils do stay intact, this can be accomplished by modifying the target foil by shielding it, as shown in Fig. VI-5.

With the target foil shielded and stepped, shock speed and flyer speed can be measured simultaneously. (The speed of the material behind the shock can be inferred from the flyer speed.) These two measurements will allow the calculation of an EOS primary data point in the Gbar regime. Thus, in addition to the shock speed discussed in reference to the single-stepped target shown in Fig. VI-4, the flyer speed can be simultaneously obtained by recording shock breakouts from two identical foils placed at different distances along the flyer path. One experiment was performed with a three-step gold target foil. Because the condition of the flyer foil was unknown, an EOS data point could not be evaluated, but observed

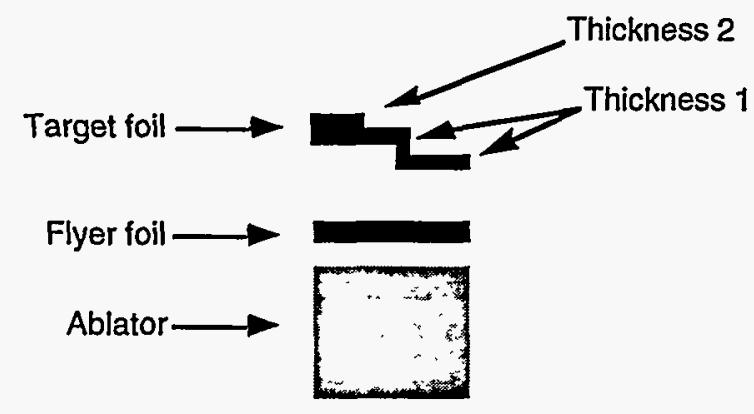

Figure VI-5. Schematic setup with target foil shielded and stepped to allow simultaneous measurement of shock speed and flyer speed. (From the flyer speed, the speed of the material behind the shock can be inferred.) These two measurements will allow the calculation of an EOS primary data point in the Gbar regime. shock breakout from the three steps indicates the viability of the approach.

To proceed with EOS measurements, the condition of the flyer foil must be known; indeed the accuracy of the result may depend on the flyer remaining solid. The development of a highmagnification $x$-ray laser radiograph technique to determine the conditions of accelerated foils has been undertaken and this is described in the section on $x$-ray laser applications in Section VIII, Radiation Sources.

\section{Indirectly Driven Shock Experiments on Plastic}

Plastics are very different materials than metals, but they are just as pervasive in highenergy laser experiments as major constituents of diverse targets. Since plastics, unlike metals, are largely transparent to high-energy $x$-rays, $x$-rays can be used to backlight relatively thick samples of plastic and provide information on the sample as a function of time.

In particular, the shock front in a sample of plastic can be seen on a streak camera by simultaneously imaging the transmission of an $x$-ray backlighter through both the shocked and the unshocked material. Transmission through the denser, shocked plastic is significantly less than through normal-density plastic-that is, the transmission is about $70 \%$ less for a compression of 4 in a $0.7-\mathrm{mm}$ thick sample using a $7-\mathrm{keV}$ backlighter energy. If, at the same time, motion of the material behind the shock can also be imaged, a data point in the EOS is obtained.

An experimental arrangement that accomplishes this, adapted from an experiment to measure material mix at interfaces, ${ }^{3}$ is depicted in Fig. VI-6. The material motion behind the shock is viewed as the difference in transmission of the backlighter through shocked, doped plastic and shocked, undoped plastic; this is the interface shown in the figure. The drive, an $x$-ray source, is provided by a hole in the side of a gold hohlraum.

Here only eight beams of Nova are used for the drive, saving two beams for the backlighter. Code simulations predict a pressure of $\leq 40 \mathrm{Mbar}$ near the interface. Two experiments have been 


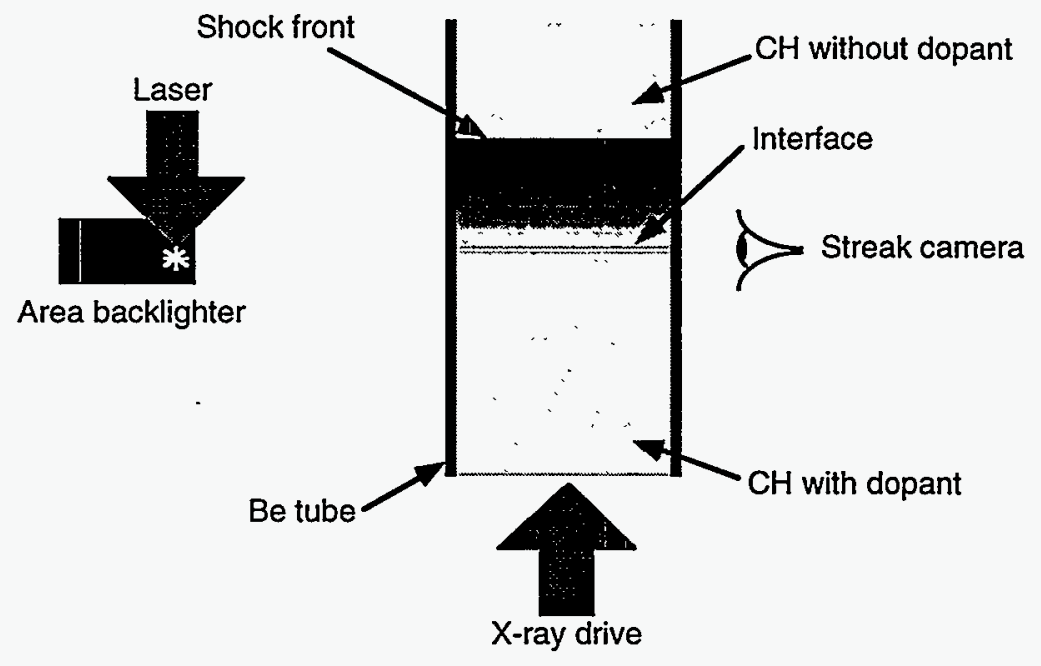

Figure VI-6. Overview of arrangement for radiation-driven shock experiments in transmissive media such as plastic $(\mathrm{CH})$, showing relative positions of the package (which is mounted across a hohlraum), backlighter, and streak camera. The drive, an x-ray source, is provided by a hole in the side of a gold hohlraum. Differences in transmission of the backlighter through the package allow measurement of both the shock speed and the speed of the interface between doped and undoped plastic. This allows direct measurement of the equation of state of plastic in the multi-Mbar regime.

performed, both with $2 \%$ bromine doped into the first section, which was $300 \mu \mathrm{m}$ long. A 3-ns long backlight beam was initiated at about the time the shock was expected to pass through the interface. Although the interface was clearly visible on the streak records, the shock front was too weak to be seen. However, this technique will allow EOS data to be obtained on plastic in the multi-Mbar regime.

\section{B. Opacity}

Knowledge of the $x$-ray opacities ( $x$-ray absorption) of hot matter is essential for understanding its state and radiative transport. The Nova laser has been used to obtain experimental, high-quality opacity measurements. This not only required the development of high-resolution spectroscopic techniques to measure the $x$-ray transmission of the opacity samples, but also required the development of techniques to accurately and simultaneously measure the temperature and density of the samples. Simply, to provide a benchmark of the ability to measure a local thermodynamic equilibrium (LTE) opacity, measurements must be performed on relatively simple atomic species first, so that the technique can be evaluated with some degree of faith in the predictions. This requires exacting procedures that provide the tempera-ture, density, and gradient scale lengths on a single experiment.

The $\mathrm{x}$-ray absorption of opacity samples was measured by the method of point projection spectroscopy, which is illustrated in Fig. VI-7. In this experiment, eight of the laser beams at Nova are used to heat the opacity sample. Then, a point source of $x$-rays is produced by tightly focusing one of the remaining laser beams onto a small backlight target of high- $Z$ material. $X$-rays from the backlight pass through the opacity sample onto an $x$-ray diffraction crystal and are then recorded on $x$-ray film. Other $x$-rays from the same point backlight bypass the sample, but are still diffracted from the crystal onto the film record.

The ratio of the $x$-ray spectrum attenuated by the sample to the unattenuated $x$-ray spectrum provides the $x$-ray transmission spectrum of the sample. Proper collimation 


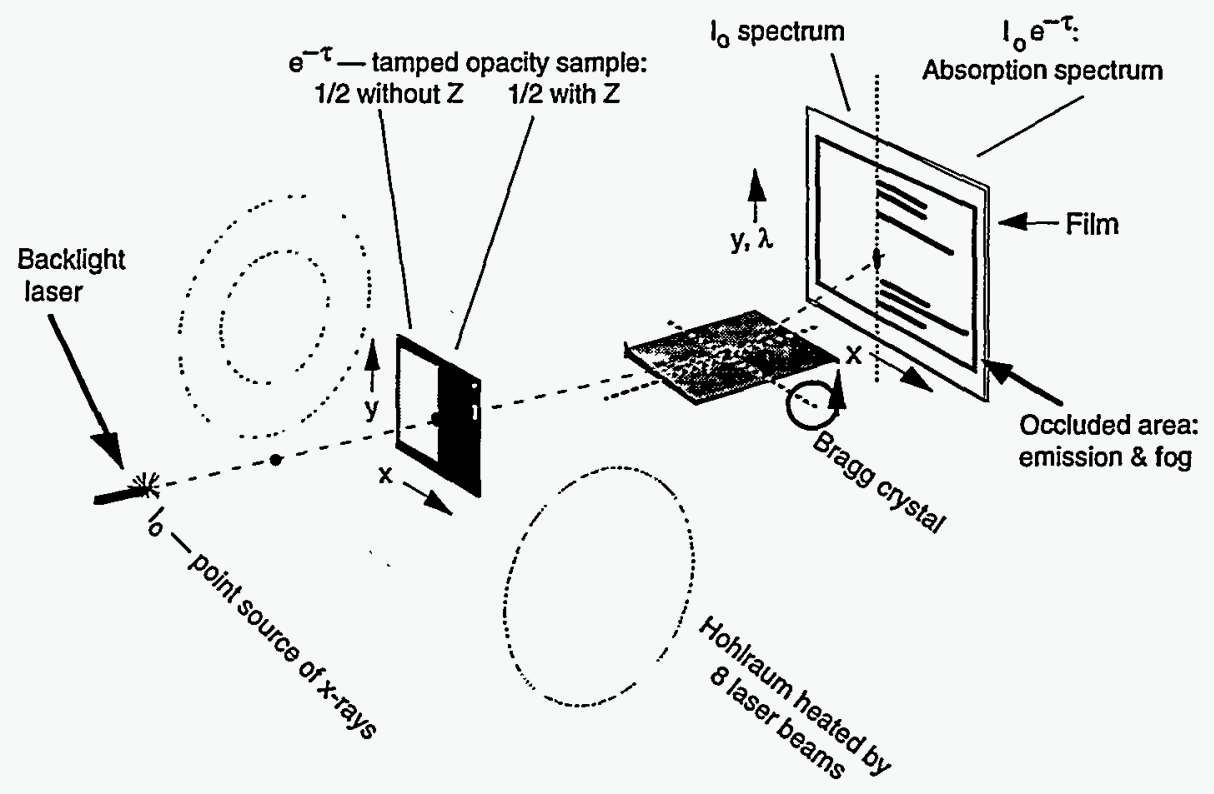

Figure VI-7. Schematic of point projection spectroscopy method for measuring opacity. The laserproduced backlight $x$-rays pass through the target (the opacity sample) onto an $x$-ray diffraction crystal and are then imaged on $x$-ray film. Because the crystal disperses the spectrum, the result is an image that is both spatially and spectrally resolved. Temporal resolution is provided by backlight duration.

allows a highly quantitative analysis of the spectrum. Backgrounds from film chemical fog, sample emission, and crystal $x$-ray fluorescence can all be separately determined from the $x$-ray film record.

Careful attention was paid to the sample conditions. It was required that the sample be uniform throughout in both temperature and density. Uniformity of temperature was attained by heating the sample in a special hohlraum. The hohlraum was constructed so that no laser light impinged on the sample either directly or on first reflection; thus, the sample was heated only by $x$-rays. The hohlraum also kept a uniform temperature throughout the sample, and this, along with the relatively high density of the sample, ensured that the sample was in local thermodynamic equilibrium.

Further, the samples were tamped by plastic so that the density throughout the sample was constant as the sample expanded. The thickness of the tamper was determined by hydrodynamic calculations, and density uniformity was checked in the experiments. This experimental technique allowed the measurement of the opacity of aluminum and laid the basis for using aluminum opacity as an independent temperature diagnostic.

In further experiments, the density of the samples was determined by imaging the samples. This was done by using a second point-projection spectrometer to image the expansion of the samples. This spectroscopic record was also used to verify that the samples had uniform density throughout the sample. The temperature of the sample was determined by mixing the sample with aluminum. A third pointprojection spectrometer was used to measure the absorption of the aluminum $n=1$ to $n=2$ transitions. The relative intensities of the transitions from the different ion species gave the ion balance in the aluminum which (coupled to the density measurement) gave the temperature of the sample.

These techniques allowed density to be measured to an accuracy of $\pm 20 \%$ and temperature to an accuracy of $\pm 3 \%$. With these accuracies it was possible to make quantitative comparison between the experimental results and the theoretical calculations of opacity. ${ }^{4}$ 
In particular, one experiment measured the opacity of niobium. The niobium sample contained $14 \%$ aluminum by weight for the temperature measurement described above. Figure VI-8 shows the transmission of the aluminum (upper) and the niobium (lower). The dashed lines overlaying the experimental data are the calculations. In general there is excellent agreement.
This experimental result defines a milestone in that we have proven the ability to measure the opacity of the aluminum sample with sufficient accuracy that it can now serve as an in-situ temperature diagnostic for the sample. The accuracy of the temperature, measured to be $48 \mathrm{eV}( \pm 2 \mathrm{eV})$, indicates an important advance on previous attempts to measure temperatures of high-energy-density matter.
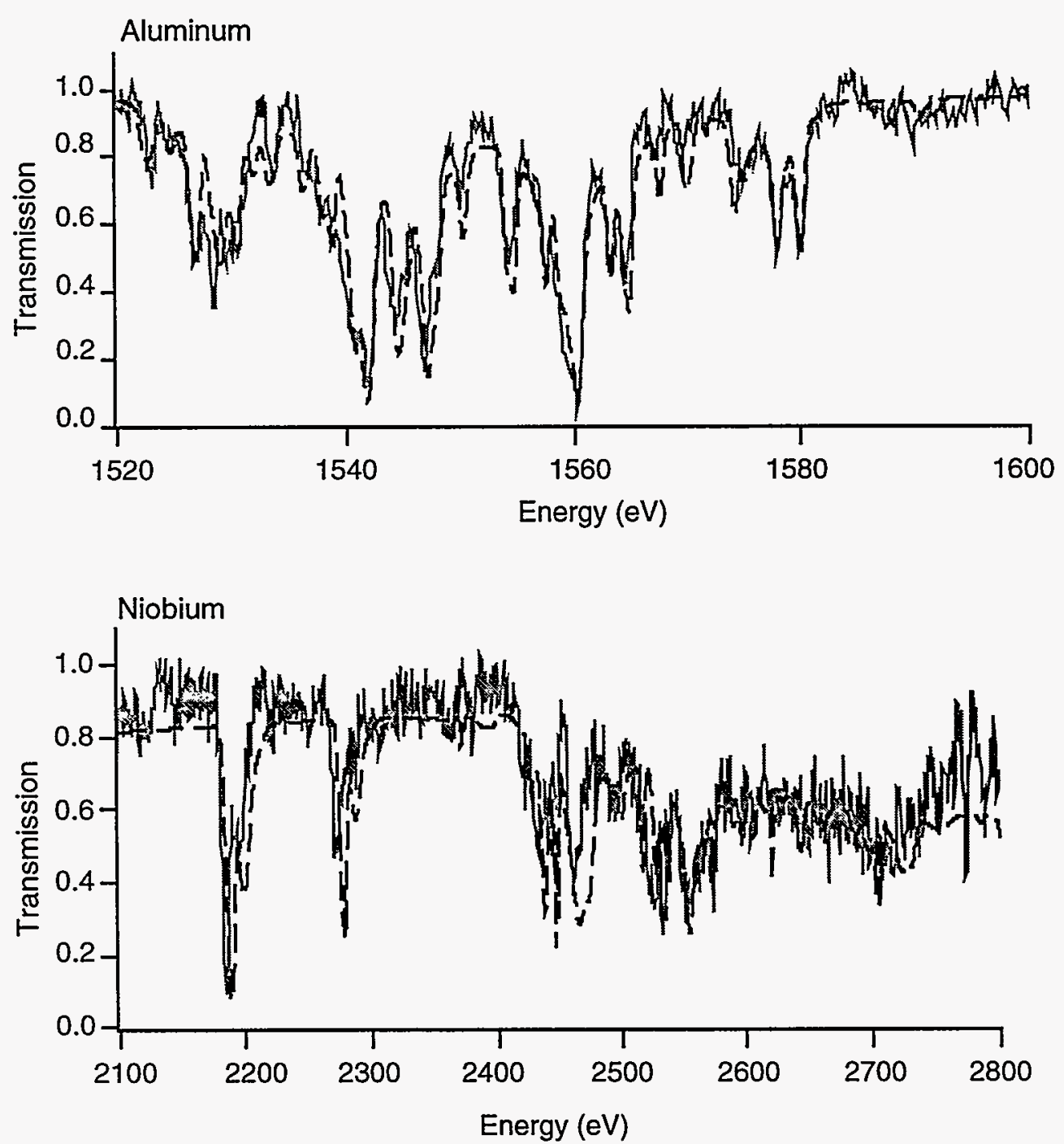

Figure VI-8. Absorption (opacity) of the aluminum/niobium sample. The experimental data are shown as a solid gray line and the LTE opacity prediction calculated using OPAL shown as a dashed line. The upper graph shows the transmission of aluminum, the lower graph shows the transmission of niobium. The spectrum of the aluminum $K \alpha$ lines, which have been previously verified to yield an accurate temperature, are measured on the same experiment as in the niobium spectrum in the lower graph. 
Other experiments on opacity have confirmed the astrophysically important iron $\Delta \mathrm{n}=0, \mathrm{n}=3$ absorption feature, which causes a large increase in opacity above older predictions. ${ }^{5}$ The work on iron has been further extended to measurement of the spectrum in sufficient detail to evaluate the Rosseland mean for comparison with theoretical predictions. ${ }^{6}$ Both of these studies on iron opacity are motivated by astrophysical considerations and indicate that, in at least this one area, the experimental capability has become mature.

\section{Strength of Material}

Laser generation of multi-kilobar shocks has several applications to the study of high explosives, high-velocity impacts, and the alteration of the mechanical properties of certain alloys and ceramics. There have been several important advances in the field since the early 1970s. Some of the most common methods of studying such shock waves include interferometric methods and the use of piezoelectric transducer gauges. However, these methods yield little information on the dynamics of the near-front face, where the shock wave is initiated.

It is possible to measure shock strength (in terms of compression ratio) and spatial density profile using an $\mathrm{x}$-ray diffraction technique for studying laser-induced shocks within various crystalline materials. This can be used for pressures from $1 \mathrm{kbar}$ up to approximately $0.5 \mathrm{Mbar}$, where shock melting occurs. This $\mathrm{x}$-ray diffraction technique is ideally suited to observation of the dynamics of the front surface of laser-shocked materials. The technique can make a significant contribution to our understanding of shock launching, and because it is a direct measurement of the relevant parameters of the near-front surface, it can enable conclusive investigations of the response of novel materials. It is also possible to extend this technique to probing of the rear-surface breakout of the shocked material.

The technique is based on the fact that when a crystal is subject to strain, its $\mathrm{x}$-ray diffraction properties are altered-most notably the $\mathrm{x}$-ray diffraction peak of the crystal is broadened. The physical reasons for this are easy to understand: a strained crystal essentially has a distribution of interatomic spacings at which the Bragg condition can be satisfied, and therefore the shape of the diffraction peak is a function of the spatial strain profile. This fact has been used to study strain profiles in crystals where the strain is caused by dopants or by laser annealing. (The transient alteration of the diffraction peak in a laser-shocked crystal has also been suggested as a means of obtaining subnanosecond $x$-ray switching.)

The experiment to measure compression in the crystal proceeds as follows (see Fig. VI-9). A shock is launched into the crystal of interest. At some point during the shock launching a short (100-ps) pulse of $x$-ray line radiation is produced by a synchronous, but delayed, laser beam irradiating a separate target. This pulse is Braggdiffracted off the front surface of the shockcompressed crystal. The compression of the crystal changes the Bragg condition according to the formula

$$
\Delta \mathrm{d} / \mathrm{d}=-\operatorname{Cot} \theta \Delta \theta \text {. }
$$

Thus, the line radiation is diffracted to a higher angle than that for the unperturbed crystal, and this angular shift directly indicates the change in the interatomic spacing within the shocked region. Because there will be a density gradient at the crystal surface, there will be, in addition to the overall shift, a range of angles at which diffraction takes place. Thus, information can be obtained about the density gradient as well as about peak density. Indeed, it is possible to extract a density profile from the data contained in the diffracted pulse.

The primary interest in this technique would be to find a method of studying the materials under extreme conditions. The present set of known experiments is restricted to simple structures with small heated areas. To develop larger perturbed areas and study the materials at greater depth would require a substantial effort in the development of larger lasers and $x$-ray sources for probing. 

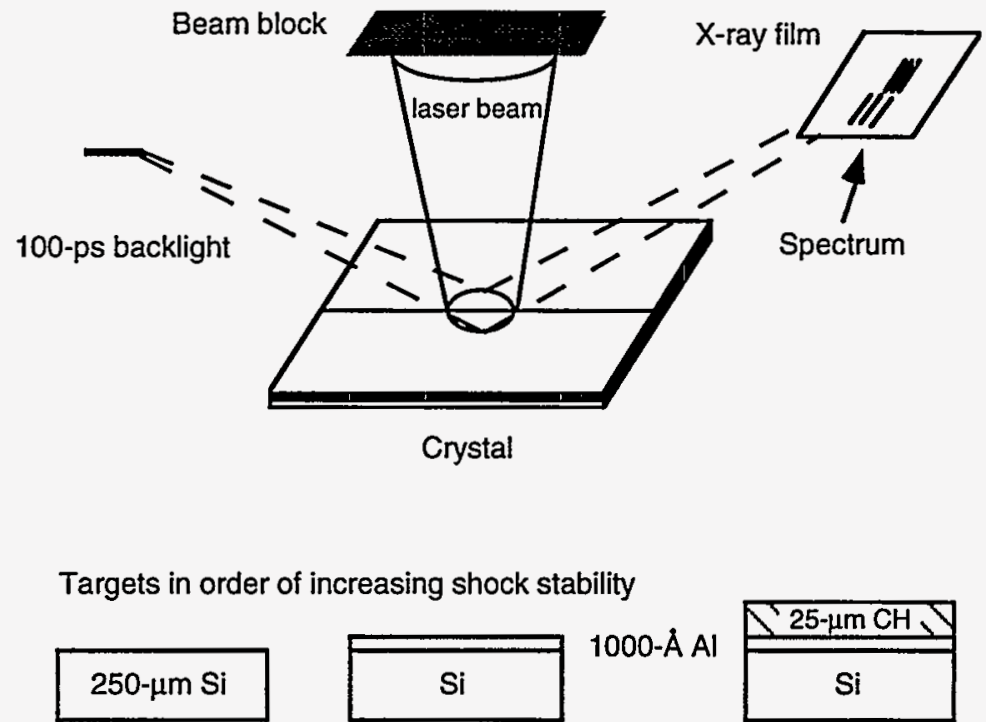

Figure VI-9. Schematic of $x$-ray diffraction technique used to study the dynamic structure of a crystal. The crystal is compressed using a laser-induced shock from a monochromatic $x$-ray source. During the shock transit through the crystal, a short (100-ps) backlight pulse is Bragg-diffracted off the front surface of the shock-compressed crystal, and the resulting spectrum is recorded on the $x$-ray film.

\section{Simple Compression Studies}

Silicon has been shocked and transient strains of up to $10 \%$ have been observed. The method of Bragg diffraction relies on the fact that the crystal remains "single"; i.e., all the planes running parallel to the shock front are compressed uniaxially and the periodicity of the crystal as a whole is maintained. ${ }^{7}$ In some ways it is quite surprising that such an ordered state exists so far above the Hugoniot elastic limit, which for silicon in the (111) direction corresponds to an elastic compression of about $2.6 \%$. Thus, one simple experiment is the investigation of the maximum observable compression for a series of different crystalline materials, and comparison of this with shock melting data obtained by more conventional means.

It is interesting to note the utility of highenergy lasers for the following two reasons. First, they provide for a relatively high shock strength within the material of interest ( 1 Mbar). Although smaller lasers could attain local pressure of this magnitude, it could only do so over a spot size on the order of a square millimeter. A large spot is needed so that the shocked region of the crystal subtends a large range in angles to the $x$-ray source.

Second, they provide harder $x$-rays. Small lasers may not have sufficient backlight energy to produce hard enough $x$-rays for accurate diagnosis. It has been found that at large compressions, such as the $10 \%$ compressions in silicon, the $\Delta \Theta$ becomes large for softer $X$-rays. Soft $x$-rays diffracted from the shocked portion of the crystal get smeared out on the x-ray filming, making diagnosis difficult. The change in Bragg angle for harder $\mathrm{x}$-rays is not so great, facilitating diagnosis. For example, for the $10 \%$ compressions in silicon, the helium-like titanium resonance line was an appropriate $x$-ray source. Much harder $x$-rays may be required to measure compressions that are close to shock melting $(30-40 \%)$ and for investigating crystals with shorter interatomic spacings, such as lithium fluoride and quartz.

\section{Plasticity and Adiabatic Shear Bands}

Other questions that should be experimentally ripe include investigating the behavior of a single crystal on the lattice level when it is shocked above the Hugoniot elastic 
limit, and determining how long the crystal takes to start to deform plastically. 8

There is evidence that some materials, when shocked above the Hugoniot elastic limit, form small bands of micron or sub-micron dimension where the dislocation density is extremely high; such areas are known as adiabatic shear bands. Evidence for such localized yielding is mainly based on recovery experiments rather than on insitu observation.

It is of interest to attempt to observe these bands by a short-pulse $x$-ray topography experiment, using a point source of laser-plasmaproduced $x$-rays. Point sources of $5 \mu \mathrm{m}$ dimensions have routinely been produced using lasers, and these could be used to image shear bands. Although the source size is larger than the width of the bands, the bands can run several microns into the crystal, and imaging should be possible in the same way that dislocations of Angstrom size can be imaged using conventional $x$-ray topography.

It is also of interest to determine the shape of the unit cell as the crystal is compressed above the Hugoniot elastic limit. This can be done by using simultaneous Bragg and Laue diffraction with thin crystals. Bragg diffraction takes place from the surface of the crystal, the surface being parallel to the shock front, and measures the elastic compression. Observing the Laue diffraction through the crystal at the same time, interrogating the planes running perpendicular to the shock front, provides information about the plastic component of strain.

Experiments have been performed using $50-\mu$ m-thick silicon (111) crystals, with Laue diffraction off a (220) plane through the crystal. Although both Bragg and Laue lines were observed when no shock was present, the intensity of the Laue line was not sufficient to enable diagnosis when the crystal was compressed. Improvements in crystal preparation and an increase in backlight energy should permit measurements of this type.

\section{Metastable Phases}

It is believed that many crystals, when shocked above the phase change point, proceed to a metastable phase before completion. One example is potassium chloride, which undergoes a phase change from sodium chloride structure to cesium chloride structure at a pressure of about $20 \mathrm{kbar}$. Investigations of the multiple wave structure in sodium chloride have been interpreted as transformation to a metastable phase within $2 \mathrm{~ns}$, with completion to the cesium chloride structure taking between 25 and $500 \mathrm{ns,}$ depending on crystal orientation.

Investigations of potassium chloride have been performed, and it was found that the diffraction lines from the sodium chloride phase disappeared upon shocking. However, the exact strain at which the disappearance occurred was not determined. Time-resolved $x$-ray diffraction from the shocked crystal could permit measurement of the strain at which diffraction disappeared, which is expected to be at the point where the phase change occurs. Thus, one would simply "streak" the diffraction.

Disappearance of the diffraction record suggests that the new phase is polycrystalline, as would be expected in an order-disorder transition. Thus, the ability to obtain diffraction information from powders would be of importance.

\section{Phase-Change Measurements}

The first, and only, in-situ $x$-ray diffraction evidence for a shock-induced phase transition is that obtained from shocked boron nitride, which transforms to a wurtzite structure. It is a displacive transition without a change in unit cell volume or occupancy. Such transitions should take place on an inverse phonon time; i.e., a picosecond time scale. Obviously it would be interesting to repeat and extend these measurements with the improved time resolution and synchronization available with high-power laser technology.

In addition to shocking boron nitride, it would be interesting to investigate $\mathrm{TeO}_{2}$. This material undergoes a transition from tetragonal to orthorhombic at a pressure of $9 \mathrm{kbar}$. This is interesting because the transition proceeds by an acoustic shear wave phonon traveling along (110) at an unusually slow sound speed of about $1 \mu \mathrm{m}$ per ns. 


\section{Rear-Surface Studies}

Dynamic tension can be produced within the body of a material by subjecting the front surface of a planar target to impulsive loading. Tensile strain is then induced when the duration of the pressure pulse, $\tau$, is less than $c d$, where $c$ is the shock velocity within the material and $d$ its thickness. In this case two rarefaction waves cross; one of them produced at the front surface as the pressure loading falls off, and the other produced at the rear surface as the incident compression wave is reflected. For sufficiently high tensile stresses, a section of the rear portion of the target separates, or "spalls," from the bulk.

At laser irradiances $>10^{9} \mathrm{~W} / \mathrm{cm}^{2}$, absorption of the laser light produces a high-pressure plasma at the target surface. As this plasma expands away from the surface, momentum balance launches a compression wave into the bulk of the target. The temporal profile of the compression wave is similar to that of the laser pulse.

Direct measurements of tensile elastic strain in silicon (111) wafers have been obtained at strain rates above $10^{8} \mathrm{~s}^{-1}$. Tensile elastic strains of $3.4 \%$ have been directly observed by in-situ picosecond $x$-ray diffraction. ${ }^{9}$ This may constitute a novel method of addressing the ideal strengths of materials.

Figure VI-10 shows a schematic of a rearsurface shock breakout experiment to probe the shock structure of a material using a monochromatic $x$-ray source. Prior to the breakout, the probed rear surface will be in compression, during the breakout there will also be tension due to a rarefaction wave, and finally the rear surface will go into tension with no compression.

The experimental results are shown in Fig. VI-11, where the $x$-ray streak camera record shows the entire history of the rear surface from unshocked, to the initiation of the breakout, through to the time when the crystal is in pure tension. From Fig. VI-11 it can be seen that at early times, for the first $\sim 200$ ps, the $x$-rays are probing the crystal in a state of com-pression, with the angular shift in diffraction corresponding to a maximum compression of $6.2 \pm 0.2 \%$. Later the $x$-rays are being diffracted from both regions of compression and regions of tension, and finally, at late times the x-rays are being diffracted from a region of pure tension, with a maximum observed tension of $3.4 \pm 0.2 \%$.

These three regimes correspond to the times when the reflected wave has not yet collided with the rarefaction wave due to release of pressure at the front surface; the time during collision of the rarefaction waves; and the time when the reflected wave has passed the front surface rarefaction wave by at least the probe depth of the $x$-rays.

Figure VI-12 compares the spectra from a shocked crystal with those from an unshocked crystal at various stages of an experiment (Fig. VI-11 shows similar stages in a streak camera record). Thus, within the target the strain has changed from $6.2 \%$ compression to $3.4 \%$ tension within $\sim 600 \mathrm{ps}$ (i.e., at a strain rate of $1.6 \times 10^{8} \mathrm{~s}^{-1}$ ). The maximum observed tensile strain $3.4 \%$ corresponds to a uniaxial tensile stress of approximately $70 \mathrm{kbar}$, comparable to the largest fracture stresses observed in static measurements.

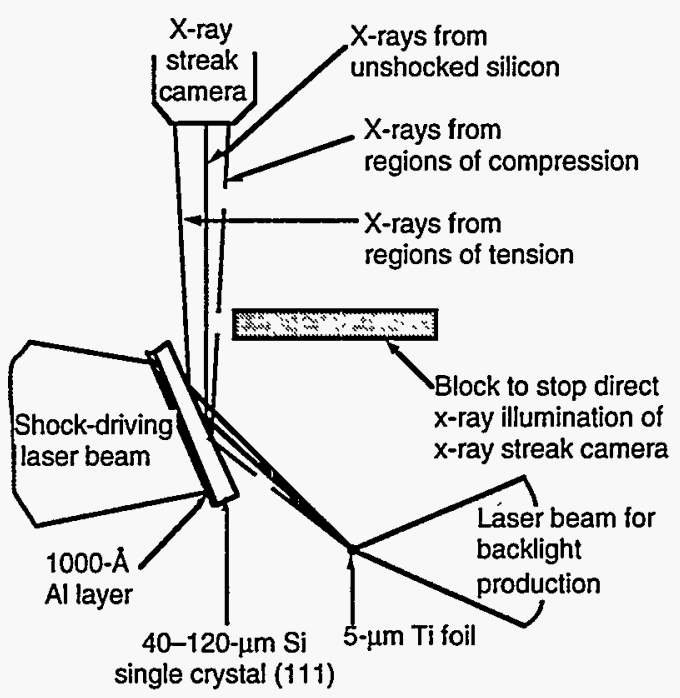

Figure VI-10. Schematic of a rear-surface shock breakout experiment to probe the shock structure of a material using a monochromatic $x$-ray source. Prior to the breakout, the probed rear surface will be in compression, during the breakout there will also be tension due to a rarefaction wave, and finally the rear surface will go into pure tension with no compression. 


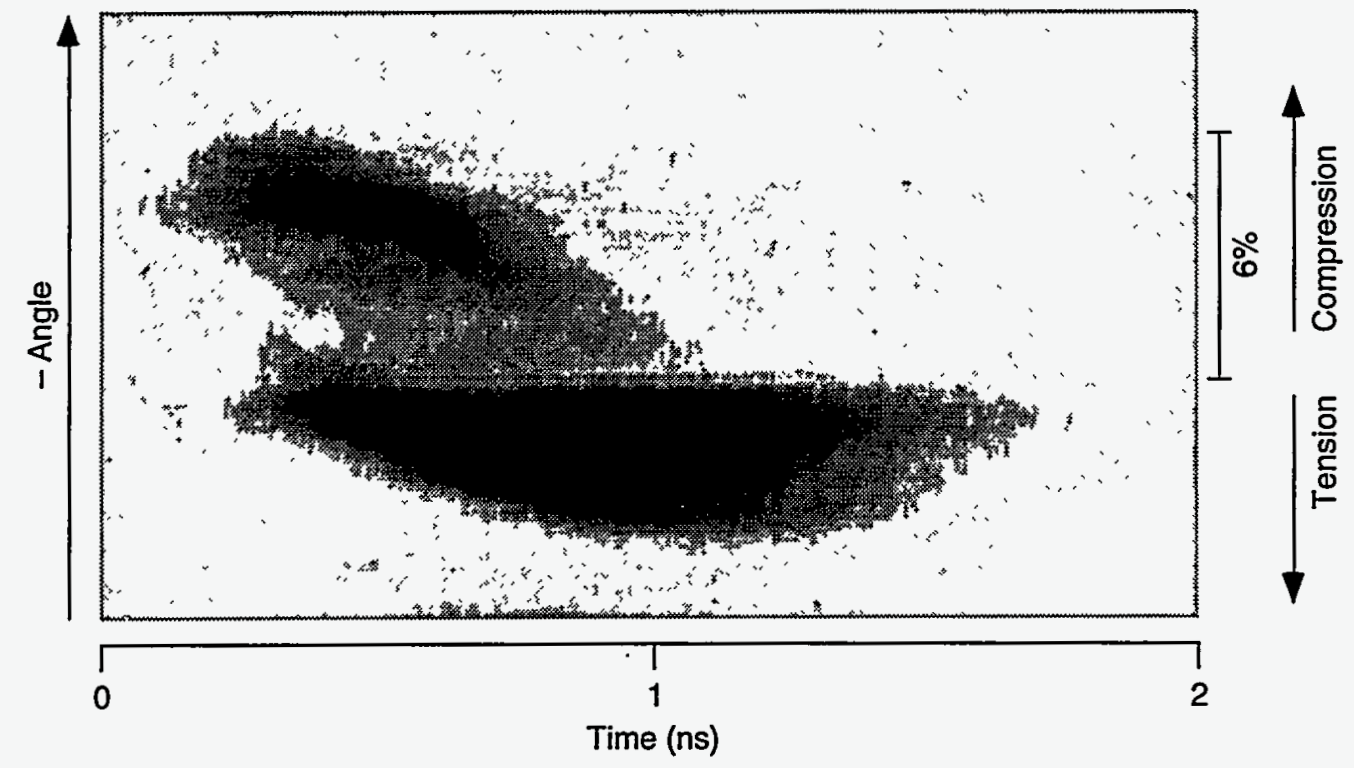

Figure VI-11. Data from the rear-surface shock breakout experiment in Fig. VI-10, showing a shock transiting through the material. The streak camera record shows the entire history of the rear surface, including the compression phase at early time as the shock nears the rear surface, the compression and tension after the shock breaks out, and the late-time phase of pure tension.

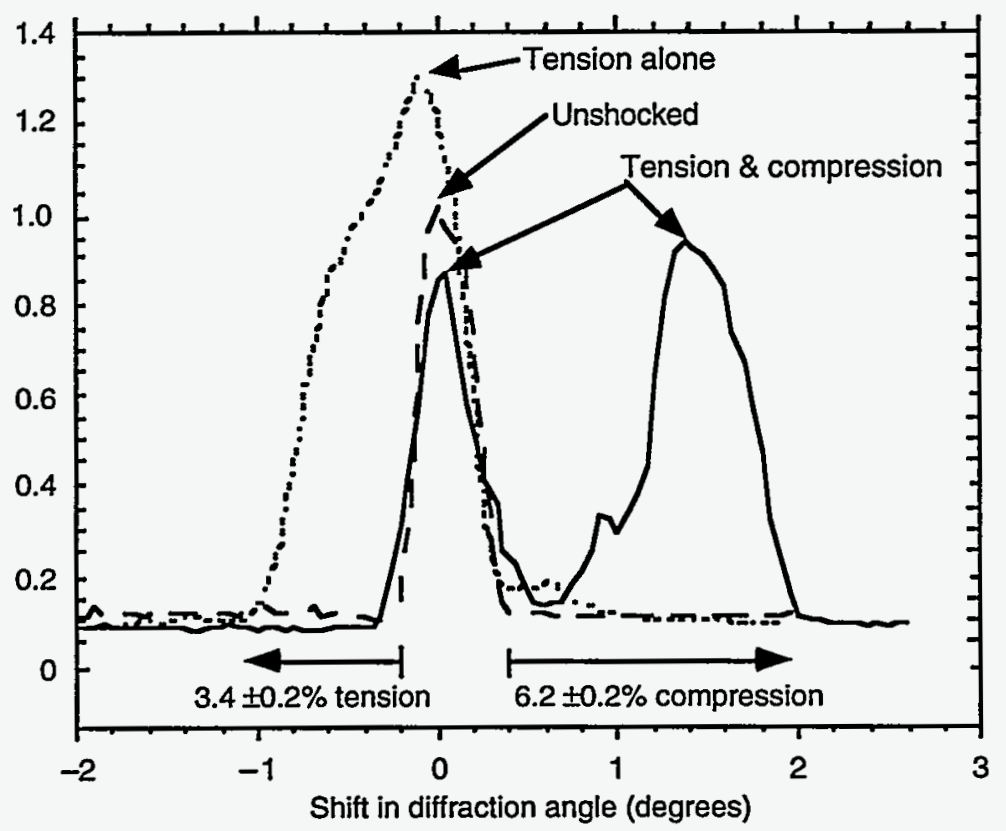

Figure VI-12. Spectra from the probed shocked rear surface of a crystal. The stages shown graphically here are comparable to the stages visible in the streak camera record in Fig. VI-11. The three generic periods are shown - the pure unperturbed (unshocked) crystal in a dashed line; the period of shock breakout, when there is tension and compression, in a solid line; and the pure tension late-time phase in the dotted line. 
Finally, it is of interest to point out that with the use of a powder diffraction camera and a bright source (very bright indeed), one should be able to extend all these studies to non-crystalline materials. This would open the way for a new source of studies of the detailed responses of a much wider range of materials.

\section{Future NIF Experiments}

The study of the properties of materials at extreme conditions of pressure, temperature, and density has been a goal of laser experiments since the advent of high-energy facilities. The most common application has been in investigations of equations of state (EOSs) - the relationship between pressure, density, and internal energy.

Impacts using guns or high explosives to generate shock waves have provided nearly all of our current database in this area, starting with investigations during World War II. Those methods have provided a great deal of precise data on a wide range of materials, but are limited to pressures below $1 \mathrm{TPa} .{ }^{10}$ Static compression experiments have been performed extensively in diamond-anvil high-pressure cells; these experiments will ultimately be limited to pressures below roughly $1 \mathrm{TPa}$ by the insulatormetal transition of diamond. Higher pressure experiments have been carried out at pressures from $1 \mathrm{TPa}$ to over $100 \mathrm{TPa}$ in the vicinity of nuclear explosions, but the data are of low precision above $10 \mathrm{TPa} .{ }^{11}$

Relevant to the present discussion we note that high-intensity lasers can achieve similar high pressures, as was recently demonstrated. ${ }^{12}$ In the higher pressure regime, many new phenomena will become apparent, such as pres-sure ionization, strongly correlated fluid states, and so on. The strain rates involved can range up to $10^{20}$ $\mathrm{bar} / \mathrm{s}$, and the behavior of materials under these conditions is unknown, especially as applied to melting and other changes of phase.

Figure VI-13 is a plot of stress vs strain rate domain. It shows the regions accessible to various experimental methods. High-stress, highstrain-rate tensile states can be attained in solids when a shock breaks out of a free interface of a sample. Experimental fracture strengths of brittle materials show a $1 / 3$ power dependence with the tensile loading strain-rate. Extrapolating these fracture stress measure-ments, which were taken at strain rates below $10^{5} \mathrm{~s}^{-1}$, to strain rates up to $10^{10} \mathrm{~s}^{-1}$, tensile stress equal to the theoretically predicted tensile strengths can be reached.

Strain rates as high as $10^{9} \mathrm{~s}^{-1}$ are already possible using high-power lasers and in-situ $x$-ray diffraction. With NIF-like technology, the peak tensile stresses will be far in excess of those presently realizable and will be at ultrahigh strain rates. This capability will make it possible to investigate the fracture properties and ultimate strengths of many materials, including novel ceramics.

We have essentially no bulk or atomic-scale knowledge of any materials under these conditions of pressure loading. Precise measurements are necessary to discriminate among competing models or theories. The NIF is important in that it is the first large laser of sufficient energy and pulse length to allow for the possibility of such measurements being made with sufficient accuracy.

Data and validated theoretical models are vital for understanding and modeling such diverse phenomena as inertial confinement fusion, planetary interiors, and ultrahigh-velocity meteorite impacts. Data and validated theoretical models are also vital for most of the scientific experiments to be performed at the NIF, especially hydrodynamics and radiative and transport properties in condensed phase systems at high pressure $(\mathrm{P})$ and temperature $(\mathrm{T})$. We need to know the properties of materials over wide ranges of pressure and temperature. The detailed sections that follow describe the problems and some proposed experiments to determine the EOSs for:

- The principal Hugoniot.

- Multiple shock states.

- Isentropic release states.

- Isochoric heating experiments.

- X-ray diffraction studies.

- Other material properties.

In doing so, we will describe applications to other fields. 


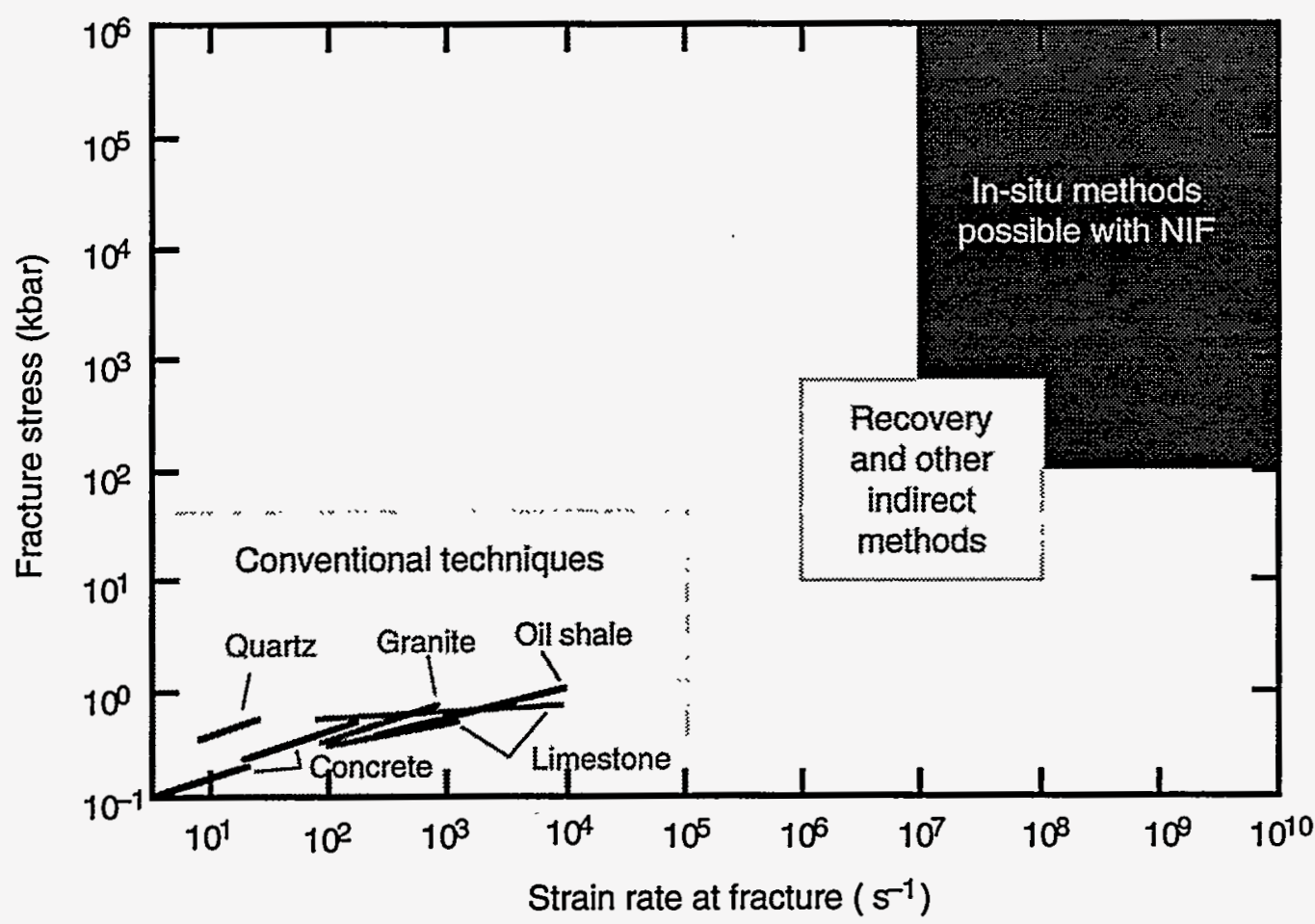

Figure VI-13. Plot of stress vs strain rate domain, showing the regions accessible to various experimental methods. High-stress, high-strain-rate tensile states can be attained in solids when a shock breaks out of a free interface of a sample. NIF technology will make it possible to investigate the fracture properties and ultimate strengths of many materials, including novel ceramics.

\section{The Principal Hugoniot}

The principal Hugoniot is the locus of single shock states for shocks of varying strength, starting from material at normal density, atmospheric pressure, and $300^{\circ} \mathrm{K}$. These are not thermodynamic paths, but a true locus of states (see Figure VI-14).

The Rankine-Hugoniot relations, ${ }^{13}$ which express conservation of mass, momentum, and energy across the shock front, make the principal Hugoniot particularly useful. The final shock state (pressure, density, and total energy) can be determined from a knowledge of the initial state and two dynamic variables. The two dynamic variables are

- The velocity at which the shock propagates through the undisturbed medium, referred to as either $u_{s}$ or $D$.

- The velocity of the material behind the shock front, referred to as either $u_{p}$ or $U$ and known as the mass or particle velocity.
For steady shocks, $u_{s}$ is directly accessible to measurement, in principle.

Shock compression of both fluids and solids can be visualized as a discontinuous change in state variables. This assumption is used in derivation of the Hugoniot-Rankine relations. With this view only the initial and final states of a shock-compressed material exist. In Fig. VI-14 we see that for an initial state (point $A$ ), there exists an infinite number of end states. A specific end state is reached when a material is subjected to a shock of specific strength. By performing a number of experiments, each with a different shock strength, a locus of end points is mapped out (points B, $B^{\prime}$, and $B^{\prime \prime}$ ). This locus of end points is known as the Hugoniot.

The straight lines joining the initial condition $A$ and each of the end points $B$ in the PV plane are the Rayleigh lines. The slope of a Rayleigh is proportional to the shock velocity-higher strength compressions are associated with faster shock velocities. 
Figure VI-15 illustrates the relationships of the Hugoniot, an isotherm, and an isentrope all going through the initial point $A$. A single point on the Hugoniot, which is the locus of end states, is reached after shock compression. Shock compression involves an increase in entropy, and the Hugoniot will always lie above the isentrope. Because any increase in entropy is associated with a rise in temperature, the Hugoniot also lies well above the isotherm. However, there may be situations where this is not true (for example, during a phase change) and in these cases a shock is thermodynamically impossible. With the capability of the NIF to

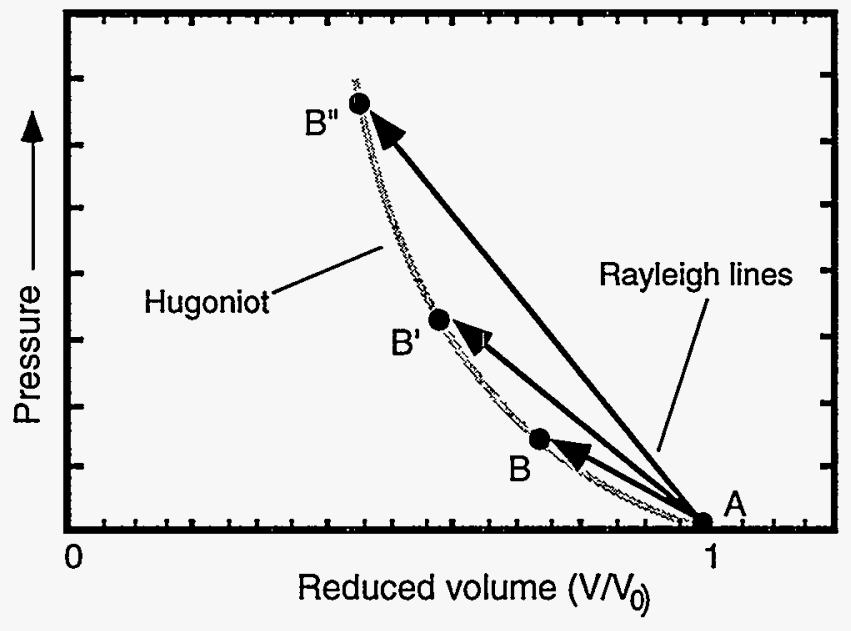

Figure VI-14. The principal Hugoniot defined. Shock compression of both fluids and solids can be visualized as a discontinuous change in state variables. For an initial state (point $A$ ), there exists an infinite number of end states. A specific end state is reached when a material is subjected to a shock of specific strength. Points B, B', and B" represent a locus of end points from experiments with different shock strengths. This locus of end points is known as the Hugoniot. The straight lines joining the initial condition A and each of the end points B in the PV plane are the Rayleigh lines.

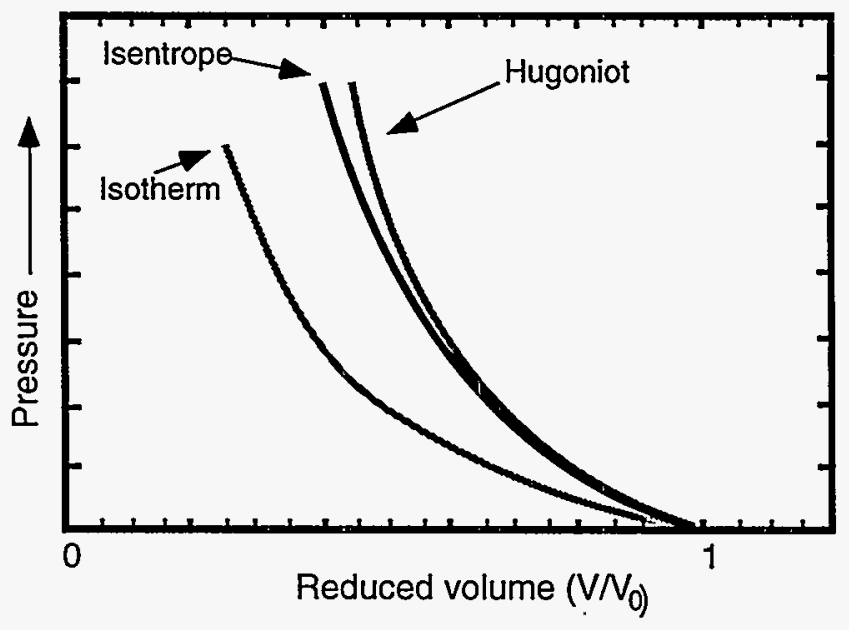

Figure VI-15. Relationships of the Hugoniot, an isotherm, and an isentrope all going through the same initial point. Shock compression involves an increase in entropy, and the Hugoniot will always lie above the isentrope. Because any increase in entropy is associated with a rise in temperature, the Hugoniot also lies well above the isotherm. 
provide more energy and long tailored pulses, yielding large scale-lengths and increased planarity, several of the current problems in achieving measurements will be overcome. This will open new horizons by permitting uniformity and constancy of shocks over time scales relevant to shock studies.

The standard method is to measure the transit time $\Delta t$ across a step of known extent $\Delta x$, and then $u_{s}=\Delta x / \Delta t$. Although this is a straightforward concept, there are several practical difficulties in current experiments, difficulties the NIF can alleviate.

First, it is assumed that the velocity is constant. This has been rather difficult to achieve in ablatively driven shocks. With careful target design, the long, temporally tailored pulse of the NIF can solve this problem.

Second, the velocities are large-for aluminum at $10 \mathrm{TPa}, u_{s} \approx 70 \mathrm{~km} / \mathrm{s}$. Such a shock wave will cross a $100-\mu \mathrm{m}$ step in only $1.4 \mathrm{~ns}$, and the $1 \%$ accuracy we desire implies an uncertainty in the measurement of only $14 \mathrm{ps}$. This will strain the ability of current diagnostic systems.

The NIF will allow greater spatial dimensions, which will allow higher fractional accuracy for measurements of both length and time. Further, the use of a hohlraum drive will help ensure that the shocks are planar and of large transverse extent, which is necessary if the larger target samples are to be free of twodimensional effects. Accurate measurements are a requirement, because the uncertainty in shocked density varies roughly as the product of the compression with the uncertainty in shock velocity, and compressions greater than four will be common.

It is, in principle, possible to determine compression directly using $\mathrm{x}$-ray backlighting; in practice, we believe that to be extremely difficult. What remains is to measure the mass velocity. Again, the scale of targets posited for the NIF makes possible a method proposed years ago, but never realized. The idea is to directly measure the velocity of the shocked material by observing the motion of an interface in the shocked material using $x$-ray sidelighting with an $x$-ray streak camera. The velocities of the shocked material are large as well, but the spatial dimensions over which the measurement can be made are improved at the NIF by virtue of the relatively long time scale possible for the experiment. For it to become possible to directly measuring the velocity of the shocked material, a pointbacklighter beam is essential to avoid parallax effects.

A further possible set of experiments to produce information on the principal Hugoniot is the use of what have become known as impedance-match experiments. In these experiments the equation of state of an unknown material is compared to that of a known material-perhaps by the methods described above. ${ }^{14}$

This method has been used in laser experiments before, but the scale of the experiments was such that only limited accuracy was possible. ${ }^{15}$ The method can be exploited at the NIF because of the planarity and uniformity of the drive in hohlraums. One suggestion is that a simple material such as aluminum or gold be developed as a "standard" material over the broadest pressure range on the principal Hugoniot, and then used as a point of comparison with other materials of interest.

On the principal Hugoniot, there are a number of materials that must be studied. Hydrogen is the chief component of the Jovian planets. The current upper limit on single shock experiments in the fluid is $0.02 \mathrm{TPa},{ }^{16}$ which samples only a fraction of the Jovian interior. Solid $\mathrm{D}_{2}$ or $\mathrm{H}_{2}$ could be shocked to many $\mathrm{TPa}$ in well-characterized experiments, perhaps encountering the proposed plasma phase transition. 17

Further, metals of various $\mathrm{Zs}$ need to be studied. For aluminum in the range $10-100 \mathrm{TPa}$, successive pressure ionization steps are predicted to occur, and they may lead to wide excursions of the density on the Hugoniot as compared to calculations with purely statistical models. ${ }^{18}$ In low- $Z$ metals such as beryllium, these excursions are expected to occur below $10 \mathrm{TPa}$.

The Rankine-Hugoniot conditions relate the dynamic variables and initial state to yield the total shock energy, and do not explicitly give the temperature of the shocked medium. This is an important issue, since temperature, rather than 
total energy, is often an explicit variable in theoretical models. When we have recourse to competing models in the range of a few tens of $\mathrm{TPa}$ (several hundred megabars), we find that at a given pressure, the densities vary by about $30 \%$, while temperatures are known to no better than a factor of two.

This is no mere academic problem, since the problem of high-pressure turbulent hydrodynamic flows explicitly involves temperature and entropy, and understanding these flows may prove intractable without more detailed information about the distribution of total energy into external and internal degrees of freedom. The solution to this problem may be to determine temperature by $\mathrm{x}$-ray absorption spectroscopy, using absorption line ratios to determine the temperature of the shocked fluid. We note that the value of temperature measurements is not limited to the principal Hugoniot; these comments apply to other highpressure thermodynamic states and processes as well.

\section{Multiple Shock States}

The principal Hugoniot is a single locus of states; we need to know the properties of materials over wider ranges of pressure, especially planetary or other isentropes. This region can be probed by the use of multipleshock experiments. Of this type of experiment, we can distinguish two main possibilities.

The first is colliding shocks, initially traveling in opposite directions. This method can be used to generate extremely high pressures, since relative velocity is doubled, causing pressure to be increased by a factor of about four.

A view of the multiple shock trajectories in the PV space together with an idealized schematic of a multiple shock experiment is shown in Fig. VI-16. For single-shock compression there is a limiting compression of fluids and solids which is asymptotically reached at high pressures. For large increases in pressure, small compressions are achieved at the expense of significant material heating.

Multiple shocking of a material allows greater compressions at lower temperatures and pressure to be attained. In Fig. VI-16, for example, a material is shocked from its initial state $A$ to state $B$ on the principal Hugoniot. If the material is shocked again while in state $B$, the new end state $C$ will lie on another Hugoniot that is defined by the new set of initial conditions $B$. Again a limiting compression is defined that is some fraction of the specific volume of state $B$.

The schematic in Fig. VI-17 shows a doubleshock compression experiment, with a particle at a position $A$ embedded in the material to be shocked. In the top picture, a piston drives a shock (small arrow) from the right into the compressible material (solid). In the middle picture, the shock, traveling toward the wall, passes the particle at point $A$. As the shock passes it, the particle moves from state $A$ to state $B$. In this change of state it acquires a particle velocity that is towards the wall in direction, but its velocity is lower than the shock velocity. The shock hits the immovable

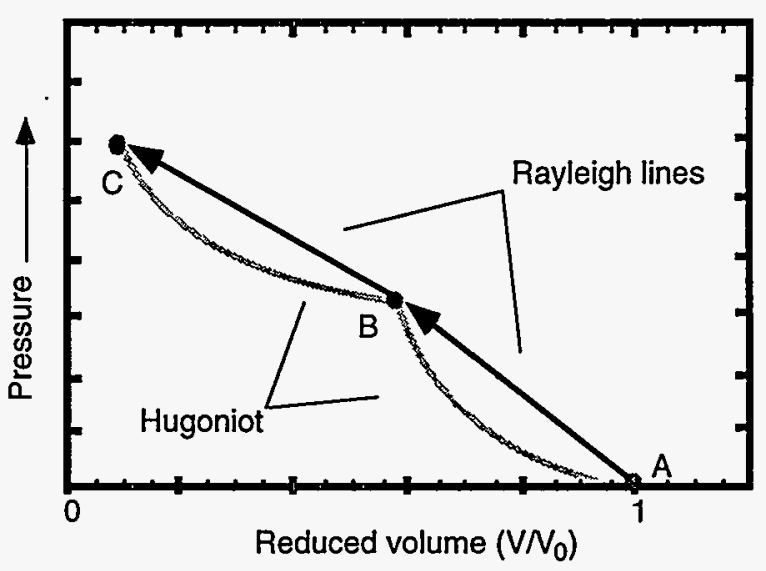

Figure VI-16. Double-shock compression experiment. Multiple shocks allow access to a wide range of conditions, making it possible to attain greater compressions at lower temperatures and pressure. In the figure, a material is shocked from its initial state $A$ to state $B$ on the principal Hugoniot. If the material is shocked again while in state $B$, the new end state $C$ will lie on another Hugoniot that is defined by the new set of initial conditions $B$. 


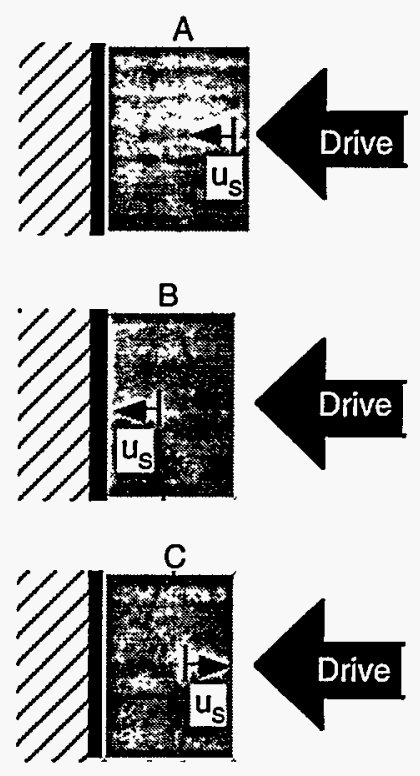

Figure VI-17. Schematic of double-shock compression experiments. A particle at a position $A$ is embedded in the solid material to be shocked-a compressible material (solid) adjacent to an infinite-impedance, immovable wall (heavy black line). In the top picture, a piston drives a shock (small arrow) from the right into the compressible material. As the shock (middle picture), traveling toward the wall, passes the particle at point $A$, the particle moves from state $A$ to state $B$. In this change of state it acquires a particle velocity that is towards the wall in direction, but its velocity is lower than the shock velocity. The shock hits the immovable wall and is reflected into the solid, now traveling toward the right (bottom picture). The shock is now moving back into the compressed material, which is under pressure (from the piston), has been heated, and has a velocity-this is state $B$. As the shock passes it the second time, the particle moves to state $C$ on a second Hugoniot, acquiring a new particle velocity.

wall and is reflected into the solid, now traveling toward the right (bottom picture). The shock is now moving back into the compressed material, which is under pressure (from the piston), has been heated, and has a velocity-this is state $B$. As the shock passes it the second time, the particle moves to state $C$ on a second Hugoniot, acquiring a new particle velocity.
Situations akin to this occur naturally in solids-for example as a solid is shockcompressed beyond the Hugoniot elastic limit (in the weak shock regime), or during a phase transition. The flexible pulse technology of the NIF will enable advanced methods of obtaining multiple shock states.

In the case of relatively oblique incidence, we have the opportunity to study shock interactions that may lead to hydrodynamic instabilities, since there will be a shear component to the flow. This type of experiment is only practical with the use of large high-energy lasers, because the problems of timing and balanced shock generation are intractable for other drivers. The topic of instabilities is touched upon in Section V, Hydrodynamics.

Of greater interest is the possibility of multiple or reverberating shocks in a material initially at rest. For a given final pressure, multiple shocks will achieve higher final densities and lower temperatures. With multiple shocks, ten-fold compressions can be achieved.

The limit of very weak multiple shocks approximates adiabatic compression; all multiple-shock states of this type fall between the principal Hugoniot and the $0^{\circ} \mathrm{K}$ isentrope. This is an important regime for planetary physics, because all of the major planets are believed to have interior distributions of temperature and pressure that are best approximated by isentropes. As we noted in the introduction, current technology can sample only a very small fraction of those interiors. Using liquid or frozen hydrogen targets, experiments on the NIF will be able to probe states that are typical of the deep interiors of the Jovian planets, as well as of "brown" dwarfs in general.

Multiple shocks can be generated by reflecting a simple shock wave at a boundary of high shock impedance (the product of density and shock velocity). These multiple shocks may be characterized by the methods described above. However, the flexible pulse technology proposed for the NIF will enable a more direct method for characterizing multiple shocks. In this case, the driving pulse (for either direct or indirect drive) is stepped in intensity. With appropriate target dimensions, we can arrange 
for the sample to be shocked in succession by these pulses.

\section{Isentropic Release States}

Isentropic release states are characterized by higher temperatures and lower densities than on the principal Hugoniot. These states can be reached by strong shocks followed by a release to lower pressure. When a shock in a material encounters a boundary with a medium of lower shock impedance, the final state of the material is on an isentropic release from the initial state. At a given pressure, these states will have higher temperatures and be more highly ionized than on the Hugoniot. If we think of the total pressure as arising from a "cold" compression part (that is, the $0^{\circ} \mathrm{K}$ isentrope) and from thermal contributions, these states are dominated by the thermal contributions to the equation of state.

When a strong shock in a material releases into a vacuum, it forms a rarefaction fan-a gradient of density and temperature. This is difficult to characterize. It is simpler to mount a material of very low shock impedance against the initially shocked material, and then measure the shock in the low-impedance material. The final state in that material will match, in mass velocity and pressure, the release state of the initially shocked material, so the release state can be characterized by simple Hugoniot measurements in the low-impedance material. ${ }^{19}$ The large target scales possible on the NIF will make this kind of experiment possible for the first time in a laserdriven environment.

This process is shown in schematic form in Figs. VI-18 and VI-19, which model the same shock experiment. Figure VI-18 models the experiment in terms of pressure history (vs time), and Fig. VI-19 shows the shock in terms of pressure vs volume.

Figure VI-18 shows the pressure history of the particle in the weakly shocked solid. The model is idealized and assumes that the solid behaves in a perfect-elastic, perfect-plastic way. The first shock wave (the elastic precursor) shocks the particle from normal pressure, density, and temperature to state $B$ at the Hugoniot elastic limit (HEL). The particle sits at this state $\left(B, B^{\prime}\right)$ until a second, slower wave (the plastic wave) shocks the solid to state $C$.

Peak pressure $C, C^{\prime}$ is maintained until the release wave, or rarefaction, reaches the particle at the release elastic limit (REL). The release wave is led by an elastic rarefaction (with a speed of $u_{3}$ ), which releases the solid to state $D, D^{\prime}$. This release is immediately followed by a slowermoving plastic rarefaction to the final state $\mathrm{E}$.

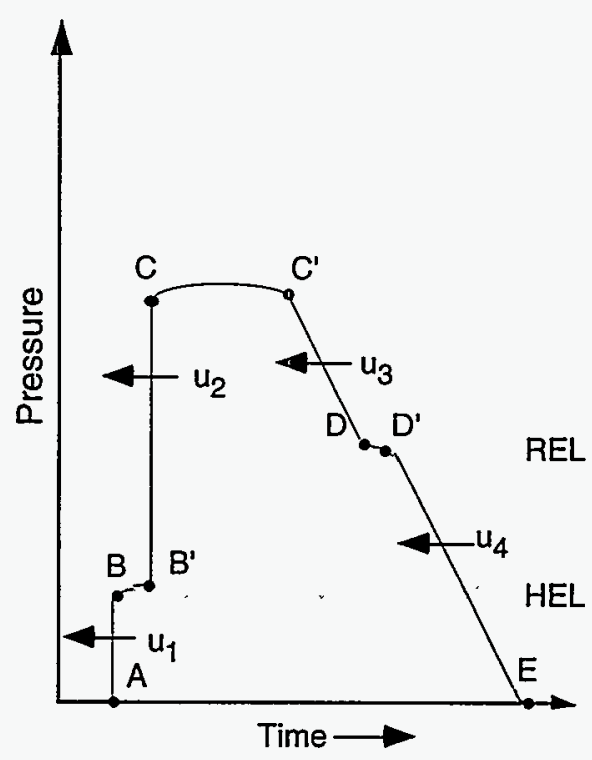

Figure VI-18. Model of pressure history vs time of a particle in a weakly shocked solid. In the initial state (A) the particle is at rest and at normal pressure, density, and temperature. The particle is taken from state $A$ to state $B$ discontinuously at the Hugoniot elastic limit (HEL), shown by the wide gray line at $B, B^{\prime}$, by the elastic precursor traveling at speed $u_{1}$. A second, slower wave (the plastic wave) shocks the solid from state $B, B^{\prime}$ to state $C$. Peak pressure $C, C^{\prime}$ is maintained until a release wave (or rarefaction) reaches the particle. The release wave, led by an elastic rarefaction, releases the solid to state $\mathrm{D}, \mathrm{D}^{\prime}$, at the release elastic limit (REL), shown by the narrower gray line. This release is immediately followed by a slowermoving plastic rarefaction to the final state $\mathrm{E}$. Elastic and plastic rarefactions travel faster than the plastic shock $\left(u_{3}>u_{2}\right)$, because the material these waves travel into is compressed and hot. With time, the release overtakes the shock, eroding peak compression. It is vital to ensure 
that the duration of peak compression $C, C^{\prime}$ is long enough to prevent decay of compression during the experiment, and this is where the NIF plays a significant role.

Figure VI-19 shows the Hugoniot, the locus of end points of shock compression, and the release isentropes for the above shock experiment.

At compressions up to the HEL, the solid responds to shock loading elastically-the lattice compresses in the same direction as the loading stress. The crystal lattice can support a shear stress, and the maximum shear stress the lattice can support defines the HEL.

When compressed beyond the HEL, material behavior changes significantly. At compressions above the HEL no further change in shear occurs (in the perfect-elastic, perfect-plastic model), and the material is said to yield. From the point of view of the material, a solid compressed beyond the HEL behaves in a fluid-like manner and starts to flow. This dramatic change in material response is observed in the principal Hugoniot as a cusp $\left(B, B^{\prime}\right)$. This change in material behavior in the weak shock regime is akin to a phase transition; hence the transition from state $B$ to state $B^{\prime}$. (No change in material properties is observed in the strong regime because only one shock, the plastic wave, exists. (See Fig. VI-20 for a model of the three shock regimes in solids.)

On a microscopic scale (which is still not fully understood), after state $C, C^{\prime}$ there is relaxation in the crystal lattice through dislo-cation movement and nucleation. The solid releases from state $C^{\prime}$ in two stages, first with an elastic rarefaction that moves it from peak compression state $C$ to some intermediate state $\mathrm{D}$ at the release elastic limit, along an isentrope. Next, a second, plastic, rarefaction returns the solid to the starting pressure, following a different isentrope to the end state E. Because shock compression is an irreversible process, the final state $E$ is different from the initial state $A$.

Figure VI-20 shows the three shock regimes in solids-the elastic regime, the weak shock regime, and the strong shock regime. In the elastic regime, the solid is shocked to less than the Hugoniot elastic limit (state B). This shock wave consists of a single elastic front. In the weak shock regime, the shock moves the

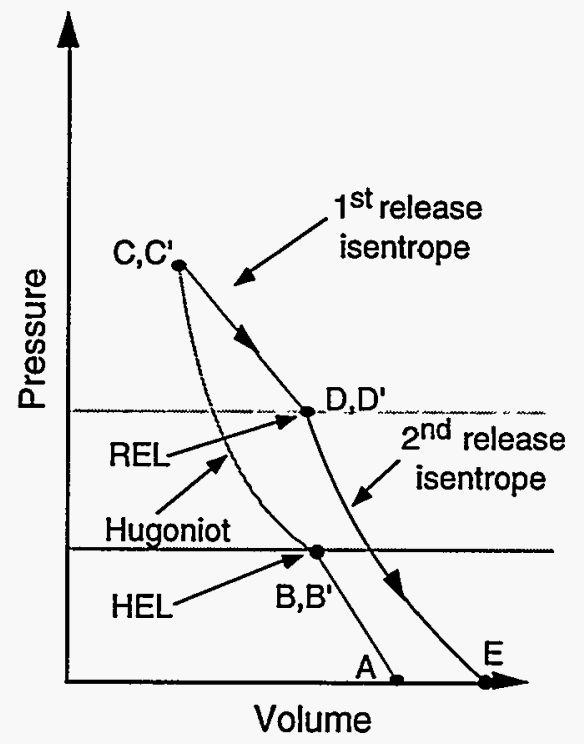

Figure VI-19. Model of pressure history vs volume, showing Hugoniot, end points of shock compression, and release isentropesfor the shock experiment above. As the elastic precursor takes the particle from state $A$ to state $B, B^{\prime}$ at the Hugoniot elastic limit (HEL), there is a dramatic change in material response, when no further change in shear occurs and the material is said to "yield." This is observed in the principal Hugoniot as a cusp $\left(B, B^{\prime}\right)$. On a microscopic scale, after state $C, C^{\prime}$ there is relaxation in the crystal lattice, and the solid releases from state $C^{\prime}$, first with an elastic rarefaction that moves the solid from peak compression (state $\mathrm{C}$ ) to some intermediate state $\mathrm{D}$ at the release elastic limit (REL), along an isentrope. Next, a second, plastic, rarefaction returns the solid to the starting pressure, following a different isentrope to the end state $E$. The final state $E$ is different from the initial state $A$ because shock compression is an irreversible process. 


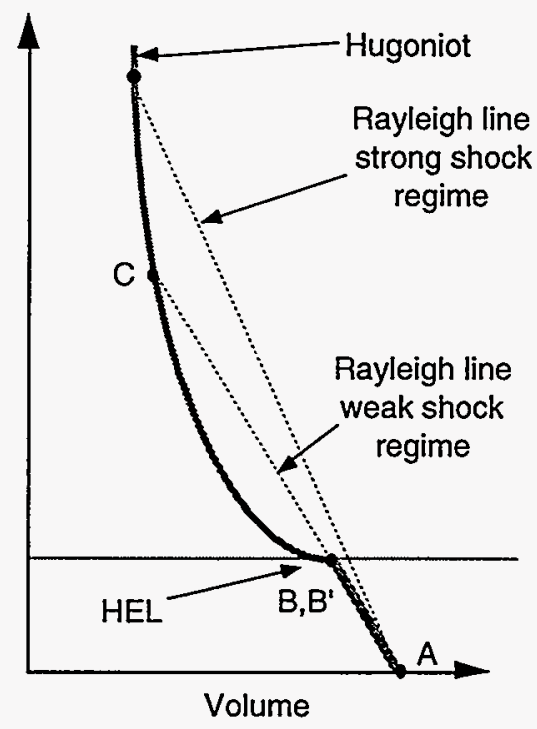

Figure VI-20. The three shock regimes in solids-elastic, weak shock, and strong shock regimes. Shock compression for a weak shock and a strong shock is drawn on a Hugoniot and the trajectories are shown as Rayleigh lines. Shock velocity is proportional to the slope; stronger shocks have higher shock velocities. In the elastic shock regime, the solid is shocked to less than the Hugoniot elastic limit (HEL) (state B). This wave consists of a single elastic front. In the weak shock regime, the shock moves the material continuously to the HEL (point B), at which point the material discontinuously goes to the intercept (point $\mathrm{C}$ ). The strong shock regime is defined by Rayleigh lines that have slopes equal to and greater than the straight line joining the HEL and the initial state. Here the double shock structure never develops, and a single discontinuous front is formed, taking the solid from state $A$ to a state of pressure higher than for the weak shock.

material continuously to the Hugoniot elastic limit (point B), at which point the material discontinuously goes to the intercept (point C). The final regime is the strong shock regime. Here the plastic waves acquire a velocity greater than the elastic precursor $\left(u_{2}>u_{1}\right)$, the double shock structure never develops. A single discontinuous front is formed, taking the solid from state $\mathrm{A}$ to a state of pressure higher than for the weak shock.

The conditions in a release experiment are found in nature both in high-energy-density ablation phenomena and in ultrahigh-velocity meteorite impacts. Thus, it is clear that understanding these states is important to understanding the shock generation process at the NIF itself. Since much of the radiation transport in laser-generated plasmas is in hot, expanded material, these states may provide a fruitful way to generate useful states for radiation transport studies.

\section{Isochoric Heating Experiments}

The use of isochoric heating (heating at constant density) on the NIF will enable an entirely new class of experiments at high pressure and temperature. This implies that the heating pulse will be absorbed in a time that is short compared to hydrodynamic time scales of expansion. It also implies a nearly constant deposition of energy throughout the sample volume. Isochoric heating will allow us to reach unique high-temperature and high-pressure states, and the high density will lead to strongly coupled physics. Note that other aspects of strongly coupled plasma are discussed in Section IX, Radiative Properties.

The unique states attainable by isochoric heating are in the center of a region for which no limiting equation of state theories apply and for which there is simply no data at all! These states fall outside the limits of low $\rho$, high $T$ where the activity expansions are valid, and outside the limits of high $\mathrm{T}$ where the Thomas-Fermi model is valid.

A promising method is to exploit the neutrons generated in a very short ( $<10-p s)$ pulse at ignition, with a total predicted energy of up to $45 \mathrm{MJ}$, mostly in $14-\mathrm{MeV}$ neutrons. We should be able to reach temperatures in thin foils of up to $50 \mathrm{eV}$ at solid density. Even in materials with small neutron cross sections, it will be possible to add small amounts of fissile material to increase the interaction with the neutrons without significantly changing the material equation of state. A free-standing foil heated in this manner will be an excellent test material for spectroscopy.

Another possible method to obtain isochoric heating is to use a set of the NIF beams to irradiate metal targets at high intensity in a short 
pulse. This will produce a copious amount of hard $\mathrm{x}$-rays. We calculate that a $10-\mu \mathrm{m}$-thick aluminum foil could be heated to tens of electron volts in this manner. In this case, it could be mounted to a low-Z stepped tamping foil; measuring the shock velocity in the tamper will allow determination of points on a unique release isentrope from the initial state. Again, such a well-characterized sample will be ideal for spectroscopy of high temperature and density states.

\section{$X$-ray Diffraction Studies}

The NIF will be able to push time-resolved in-situ $x$-ray diffraction into new experimental territory. Its higher energies and longer pulse lengths will allow creation of harder, brighter $x$-ray sources with longer durations. These $x$-ray sources will make available greater probe depths and experimental windows over which the shock compression process is measured. Thus, extensions of present techniques can then be used to study higher- $Z$ materials.

For example, it is not possible at present to experimentally investigate medium- $Z$ metals like iron and copper, where an extensive singlecrystal literature exists. Iron is especially important for geological reasons, and although it has been thoroughly investigated, there is still controversy over the phase diagram. ${ }^{20}$

The study of shock-induced polymorphic phase transitions, such as those observed in potassium chloride, is an area where longduration $x$-ray sources (i.e., 10-50 ns) that are bright enough to be time-resolved are of importance. ${ }^{21}$ Of further technological importance is understanding the effects of shock compression on superconducting properties, shock synthesis, and decomposition of high-temperature superconductors ${ }^{22}$ and ceramics. 23

All of these studies will require the extensive development of polycrystalline $x$-ray diffraction techniques. ${ }^{24}$ For $x$-ray diffraction techniques to work successfully on polycrystal-line material, the probing radiation needs to be extremely bright. This is because diffraction from a randomly oriented powder or poly-crystalline solid is much less efficient than from a single crystal. The lower diffraction efficiency is caused by the random orientation of the constituent microcrystals, which results in most of the material not presenting the Bragg angle to the incoming $x$-ray source. For polycrystalline material, a reduction of two orders of magnitude in diffracted intensity is expected when compared to the same material in the single crystal form.

In conventional $x$-ray diffraction analysis this reduced diffraction efficiency can be overcome by using long time integration to make a measurement, but even so, focusing cameras are necessary in some cases. In shock-wave physics, on the other hand, measurements must be taken on nanosecond time scales, requiring ultrabright $x$-ray sources and the use of $x$-ray focusing cameras.

In addition, the probing radiation needs to have a large penetration depth in order to sample a large volume. The standard method is to use a Seeman-Bohlin powder diffraction spectrometer, which allows the use of a large polycrystalline sample. Because of the long penetration depths of the hard $x$-rays that allow large volumes to be probed, powerful laser systems with NIF capabilities will be needed to create the ultrabright $x$-ray source at the short wavelengths preferred.

Figure VI-21 illustrates a Seeman-Bohlin camera (powder diffraction spectrometer). With this kind of camera, as shown schematically in the figure, $x$-rays from a divergent $x$-ray source are diffracted from a large-area powder or polycrystalline target. These diffracted $x$-rays are focused to a single line on the detector, helping to ease the intensity demands on the $x$-ray source. This increases the diffraction signal from a powder or polycrystalline sample.

Because a large-area sample is required, large-area shocks must be generated to compress the target. These shocks need to be uniform, which will require extremely large energies to create them. The need for high-flux, high-energy $x$-ray sources and large areas of shocked material indicate that experiments of this type will require the capability of the NIF. 


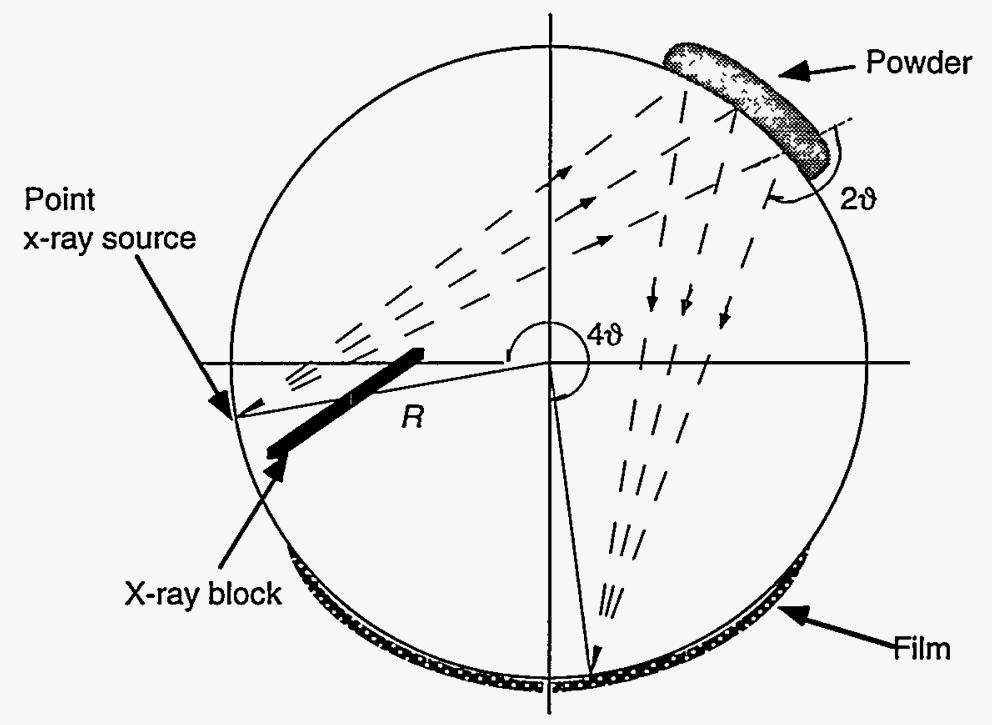

Figure VI-21. The Seeman-Bohlin camera. Diffraction from a randomly oriented powder or polycrystalline solid is much less efficient than from a single crystal. The Seemann-Bohlin camera diffracts $x$-rays, which come from a divergent source, from a large-area powder (or polycrystalline target). These diffracted $x$-rays are focused to a single line on the detector (the film), helping to ease the intensity demands on the $x$-ray source. In a large area sample an enormous number of randomly oriented microcrystallites are illuminated by the source, and those that are correctly aligned to the incident $x$-ray beam diffract onto the detector. The detector integrates (and increases) the signal.

In high-temperature superconductors, $x$-ray absorption is high because of the high $Z$ content (e.g., YBCO, BSCCO), which makes them difficult to study. Detailed quantum mechanical modeling of brittle fracture in ceramic titanium carbide exists, and shock breakout measurements have been shown to be one of the most successful ways of attaining and measuring ultimate tensile strains. ${ }^{25}$ This technique could be extended to study novel polycrystalline high-strength materials of the future.

The greater laser energies of the NIF can be used to generate larger compressions, making greater portions of the solid-state Hugoniot accessible to the $x$-ray diffraction technique. Because the compressions are large (up to $50 \%$ for a few megabars for many metals) the shocked area must subtend a large angle to the $x$-ray source. These shocked regions will need to be $2 \mathrm{~cm}$ in diameter or more. Such large-area shocks must be uniform to ensure uniaxial compression of the solid. Although uniform compression is not possible by direct radiation, it is possible with $\mathrm{x}$-ray driven ablation. ${ }^{26}$
Using long compression pulses is important to prevent the rarefaction from overrunning the shock front and eroding the compression. With $x$-ray-driven shocks, experimental investigation of shock front thicknesses in the strong shock regime can be made. Knowledge of the shock front, the thickness and shape, is extremely limited, and beyond the temporal and spatial resolutions of most experimental techniques.

With the next-generation facility's rapid rise time, long-duration compression waves should be possible using long-duration shaped laser pulses. Using such well-defined shocks allows us to use $x$-rays to probe only the material in the shock front that has strains between zero and peak compression. Diffraction at these intermediate angles must be related to the shock front. Using $x$-ray streak cameras with 1-ps time resolution, measurements with resolutions of approximately 30 atomic spacings are feasible. At these resolutions, issues such as shock planarity and target fabrication may dominate. These critical measurements of the shock front are vital for a fundamental under-standing of shock compression. 


\section{Other Material Properties}

While the emphasis above has been on basic high-pressure properties, other properties are very important scientifically. The electrical conductivity of materials at extreme conditions is crucial to understanding the origin of planetary magnetic fields, the transient fields predicted in the expansion plume of meteorite impacts, and MHD problems in general in hot and dense matter. Thermal conductivities are essentially unknown at pressures above $\approx 10 \mathrm{GPa}$. (Note: we have referred to radiation transport here and in other sections of this report-its importance is described in more detail in Section $\mathrm{X}$, Radiative Properties, Subsection E.)

All materials will be in hot fluid states at extreme shock pressures. For the group IV elements in particular, we note that these liquids are believed to be semiconductors or metallic. Their local structure is uncharacterized, but it is believed that for modest pressures above melt, the structure is not a close-packed liquid. This points out the need for techniques to determine the distribution function in space of ions or atoms in the post-shock or other transient highpressure states.

Another broad area of inquiry is the problem of non-equilibrium properties. This can be viewed as a special case of departure from local thermodynamic equilibrium (LTE), as applied to excitations of the system in general. We assume that areas of interest to material properties, such as those above, will rapidly evolve with the advent of the NIF. Until that time these are systems that cannot be considered ripe for investigation.

\section{E. References}

1. R. Cauble et al., ICF Quarterly Report 3,131 (1993).

2. R. Cauble et al., Phys. Rev. Lett. 70, 2102 (1993).

3. B. A. Hammel et al., Phys. Fluids B 5, 2259 (1993).

4. T. S. Perry et al., Phys. Rev. Lett. 67, 3784 (1991).
5. L. Da Silva et al., Phys. Rev. Lett. 69, 438

(1992); and D. Guenther, Nature 359, 585 (1992).

6. P.Springer et al., Phys. Rev. Lett. 69, 3735 (1992).

7. J. Wark et al., Phys. Rev. B 35, 9391 (1987).

8. R. R. Whitlock et al., in Shock Compression of Condensed Matter (North Holland, Amsterdam, 1991).

9. J. S. Wark et al., J. Appl. Phys. 68, 4531 (1990) and Phys. Rev. B 40, 5705 (1989).

10. See, for example, Stanley P. Marsh, LASL Shock Hugoniot Data (Univ. of Calif. Press, Berkeley, 1980); High-Velocity Impact Phenomena, R. Kinșlow, Ed. (Academic, New York, 1970); Ya. B. Zel'dovich and Yu. P. Raizer, Physics of Shock Waves and HighTemperature Hydrodynamic Phenomena, (Academic, New York, 1966).

11. E. Avrorin et al., JETP Lett. 43, 308 (1986); Sov. Phys. JETP 66, 348 (1987).

12. R. Cauble et al., Phys. Rev. Lett. 70, 2102 (1993).

13. Ya. B. Zel'dovich and Yu. P. Raizer, Physics of Shock Waves and High-Temperature Hydrodynamic Phenomena (Academic, New York, 1966).

14. A. C. Mitchell, W. J. Nellis, J. A. Moriarty, R. A. Heinle, N. C. Holmes, R. E. Tipton, and G. W. Repp, J. Appl. Phys. 69, 2981 (1991).

15. N. C. Holmes et al., in Proceedings of 8 th AIRAPT and 19th EHPRL Conference, C. M. Backman, T. Johannisson, and L. Thenér, Eds. (Uppsala, 1981).

16. W. J. Nellis et al., J. Chem. Phys. 79, 1480 (1983).

17. D. Saumon and G. Chabrier, Phys. Rev. $A 44$, 5122 (1991); Phys. Reo. A 46, 2084 (1992).

18. E. Avrorin et al., J. Sov. Phys. JETP 66, 348 (1987).

19. N. C. Holmes and J. A. Moriarty, Research Monthly, Lawrence Livermore National Laboratory, Livermore, California (to be published).

20. C. S. Yoo, N. C. Holmes, M. Ross, D. J. Webb, and C. Pike, Phys. Rev. Lett. 70, 3931 (1993). 
21. D. B. Hayes, J. Appl. Phys. 45, 208 (1974); E. B. Zaratskii, G. I. Kanel, P. A. Mogilevskii, and V. E. Fortov, Sov. Phys. Dokl. 36, 76 (1991).

22. Y. Syono and M. Kikuchi, in Shock Waves in Material Science, A. B. Sawaoka, Ed. (Tokyo, Springer-Verlag, 1993), p. 101.

23. T. Mashimo, in Shock Waves in Material Science, A. B. Sawaoka, Ed. (Tokyo, SpringerVerlag, 1993), p. 113.

24. N. C. Woolsey, Time resolved, in situ, $x$-ray diffraction from laser shocked solids, University of Oxford (1994); N. C. Woolsey, J. S. Wark, and D. Riley, J. Appl. Crystallogr. 23, 441 (1990).

25. D. L. Price, B. R. Cooper, and J. M. Wills, Phys. Rev. B 46, 11359 (1992).

26. T. Löwer, R. Sigel, K. Eidmann, B. I. Földes, S. Hüller, J. Massen, G. D. Tsakiris, S. Witkowski, W. Preuss, H. Nishimura, H. Shiraga, Y. Kato, S. Nakai, and T. Endo, Phys. Rev. Lett. 72, 3186 (1994). 


\section{Section VII}

\section{Plasma Physics}

The creation of and interaction with plasmas by high-energy lasers has a long history. ${ }^{1}$ The irradiation of a surface with a high-energy laser produces a blow-off plasma that can be quite hot and that contains densities suitable for the excitation of many parametric processes. Hence, high-power lasers represent a convenient way of producing plasmas with which to perform laserplasma interaction experiments.

The areas of plasma physics that have been addressed on high-energy lasers arise from two independent avenues of research. First, there are studies of the phenomena that are created by the laser interacting with a plasma. In this area one would have the instabilities enhanced by laserplasma coupling, such as stimulated Brillouin scattering, stimulated Raman scattering, etc. In the second there are attempts to use the laser to emulate other phenomena occurring in nature. Here the study of interpenetrating plasmas and plasma flow in a magnetic field are examples to be presented.

\section{A. Interpenetrating Plasmas}

Plasma interpenetration occurs in beamplasma systems and in laser ablation experiments, and in the context of geophysics and astrophysics. Therefore, interpenetration of counter-propagating plasmas can be considered a problem of fundamental interest in plasma physics. Single-fluid computer models are fundamentally unsuited for the study of interpenetration of counter-propagating plasmas because such codes enforce stagnation, resulting in unphysical temperatures and shock formation.
Although methods for modeling the collisionless regime are well developed, the intermediate regime is more difficult to model.

Recently, new computational methods have been investigated and applied to the simulation of colliding plasmas formed by laser ablation of parallel discs or foils. One modeling technique is to follow multiple fluids that are coupled by means of self-consistent electromagnetic fields as well as through collisional interaction.

A different approach is to augment kinetic particle-in-cell calculations with algorithms for collisions. A typical result from this latter method is presented in Fig. VII-1, which shows a calculation of two laser-heated parallel plastic foils. The figure shows the ion density for each foil at 800 ps after initiation of the laser, clearly illustrating the interpenetration of the plasmas into one another. The particle phase space view (A) shows the slowing down and heating in the center as the two plasma flows gently stagnate. The particle space result (B) shows that the interpenetration is stopped by collisions.

Figure VII-2 shows the schematic for an experiment performed on two solid targets. The two opposing laser beams impinge on the targets (one of aluminum and one of silicon) and create plasmas that expand toward each other. (The targets are of aluminum and silicon to permit spectroscopic identification of the plasma positions.) Measurements are made with a fourframe gated microchannel plate coupled to a filtered pinhole, so the images represent a twodimensional map of the emission. Figure VII-3 shows an $x$-ray image of the experiment shown schematically in Fig. VII-2. 
A) Phase space view

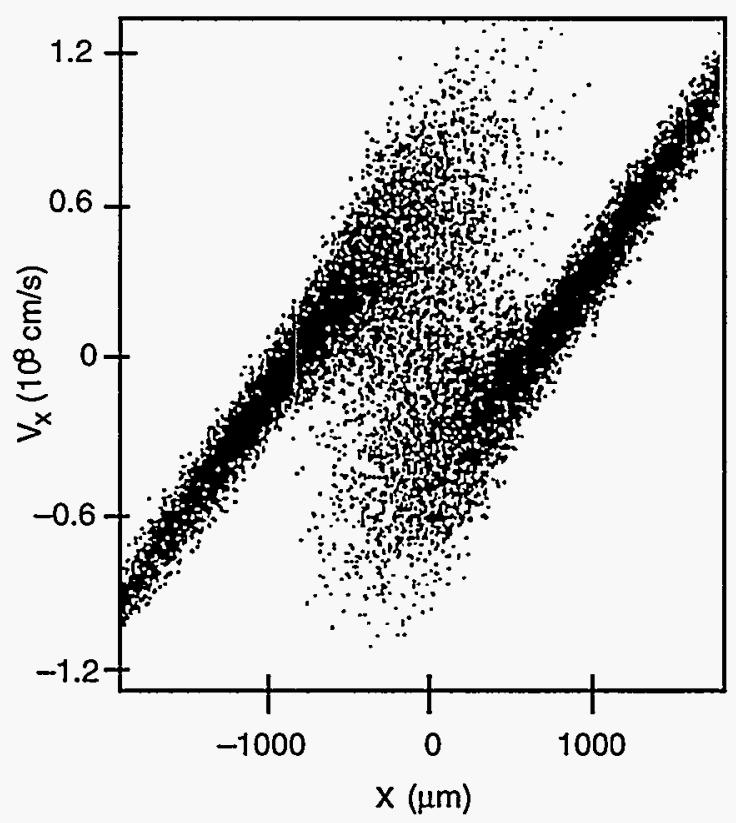

B) Particle space result

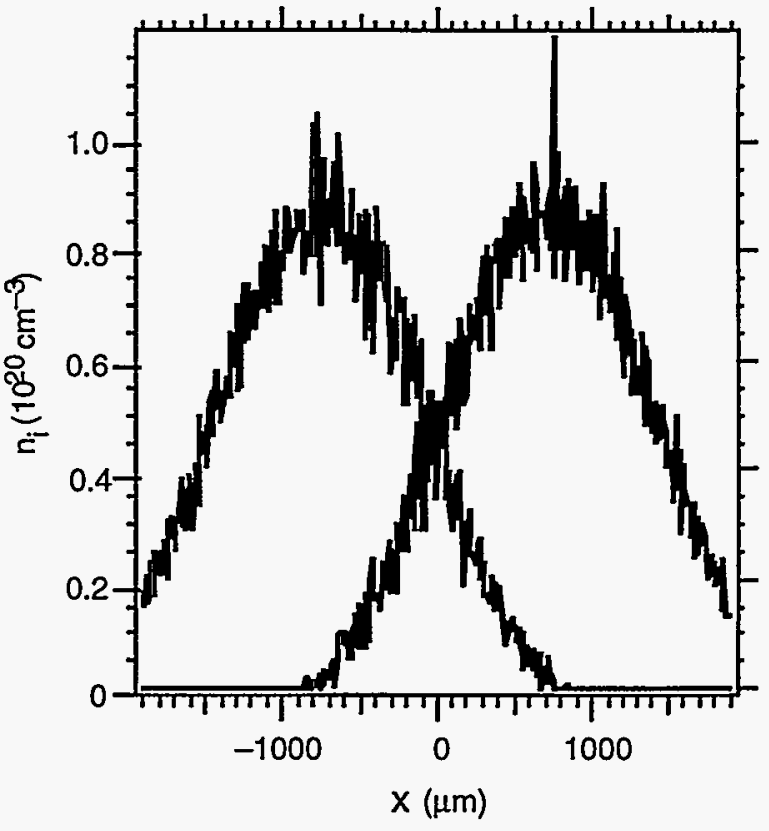

Figure VII-1. Simulation of an interpenetrating plasma showing two exploding carbon foils, originally $3.2 \mu \mathrm{m}$ thick, separated by $1500 \mu \mathrm{m}$. A) The phase space view, showing the stopping of the interpenetration by collisions. B) The particle space result, showing ion density vs position, measured from the center. The overlapping of the two curves clearly shows interpenetration. Both views are at 800 ps after initiation.

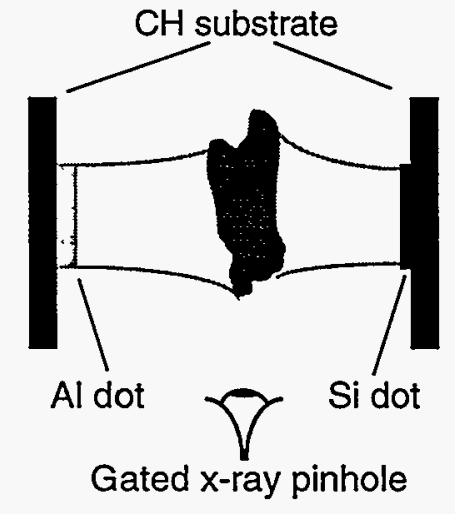

Figure VII-2. Schematic of colliding plasma experiment using two opposing beams to irradiate two facing solid targets. One target is aluminum (on the left) and one is silicon (on the right). The dots have a radius of $250 \mu \mathrm{m}$, the laser spot has a radius of $500 \mu \mathrm{m}$, and the distance between the dots is $800 \mu \mathrm{m}$.

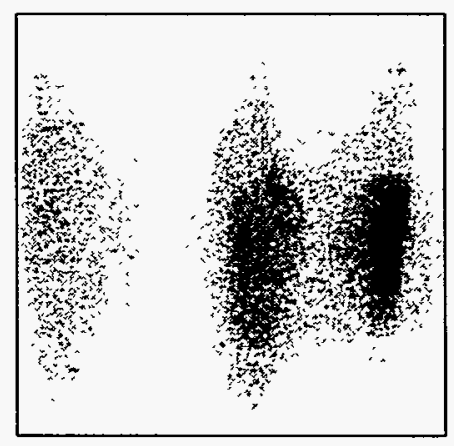

Figure VII-3. X-ray image of colliding plasma experiment shown in Fig. VII-2. The image was taken with a four-frame gated microchannel plate coupled to a filtered pinhole. 


\section{B. Plasma Streaming in Magnetic Fields}

The propagation and stability of collimated streams or jets of plasmas in magnetic fields is central to a number of important physical problems, including beam heating of magnetically confined thermonuclear plasmas, ${ }^{2}$ the interaction of the solar wind with planetary magnetospheres, ${ }^{3}$ and the formation and equilibrium properties of extragalactic jets. ${ }^{4}$

High-energy laser experiments conducted at total beam energies below $0.3 \mathrm{~kJ}$ have demonstrated the feasibility of studying highenergy jet phenomena with laser-produced plasmas..$^{5}$ A cylindrical glass hohlraum, $3000 \mu \mathrm{m}$ long by $1000 \mu \mathrm{m}$ diameter, was irradiated with one or two beams of a laser operating at $1.06 \mu \mathrm{m}$ wavelength. The laser had a pulse length of $2 \mathrm{~ns}$ and energies ranging from 0.03 to $0.3 \mathrm{~kJ}$. The hohlraum was located at the center of a $24-\mathrm{cm}$ diameter Helmholtz coil, which provided a spatially uniform and temporally constant magnetic field up to $10 \mathrm{kG}$ over regions and times of interest. The hohlraum was oriented with its axis perpendicular to the applied magnetic field.

The laser-produced plasmas from the inside of the cylindrical glass hohlraum were observed to evolve into plasma structures that were strongly collimated in the direction transverse to the hohlraum axis and the magnetic field, but jet- like in the direction parallel to the hohlraum axis. These jets were observed to propagate across the magnetic field lines at their initial velocities via the mechanism of an E $\times B$ drift. Shear in the velocity field of the jet led to the evolution of a classic Kelvin-Helmholtz-like instability on the edges of the plasma.

Figure VII-4 shows an optical image taken of one such expansion of a laser-produced plasma streaming into a $10-\mathrm{kG}$ magnetic field. The plasma is emerging from a hohlraum irradiated with a single beam at $0.1 \mathrm{~kJ}$. The image was taken at $850 \mathrm{~ns}$ after the laser pulse started, and was obtained using an ultrafast optical framing camera with 2-ns time resolution. In this experiment, the hohlraum end opposite to the laser-entrance hole was closed using an epoxy plug.

At $850 \mathrm{~ns}$ after the laser was incident on the target, the maximum plasma jet velocity is $\approx 8 \mathrm{~cm} / \mu \mathrm{s}$ and a dramatic vortex structure has been produced. Both the jet formation and the observed instability may be relevant to a range of astrophysical phenomena.

\section{Laser-Plasma Instabilities}

The coupling of high-intensity laser light to plasmas has been the subject of experimental investigations for many years. ${ }^{6}$ These experiments have focused on measuring a broad

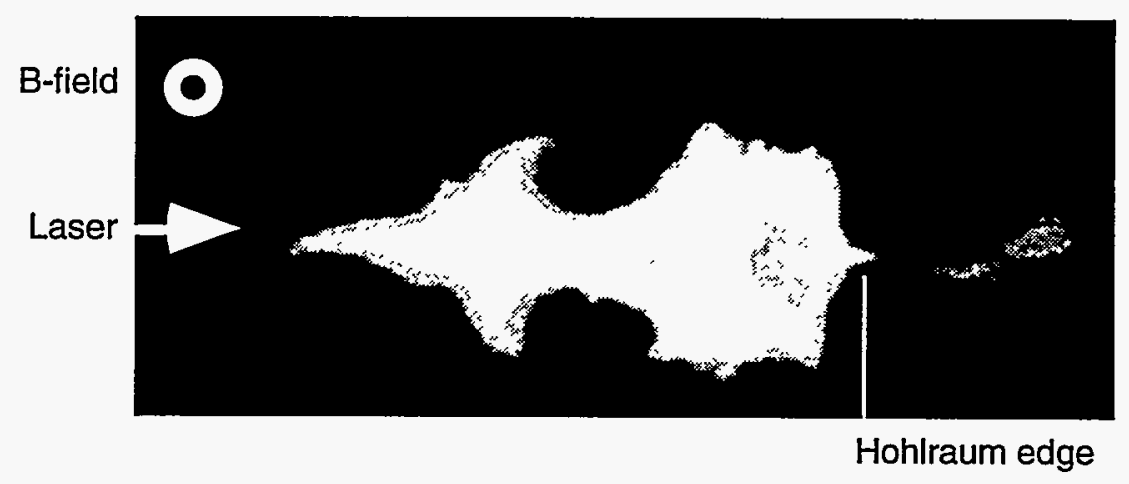

Figure VII-4. Optical image of a plasma streaming into a magnetic field. The plasma jet is emerging from a hohlraum on the right into a B-field that is coming out of the plane of the figure. The image was taken at $850 \mathrm{~ns}$ after the laser pulse started, using an ultrafast optical framing camera with 2-ns time resolution. Note the dramatic vortex structure. 
range of phenomena such as resonance and collisional absorption, filamentation, density profile and particle distribution modification, and the growth and saturation of various parametric instabilities. These phenomena depend on both the properties of the laser (intensity, wavelength, pulse length coherence, etc.) and the composition of the plasma.

Experimental studies of laser-plasma instabilities have become particularly important in recent years as a result of the vigorous research effort in laser-driven inertial confinement fusion (ICF). The success of ICF depends partly on mitigating the undesirable effects of two particular parametric instabilities, stimulated Raman scattering and stimulated Brillouin scattering.

These two instabilities are of particular importance because both degrade the target compression efficiency in a spherical implosion experiment. Electron Landau damping from the stimulated Raman scattering instability produces fast electrons that can preheat the core of an imploding sphere prior to the arrival of the compression shock front. ${ }^{7}$ The stimulated Brillouin scattering instability can scatter a substantial fraction of the incident laser light, causing an overall reduction in the laser-to$x$-ray drive efficiency and modifying the $x$-ray drive symmetry.

In addition to its importance for ICF, highintensity laser-plasma coupling presents an extraordinarily rich topic in the study of highenergy-density physics. For example, laserproduced plasmas provide a unique environment for the study of collisional and resonance absorption of laser light. Numerous experiments using various kinds of target materials and widely varying laser parameters have verified the general features of collisional absorption, such as the dependence of absorption on plasma temperature, scale length, laser wavelength and intensity. 8,9

Experiments investigating resonance absorption, which occurs at the critical density surface, show an expected dependence on the angle of incidence and polarization of the laser. ${ }^{10,11}$ However, these experiments also show some discrepancies in the absorbed energy that may be attributed to rippling of the critical density $\left(\mathrm{N}_{\mathrm{cr}}\right)$ surface. Density profile modification has been observed in experiments where resonance absorption is the dominant coupling mechanism. This profile modification can lead to harmonic generation in back-reflected laser light. ${ }^{12}$

Laser-produced plasmas provide a unique environment for the study of parametric instabilities. These instabilities can most simply be described as the resonant coupling of the incident laser light into two other plasma waves. Experiments have been done on stimulated Raman scattering instability (discussed above in the context of ICF), where an incident photon decays into an electron plasma wave and a scattered photon. These experiments have shown some important trends, such as the generation of hot electrons from stimulated Raman scattering and the dependence of the instability on plasma scale length, laser intensity, and electron collisionality.

Figure VII-5 shows the region in a density gradient where stimulated Raman scattering and Brillouin scattering play a role. The figure shows that occurrence of Brillouin scattering, filamentation, and inverse bremsstrahlung is possible at places below the critical density, while the wave-matching conditions indicate that two-plasmon decay and stimulated Raman scattering occur at and below the quarter critical density point.

The occurrence of the stimulated Raman scattering and the two-plasmon decay is separated into two density regimes. The first region is near quarter critical density, where the stimulated Raman scattering and two-plasmon decay can occur together, with both being absolute instabilities. This means these instabilities can grow, saturate, create ion waves, and create density increases. Then the waves cease to satisfy the wave-matching conditions. At this point the profiles relax, allowing the process to re-initiate. However, in the lower-density regime the stimulated Raman scattering is not so strongly damped and the process is convective. 


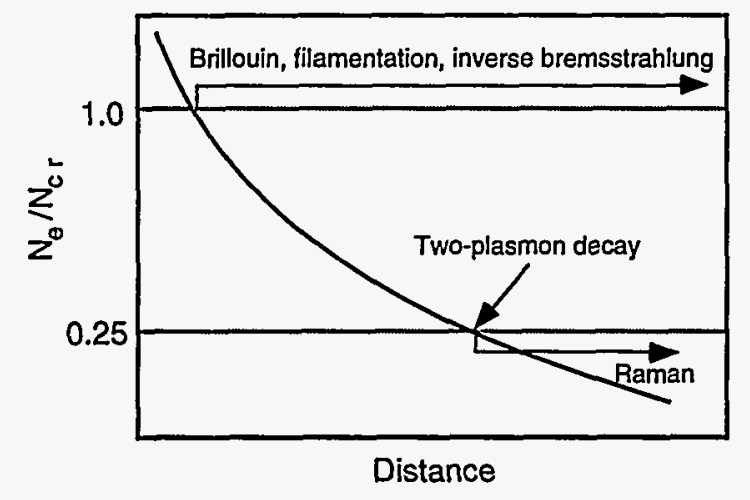

Figure VII-5. Schematic of regions where laserplasma instabilities can occur in an electrondensity gradient. Electron density (in units of the critical density) for an idealized gradient is shown as a function of distance. The occurrence of Brillouin scattering, filamentation, and inverse bremsstrahlung is possible in regions below the critical density, while the wavematching conditions indicate that two-plasmon decay and stimulated Raman scattering occur at and below the quarter critical density point. The occurrence of the stimulated Raman scattering and the two-plasmon decay is separated into two density regimes. The first is near quarter critical density, where the stimulated Raman scattering and two-plasmon decay can occur together, with both being absolute instabilities. In the lower-density regime the stimulated Raman scattering is not so strongly damped and the process is convective.

\section{Stimulated Raman Scattering}

The wave-matching conditions for stimulated Raman scattering are shown schematically in Fig. VII-6. Here the incident laser interacts with the plasma, stimulating a scattered light wave and an electron plasma wave.

Figure VII-7 shows an example of the timeresolved stimulated Raman scattering spectrum arising from a $\mathrm{CH}_{2}$ exploding-foil target. The foil was irradiated with a temporally square 1.0-ns pulse of $0.35 \mu \mathrm{m}$ light with an intensity of $\sim 3 \times 10^{15} \mathrm{~W} / \mathrm{cm}^{2}$. The laser burned through the target during the pulse, producing an approximately parabolic density profile. This is seen in the figure, where the maximum density at which the stimulated Raman scattering occurs

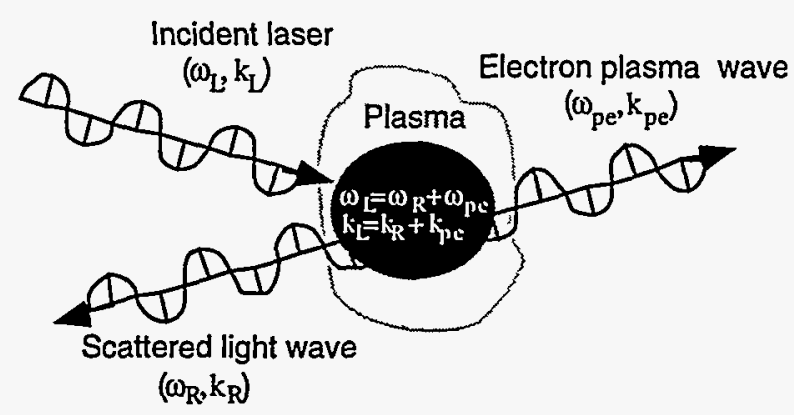

Figure VII-6. Wave-matching condition for stimulated Raman scattering. The incident laser interacts with the plasma, stimulating a scattered light wave and an electron plasma wave.

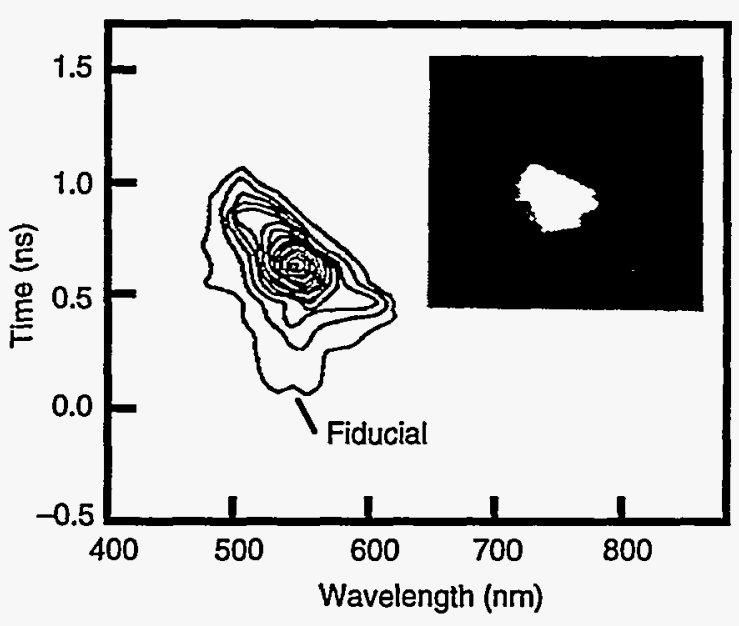

Figure VII-7. Contour plot of intensity of Raman-scattered light vs wavelength and time for a $\mathrm{CH}_{2}$ exploding-foil target. It can be inferred that the maximum density at which the stimulated Raman scattering occurs drops from $\sim 0.22$ to $0.09 \mathrm{~N}_{\mathrm{cr}}$ as the foil explodes. The insert shows the data image from a streak camera coupled to a visible spectrometer. The experiment has a thin plastic foil irradiated by a $0.35-\mu \mathrm{m}$ laser at $10^{15} \mathrm{~W} / \mathrm{cm}^{2}$. Note the timing fiducial used to indicate time relative to the incident laser pulse.

drops from $\sim 0.22$ to $0.09 \mathrm{~N}_{\mathrm{cr}}$ as the foil explodes. Further, the time dependence of the shortwavelength cutoff implies a heating of the electrons followed by cooling as the foil expands. However, the details of this instability (such as the frequency spectrum, angular distribution of 
the scattered light, saturation mechanisms, threshold intensities, and starting noise level for the instability) are not well understood.

Two-plasmon decay instability occurs when the incident laser light decays into two electronplasma waves. Experiments utilizing Thomson scattering have shown that the wave number for maximum growth is the same as that predicted by linear theory. ${ }^{13}$ These same experiments showed large levels of (short-wavelength) ion fluctuations that correlated with the twoplasmon-decay Langmuir waves. Meanwhile, experiments have examined the saturation and long-time-scale evolution of this instability. ${ }^{14}$

\section{Stimulated Brillouin Scattering}

The stimulated Brillouin scattering instability occurs when the incident laser decays into an ion acoustic wave and a scattered light wave. There have been a number of interaction experiments that have produced substantial backscattered and sidescattered light that is attributed to the stimulated Brillouin scattering instability. 8,15 The intensity of the scattered light seems to decrease as the wavelength of the interaction light decreases.

The temporal growth rate, spatial behavior, and saturation characteristics of stimulated Brillouin scattering have been investigated using Thomson scattering and measurements of the back-reflected light. ${ }^{16}$ The exponential growth in time of the scattering was found to be in good agreement with linear theory. Experiments investigating the nonlinear effects of stimulated Brillouin scattering have shown ion acoustic wave harmonic generation and a rapidly varying $100 \%$ modulation of the ion wave. ${ }^{17}$ The stimulated Brillouin scattering process has been studied in the past because of its creation of a large reflected wave, which diminishes the coupling of an intense laser.

Figure VII- 8 shows the wave-matching conditions for stimulated Brillouin scattering. The physical mechanism that generates the reflected wave is shown in Fig. VII-9. An impinging laser of electric field, $E_{L}$ interacts with initial density fluctuation, $\delta$, to produce a transverse current proportional to $\delta \mathrm{nE}_{\mathrm{L}}$. This transverse current produces a reflected light wave with a field $E_{R}$.

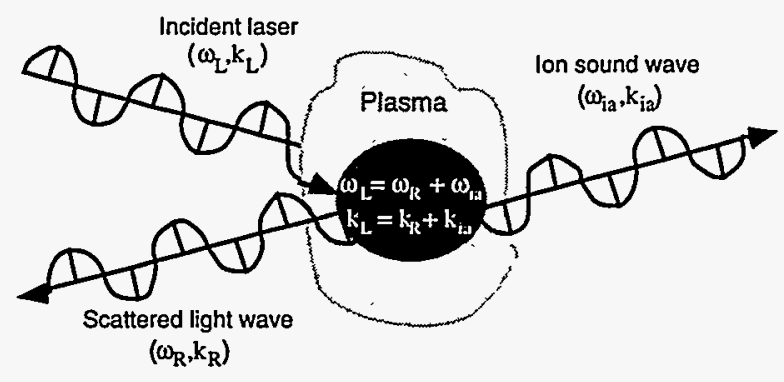

Figure VII-8. Wave-matching condition for stimulated Brillouin scattering. The stimulated Brillouin scattering process creates a large reflected wave, which diminishes the coupling of an intense laser.

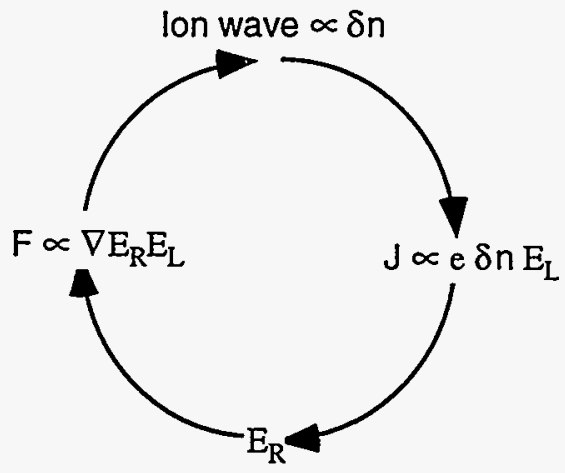

Figure VII-9. Diagram of physical mechanism that generates the reflected wave. An impinging laser of electric field, $E_{L}$, interacts with initial density fluctuation, $\delta n$, to produce a transverse current proportional to $\delta \mathrm{nE}_{\mathrm{L}}$. This transverse current produces a reflected light wave with a field $E_{R}$. Then the ponderomotive force due to the incident and reflected light waves, $\mathrm{E}_{\mathrm{L}} \mathrm{E}_{\mathrm{R}} / 8 \pi$, can in turn enhance the density fluctuation, $\delta n$. This enhancement will grow as long as the wave-matching conditions shown in Fig. VII-8 are met.

Then the ponderomotive force due to the incident and reflected light waves, $\mathrm{E}_{\mathrm{L}} \mathrm{E}_{\mathrm{R}} / 8 \pi$, can in turn enhance the density fluctuation, $\delta \mathrm{n}$. This enhancement will grow as long as the wavematching conditions are met.

Stimulated Brillouin and Raman scattering are three-wave processes that occur when the incoming light photon (of frequency $\omega_{0}$ and wave number $k_{0}$ ) couples to a scattered light photon 
and a plasma wave. The conservation equations that govern the interactions are:

$$
\begin{aligned}
& \omega_{0}=\omega_{s c a t}+\omega_{p w w} \text { (energy conservation) } \\
& k_{0}=k_{s c a t}+k_{p w} \text { (momentum conservation) }
\end{aligned}
$$

The subscripts scat and $p w$ refer to the scattered light photon and the plasma wave respectively.

Brillouin scattering with its reflected wave has many interesting features that will be addressable at the NIF. Among these are saturation mechanisms; ion heating; and instability competition between, for example, stimulated Raman and stimulated Brillouin scattering.

Some typical spectra of back-reflected light for a glass microsphere and a plastic disk irradiated with 1.06- $\mu \mathrm{m}$ light are shown in Fig. VII-10. Here the spectrum is red-shifted for the higher- $Z$ target shown, while the spectrum is blue-shifted for the low- $Z$ plastic target. The spectral shift has been measured with different angles of incidence in order to estimate sound speed and plasma expansion velocity. Note that experiments using spatial and temporal laserbeam smoothing techniques, mentioned in the description of the laser facility, have shown a significant reduction in the level of backscattered emission from both stimulated Brillouin and stimulated Raman scattering instabilities. ${ }^{18}$

More than one parametric instability may occur at the same location in a plasma, and these can interact with each other. One experiment investigating the interaction of stimulated Raman scattering instability and stimulated Brillouin scattering showed a strong correlation between the quenching of stimulated Raman scattering instability plasma waves and the initiation of stimulated Brillouin scattering ion waves. ${ }^{19}$

Another experiment showed that the spectrum of backscattered light from stimulated Raman scattering instability depended on the presence of stimulated Brillouin scattering. ${ }^{20}$ The data in these experiments suggest an explanation for the temporal evolution of the "Raman gap," an observationally missing stimulated Raman scattering instability signal that would be associated with regions of electron density from $\sim 0.18$ to $0.25 \mathrm{~N}_{\mathrm{cr}}$. The data showed that the "Raman gap" could be explained in terms of the stimulated Brillouin scattered light. Although
A) Higher-Z target

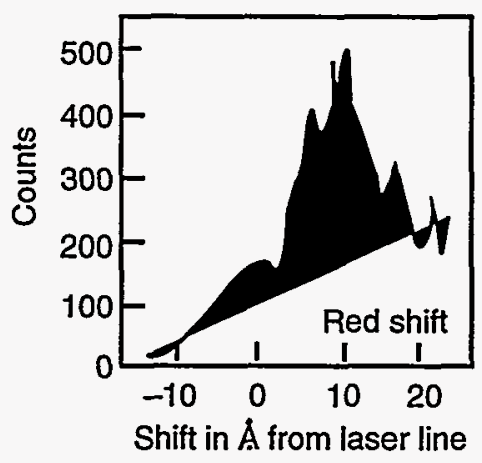

B) Low-Z plastic target

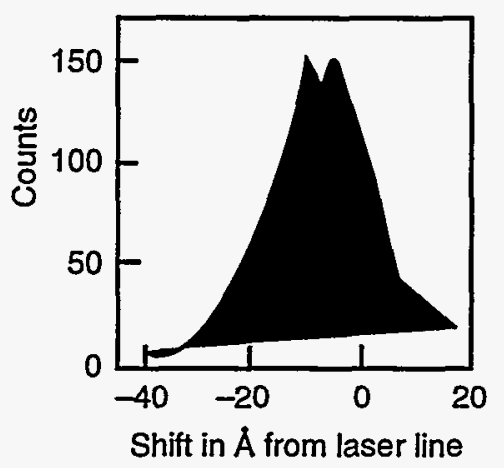

Figure VII-10. Spectra for the back-reflected light due to stimulated Brillouin scattering. In A) the material is a glass microsphere (the higher- $Z$ target), while in $B$ ) it is a parylene- $C$ disk (the low-Z plastic target). The spectrum for the higher- $Z$ target is red-shifted, while for the low- $Z$ target it is blue-shifted.

these experiments do not completely explain the gap, the results are consistent with the assumption that an interaction exists between the two instabilities.

\section{Filamentation Instability}

Filamentation instability occurs when a small hot spot in the laser-intensity profile undergoes self-focusing as a result of thermal, ponderomotive, and/or relativistic effects. The result is that electrons, and eventually ions, are expelled from the filament, lowering plasma density and causing the laser light to focus more tightly. This creates an unstable feedback loop where a tighter focus leads to higher intensity and more density depletion. Eventually, the instability saturates 
through diffraction effects, thermal absorption, or laser-light scattering by stimulated Brillouin and Raman scattering instabilities.

Filamentary structures have been inferred from density perturbations measured using interferometry, shadowgraphy, and dark-field imaging, and by imaging second and threehalves harmonic emission. ${ }^{21,22}$ Other experiments designed to study the growth of a well-defined intensity perturbation show an increase in the perturbation of spatial density that is consistent with ponderomotive filamentation. ${ }^{23}$

Figure VII-11 shows an example of laserinduced filamentation results for an experiment using a long scale-length plasma. The plasma was formed by focusing a 4-ns pulse of $1.05-\mu \mathrm{m}$ light onto a plastic foil. The pulse had a spot size of $\sim 1 \mathrm{~mm}$ and a peak intensity of $\sim 6 \times 10^{12} \mathrm{~W} / \mathrm{cm}^{2}$. The plasma was then irradiated with a second, more intense, 300-ps pulse of 1.05- $\mu \mathrm{m}$ light, this one with a peak intensity of $\sim 8 \times 10^{14} \mathrm{~W} / \mathrm{cm}^{2}$, creating a second-harmonic emission. The figure shows an image of the pattern of the secondharmonic emission.

\section{Future NIF Experiments}

In the past, laser-produced plasmas have not been sufficiently uniform, and so the interaction physics, which is extremely sensitive to gradients, has been clouded. Some of the plasmaphysics phenomena that will be studied with a large laser like the NIF are grouped here for discussion. In some cases, knowledge of the behavior of the phenomena is quite extensive and deeper understanding is sought. In other cases, the phenomena have not been addressed experimentally at all. This difference in treatment is due to the level of sophistication we now have in the various areas.

The experiments envisioned in the present discussion would require good focal-spot focusing capability - as small as $50 \mu \mathrm{m}$ FWHMand a dedicated interaction beam line with very high or low $\mathrm{f} /$ number as well as the potential to operate with laser wavelengths at first, second, and third harmonics. The experiments would also need some type of Thomson-scattering probe beam plus collection optics. These experiments

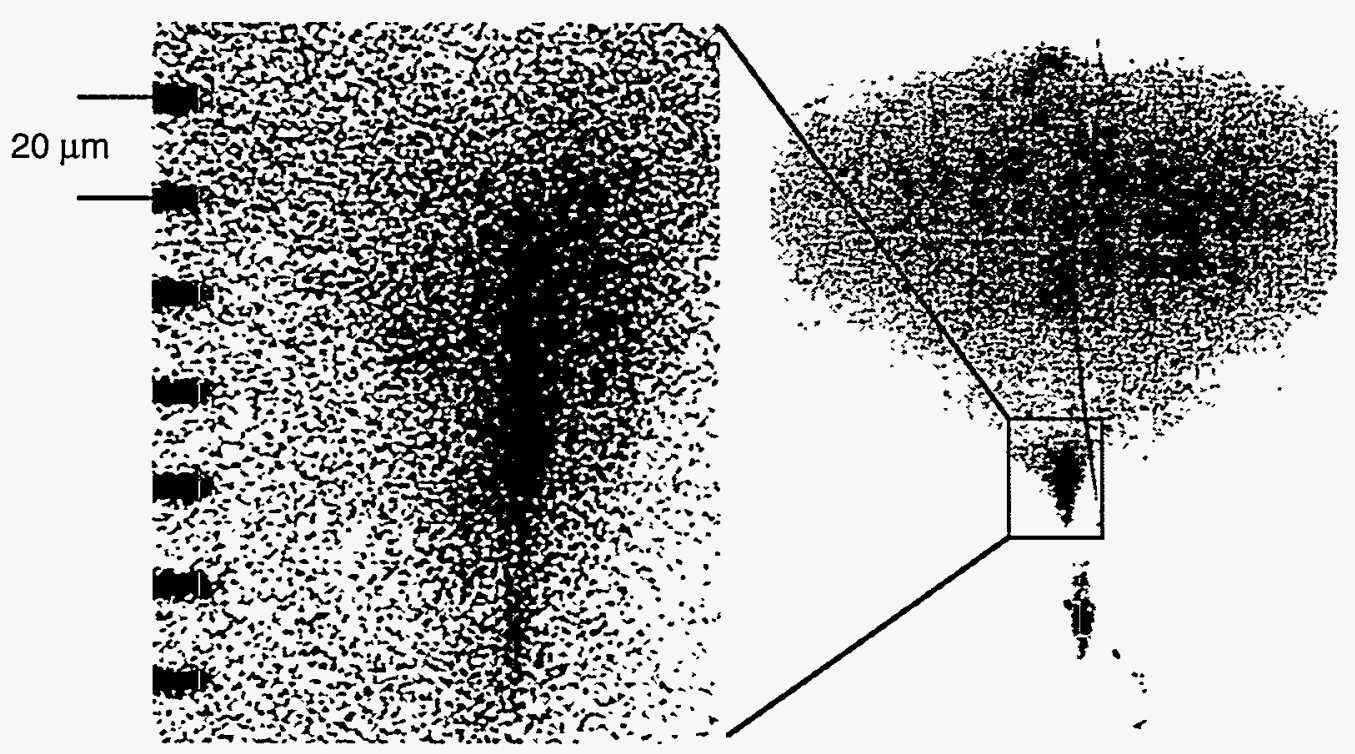

Figure VII-11. Image of laser-induced filamentation. The second-harmonic emission is created by irradiating a preformed plasma with a second, intense $1.05-\mu \mathrm{m}$ laser. The left image (with the scale) shows a magnified section of one of the filamentary structures. The image on the right shows the target surface at the top together with an entire spatial record. 
require the energy of the NIF to produce the large preformed plasma needed to obtain large interaction lengths.

The NIF's proposed 1.8-MJ laser will allow the production of a plasma at an electron temperature of $3 \mathrm{keV}$ with an $11-\mathrm{mm}$ diameter and a plasma at 5-6 keV with a 6-mm diameter. (With smaller plasmas, the NIF will be able to produce even higher electron temperatures.) The larger plasmas will translate into longer interaction lengths, greater homogeneity, higher temperatures, longer time scales, and reduced velocity gradients. Thus, the NIF is central to the experimental possibilities discussed below.

\section{Laser-Plasma Interactions in Large, Hot Plasmas}

\section{Long Scale-Length Plasma Production}

Several kinds of diagnostics can be used to study laser-plasma interactions in large, hot plasmas. One primary diagnostic for filamentation would be stimulated Brillouin scattering signal, which is indicative of lower density in the filaments that could be coupled with high spatial resolution (on the order of $1 \mu \mathrm{m})$. Other diagnostics would be XUV imaging and high-resolution optical probing with 4- $\omega_{0}$ light. Further, the study of the angular distribution of scattered light would provide an indication of filamentation.

Thomson scattering could be used for these investigations, thus making highly localized measurements of plasma temperature and density by scattering from thermal electrondensity fluctuations in the plasma. In addition to using Thomson scattering for thermal measurements, it is possible to reach far deeper understanding of the physics of laser-plasma instabilities by using Thomson scattering to measure the coherent motion of electrons involved in ion acoustic and electron plasma waves. This measurement can provide a temporally and spatially resolved measure of the coherent fluctuation amplitude in a specific direction determined by the angle made by the detector, scattering volume, and scattering light source.
Measurement of the background fluctuation levels would provide information about the initial level of coherent fluctuations, the amplification of these levels with the application of a pump or interaction beam, and the saturation level of the fluctuations. In addition, it could provide useful information about the coupling between stimulated Raman and stimulated Brillouin scattering by means of simultaneous measurement of the ion acoustic and electron plasma waves at the same spatial location.

Measurement of optical-wavelength Thomson scattering is well-suited for studying low electron densities such as are encountered in the corona, whereas $\mathrm{x}$-ray wavelength Thomson scattering is better-suited for studying plasma densities that exceed the critical density for standard visible and near-UV lasers. The possibility of developing an $x$-ray Thomson scattering measurement to study coherent plasma motion in high-density plasmas is an exciting one.

Present open-geometry plasmas, called "gasbags," have produced large (millimeterscale) plasmas at interesting electron temperatures. A schematic of the experiments that produced these large-scale plasmas is shown in Fig. VII-12, along with the diagnostic complement that is available. The gasbag is formed of two plastic films of thickness $0.6 \mu \mathrm{m}$ clamped by a hoop to form a bag. Pressure in the gasbag is stabilized using a pressure transducer at densities such that the fully ionized species yield electron densities of $\sim 10^{21} \mathrm{~cm}^{-3}$. There is a separate interaction beam (indicated by the light blue arrow) to drive the instabilities in a controlled way. For the NIF this geometry should be directly scalable to approximately $4 \mathrm{~cm}^{3}$, using $9 / 10$ of the NIF laser to form the plasma and $1 / 10$ to create interactions. The uses for this type of large uniform system range from plasma interaction to nuclear reaction rate studies.

Figure VII-13 shows the time history, in twodimensional x-ray images, of the emission of a gasbag. The sequence of images demonstrates the uniformity of the plasma in a gasbag. Images were taken at three times during the heating pulse, using a gated $x$-ray imager, and represent 
frame times of $100 \mathrm{ps}$ each. At initiation of the laser the plasma is a glowing emission of the outer shell of the plastic membrane bag. At $425 \mathrm{~ns}$, after the laser has heated the plasma, it is uniform. By 875 ps, the gas can be seen to be extremely uniform. This gasbag technique holds much promise with large laser systems like the NIF because scaling of this kind of experiment to large systems is straightforward.

There have been many plasma physics experiments that have been virtually impossible to interpret quantitatively. Coupling the capability to make large, uniform hot plasmas with the ability to control perturbations of the plasma (for example, by the use of higher density materials nearby or controlled flow velocities) will allow us to study the fundamental aspects of these experiments. As an example one can look at the plasma-physics experiments that focus on the hohlraum environment.

\section{Stimulated Brillouin and Raman Scattering}

As mentioned above, the study of laserplasma interactions and parametric instabilities such as stimulated Raman scattering (SRS) and stimulated Brillouin scattering (SBS) are of great interest to many researchers. ${ }^{24,25}$

Plasmas can support different types of waves. In stimulated Brillouin scattering, an "ion-acoustic" wave is created, which is the collective motion of the ions and electrons in a sound wave. These have substantial inertia.

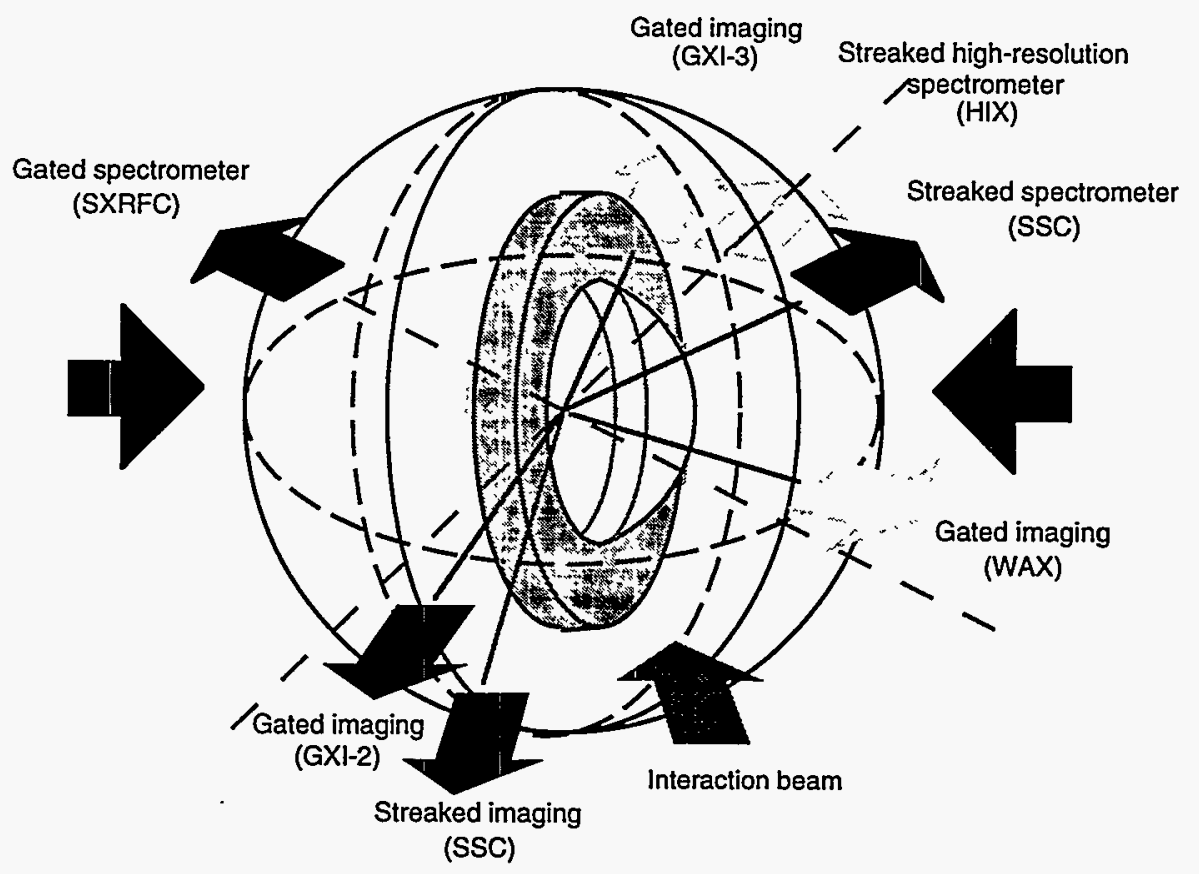

Figure VII-12. Schematic of the formation of a large-volume hot plasma. The figure shows both the gasbag used to create a large-scale plasma, and the diagnostic complement that is available. The gasbag (light gray sphere) is formed of two plastic films of thickness $0.6 \mu \mathrm{m}$. The hoop (shown in darker gray) holds the two plastic films clamped to form the bag. The gas bag is filled through two tubes in the hoop. Pressure in the gasbag is stabilized using a pressure transducer at densities such that the fully ionized species yield electron densities of $\sim 10^{21} \mathrm{~cm}^{-3}$. The roughly spherical volume of this plasma is $0.066 \mathrm{~cm}^{3}$, with a radius of $2.5 \mathrm{~mm}$. The gas in the volume is heated to temperatures that are on the order of $3000 \mathrm{eV}$. The diagnostic complement access is indicated by the light gray arrows, while the heating lasers are shown on either side in black. There is a separate interaction beam (indicated by the medium gray arrow pointing in) to drive the instabilities in a controlled way. For the NIF this geometry should be directly scalable to approximately $4 \mathrm{~cm}^{3}$, using $9 / 10$ of the NIF laser to form the plasma and $1 / 10$ to create interactions. 
In stimulated Raman scattering, an "electronplasma" wave is created, which is the collective oscillation of the plasma electrons at the plasma frequency, oscillating about the stationary ions. Beating between the incident and scattered light wave serves to enhance the plasma wave, producing more scattering of the incident light. The result is a feedback loop that, in times on the order of picoseconds, produces an exponential increase in scattered signal. This signal saturates at anywhere from 10 to $100 \%$ of the incident light.

Processes such as Brillouin and Raman scattering are obviously important from the point of view of transporting laser light effectively through a plasma, as is the case in inertial confinement fusion. The scattering of light at large angles is detrimental to the symmetry of ICF experiments because it results in energy being deposited in the wrong place. Another example is the effect of SRS on an electronplasma wave. The by-product of stimulated Raman scattering is the conversion of the electron-plasma wave energy into extremely fast electrons, from $50 \mathrm{keV}$ to $1 \mathrm{MeV}$ energies. These fast electrons not only can transport energy away from the experiment, but can also deposit it within a target, giving rise to undesirableheating.

Because laser-plasma instabilities are the subject of numerous studies, and instabilities form a challenging area of plasma physics, the NIF's large uniform plasmas and laser-pulse shaping coupled to the diagnostics possible on the NIF make it an ideal site for studying these processes.

Sidescatter is of major interest to plasma physicists. This is because in strong gradients with large laser spots, sidescatter is predicted to dominate backscatter. Further, the behavior of sidescatter will be different in flowing and stationary plasmas. Theoretical and experimental understanding of sidescatter is incomplete, so this is an area that will benefit greatly from experiments on the large uniform, controllable plasmas that the NIF will afford.

\section{Filamentation}

Filamentation instability occurs when a small hot spot in the laser intensity profile undergoes self-focusing as a result of thermal, ponderomotive, and/or relativistic effects. ${ }^{26}$ The
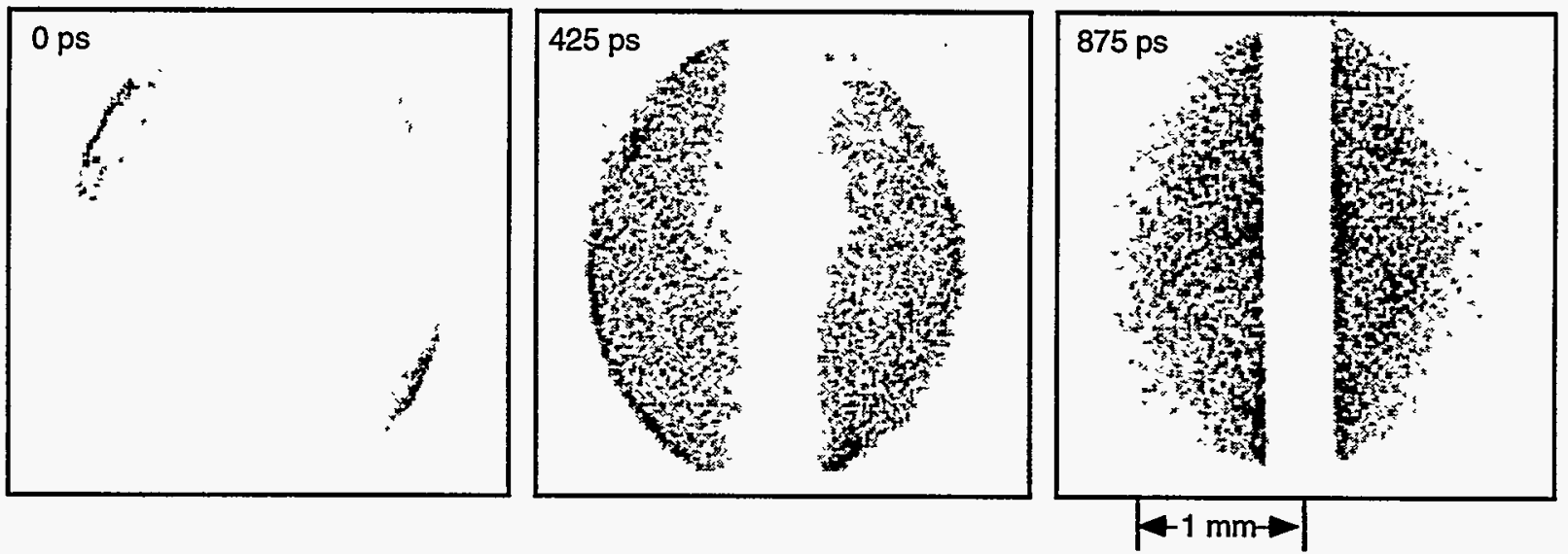

Figure VII-13. Time history of emission of a gasbag, demonstrating the uniformity of a large scalelength plasma. X-ray emission images were taken at three times during the heating pulse, using a gated $x$-ray imager, and represent frame times of $100 \mathrm{ps}$ each. The plasma starts off at laser initiation $(0$ ps) as a glowing emission of the outer shell of the plastic membrane that forms the bag. At $425 \mathrm{~ns}$, after the laser has heated the plasma, it is uniform, with a slight enhancement of emission from the membrane. At 875 ps the gas is extremely uniform. The white strip in the middle of the gasbag is the hoop that holds the plastic membrane together (see Fig. VII-12). This gasbag technique holds much promise with large laser systems like the NIF because scaling of this kind of experiment to large systems is straightforward. 
result is that electrons, and eventually ions, are expelled from the filament, causing the laser light to focus more tightly. This creates an unstable feedback loop whereby a tighter focus leads to higher intensity and more density depletion. Eventually, the instability saturates due to diffraction effects, thermal absorption, or laser light scattering by stimulated Brillouin and Raman scattering instabilities.

The growth of filamentary structures can be determined from the width and length of hot spots, known as speckles, in the incident beam. The filamentation can be described in terms of a growth rate along the length of a speckle; longer speckles are more likely to produce focusing of the hot spot in a filament.

The length of a speckle is given by $8 \mathrm{f}^{2} \lambda$, where $f$ is the $f /$ number of the beam and $\lambda$ is the wavelength of the laser light. Because the length of a speckle is dependent on them, the $\mathrm{f} /$ number and wavelength of the incident laser beam are powerful levers for modifying filamentation, provided that the speckle lengths are smaller than the scale length of the plasma. By using large, uniform plasmas on the NIF, it will be possible to produce very large filaments, allowing the study of filamentation over a wide range of wavelengths and incident-beam $\mathrm{f} /$ numbers.

Understanding the scattering of incident laser light by parametric instabilities and the process of filamentation is of interest to ICF. However, there are many aspects of these instabilities that are not adequately understood and are of interest to scientists for reasons beyond those generated by fusion studies. Understanding of the saturation mechanisms for these instabilities, together with the effects of different types of beam smoothing on filamentation, are subjects that challenge our ability to model these plasmas.

The nonlinear processes that determine saturation of the plasma-wave amplitudes and the way in which depletion of the incident beam limits filamentation and plasma-wave growth are subjects that will benefit from study over a broader range of parameter space (temperatures, densities, and gradient scale lengths) than that expected from ICF targets alone. It is expected that on the NIF it will be possible to extend the parameter space in terms of laser intensity spatial distribution, $f /$ number, pulse length, and wavelength beyond that possible at any existing laser facility. We can explore filamentation over a broad range of experimental parameters by varying the color and $f /$ number of the filamentation beam and by varying the plasma conditions (e.g., temperature, density, and average $Z$ ).

\section{Short-Pulse High-Intensity Laser-Plasma Interactions in Large, Hot Plasmas}

There is widespread agreement that the NIF should include a beam line for short-pulse, highpower experiments. This capability is especially important for many basic plasma-physics studies. These include relativistic, ultrahigh-intensity regimes of laser-matter interaction; generation of astrophysically relevant plasmas; fast-ignitor physics ${ }^{27}$; high-gradient accelerator schemes ${ }^{28}$; new $x$-ray lasers; and high-temporal-resolution diagnostics.

The fast-ignitor laser-fusion concept combines many interesting high-power, shortpulse phenomena. These include generation and transport of high electron fluxes in a high-density plasma, ${ }^{29}$ intense harmonic generation at the critical density, ultrahigh B-fields, ponderomotive effects, relativistic self-focusing and filamentation, laser-beam channeling, and hole boring. 30

A schematic of the fast-ignitor scheme for heating compressed matter is shown in Fig. VII-14. In the first step of the fast-ignitor scheme, an implosion of a sphere filled with gas results in a core of gas with very high densities $(600 \mathrm{~g} / \mathrm{cc})$. Next, a laser beam creates a channel in the sphere by pushing the critical density surface toward the core, after which the final heater or ignitor beam is turned on. The ignitor beam interacts with the density gradient generated by the channeling beam and generates hot electrons at $\mathrm{MeV}$ energies, which penetrate into the compressed gas core and cause an instantaneous rise in the local temperature of the core. The entire concept requires a NIF-like laser because it requires a high compression and two additional laser systems that are both significant. Adding 
A) High-compression implosion

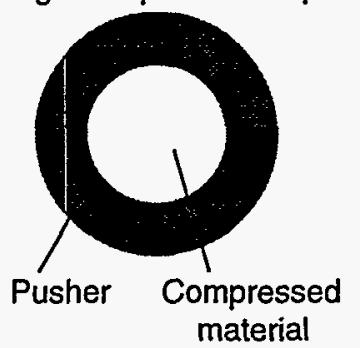

B) Channeling laser beam

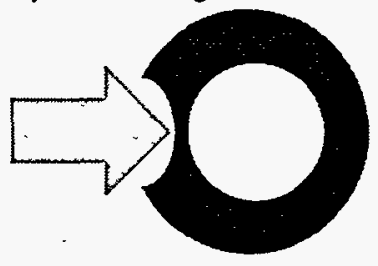

C) Ignitor laser beam

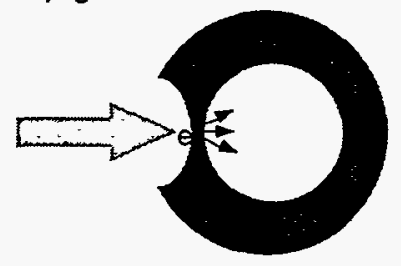

Figure VII-14. Schematic of concept of fast-ignitor scheme for heating. First, in step A there is a highcompression implosion of a sphere filled with gas, resulting in a core of gas that will be at densities of $600 \mathrm{~g} / \mathrm{cc}$. Step B requires that a laser beam with a pulse duration of $100 \mathrm{ps}$ and an intensity of $10^{18}$ $W / \mathrm{cm}^{2}$ create a channel by pushing the critical density surface toward the core. Finally (step $\mathrm{C}$ ), the final heater or ignitor beam is turned on. This beam is a 5-ps beam that has an intensity of $10^{20} \mathrm{~W} / \mathrm{cm}^{2}$. The ignitor beam interacts with the density gradient generated by the channeling beam and generates hot electrons at $\mathrm{MeV}$ energies. These electrons penetrate into the core of the compressed gas and cause an instantaneous rise in the local temperature of the core.

the need to diagnose the system makes clear the need for the NIF in order to perform the experiment.

The high-gradient accelerator schemes involve relativistic self-focusing and filamentation, ${ }^{31}$ and parametric instabilities such as stimulated Brillouin and Raman scattering in strongly driven and transient regimes. ${ }^{32}$

This short-pulse regime is also more amenable to detailed simulation (that is, 2D particle-in-cell codes can be run for times relevant to the entire experiment and include the entire region of interaction)..$^{33}$ It is also more amenable to systematic exploration of linear and nonlinear behavior. Short-pulse experiments would require that a short-pulse beam with the capability for good focusing (i.e., on the order of $20-\mu \mathrm{m}$ diameter) be added to the NIF. These experiments require the NIF in order to produce the large preformed plasma needed to access large interaction lengths.

The high-gradient accelerator schemes use the fact that a plasma can support much higher electromagnetic fields than can conventional accelerators such as those used in elementary particle physics experiments. As a result the highgradient accelerator would be much more compact and potentially cheaper.

A number of novel high-gradient accelerator schemes ${ }^{34}$ have been proposed and studied at laser powers not high enough to produce the desired relativistic electron quiver velocity (velocity induced by the oscillating fields of the laser). Relativistic self-focusing is central to the acceleration scheme to maintain the high laser intensity over many Rayleigh lengths. Other processes such as stimulated Raman and Brillouin scattering or filamentation can break up the laser beam and degrade the efficiency of the scheme. If these high-gradient accelerator schemes prove successful, applications to tunable sources of $x$-rays are also envisioned.

\section{Study of Very High Magnetic Fields}

A further area of focus will be the study of magnetic fields generated by the temperature and density gradients present in plasmas. In laserproduced plasmas the large magnetic fields can be generated in many ways. ${ }^{35}$ For example, one proposed scheme is to generate extremely large magnetic fields by intentionally creating a circulating current path for Raman-generated hot electrons. ${ }^{36}$ This might be done by using a number of beams to irradiate a preformed plasma, such as in a gasbag. Simple estimates show that currents up to $10^{8}$ amperes might be generated using the NIF, and magnetic fields as large as $10^{8}$ gauss could result. Even if the currents and fields are limited to substantially smaller values, this scheme might generate a laser-induced toroidal pinch with intriguing applications. 
Calculations have indicated that magnetic fields generated by laser-produced plasmas can be as large as 100 MG. ${ }^{37}$ We may be able to use Faraday rotation of an optical probe beam and Zeeman splitting of spectral lines to investigate and quantify these magnetic fields. By inhibiting thermal conduction, magnetic fields influence temperature distributions and thereby influence stimulated Brillouin and Raman scattering, filamentation, and propagation of laser beams in plasmas. We may also be able to study the effect of magnetic fields on atomic energy levels by means of simultaneous $x$-ray spectroscopy. One interesting possibility is to use the Weibel instability driven by intentionally induced anisotropy in the electron velocity distribution.

These experiments would require the NIF in order to create significant (and hence diagnosable) volumes of plasma at the same time that extremely large magnetic fields are generated.

\section{Generation of Large Fluxes of High-Energy Electrons (50-100 keV)}

On the NIF it would be possible to study the deliberate production of fast electrons through stimulated Brillouin scattering and two-plasmon decay mechanisms in plasmas that are at 0.1 to 0.25 of the critical density. ${ }^{38}$ In particular, these plasmas could be preformed from gas-filled targets or foams.

It would also be possible to study the conversion of high-energy electrons $(50-100 \mathrm{keV})$ to $x$-rays. Appropriate high- $Z$ layers would be placed near the target. The fast electrons could then deposit their energy in the high- $Z$ material and the resultant $x$-ray emission would be studied.

It might be possible to develop a compact high-energy $x$-ray source that could be used for backlighting/probing of other experiments.

Finally, the enormous currents created by these methods would generate magnetic fields, and the study of these magnetic fields would also be of interest-see Section IX, Radiative Properties for a discussion of strong magnetic field effects.

\section{Interpenetrating Plasmas and Turbulence}

The study of the interaction, or interpenetration, of two high-temperature plasmas has received limited attention experimentally. ${ }^{39}$ Apart from its intrinsic interest, the interaction of plasma streams is part of the standard ICF experiments because of the generation of plasma motion inside a hohlraum. One particular interest is the equipartition of energy between the two groups of ions, and in this regard it has been calculated that ions can achieve very high temperature through collisions.

Moreover, the inter-streaming plasmas can provide the ideal system for generating and studying turbulence in plasmas. This would make it possible to study the generation of twostream ion-ion turbulence or the ion Weibel instability. The ion Weibel instability is of interest because it creates magnetic fields with $\lambda \sim c / \omega_{p i}$ but has a slow growth rate of $\sim \omega_{p i} u / c$, where $\omega_{p i}$ is the ion plasma frequency and $u$ is the relative velocity of the interpenetrating flows.

Producing high-temperature plasmas that can collide with sufficiently high density for interactions to take place requires large amounts of laser energy. Nova and other present-day laser systems are severely limited in the amount of laser energy available. Current laser facilities are also not capable of creating plasmas large enough to permit using diagnostics to probe the region of interpenetration with sufficient spatial resolution. Although some preliminary work could be performed on present-day lasers, the energies available in the NIF will make these studies far more attractive, with quantitative results being feasible.

The study of ion turbulence will require a diagnostic such as Thomson scattering that will permit the direct measurement of $\Delta n / n$ of the ions as a function of time. The diagnostic should allow this measurement to be made in different directions, thus permitting us to follow the randomization of the ion fluctuations as well as the anisotropy of ion acoustic properties. The NIF, with the much larger plasmas it will be able to generate, will make these kinds of diagnostics possible. 


\section{Nuclear Reactions in Large-Volume, High-Temperature Plasmas}

A great deal of study has been done to characterize the behavior of ICF capsules. As discussed earlier in Section IV, Astrophysics and Space Physics, these capsules are expected to achieve conditions suitable for testing certain nuclear reaction rates of astrophysical interest. However, compressional heating of ICF-like capsules may not be the only way to explore nuclear reaction rates at temperatures corresponding to stellar interiors. We note that most Main Sequence stars have core temperatures between 1 and $2 \mathrm{keV}$ and that Nova experiments are already raising millimeter-size gas samples to temperatures near $2 \mathrm{keV}$.

While the temperatures and densities of gasbag experiments are lower than what might be achieved in an imploded capsule (such as an ICF capsule), they are sufficient to examine many light-element reaction rates, and there are definite advantages to direct heating of gas samples. With direct heating, the large sample size guarantees homogeneous conditions that can be held for relatively long (nanosecond) periods. This eliminates much of the difficulty and uncertainty in converting an observed number of reactions into an energy-dependent cross section. With 60 times the energy, the NIF will be capable of raising larger samples to higher temperatures.

An example of a reaction rate that might be studied in this way is the ${ }^{3} \mathrm{He}+{ }^{3} \mathrm{He} \rightarrow 2 \mathrm{p}+{ }^{4} \mathrm{He}$ reaction. This reaction rate is important for the branching ratio between the branches of the proton-proton chain (PPI and PPII) in the sun, and therefore has direct consequences on the expected neutrino rate. Table VII-1 gives the number of reactions expected in a nanosecond from a $0.6 \mathrm{~cm}^{3}$ gas sample at $3 \mathrm{~atm}$.

Another important cross section for astrophysics involves the destruction of ${ }^{7} \mathrm{Li}$. Primordial lithium abundances are a critical constraint on big-bang nucleosynthesis and cosmology. Typically the primordial abundances are obtained from the lithium abundances seen in the oldest stars. However, the observed abundances must be corrected for any destruction that occurs, and observations of old clusters indicate that more lithium is destroyed than expected. The main reaction for this destruction, ${ }^{7} \mathrm{Li}+\mathrm{p} \rightarrow{ }^{8} \mathrm{Be} \rightarrow 2{ }^{4} \mathrm{He}$, is more than 5000 times larger than the ${ }^{3} \mathrm{He}$ rate at $2.1 \mathrm{keV}$. Table VII-2 shows other reactions that have rates expected to be higher than the ${ }^{3} \mathrm{He}+{ }^{3} \mathrm{He}$ rate at $2.1 \mathrm{KeV}$, and which are thus candidates for investigations on the NIF.

Table VII-1. The number of reactions for ${ }^{3} \mathrm{He}$ $+{ }^{3} \mathrm{He}$ in a $0.6-\mathrm{cm}^{3} 3-\mathrm{atm}$ sample.

\begin{tabular}{cc}
\hline Temperature (keV) & Reactions per ns \\
\hline 2.1 & 2 \\
2.6 & 16 \\
3.4 & 280 \\
4.3 & 5500 \\
\hline
\end{tabular}

Table VII-2. Selected reactions having rates larger than the ${ }^{3} \mathrm{He}+{ }^{3} \mathrm{He}$ rate at $2.1 \mathrm{keV}$.

\begin{tabular}{rll}
\hline $\mathrm{D}+\mathrm{p}$ & $\rightarrow$ & ${ }^{3} \mathrm{He}+\gamma$ \\
$\mathrm{D}+\mathrm{D}$ & $\rightarrow$ & $\mathrm{n}+{ }^{3} \mathrm{He}$ \\
$\mathrm{D}+\mathrm{D}$ & $\rightarrow$ & $\mathrm{p}+\mathrm{T}$ \\
$\mathrm{T}+\mathrm{T}$ & $\rightarrow$ & ${ }^{2} \mathrm{n}+{ }^{4} \mathrm{He}$ \\
${ }^{3} \mathrm{He}+\mathrm{D}$ & $\rightarrow$ & $\mathrm{p}+{ }^{4} \mathrm{He}$ \\
${ }^{3} \mathrm{He}+\mathrm{T}$ & $\rightarrow$ & $\mathrm{D}+{ }^{4} \mathrm{He}$ \\
${ }^{3} \mathrm{He}+\mathrm{T}$ & $\rightarrow$ & $\mathrm{n}+\mathrm{p}+{ }^{4} \mathrm{He}$ \\
${ }^{4} \mathrm{He}+\mathrm{T}$ & $\rightarrow$ & ${ }^{7} \mathrm{Li}+\gamma$ \\
${ }^{6} \mathrm{Li}+\mathrm{p}$ & $\rightarrow$ & ${ }^{7} \mathrm{Be}+\gamma$ \\
${ }^{6} \mathrm{Li}+\mathrm{p}$ & $\rightarrow$ & ${ }^{4} \mathrm{He}+{ }^{3} \mathrm{He}$ \\
${ }^{7} \mathrm{Li}+\mathrm{D}$ & $\rightarrow$ & $\mathrm{n}+2{ }^{4} \mathrm{He}$ \\
${ }^{7} \mathrm{Li}+\mathrm{T}$ & $\rightarrow$ & ${ }^{2} \mathrm{n}+2^{4} \mathrm{He}$ \\
${ }^{9} \mathrm{Be}+\mathrm{p}$ & $\rightarrow$ & $\mathrm{D}+2^{4} \mathrm{He}$ \\
${ }^{9} \mathrm{Be}+\mathrm{p}$ & $\rightarrow$ & ${ }^{4} \mathrm{He}+{ }^{6} \mathrm{Li}$ \\
${ }^{10} \mathrm{~B}+\mathrm{p}$ & $\rightarrow$ & ${ }^{4} \mathrm{He}+{ }^{7} \mathrm{Be}$ \\
${ }^{11} \mathrm{~B}+\mathrm{p}$ & $\rightarrow$ & $3^{4} \mathrm{He}$ \\
\hline
\end{tabular}




\section{E. References}

1. See the discussion in H. A. Baldis, E. M. Campbell, and W. L. Kruer, "Laser Plasma Interactions," Handbook of Plasma Physics, M. N. Rosenbluth and R. Z. Sagdeev, Eds., Volume 3: Physics of Laser Plasmas, A. M. Rubenchik and S. Witkowski, Eds. (Elsevier Science Publishers, B. V., 1991).

2. E. Ott and W. M. Mannheimer, Nuclear Fusion 17, 1057 (1977).

3. D. J. Southwood, Planet. Space Sci. 16, 587 (1968).

4. L. L. Smarr, M. L. Norman, and K. A. Winkler, Physica 12D 83 (1984).

5. T. A. Peyser et al., Phys. Fluids B 42, 448 (1992).

6. Handbook of Plasma Physics, M. N. Rosenbluth and R. Z. Sagdeev, Eds., Volume 3: Physics of Laser Plasmas, A. M. Rubenchik and S. Witkowski, Eds. (Elsevier Science Publishers, B. V., 1991), Chapter 9 and references therein.

7. F. Ze, L. J. Suter, S. M. Lane, E. M. Campbell, W. C. Mead, J. D. Lindl, M. D. Rosen, D. W. Phillion, C. W. Hatcher, R. P. Drake, J. S. Hildum, and K. R. Manes, Comments Plasma Phys. and Controlled Thermonuclear Fusion 10, 33 (1986).

8. D. Montgomery and E. M. Campbell, Comments Plasma Phys. and Controlled Thermonuclear Fusion (1990).

9. E. M. Campbell in: Radiation in Plasmas, B. McNamara, Ed., Volume I (1984), pp. 579621.

10. K. R. Manes, V. C. Rupert, J. M. Auerbach, P. Lee, and J. E. Swain, Phys. Rev. Lett. 39, 281 (1977).

11. A. G. M. Maaswinkel, K. Eidmann, and R. Sigel, Phys. Rev. Lett. 42, 1625 (1979).

12. R. L. Carman, C. K. Rhodes, and R. F. Benjamin, Phys. Rev. A 24, 2649 (1981).

13. H. A. Baldis and C. J. Walsh, Phys. Fluids 26, 3426 (1981).

14. H. A. Baldis, J. C. Samson, and P. B. Corkum, Phys. Rev. Lett. 41, 1719 (1978).

15. M. D. Rosen, D. W. Phillion, V. C. Rupert, W. C. Mead, W. L. Kruer, J. J. Thomson, H. N. Kornblum, and M. N. Rosenbluth, Phys. Rev. Lett. 29, 565 (1979).
16. J. E. Bernard and J. Mayer, Phys. Fluids 29, 2313 (1986).

17. C. J. Walsh and H. A. Baldis, Phys. Rev. Lett. 48, 1483 (1982).

18. J. D. Moody, H. A. Baldis, D. S. Montgomery, K. Estabrook, S. Dixit, and C. Labaune, Journal of Fusion Energy 12, 323 (1993).

19. C. J. Walsh, D. M. Villeneuve, and H. A. Baldis, Phys. Rev. Lett. 53, 1445 (1984).

20. H. A. Baldis, R. P. Drake, W. L. Kruer, K. G. Estabrook, E. A. Williams, and T. W. Johnston, Phys. Rev. Lett. 62, 2829 (1989).

21. C. Joshi, C. E. Clayton, A. Yasuda, and F. F. Chen, J. Appl. Phys. 53, 215 (1982).

22. J. A. Stamper, R. H. Lehmberg, A. Schmitt, M. J. Herbst, E. C. Young, J. H. Gardner, and S. P. Obenschain, Phys. Fluids 28, 2563 (1985).

23. P. E. Young, H. A. Baldis, R. P. Drake, E. M. Campbell, and K. G. Estabrook, Phys. Rev. Lett. 61, 2336 (1988).

24. For stimulated Raman scattering see: W. Seka et al., Phys. Fluids 27, 2181 (1984); R. P. Drake, Phys. Fluids B 1, 1082 (1989); R. P. Drake and R. E. Turner, in Laser Interaction \& Related Plasma Phenomena, Volume 9, H. Hora and G. H. Miley, Eds. (1991); K. Estabrook, W. L. Kruer, and B. F. Lasinski, Phys. Rev. Lett. 45, 1399 (1980); S. P. Batha et al., Phys. Rev. Lett. 66, 2324 (1991); R. E. Turner et al., Phys. Rev. Lett. 57, 1725 (1986).

25. For stimulated Brillouin scattering see P. E. Young, Phys. Fluids B 3, 1245 (1991) and the references therein.

26. P. E. Young, Phys. Rev. Lett. 61, 2336 (1988); C. Labaune et al., Phys. Fluids B 4, 2224 (1992).

27. M. Tabak, J. Hammer, M. E. Glinsky, W. L. Kruer, S. C. Wilks, J. Woodworth, E. M. Campbell, and M. D. Perry, Phys. Plasmas 1, 1626 (1994).

28. J. M. Dawson, Sci. Am. 260, No. 3, 54 (1989); "Advanced Accelerator Concepts," J. S. Wurtele, Ed., AIP Conf. Proc. No. 279 (AIP, NY, 1993).

29. M. E. Glinsky, R. J. Mason, and M. Tabak, Bull. Am. Phys. Soc. 38, 2080 (1993).

30. S. C. Wilks, W. L. Kruer, M. Tabak, and A. B. Langdon, Phys. Rev. Lett. 69, 1383 (1992); X. Liu and D. Umstadter, Phys. Rev. Lett. 69, 
1935 (1992); M. P. Kalashnikov, P. V. Nichels, Th. Schlegel, M. Schnuerer, F. Billhardt, I. Will, W. Sandner, and N. N. Demchenko, Phys. Rev. Lett. 73, 260 (1994).

31. P. Sprangle et al., Appl. Phys. Lett. 53, 2146 (1988).

32. T. Antonsen and P. Mora, Phys. Rev. Lett. 69, 2204 (1992); S. C. Wilks et al., Phys. Rev. Lett. 69, 1383 (1992); W. B. Mori et al., Phys. Rev. Lett. 72, 1482 (1994).

33. M. E. Glinsky, R. J. Mason, and M. Tabak, Bull. Am. Phys. Soc. 38, 2080 (1993).

34. C. E. Clayton, K. A. Marsh, A. Dyson, M. Everett, A. Lal, W. P. Leemans,
R. Williams, and C. Joshi, Phys. Rev. Lett. 70, 37 (1993) and references therein.

35. M. G. Haines, Can. J. Phys. 64, 912 (1986); this contains a review of the different ways of generating B-fields in a plasma.

36. W. L. Kruer, "Laser Induced Circulating Currents," to be published (1994), in Bull. Am. Phys. Soc., DPP meeting 1994, Minneapolis, Minnesota.

37. S. C. Wilks, W. L. Kruer, M. Tabak, and A. B. Langdon, Phys. Rev. Lett. 69, 1383 (1992).

38. For two-plasmon decay see H. A. Baldis and C. J. Walsh, Phys. Fluids 26, 1364 (1983);

A. Simon et al., Phys. Fluids 26, 3107 (1983).

39. R. Bosch et al., Phys. Fluids B 4, 979 (1992). 
This page intentionally left blank 


\section{Section VIII}

\section{Radiation Sources}

\section{A. Spectrally Continuous Sources}

There are two broad categories of quasicontinuous radiation sources. First there is the radiation source generated by a hohlraum and used to heat a sample either inside the hohlraum (as in the case of an implosion microsphere or an opacity sample), or abutted to it (as with the radiation-flow experiments or the hydrodynamic mixing interface experiments). With this we group the gold burn-through foils used to heat the non-local thermodynamic equilibrium (NLTE) tamped samples. Second, we have the effort to develop backlights that are quasicontinuous.

In the first category the work done on the gold will be used to illustrate the cogent features. These burn-through foils have been studied in detail and provide a good sense of what to expect from very similar processes that occur in a hohlraum, noting, of course, that in a hohlraum one is using the front surface emission and an enclosure that will increase the energy in the radiation field.

\section{Conversion Efficiency of Gold Burn-Through Foils}

The efficiency with which gold burn-through foils convert visible laser light to $x$-rays can be roughly quoted as being in the region of $\geq 40 \%$ of the laser energy on target, noting that many papers use a conversion efficiency of $x$-ray energy to absorbed laser energy. We choose the former method because it gives a better idea of the $x$-rays available from a particular laser.

Figure VIII- 1 shows the conversion efficiency from the front and rear sides for gold foils of varying thicknesses, for the two laser intensities of $10^{12}$ and $10^{13} \mathrm{~W} / \mathrm{cm}^{2} \cdot{ }^{1}$ Conversion efficiency is observed from both the front and rear surfaces. Efficiency observing from the front is shown in Fig. VIII-1 as data points rising with increased thickness, and from the rear is shown as data points falling with increasing foil thickness. The limiting case of a solid target is approximated by a $10,000-\AA$ foil, where the conversion efficiency transmitted in $x$-rays is very small, while the front-side conversion rises to $\sim 50 \%$ or more. Note that these data have been obtained both with filtered $x$-ray diode arrays and with spectrometers, and both data sets are shown.

Figure VIII-1 illustrates two important points. First, the conversion efficiency is not strongly dependent on intensity above $10^{12}$ $\mathrm{W} / \mathrm{cm}^{2}$, and second, the thinnest foils convert less. The reason for this is obvious as the transmission of laser energy through the foil increases dramatically as the foil becomes thin. The transmission is presented in Fig. VIII-2, where the ratio of the transmitted laser energy to incident laser energy is shown. Note that in the figure the higher intensity shows more burnthrough, but the fraction is less than or equal to 0.01 for $1500-\AA$ foils. This burn-through is due to inhomogeneities-hot spots or intensity fluctuations - in the beam profile. These hot spots can be smoothed out and, indeed, have been in more recent experiments.

\section{Spectral Character of Gold Burn-Through Foils}

The spectral character of the emission found from a gold target similar to ones used in Fig. VIII-1 is shown in Fig. VIII-3. ${ }^{2}$ Here the timedependent spectrum is shown vs energy. The spectrum was taken from the rear side of a gold 


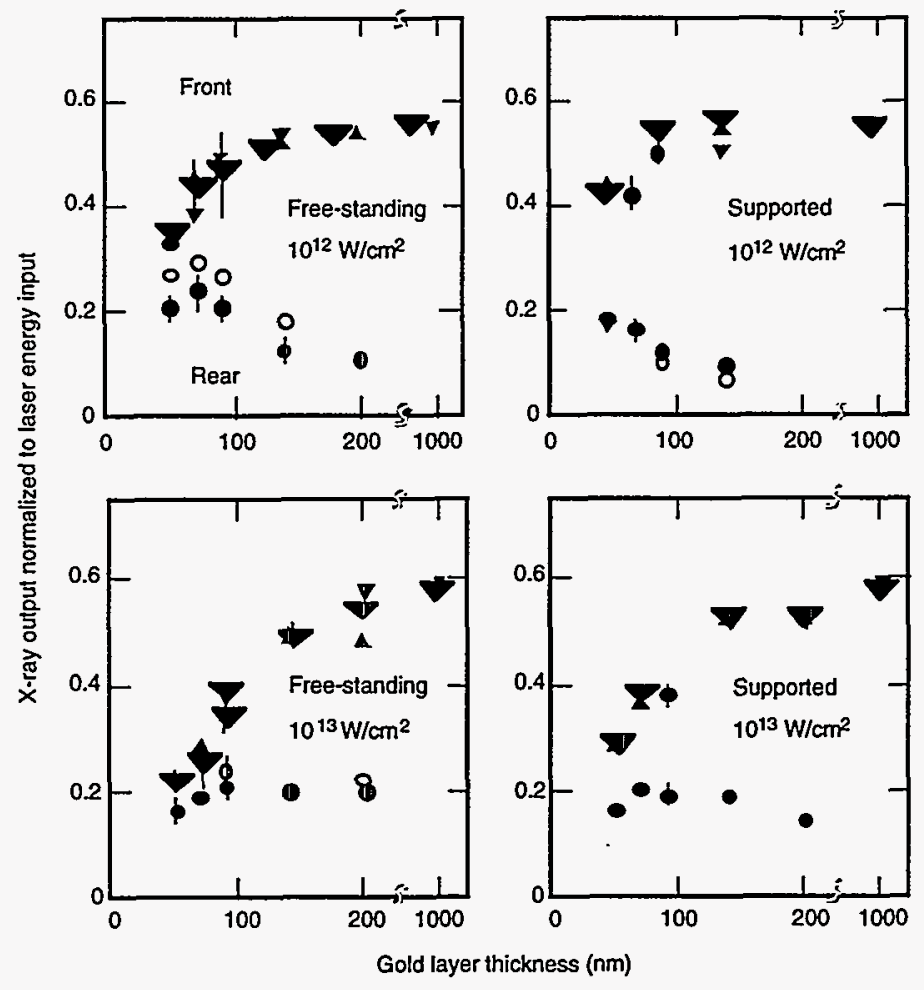

Figure VIII-1. Total $x$-ray emission, measured as conversion efficiency vs target thickness, from the front and rear surfaces of free-standing and supported gold targets. The gold foils vary in thickness from $500 \AA$ to $10,000 \AA$, and irradiation is $10^{12}$ and $10^{13} \mathrm{~W} / \mathrm{cm}^{2}$. The front surface always has a larger conversion efficiency than the rear. Note that the method of support and the intensity do not affect the results.

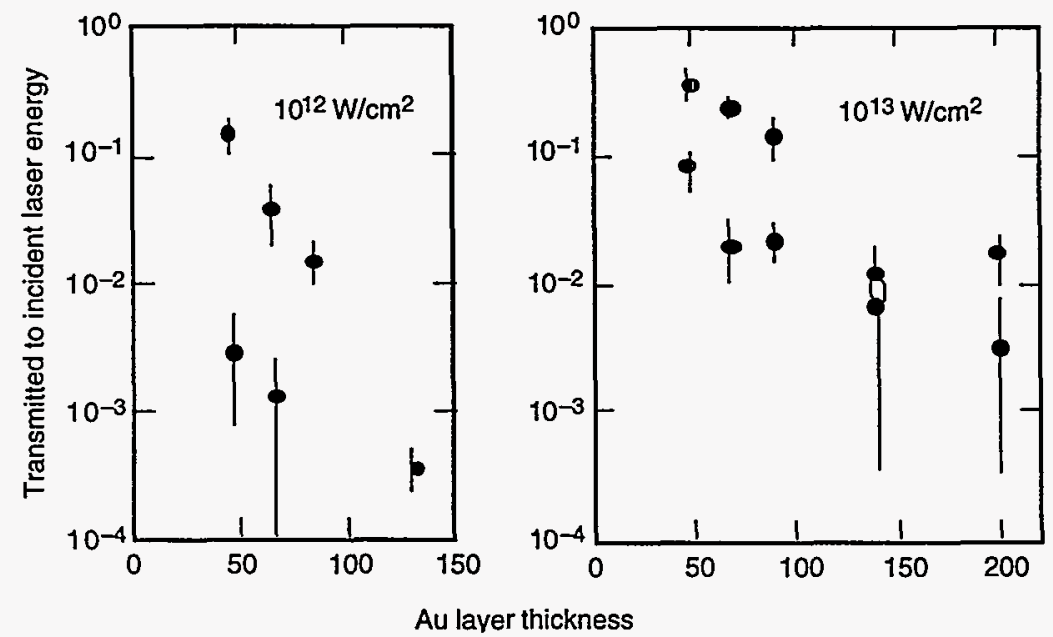

Figure VIII-2. Measurement of transmitted laser energy as a fraction of incident laser energy vs target thickness, for two different intensities. This is from the same experimental setup used to obtain the data in Fig. VIII-1. 

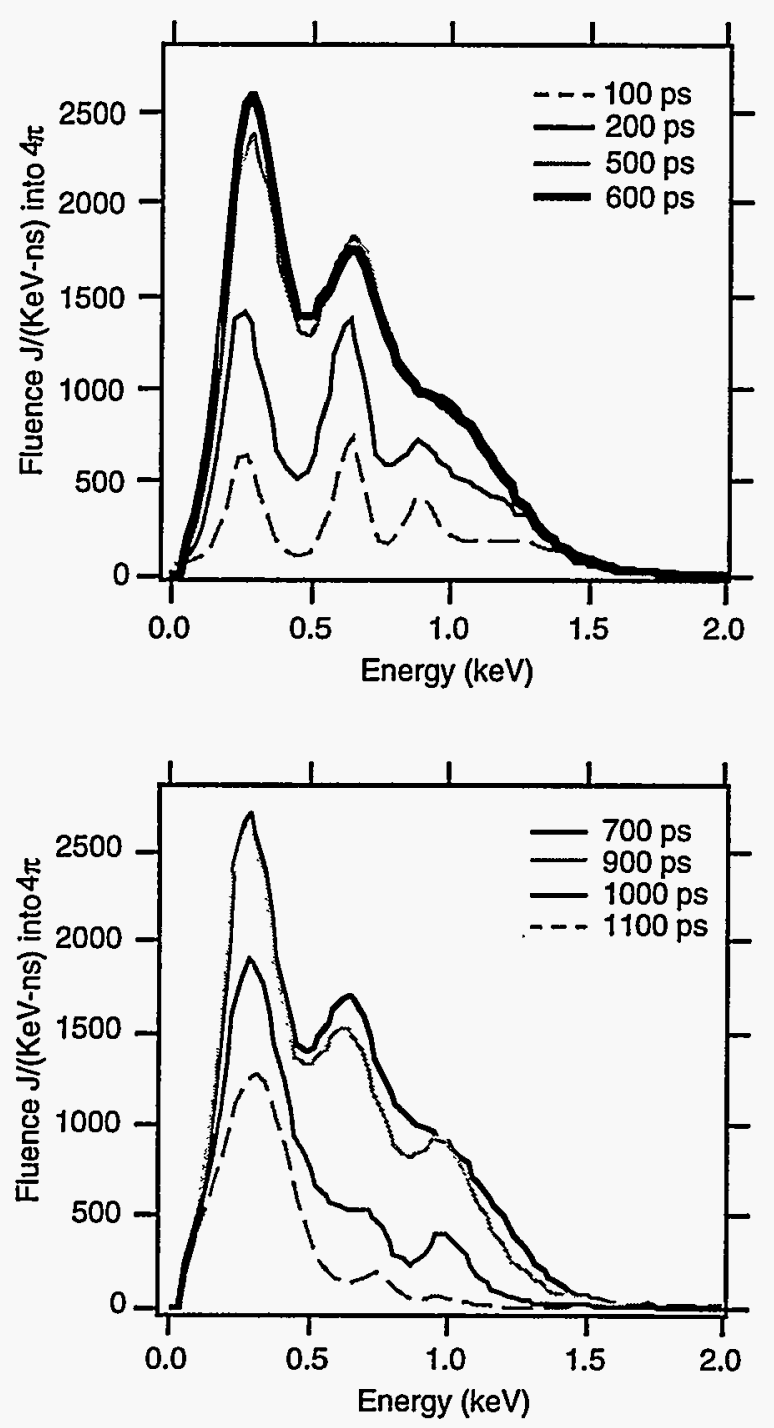

Figure VIII-3. Time history of spectrum vs energy in $\mathrm{eV}$ from the rear side of a 1000-Á gold foil supported on a $5000-\dot{A}$ plastic layer irradiated at $2 \times 10^{14} \mathrm{~W} / \mathrm{cm}^{2}$. (The front and back spectra of a target of this thickness will be very similar, with no major differences in the spectral character.) The results were measured by a 12-channel filtered $x$-ray diode that covers the range from 80 through $2000 \mathrm{eV}$.

foil that was $1000 \AA$ thick, mounted on a $5000-\AA$ layer of plastic. (The front and back spectra of a target of this thickness will be very similar, with no major differences in the spectral character.) The location of the two "bumps" in the spectrum correspond to the oxygen and nitrogen bands, at $\sim 300 \mathrm{eV}$ and $\sim 800 \mathrm{eV}$ respectively.
There is also an M-band contribution, which does not show up on soft $x$-ray instruments because it spans the energies between $2000 \mathrm{eV}$ and $3600 \mathrm{eV}$. Figure VIII- 4 shows the M-band contribution for a gold disk irradiated at $2 \times 10^{14} \mathrm{~W} / \mathrm{cm}^{2}{ }^{3}$ This band-like structure is obtained for all high- $Z$ elements. The line drawn on the figure indicates the spectral region over which the $\mathrm{M}$-band contribution is defined.

\section{Temporal Behavior of Gold Burn-Through Foils}

The time history of the emission in the harder $x$-ray regimes, such as the M-band, will follow the laser pulse, and there is much data to support this supposition. However, the soft $\mathrm{x}$-ray spectrum will last for time longer than the laser pulse, and duration will increase as the wavelength of the radiation increases. Thus, for the softest $x$-rays measured using the filtered $x$-ray diodes in Fig. VIII-3 (i.e., $\sim 80 \mathrm{eV}$ ), the time duration will be two to three times the pulse duration. Because the pulse is $1 \mathrm{~ns}$ in duration, this indicates emission on the order of several ns.

In Fig. VIII-5, the total energy emitted per unit time from a source on the rear of gold foils of various thicknesses is shown. The foils were irradiated at $2 \times 10^{14} \mathrm{~W} / \mathrm{cm}^{2}$. Normalization is made to a sphere (i.e., "into $4 \pi^{\prime \prime}$ ), but the measurements reflect only the intensity of a specific ray. Thus, the correct units should be joules per ns per steradian, and the fact that these sources tend to look Lambertian (i.e., they have $\cos (\varnothing)$ dependence) provides the correct intensity. Here $ø$ is the angle of observation relative to the target surface normal.

\section{Spectral Character of Other Foils}

Next we illustrate the fact that the spectral character of the soft $x$-ray emission can be modified by the choice of target material. 4 Figure VIII- 6 A) shows the soft $x$-ray spectrum from pure targets of several elements from $Z=56$ to $Z=63$, while $B$ ) shows the soft $x$-ray spectrum from targets consisting of a compound that contains approximately $1 \%$ of the same element.

The spectrum in each pair of cases shows that as the opacity of the sample is increased by using 


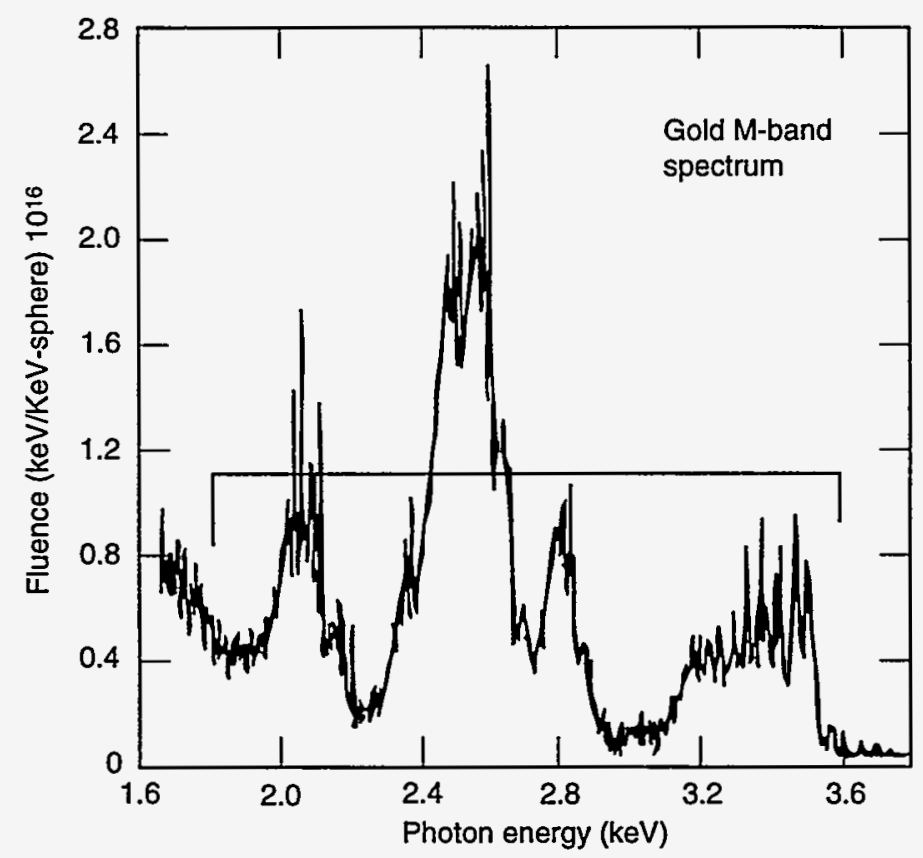

Figure VIII-4. Spectrum of the gold M-bands, which cover the spectral region from 2000 to $3600 \mathrm{eV}$, from a thick target with intensities of $2 \times 10^{14} \mathrm{~W} / \mathrm{cm}^{2}$. This band-like structure is obtained for all high$\mathrm{Z}$ elements. The line indicates the spectral region over which the $\mathrm{M}$-band contribution is defined. The units used here are unique to the inertial confinement fusion (ICF) program at LLNL and can be easily converted.

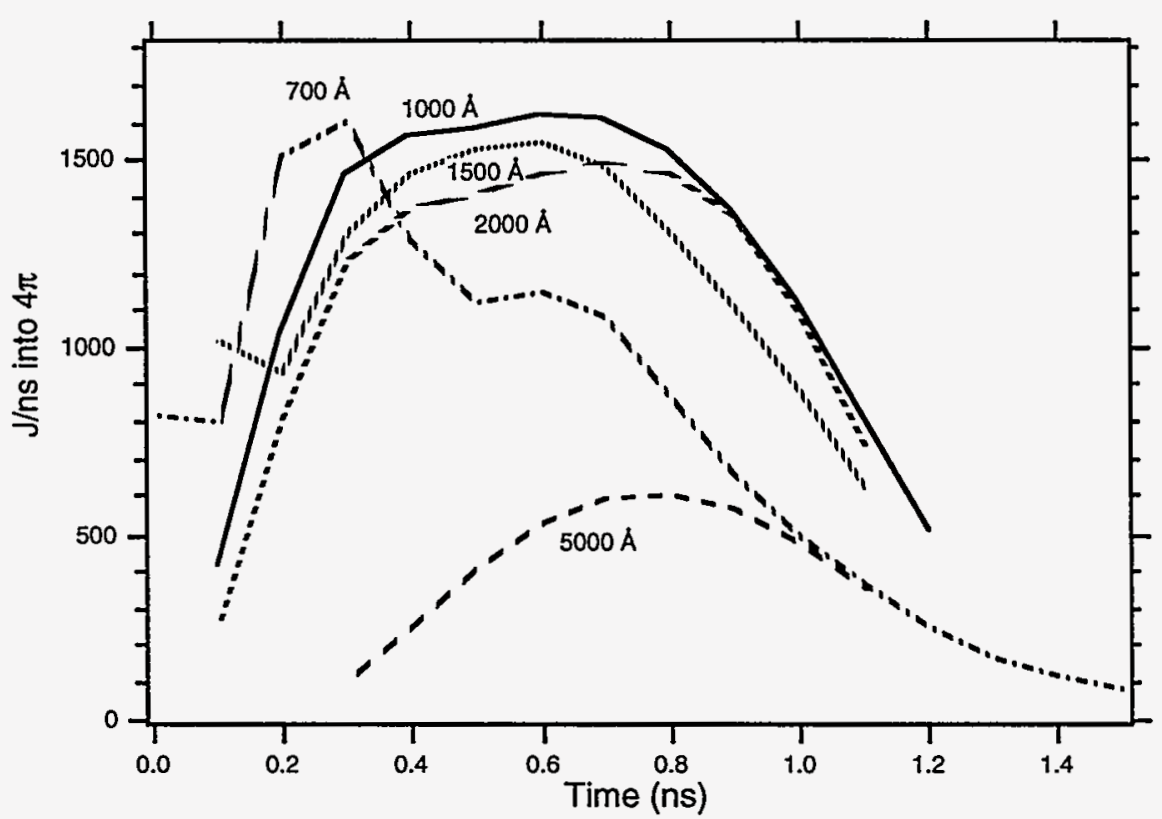

Figure VIII-5. Temporal history of spectrally integrated emission from the rear side of gold foils, for various foil thicknesses irradiated at $2 \times 10^{14} \mathrm{~W} / \mathrm{cm}^{2}$. Note that the duration of the laser, $1 \mathrm{~ns}$ in this case, is not closely followed by the softer $x$-ray emission. The time delay for the thicker foils occurs due to the lag in getting the softer $x$-ray to the back surface. Also note that the hard $x$-ray flux (e.g., the gold M-band contribution) shows no timing differences with different foil thickness. 


\section{A) Pure element}

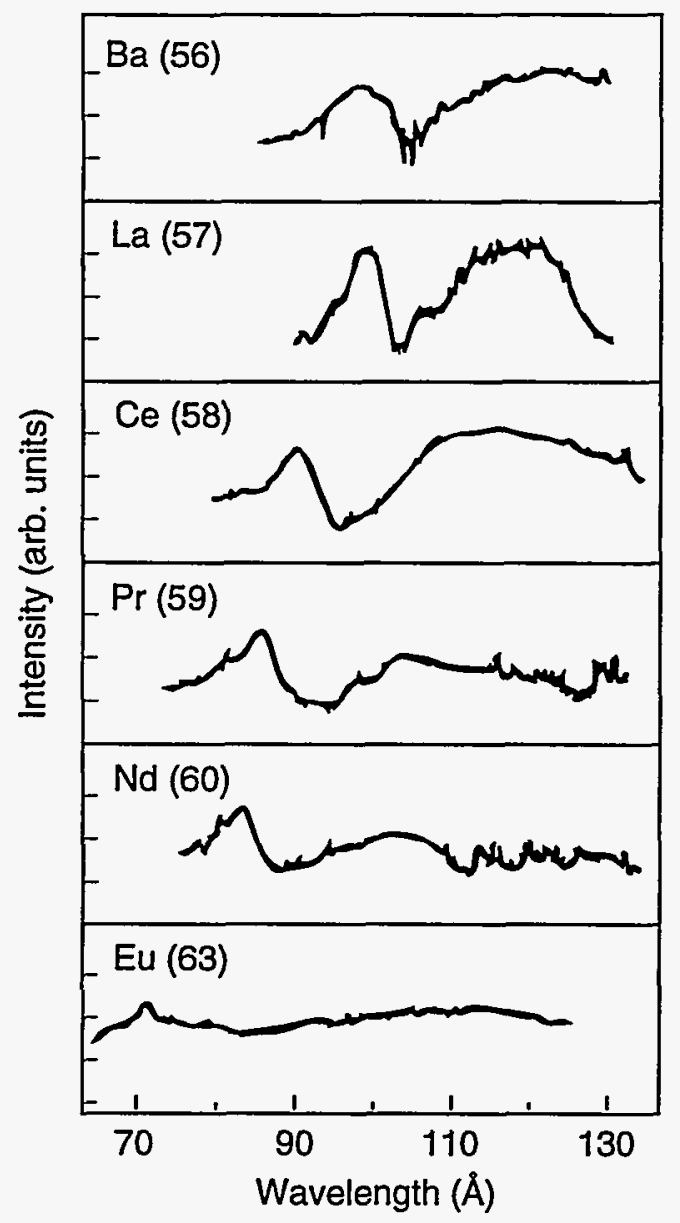

B) Element with $1 \%$ impurity

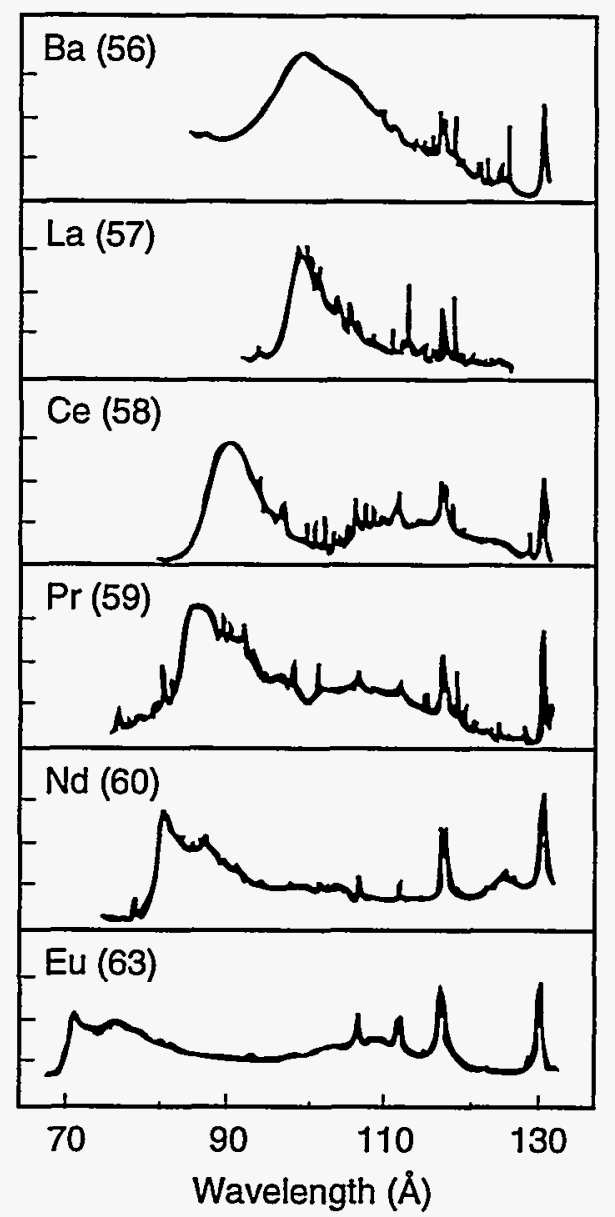

Figure VIII-6. Spectral character of various elements from $Z=56$ to $Z=63$. In cases $A$ ) the targets are made of the pure element, while in cases B) the element is a $1 \%$ impurity in the target material. The increased optical depth in the cases with the pure element leads to an enhancement of the soft $x$-ray features compared to the cases with the impurity, where the optical depth is low and the evidence of individual lines becomes apparent. Note also that the dominant features move toward higher energy as $\mathrm{Z}$ increases.

a pure $\mathrm{Z}$ target, the softer $\mathrm{x}$-ray feature and thereby the quasi-continuous nature of the emission are increased. Moreover, the ability to tailor the source in energy by changing $Z$ can be observed.

Figure VIII-7 shows the same type of spectral data as does Fig. VIII-6, but for a much wider range of Zs. ${ }^{5}$ The figure shows the front-surface spectra measured in $10^{12}$ ergs per $\mathrm{cm}$ per steradian for an irradiance of $3 \times 10^{13} \mathrm{~W} / \mathrm{cm}^{2}$.
The experiments used the frequency-doubled laser at a wavelength of $0.53 \mu \mathrm{m}$ and pulse length was 3 ns. Note that the gold, oxygen, and nitrogen bands are seen while the $M$ band is beyond the spectral coverage.

Figure VIII-8 shows the absolute conversion efficiency of $x$-ray production in absolutely measured spectra emitted by laser-irradiated targets as a function of target $Z$. Note that the net increase in $x$-ray conversion is quite marked for the high-Z elements. 

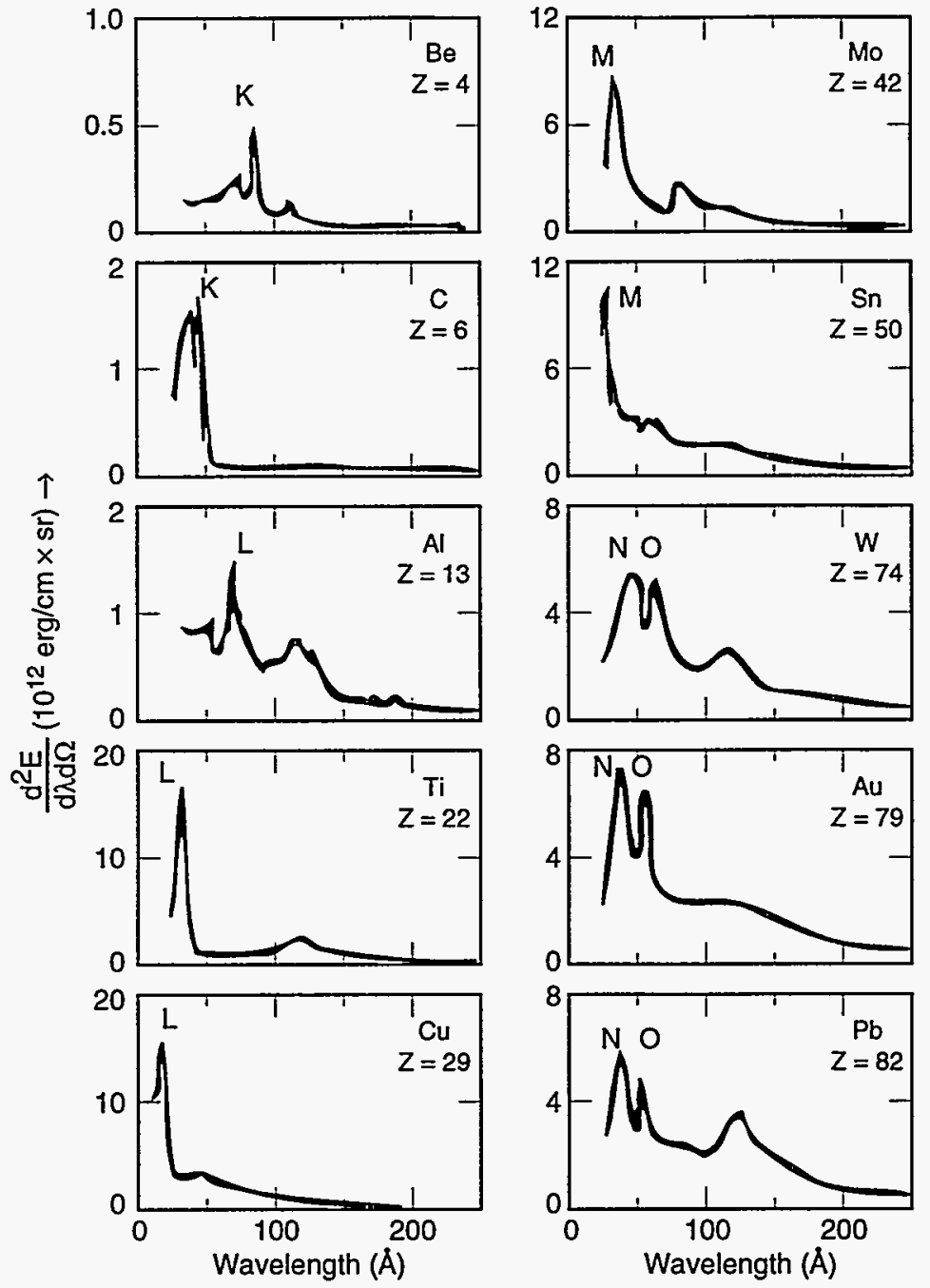

Figure VIII-7. Absolutely measured spectra emitted by laser-irradiated targets of different elements. Intensity is $3 \times 10^{13} \mathrm{~W} / \mathrm{cm}^{2}$, pulse duration is $3 \mathrm{~ns}$, and wavelength of the laser is $0.53 \mu \mathrm{m}$. The spectroscopic structure $K, L, M, N$, and $O$ refer to the shells from which the emission arises. Note that the intensity axis changes in the various plots.

\section{Angle of Incidence vs Conversion Efficiency}

The next pertinent issue concerns the effect that the angle of incidence of the laser with respect to the target surface normal has on conversion efficiency. There are two obvious geometries that can be used to irradiate a sample with the $x$-rays from the laser-conversion target.

The first is to place the sample at the rear of a high- $Z$ foil that is thin enough to let $x$-rays through, but thick enough to stop laser light from being transmitted (see Figs. VIII-1 and VIII-2 for information on effects of target thickness). The second is an angled arrangement used to enhance radiation, based on the fact that the front-surface $x$-ray conversions are higher. For this second geometry, the effect of the angle of incidence is important.

Studies have been made of the effect of angle of incidence on conversion efficiency. In Fig. VIII-9 we show two quantities as a function of angle of incidence of the laser. ${ }^{6}$ First, the lefthand axis refers to absorption of the laser light by 


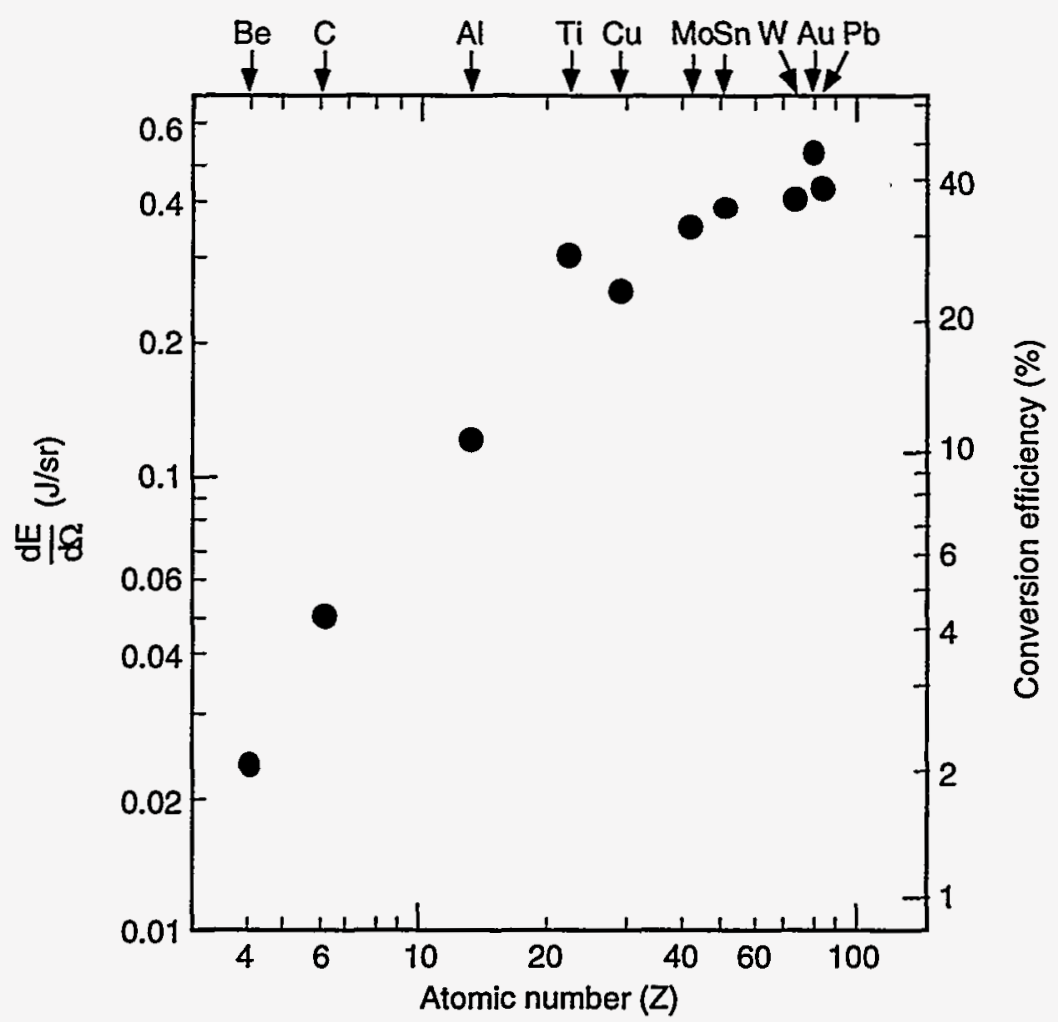

Figure VIII-8. Absolute conversion efficiency of laser-irradiated targets of different elements from beryllium through lead. Net increase in $X$-ray conversion is quite marked for the high-Z elements.

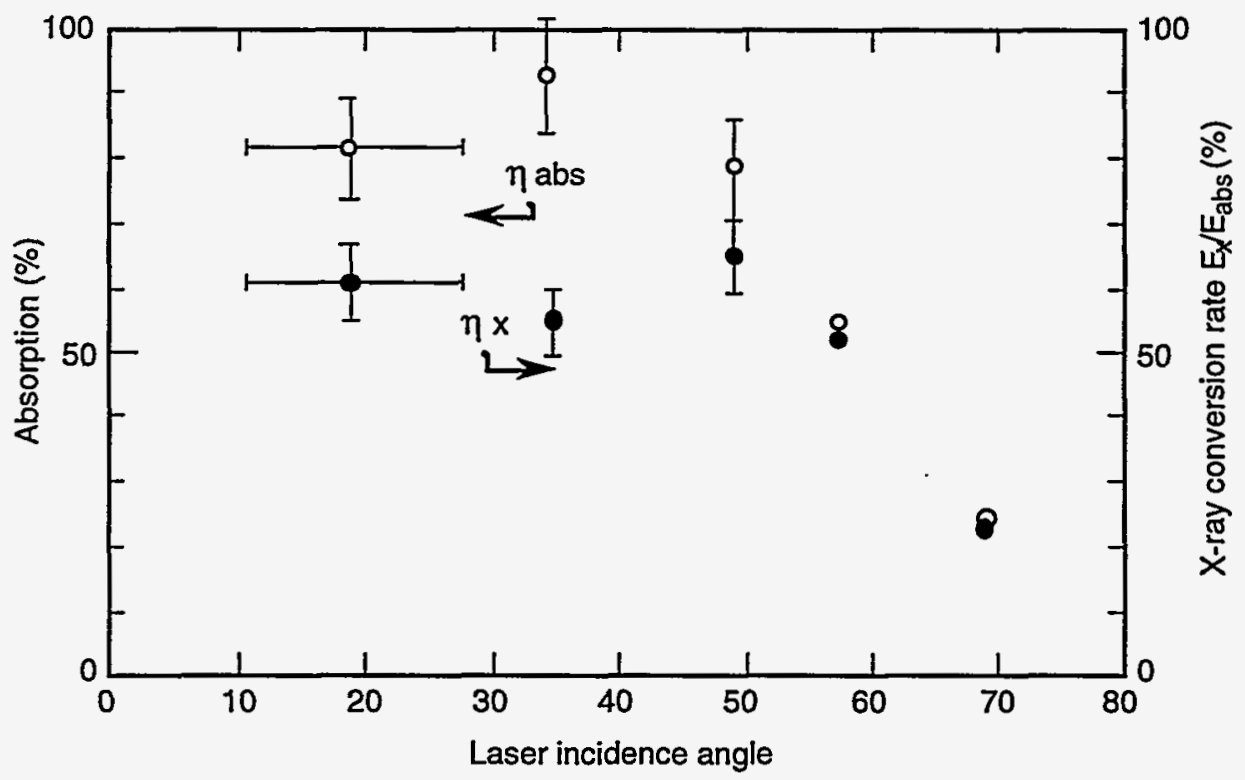

Figure VIII-9. Effect of angle of incidence of laser on $x$-ray conversion efficiency for intensity of $10^{14}$ $W / \mathrm{cm}^{2}$ on a solid gold target. The left-hand axis refers to the open circles, which measure absorption of the laser light. The right-hand axis refers to the filled circles and represents the $x$-ray conversion efficiency relative to the absorbed laser energy. 
the target, represented by the open circles. The right-hand axis refers to $x$-ray conversion efficiency measured relative to the absorbed energy, represented by the filled circles.

To convert this efficiency relative to absorbed energy to conversion efficiency relative to the incident energy, multiply the $E_{x} / E_{a b s}$ by the absorption percent. This gives, for example, for the $20^{\circ}$ result, a conversion efficiency of $46 \%$, which is in keeping with the $40 \%$ figure noted above. Efficiency does not drop off until the angle of incidence becomes greater than $45^{\circ}$. This alleviates experimental design constraints. The actual change in spectral shape as the angle is increased can also be shown not to deviate for angles less than $45^{\circ}$. These facts indicate the uniformity of the spectrum at angles less than $45^{\circ}$ and give an indication that angles up to as high as $50^{\circ}$ preserve a similar $x$-ray spectral character.

\section{Laser Intensity vs Conversion Efficiency}

The next important question concerns conversion efficiency from the laser light to $x$-rays as a function of intensity on target. In Fig. VIII-1 it can be seen that the conversion efficiency is roughly constant from $10^{12}$ to $10^{13} \mathrm{~W} / \mathrm{cm}^{2}$. However, the question arises: What is the behavior at higher or lower irradiance? There is data from the rear side of the target to indicate that the conversion stays roughly constant, at least to $10^{14} \mathrm{~W} / \mathrm{cm}^{2}$. Beyond this irradiance, the onset of laser-driven parametric processes will start to play a role and the benign nature of the x-ray conversion process is not guaranteed. Note that with the advent of smoothed beams this statement should be seen as worthy of investigation.

On the lower irradiance side, the broad definition of the soft x-ray region-from $50 \mathrm{eV}$ through $1250 \mathrm{eV}$-allows a wide range of temperatures over which the plasma will emit $x$-rays in this spectral band. Clearly the source will peak at lower energies and the total energy in the $x$-ray band will drop as laser intensity drops.

However, although we have no data on the conversion efficiency below $10^{12} \mathrm{~W} / \mathrm{cm}^{2}$, a reasonable estimate is to assume that:
- Absorption stays high at irradiances above $10^{9} \mathrm{~W} / \mathrm{cm}^{2}$, the threshold for plasma formation.

- The absorbed laser light will radiate as a blackbody radiator.

Therefore, we equate the laser intensity to the power radiated; i.e., the Stefan-Boltzmann relationship: $T_{e v} \sim\left(10^{-5} \mathrm{I}_{\text {laser }}\right)^{0.25}$. Here $\mathrm{T}_{\mathrm{ev}}$ is the plasma temperature in $\mathrm{eV}$ and $\mathrm{I}_{\text {laser }}$ is the laser intensity in $\mathrm{W} / \mathrm{cm}^{2}$. Thus, for an intensity of $10^{12}$ $\mathrm{W} / \mathrm{cm}^{2}$, the temperature of the plasma will be $\sim 18 \mathrm{eV}$, indicating that the peak of the emissionwere it a blackbody emitter-would be at $50 \mathrm{eV}$. The total energy emitted above $50 \mathrm{eV}$ would be half the laser energy. Since this is, in the spirit of the approximation, $40 \%$, this rough estimate of conversion efficiency can be extended down to intensities of $\sim 10^{10} \mathrm{~W} / \mathrm{cm}^{2}$, keeping in mind that there is not yet any data at these intensities.

\section{Hohlraum Radiation Temperature Scaling}

To illustrate various radiation environments and their scaling with hohlraum size, data taken at Nova are presented in Fig. VIII-10. Equivalent radiation temperatures vs time are shown for three different variations of the gold hohlraum, as well as two different laser-pulse durations. All experiments shown used a total energy of $18 \mathrm{~kJ}$. The hohlraums were all cylindrical, with laser entrance holes in the sides for the $500-\mu \mathrm{m}$ - and $1000-\mu \mathrm{m}$-length hohlraums, and through the ends in the 2800- $\mu \mathrm{m}$-length hohlraum.

As can be seen from the empirical scaling law (shown in Fig. III-3), both the area of the interior walls and the area of holes affect the radiation environment. The holes for the two longer (therefore larger) hohlraums are $500 \mu \mathrm{m}$ in diameter for the laser entrance holes and $800 \mu \mathrm{m}$ for the x-ray diode observation line of sight. For the smaller hohlraum, each laser entrance hole is $300 \mu \mathrm{m}$ and the line of sight is $400 \mu \mathrm{m}$.

The observations in Fig. VIII-10 were made with a 12-channel $x$-ray-filtered diode array that is time-resolved and absolutely calibrated. To keep the laser light from emerging from the line of sight and to assist in maintaining its unobstructed area, a $1500-\AA$ aluminum foil supported on each side by $5 \mu \mathrm{m}$ of plastic was 


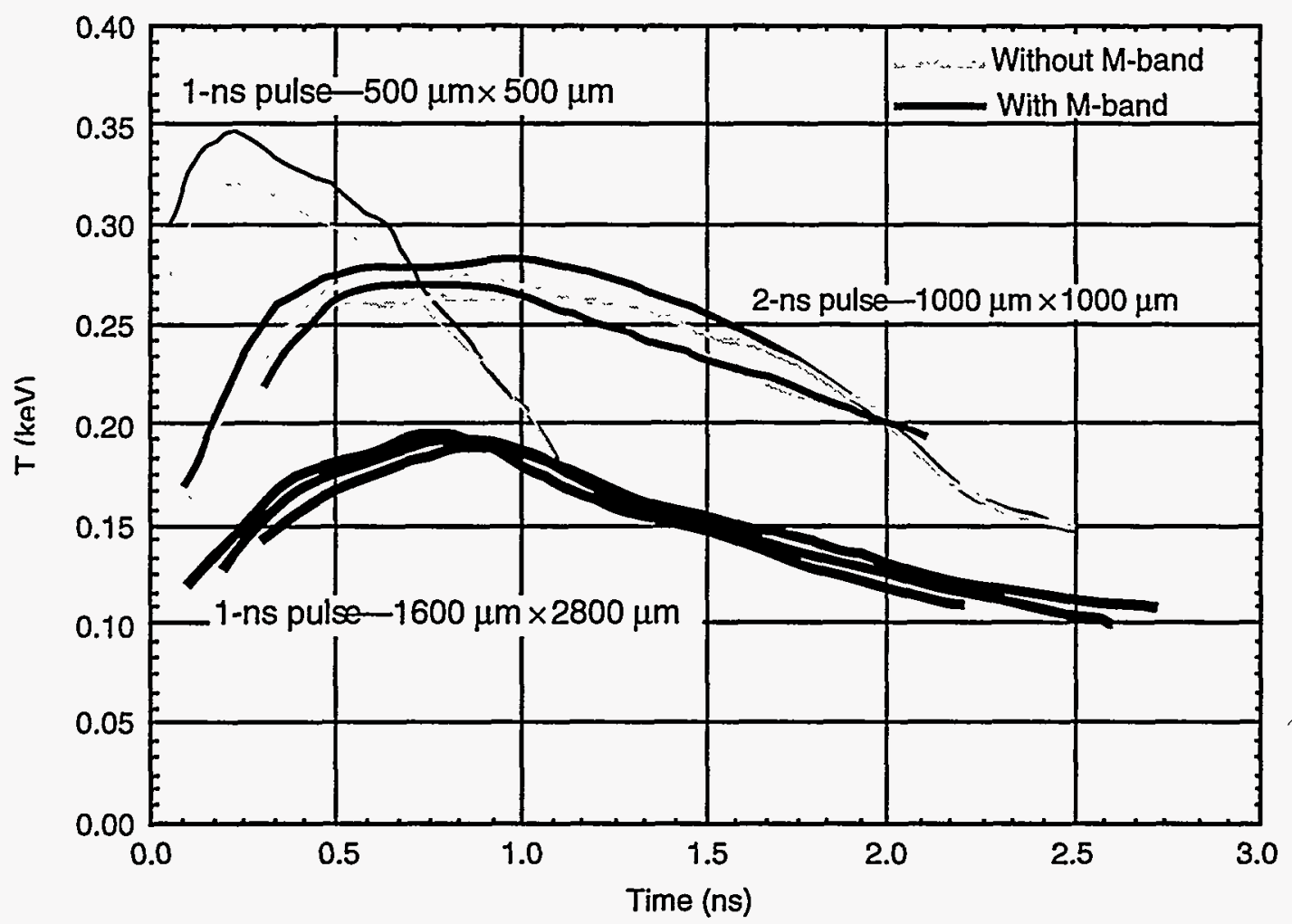

Figure VIII-10. Equivalent radiation temperature vs time for several gold hohlraums (schematics for the hohlraums are shown in Fig. III-2). The different curves for each hohlraum indicate the reproducibility of the data. The contribution of the gold $M$-band to radiation temperature is indicated by including data both with and without the M-band contribution.

inserted in the line of sight. The x-ray diode measures the specific intensity at the detector. The specific intensity is converted into an equivalent radiation temperature by integrating the source emissivity over all channels and equating this to the blackbody emissivity, given by $\sigma \mathrm{T}_{\mathrm{R}}{ }^{4}$. This is done for each of the times of interest. The contribution of the gold $M$-band, at $\sim 2-3 \mathrm{keV}$, is most pronounced in the small hohlraums.

To indicate the contributions of this highly non-Planckian part of the spectrum we also show in the figure the equivalent radiation temperature without the M-band contribution, indicated by the gray line. Note that the smaller the hohlraum, the higher the peak $\mathrm{T}_{\mathrm{R}}$ but the shorter the duration of the $\mathrm{x}$-ray flux. This is due to closure of the holes. Also, the relative $M$-band contribution to the $T_{R}$ is larger in the smaller hohlraums.
The $1000-\mu \mathrm{m}$ cylindrical hohlraum was used to investigate a longer duration pulse length. In this case, a 2-ns pulse was used, and the high peak $x$-ray flux is maintained for approximately $1 \mathrm{~ns}$, as can be seen in the figure.

The two different sets of curves for the $1000-\mu \mathrm{m}$ case (i. e., with and without M-bands) represent the effect of the angle of observation with respect to the laser-irradiated inner surface of the hohlraum. The two angles are $72^{\circ}$ and $0^{\circ}$ with respect to the cylinder axis. The curves with lower $T_{R}$ are for the viewing angle of $72^{\circ}$, and these show that when the detector observes less non-LTE gold emission in the hohlraum, it has a smaller M-band contribution as well as lower total flux. Thus, the radiation field in the hohlraum is a complicated function of viewing angle.

Finally, the figure shows three distinct experiments for the largest hohlraum, indicating 
the level of reproducibility of the x-ray production. These largest hohlraums are the standard configuration used in most inertial confinement fusion (ICF)-type experiments. In this case the M-band contribution is small because of the combined effects of the relatively large size of the hohlraum and the viewing angle, which does not allow a view of the laserirradiated spots in the hohlraum.

\section{B. Spectrally Narrow Sources}

The conversion of the laser radiation to discrete lines or narrow bands of lines represents a distinct field of study. The usual motivation for the study of conversion into narrow spectral lines has been the development of backlight (i.e., absorption) sources for use as probes of hot, dense matter. Because this has been the motivation, there is also an interest in the spatial extent of the source. In a manner similar to that used to estimate the conversion efficiency of the quasi-continuous source, it can be broadly assumed that it is possible to attain conversion efficiencies into a single spectral line of $0.1 \%$ to $1 \%$, with the lower efficiency being for higher $x$-ray energies than $4 \mathrm{keV}$, and the higher efficiencies being relevant for transition energies below $4 \mathrm{keV}$.

\section{Effect of $\mathbf{Z}$ on Emission}

The effect of $Z$ on the emission is that it changes the character of the emission from single spectral lines to bands. As shown in Fig. VIII-4, the gold $\mathrm{M}$-band is an example of the type of spectral structure that can expected. Conversion efficiency into these band-like structures is approximately $5 \%$ for $0.53-\mu \mathrm{m}$ light.

Figure VIII-11 shows a compilation of the results of experiments on several elements. ${ }^{7}$ The experiments irradiated a planar target of the material of interest with intensities of $10^{14}$ to $10^{15}$ $\mathrm{W} / \mathrm{cm}^{2}$. The dashed line indicates the results with $1.06 \mu \mathrm{m}$ light, while the solid line connects those points with $0.53 \mu \mathrm{m}$ light.

Although there are some differences in the performance of the targets under intensity variation, it is not significant in this broad outline of the results. Included in the figure are the results for aluminum silicon, titanium, nickel, and zinc $\mathrm{K}$-shell emission, and since this spans $\mathrm{Zs}$ of $13,14,22,28$, and 30 respectively, we have a fairly good indicator of levels of conversion.

Figure VIII-11 also shows conversion efficiency into the gold $(Z=69) \mathrm{M}$-bands. To change the conversion unit of this figure into fractional conversion in output joules, multiply the left-hand value $\xi_{x}$ by the $x$-ray line energy given on the bottom axis by $1.6 \times 10^{-16} \mathrm{~J} / \mathrm{keV}$. For example, the gold $\mathrm{M}$ bands are at an energy of $\sim 2.5 \mathrm{keV}$ and the $\xi_{\mathrm{x}}$ is $\sim 1.3 \times 10^{14}$, yielding $\sim 5 \%$ conversion.

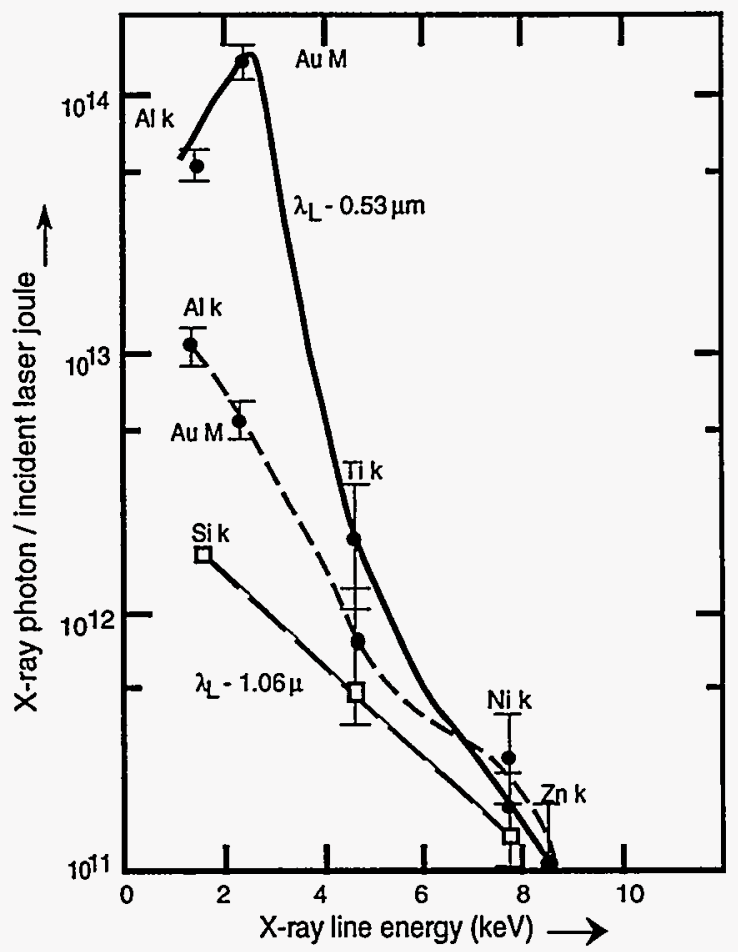

Figure VIII-11. Conversion efficiency of various discrete transitions, showing the K-shell lines of aluminum, silicon, titanium, nickel, and zinc as well as the $M$-band conversion of gold. Conversion efficiency is shown as a function of transition energy for intensities on the order of $\sim 10^{14} \mathrm{~W} / \mathrm{cm}^{2}$ on solid targets. The dashed lines tie together data for a 1.06- $\mu \mathrm{m}$ laser, and the solid line ties together the data for the $0.53-\mu \mathrm{m}$ laser. 


\section{Comments on Sparseness of Data}

In this discussion of spectrally narrow sources, we have given some simple rules for determining the conversion efficiency of laser light into $x$-rays. There are however, many gaps in the data, and particularly interesting is the lack of data from lower irradiances. For lower irradiances (that is, down to $10^{10} \mathrm{~W} / \mathrm{cm}^{2}$ ) the size of the source region can become large and the irradiation characteristics will be of interest. The levels of uniformity, angular dependence, temporal duration of the converted light, and spectral character are of interest.

Although this data does not exist, it is felt that the simple rules above can be used. Further work can, of course, be undertaken but this remains a very large field and it might be wise to have a first attempt at a design for the specific experiments of importance before seeking to obtain further quantitative data.

\section{X-ray Laser Sources}

There are three distinct paths of research for $x$-ray laser studies. First, there is research into the details of the mechanisms that have made the first viable laboratory $x$-ray laser possible. Second, there is an effort to use the currently developed lasers as a source to study other phenomena. Third, there is the continuing work to develop the $x$-ray laser as a light source for future applications. Although these three avenues are not completely distinct it is simpler to present the following discussions in this way.

\section{Studying X-ray Lasers}

Since the first demonstration of soft x-ray lasing using the collisional excitation mechanism in neon-like selenium, many other neon-like ions, ranging from copper $(Z=29)$ to silver $(Z=47)$, have been made to lase. ${ }^{8}$ However, attempts to produce lower- $Z$ neon-like $x$-ray lasers had been unsuccessful. In the effort to develop a tabletop $x$-ray laser that would require smaller highenergy laser drivers than Nova and could be used for applications such as biological imaging, nonlinear optics, holography, etc., a prepulse technique has been developed. This technique has been used successfully to produce lasing in many lower- $Z$ neon-like ions such as titanium $(Z=22)$, chromium $(Z=24)$, iron $(Z=26)$, and nitrogen $(Z=28) .{ }^{9}$ The use of this prepulse technique has opened up a new class of neon-like $\mathrm{x}$-ray lasers for investigation.

In studying the neon-like lasers it was noticed that the ions with odd $Z$ do not lase as well as those with even $Z$. Since elements with odd $Z$ have a nuclear spin and a nuclear moment while those with even $Z$ tend to have no nuclear spin, one possible explanation for this anomalous behavior is that hyperfine splitting is playing an important role in the gain of the neon-like laser lines for elements with odd $Z$.

To test this, we measured the line shape of the $J=0$ to $1,3 p-3 s$ transition in neon-like niobium and zirconium using a high-resolution grazing incidence grating spectrometer. We observed a $28-\mathrm{m} \AA$ splitting between the two largest hyperfine components in the niobium $(Z=41)$ line at $145.9 \AA$, in good agreement with theory. As predicted, no splitting was observed in zirconium $(Z=40) .{ }^{10}$

This $131-\mathrm{cm}^{-1}$ splitting is the largest hyperfine splitting ever observed and is also the shortest wavelength transition (145.9 $\AA$ ) and most highly ionized plasma ( 31 electrons) in which the hyperfine effect has been directly observed, with the exception of some very recent accelerator experiments in hydrogen-like bismuth done at GSI Darmstadt.

Further studies have been performed on the use of low-density foams as possible $x$-ray laser targets. Currently, $x$-ray laser experiments use either thin foils or thick slab targets at solid density. These targets are illuminated by Nova, causing them to heat and expand to reach the low densities required for lasing. This hydrodynamic expansion introduces large electron-density gradients in the plasma, which in turn result in large refraction effects as the $x$-rays propagate down the laser axis.

If targets could be fabricated from uniform low-density materials at the density required for lasing (i.e., $<3 \mathrm{mg} / \mathrm{cm}^{2}$ ), then beam propagation and ultimately laser coherence could be improved. Successful observations have been made of lasing in zirconium aerogel foam at 
$90 \mathrm{mg} / \mathrm{cm}^{3}$. At lower density, molybdenumdoped agar foam at $3 \mathrm{mg} / \mathrm{cm}^{3}$ and seleniumdoped agar at $8 \mathrm{mg} / \mathrm{cm}^{3}$ have been tried without success. However, the aerogel foams have much smaller cell sizes (500 \& compared with 1-2 $\mu \mathrm{m}$ for agar) and look very promising if the density can be lowered.

Very recent experiments are using a traveling-wave geometry to produce short-pulse $x$-ray lasers for use in imaging experiments on ICF targets. The traveling wave geometry enables us to drive the $x$-ray laser targets with short pulses ( $100 \mathrm{ps})$ while still using a long enough target $(-3 \mathrm{~cm}$ or greater) to produce strong laser output. We have recently observed a hundredfold contrast in neon-like germanium $x$-ray lasers observed with and against the direction of the traveling wave.

\section{Two-dimensional Plasma Imaging}

For two-dimensional plasma imaging, spatial resolution is currently limited by the pulse length of the x-ray laser, or alternatively by the gate time of the detector. Gated detectors with large active areas have temporal resolutions that are, at best, in the range of 100 to $200 \mathrm{ps}$. For characteristic expansion velocities of $10^{7} \mathrm{~cm} / \mathrm{s}$, this corresponds to a spatial resolution of 10-20 $\mu \mathrm{m}$, which is well below the resolution possible with available imaging optics. Therefore, there exists a real need to produce $x$-ray lasers with pulse durations shorter than the 200-ps FWHM routinely produced with exploding foil targets.

Demonstrated $x$-ray lasers are either recombination $x$-ray lasers, which rely on transient inversion to achieve gain, or collisionally pumped systems in which inversions are produced in steady-state conditions. The gain duration in conventional (or quasi-static) recombination $x$-ray lasers is determined by cooling and recombination rates, and typically results in pulse widths $>200 \mathrm{ps}$. This allows little control over the output pulse length without significantly reducing the total gain.

Collisionally pumped $x$-ray lasers have achieved large gain-length products and have been shown to operate over a wide range of pump conditions and a variety of targets. In addition, the wide-wavelength range over which collisionally pumped systems operate will make it possible to take advantage of, or alternatively avoid, line absorption in plasma-probing experiments.

To produce saturated $x$-ray lasers with pulse durations shorter than 20 ps and imaging resolutions of $\sim 2 \mu \mathrm{m}$, experiments have been performed that irradiate an exploding foil with multiple pulses. The concept is illustrated in Fig. VIII-12. The first pulse heats and explodes a thin foil target to produce a plasma with low density gradients. The second pulse then ionizes the preformed plasma to produce conditions suitable for high gain. The energy of the first pulse can be significantly lower than that of the second pulse, because high electron temperatures are not necessary. This improves the efficiency of this approach by reducing radiation and thermal losses during hydrodynamic expansion.

To test this technique and illustrate the importance of density gradients in $\mathrm{x}$-ray laser performance, experiments have been performed with yttrium $x$-ray laser targets. A key modification made to the laser beams was to introduce a tilt into the phase front of the beam. This allowed us to pump the foil in a traveling-wave configuration as shown in Figs. VIII-13 and DX-14. The traveling-wave configuration is important

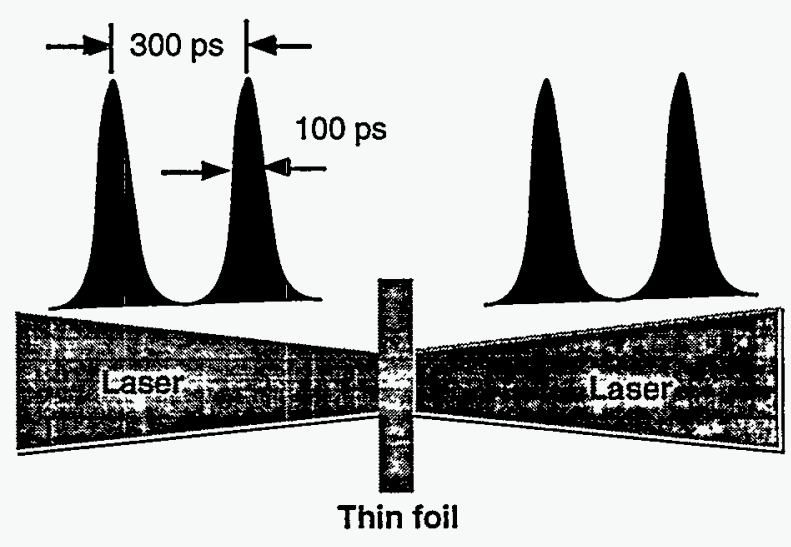

Figure VIII-12. Schematic of double-pulse irradiation technique for producing short-pulse $\mathrm{x}$-ray lasers. 


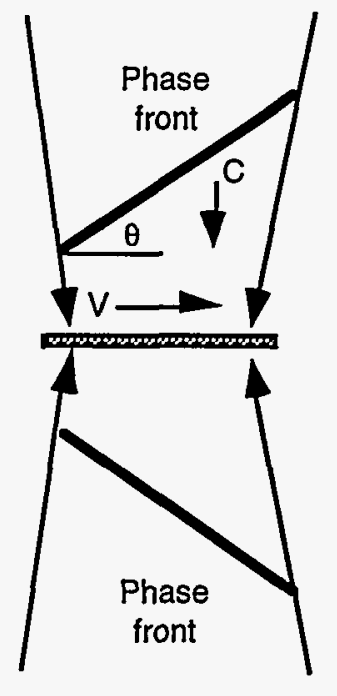

Figure VIII-13. Schematic of traveling-wave $x$ ray laser, illustrating how optical beam is tilted to generate traveling-wave pump along exploding foil.

given the short gain duration and finite propagation time of the $x$-ray laser across the foil (i.e., $100 \mathrm{ps}$ for $3-\mathrm{cm}$ foil). The traveling-wave pump along the exploding foil was generated by tilting an optical beam. The grating technique used to tilt the phase front of the traveling-wave beam is illustrated in Fig. VIII-14.

In the experiment, a 3-cm-long foil consisting of $2000 \AA$ of yttrium on $1000 \AA$ of plastic (lexan) was irradiated from both sides with two 100-ps pulses of $0.53-\mu \mathrm{m}$ laser light. The pulses were separated by $300 \mathrm{ps}$ and the foil was pumped using a traveling wave. The total intensity on the target was $2.4 \times 10^{14} \mathrm{~W} / \mathrm{cm}^{2}$ for the first pulse and $1.6 \times 10^{14} \mathrm{~W} / \mathrm{cm}^{2}$ for the second pulse. The measured soft $x$-ray spectrum in Fig. VIII-15 shows bright $\mathrm{J}=2-1,155-\AA$ x-ray line emission in $3 \mathrm{rd}$ and 4 th order of the grating. The effects of traveling wave pumping are clearly evident as the output is a factor of $\sim 200$ higher when the wave is traveling towards rather than away from the spectrometer. The two cases, wave traveling toward and wave traveling away from the spectrometer, are shown in Fig. VIII-14.

Figure VIII-16 shows the time-resolved emission spectra, in both hard $x$-ray and XUV range, of the traveling-wave experiment. The characteristic neon-like and fluorine-like 3-2 and

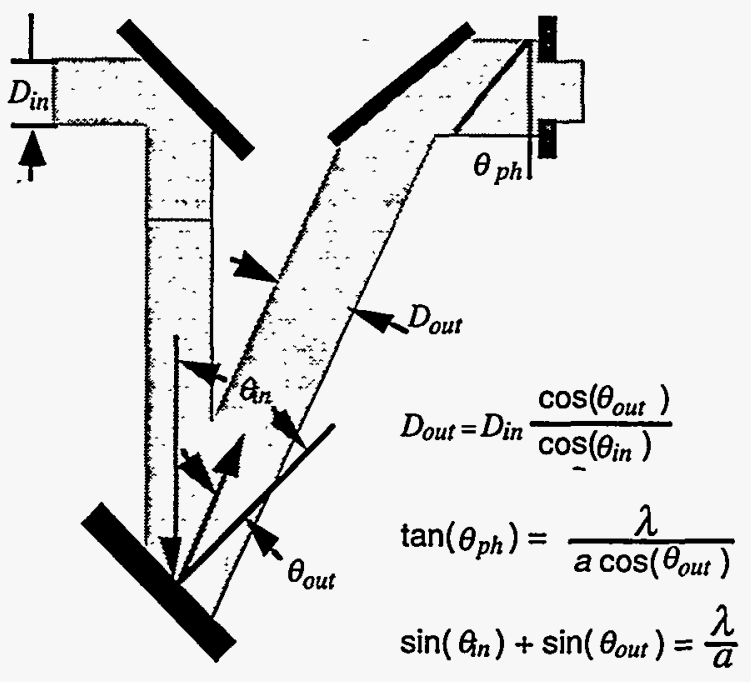

Figure VIII-14. Schematic of grating technique used to tilt phase front of traveling-wave $x$-ray laser beam to necessary angle.

hard $x$-ray emission spectrum (A) shows 4-2 transitions that indicate that appropriate ionization conditions for gain are produced during both pulses. In contrast, the $\mathrm{XUV}$ spectrum (B) shows strong $J=2-1 \mathrm{x}$-ray laser emission at $155 \AA$ during the second pulse only. The most likely reason for this is that during the first pulse the high density gradients refract the $x$-ray laser emission out of the gain region.

Figure VIII-17 shows the temporal evolution of both the x-ray laser and hard x-ray emission. The $x$-ray laser emission has a time duration of 45 ps FWHM, making this the shortest $x$-ray laser pulse width demonstrated.

Although in this experiment comparable intensities were used for both pulses, it would be clearly more efficient to reduce the intensity of the first pulse and increase the pulse separation to allow for expansion. Alternatively, a better pulse shape may consist of a low-intensity pedestal that irradiates the foil until the highintensity short-pulse irradiation begins. Using this approach we should be able achieve saturation $x$-ray laser output intensities of $4 \times 10^{11} \mathrm{~W} / \mathrm{cm}^{2}$ with less energy by a factor of $\sim 4$. Extending this technique to shorter output pulses will require shorter optical laser irradiation or a reduction in irradiation intensity to shorten the duration of neon-like ions. 

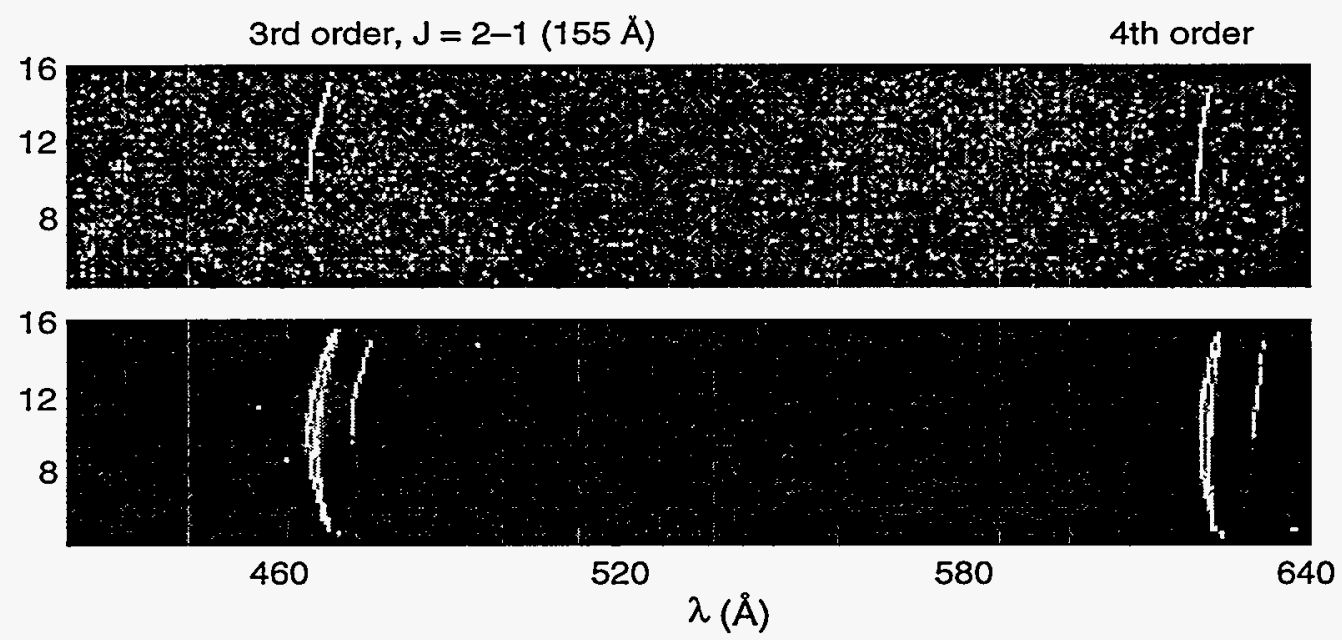

Figure VIII-15. $X$-ray line emission in 3rd and 4th order with traveling wave. Top shows the case where the beam is going away from detector and bottom shows where beam is going toward detector, where the output is increased by a factor of two.

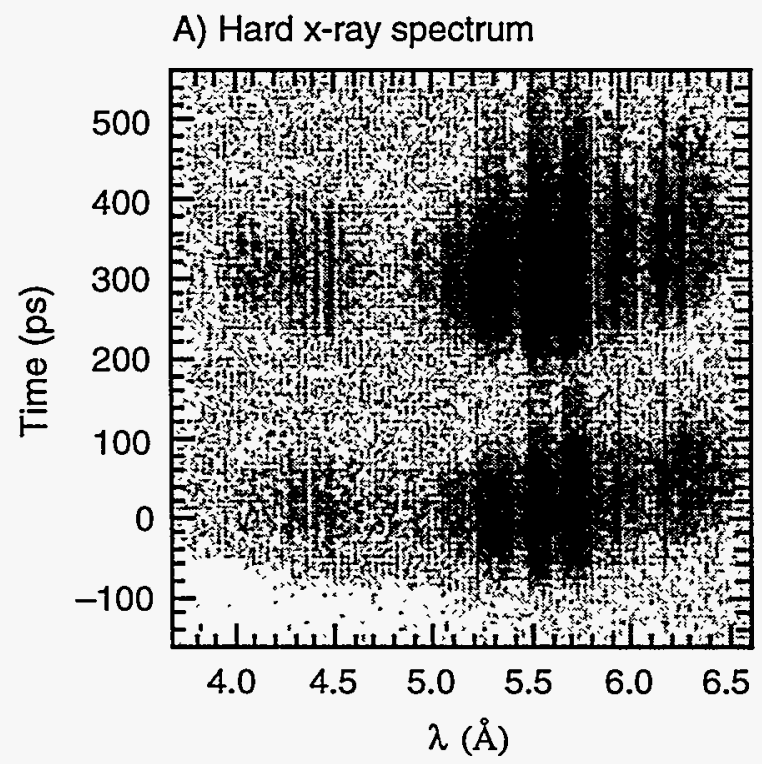

B) XUV spectrum

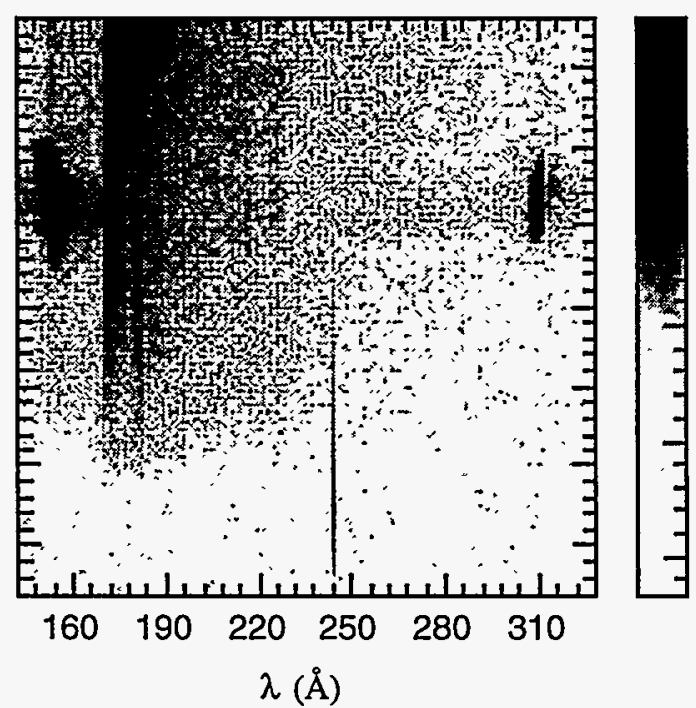

Figure VIII-16. Time history of hard $x$-ray and XUV emission spectra in neon-like yttrium. The hard $x$-ray emission spectrum (A) shows characteristic neon-like and fluorine-like 3-2 and 4-2 transitions that indicate that appropriate ionization conditions for gain are produced during both pulses. In contrast, the XUV spectrum (B) clearly shows strong $J=2-1 x$-ray laser emission at $155 \AA$ during the second pulse only. 


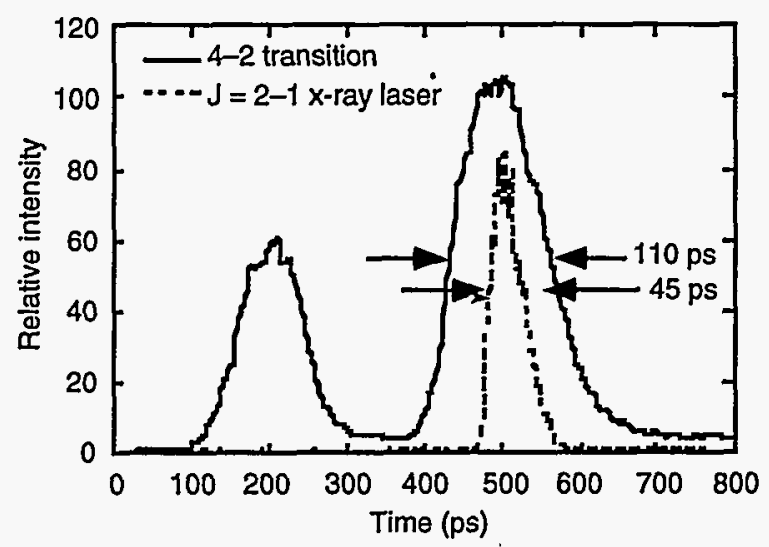

Figure VIII-17. Time history of hard x-ray (4-2 transitions) and $J=2-1 x$-ray laser transition in neon-like yttrium. The $x$-ray laser emission has a time duration of 45 ps FWHM.

\section{Development of $X$-ray Lasers as Probes}

Because the collisionally pumped soft $x$-ray lasers now operate over a wavelength range extending from 35 to $300 \AA$, and because these sources have high peak brightness, it is possible to begin using use them for $x$-ray imaging and plasma interferometry. The attraction of $x$-ray laser light sources is their extraordinary brightness, which was estimated to be equivalent to a Planckian of temperature of $40 \mathrm{keV} .11$

It is possible to demonstrate applications of the $x$-ray laser probe to long-scale-length plasmas using Moiré deflectometry and soft $x$-ray imaging. (Progress in the development of short-pulse $x$-ray lasers using a double-pulse irradiation technique that incorporates a traveling wave pump is described above.)

The first of the $x$-ray laser probe applications is diagrammed in Fig. VIII-18. Here a series of experiments has begun to characterize drive uniformity of thin-foil targets irradiated with laser beams that are smoothed with random phase plates. Targets of $10-\mu \mathrm{m}$ plastic foils overcoated with $3 \mu \mathrm{m}$ of aluminum are irradiated at $10^{14} \mathrm{~W} / \mathrm{cm}^{2}$ with a 1 -ns-square, $0.53-\mu \mathrm{m}$ wavelength beam from the Nova laser. These experiments image the accelerated foils by using the $\mathrm{x}$-ray laser as a backlight. In this experiment, $10-\mu \mathrm{m}$ plastic foils overcoated with $3 \mu \mathrm{m}$ of aluminum are irradiated at $10^{14} \mathrm{~W} / \mathrm{cm}^{2}$ with a

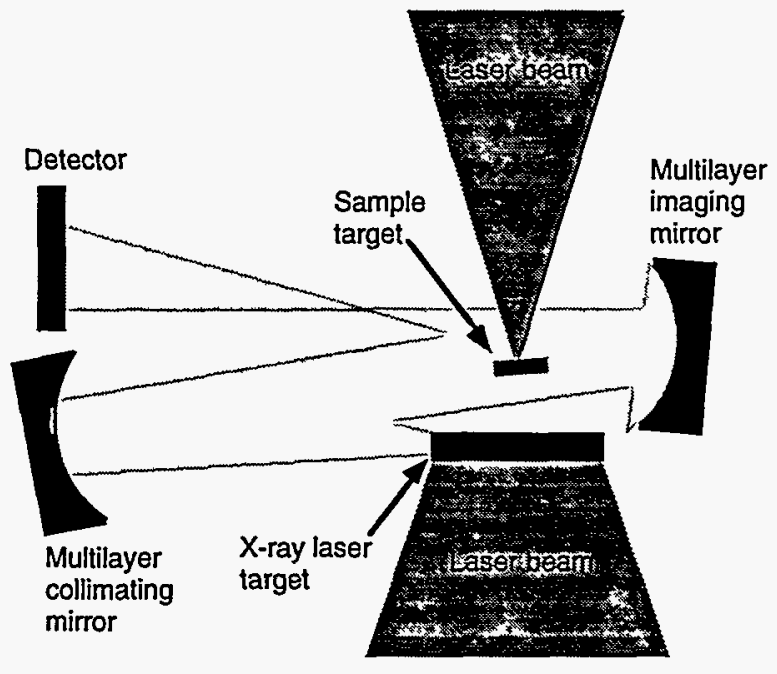

Figure VIII-18. Schematic of experimental setup for plasma imaging using the $x$-ray laser as a backlight. This setup is used to characterize drive uniformity of thin-foil targets. Targets of $10-\mu \mathrm{m}$ plastic foils overcoated with $3 \mu \mathrm{m}$ of aluminum are irradiated at $10^{14} \mathrm{~W} / \mathrm{cm}^{2}$ with a 1-ns-square, $0.53-\mu \mathrm{m}$-wavelength beam from the Nova laser. The accelerated foil is backlit with a 150-ps $x$-ray laser pulse from an exploded yttrium $x$-ray laser target.

1-ns-square, $0.53-\mu \mathrm{m}$-wavelength beam from the Nova laser. The beam's focal spot has an effectively flat-top distribution over a $700-\mu \mathrm{m}$ diameter region. The accelerated foil is backlit with a 150-ps x-ray laser pulse from an exploded yttrium $x$-ray laser target. The $x$-ray laser operates at $155 \AA$ and has an output energy of $\sim 7 \mathrm{~mJ}$.

In Fig. VIII-19 we show the x-ray laser backlit image from an accelerated foil experiment as recorded on an $x$-ray CCD detector. The foil is constructed of a $10-\mu \mathrm{m}$ plastic layer and a $3-\mu \mathrm{m}$ aluminum layer, irradiated, with the beam smoothed using random phase plates. The spatial resolution is estimated to be better than $2 \mu \mathrm{m}$. The 5- to 6- $\mu \mathrm{m}$ filament structure observed on the rear surface is likely due to the speckle pattern produced by the random phase plates.

This result clearly shows the merits of using $x$-ray lasers to image high-density plasmas. Although direct imaging using an $x$-ray laser does not require several of the attributes of the $x$-ray laser (specifically, small source size, narrow 


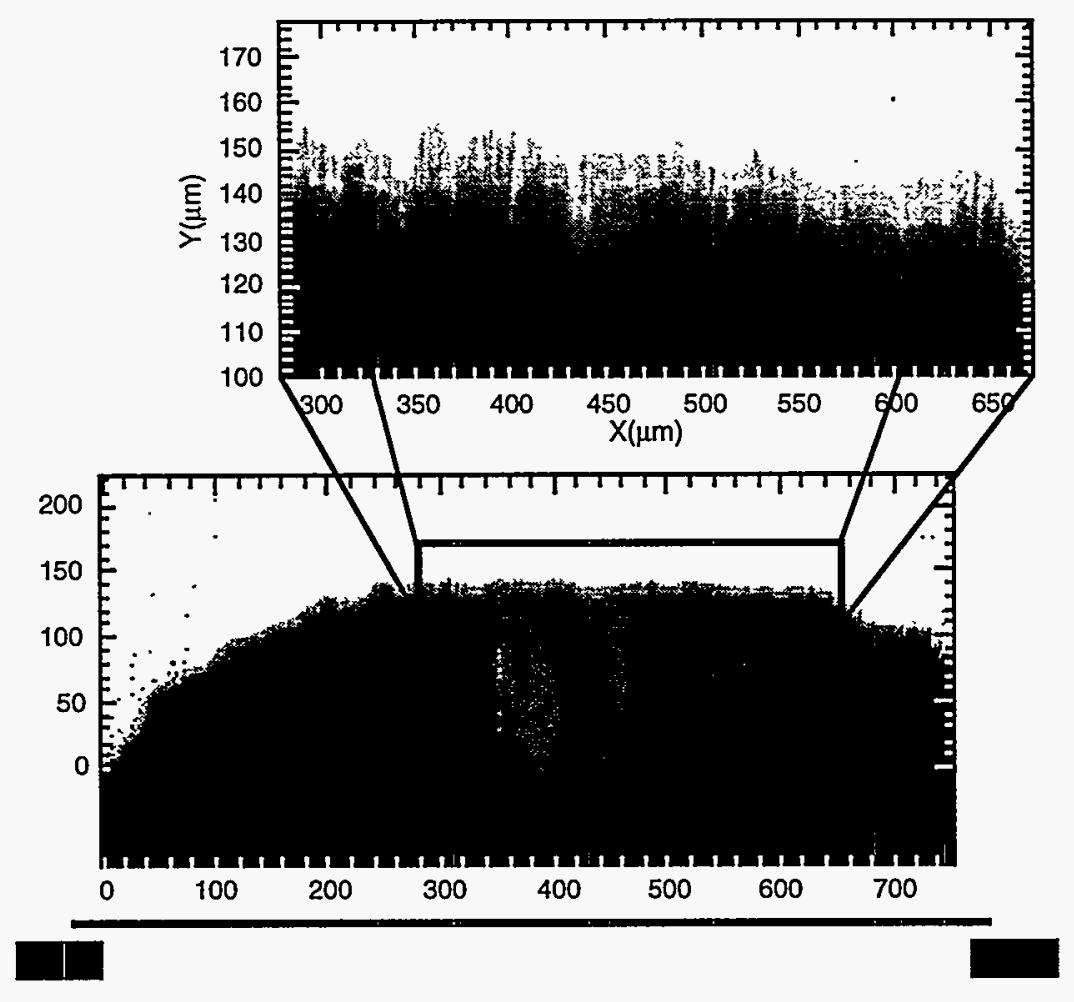

Laser intensity $10^{14} \mathrm{w} / \mathrm{cm}^{2}$

Figure VIII-19. X-ray-laser-backlit image of accelerated foil, recorded on an X-ray CCD detector. Foil consists of a 10- $\mu \mathrm{m}$ plastic layer and a $3-\mu \mathrm{m}$ aluminum layer, irradiated, with beam smoothed using random phase plates. Upper image is enlarged view of central region of foil, showing nonuniformities (filament structure) at the rear surface with dimensions characteristic of the speckle pattern produced by the random phase plates.

divergence, and narrow bandwidth), its high brightness makes it possible to neglect selfemission. Although not required for direct imaging, the short duration of the $x$-ray laser is useful in that it allows us to use an $x$-ray $C C D$ as the detector. The $x$-ray CCD has better spatial resolution $(27-\mu \mathrm{m}$ pixel) and higher dynamic range $(>1000)$ than existing gated microchannel plate systems.

Using a similar experimental setup, Moiré deflectometry experiments have been performed to measure electron density profile in a laserirradiated plastic plasma. The only difference between the imaging and Moiré experiments is the addition of two Ronchi rulings directly infront of the detector on the Moiré experiments. The rulings have a period of $10 \mu \mathrm{m}$ and a relative rotation of 4 degrees to give a fringe spacing of
$142 \mu \mathrm{m}$ in the image plane-i.e., $14.2 \mu \mathrm{m}$ in the object plane. The rulings were separated by $19.35 \mathrm{~mm}$ to give a sensitivity of $5.2 \mathrm{Mrad} /$ fringeshift at the object plane.

Figure VIII-20 shows the measured deflectogram obtained of a plastic target irradiated at $2.5 \times 10^{13} \mathrm{~W} / \mathrm{cm}^{2}$. The laser pulse was $1 \mathrm{~ns}$ square at a wavelength of $0.53 \mu \mathrm{m}$, and was focused to a 3-mm-diameter spot. The image shows the expected unperturbed Moiré pattern far away from the surface, but shows clear deflections closer to the irradiated side of the target. Analysis of the fringe pattern indicates peak electron densities of $2 \times 10^{21} \mathrm{~cm}^{-3}$ near the surface. This corresponds to a peak lineintegrated density of $6 \times 10^{20} \mathrm{~cm}^{-2}$, a factor of 30 higher than is accessible using conventional $U V$ interferometry at $\lambda \sim 2650 \AA$. 


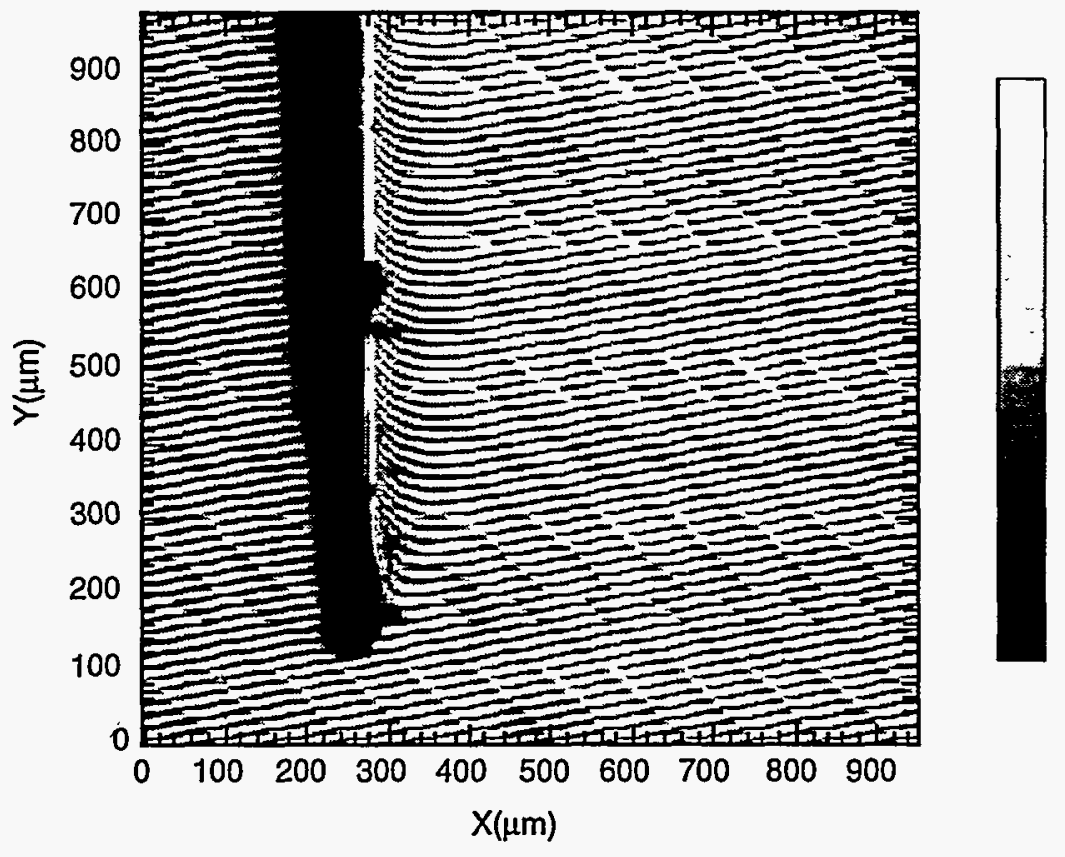

Figure VIII-20. Moiré deflectogram of laser-irradiated plastic target. Laser beam is incident from the right. The image shows the expected unperturbed Moiré pattern far away from the surface, but shows clear deflections closer to the irradiated side of the target. Analysis of the fringe pattern indicates peak electron densities of $2 \times 10^{21} \mathrm{~cm}^{-3}$ near the surface. This corresponds to a peak line-integrated density of $6 \times 10^{20} \mathrm{~cm}^{-2}$, a factor of 30 higher than is accessible using conventional UV interferometry at $\lambda \sim 2650 \AA$.

The second application employs the $x$-ray laser in an imaging microscope. Figure VIII-21 shows a schematic of the $x$-ray imaging microscope. It uses $x$-rays from a tantalum nickel-like collisionally pumped $x$-ray laser operating at $44.83 \AA$, which are collected and focused onto a specimen. The $x$-ray laser is generated by irradiating a $3.5-\mathrm{cm}$ long, $2000-\AA$ thick plastic foil coated with $900 \AA$ of tantalum using two cylindrically focused laser beams operating at a wavelength of $0.53 \mu \mathrm{m} .{ }^{12}$ The two beams heat the foil, which explodes to form a high-temperature plasma with low density gradients.

For this experiment, two optical beams from the Nova laser were used to generate a combined intensity on the target foil of $3.0 \times 10^{14} \mathrm{~W} / \mathrm{cm}^{2}$ for a duration of $500 \mathrm{ps}$. The $x$-ray laser pulse ( 200 ps FWHM) is characteristic of exploding foil amplifiers. The $x$-ray laser originates from $a$ $\sim 100-\mu \mathrm{m}$ diameter gain region at the center of the plasma and has a beam divergence of $10 \mathrm{Mrad}$ FWHM. The output energy is $\sim 10 \mu \mathrm{J}$, which gives a brightness of $10^{21}$ photons/(s- $\mathrm{Mrad}^{2} \mathrm{~mm}^{2}$ $0.01 \%$ BW)..$^{13}$

Figure VIII-22 shows an image of a resolution test pattern consisting of radial gold bars that taper down at the center to $\sim 350 \AA$. The gold bars are $1000 \AA$ thick and are on a $1000-\AA$ silicon nitride substrate. Features near the diffractionlimited resolution of $\sim 550 \AA$ are clearly observed. The nonuniform illumination pattern is due to the finite source size of the $x$-ray laser, which is demagnified onto the test pattern by the multilayer mirror collecting optic. (The effective source size of the $x$-ray laser is estimated to be $50 \mu \mathrm{m}$ by $80 \mu \mathrm{m}$.) The grainy nature of the image is due to the microchannel plate detector and the low signal levels.

Using this $\mathrm{x}$-ray microscope, images have been made of three rat sperm nuclei that have undergone different preparation techniques. The goal was to investigate the advantages of gold labeling and to access the effects of the various preparation techniques. 


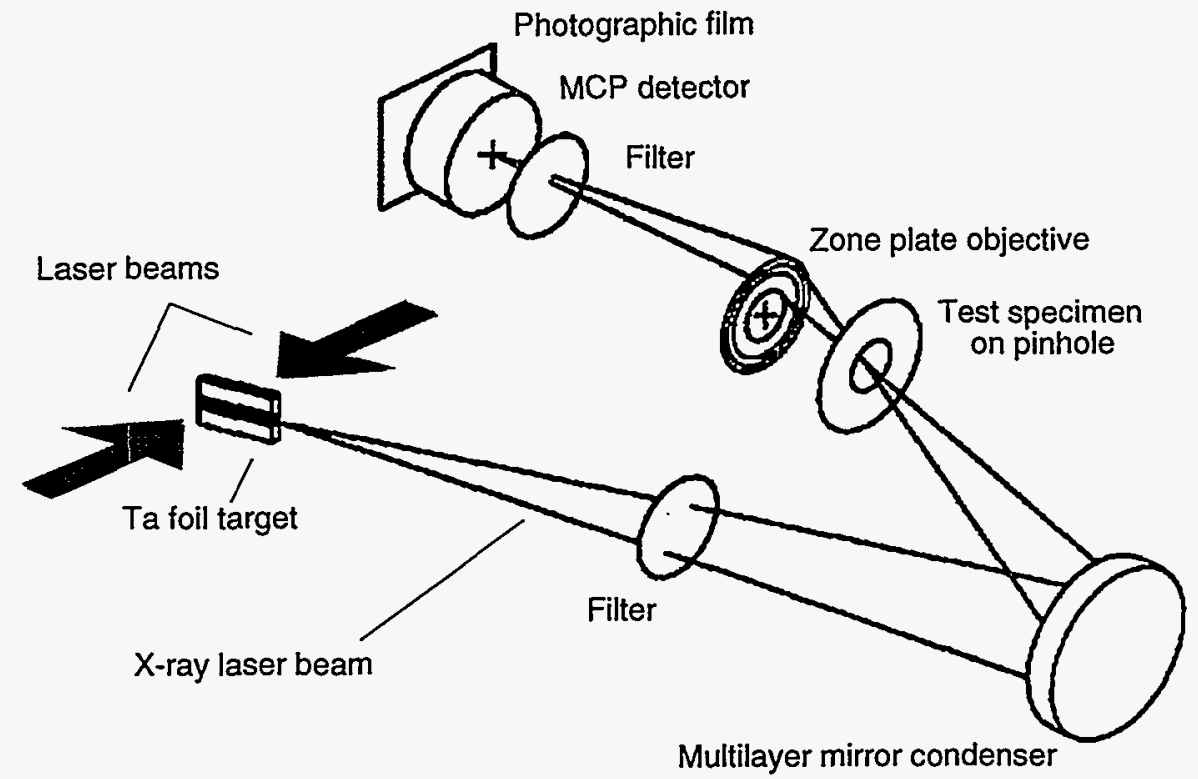

Figure VIII-21. Schematic of $x$-ray microscope, showing main components. The light source is a tantalum nickel-like collisionally pumped $x$-ray laser operating at $44.83 \AA$. The $x$-rays it produces are collected and focused onto a specimen using a spherical multilayer mirror.

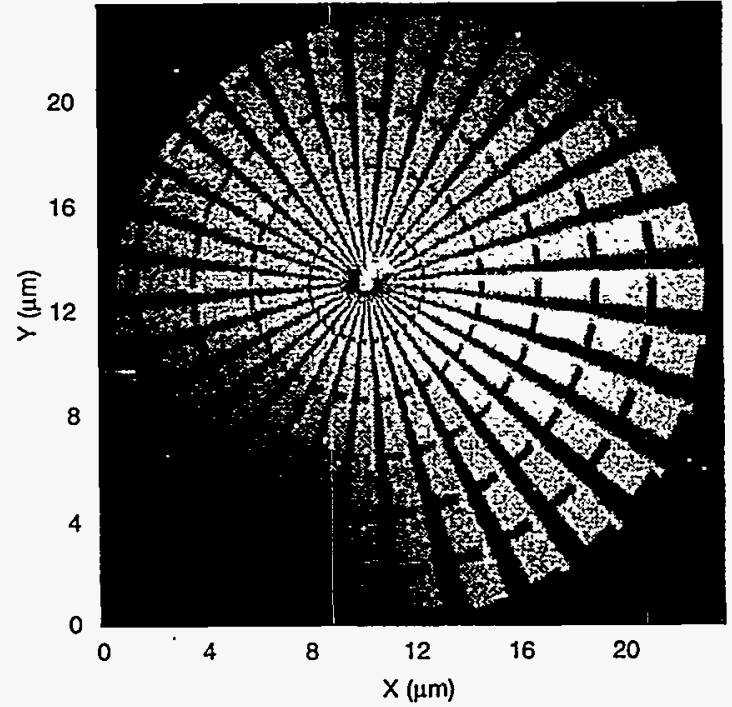

Figure VIII-22. Image of resolution test pattern obtained with $x$-ray laser microscope. The test pattern consists of 1000 - $\AA$-thick radial gold bars on a $1000-\dot{A}$ silicon nitride substrate. The bars taper down at the center to $\sim 350 \AA$. The nonuniform illumination pattern is due to the finite source size of the $x$-ray laser, which is demagnified onto the test pattern by the multilayer mirror collecting optic. The grainy nature of the image is due to the microchannel plate detector and the low signal levels.
The nuclei were first prepared by treating sperm isolated from rat epididymides to expose the DNA-protamine complex that comprises sperm chromatin. This was done by treating the sperm with a disulfide reducing agent and detergent to dissolve the tails, acrosome, and nuclear membranes. A droplet containing the amembraneous nuclei was deposited onto a silicon nitride window with dimensions $300 \times 300 \mu \mathrm{m}$ by $1000 \AA$ thick, and the liquid containing unbound nuclei was removed after 30 seconds.

Figure VIII-23 shows three $x$-ray microscope images of rat sperm nuclei. In (A) the rat sperm nucleus is unstained, in (B) the nucleus is "stained" with mouse antiprotamine 1 and tagged with $400-\AA$ diameter gold, and in (C) the nucleus is stained with antiprotamine 2 antibodies and goat anti-mouse antibodies tagged with $400-\AA$ diameter gold.

The images show distinct differences. The stained images show high concentrations of gold along the edge of the sperm and some evidence of individual $400-\AA$ gold particles on the surface. The clumping of the gold labels is particularly evident in the image of the nuclei stained with 


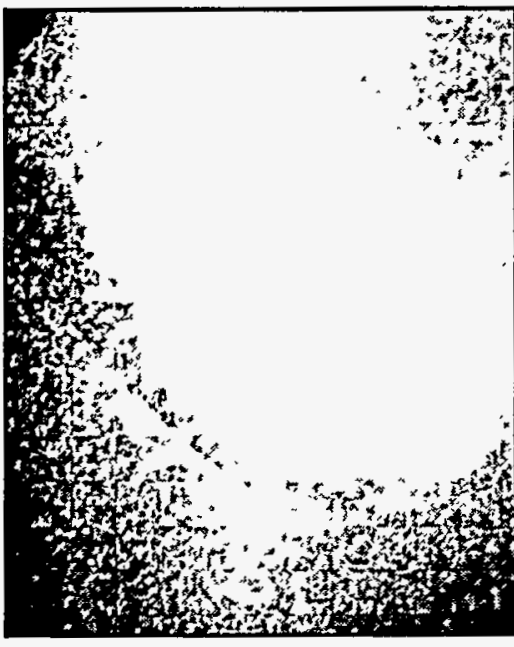

A)

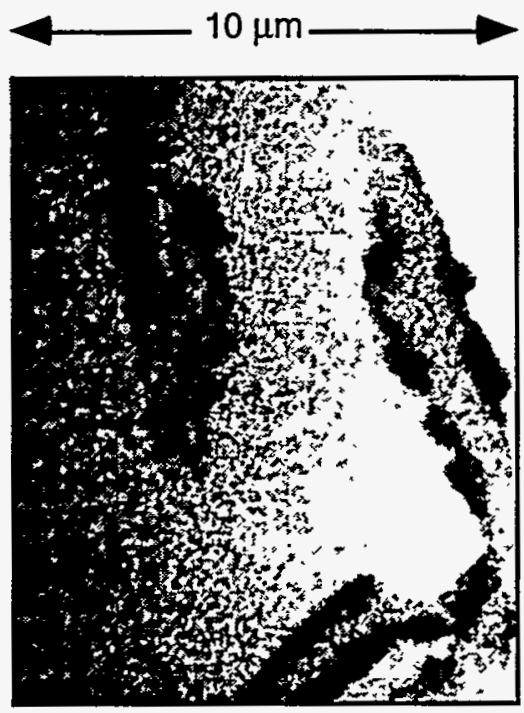

B)

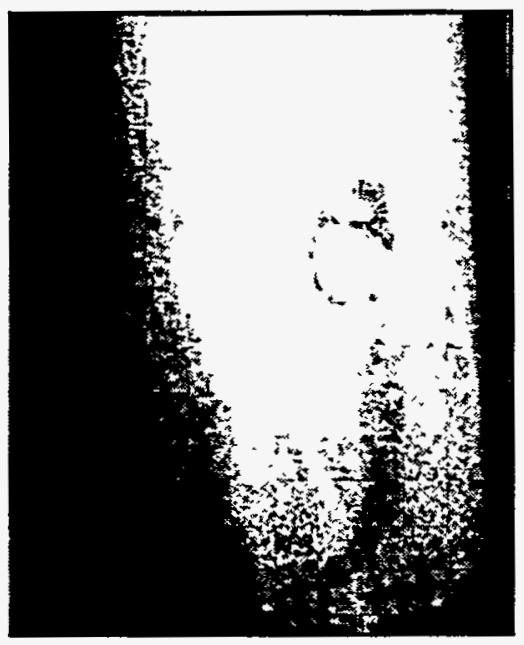

C)

Figure VIII-23. $X$-ray microscope images of rat sperm nuclei prepared using different techniques: A) unstained, with no gold labeling; B) stained with antiprotamine 1 and gold-labeled, and C) stained with antiprotamine 2 and gold-labeled. The stained images show high concentrations (clumping) of gold along the edge of the sperm and some evidence of individual $400-\AA$ gold particles on the surface. The clumping is particularly evident in (B). The frame structures observed in the antiprotamine-1stained nuclei (B) are a consequence of the preparation technique that unmasked internal sites for antibody binding. These images clearly show the value of gold labeling to enhance contrast, but also show the need for avoiding unnecessary preparation techniques that can lead to artifacts.

the antiprotamine- 1 antibody (B). The frame structures observed in the antiprotamine-1stained nuclei are a consequence of the preparation technique that unmasked internal sites for antibody binding. These images clearly show the value of gold labeling to enhance contrast, but also show the need for avoiding unnecessary preparation techniques that can lead to artifacts.

\section{XUV Interferometry}

Optical interferometry has an important history in the study and characterization of plasmas. However, probing of high-density and/or large plasmas has been difficult due to the high absorption of optical probes, the effects of refraction, and the impossibility of probing beyond critical densities. Recently, a neon-like yttrium $x$-ray laser has been used to develop the technology that will allow interferometry to be performed at wavelengths of $155 \AA$, which has a critical density $\sim 10^{25} \mathrm{~cm}^{-3}$.
In Fig. VIII-24, we show the experimental setup used to test a Mach-Zehnder interferometer suitable for probing high-density, long-scalelength plasmas. The output comes from a standard 3-cm-long yttrium $x$-ray laser with an output energy of $1 \mathrm{~mJ}$ in a 150-ps FWHM pulse. The output is collimated by a multilayer mirror and injected into a Mach-Zehnder interferometer. The interferometer uses molybdenum/silicon beamsplitters consisting of 8 layer pairs on $1000 \AA$ of silicon nitride. An imaging optic at the output of the interferometer images a plane within the interferometer where a secondary plasma is produced.

Figure.VIII-25 shows a recorded interferogram of a plasma formed by laser irradiation of a mylar target. Excellent fringe visibility is observed, clearly indicating the feasibility of this technique.

The limits of the current system are also apparent. In the figure we see that the blow-off of the plasma in the central region of the image is 


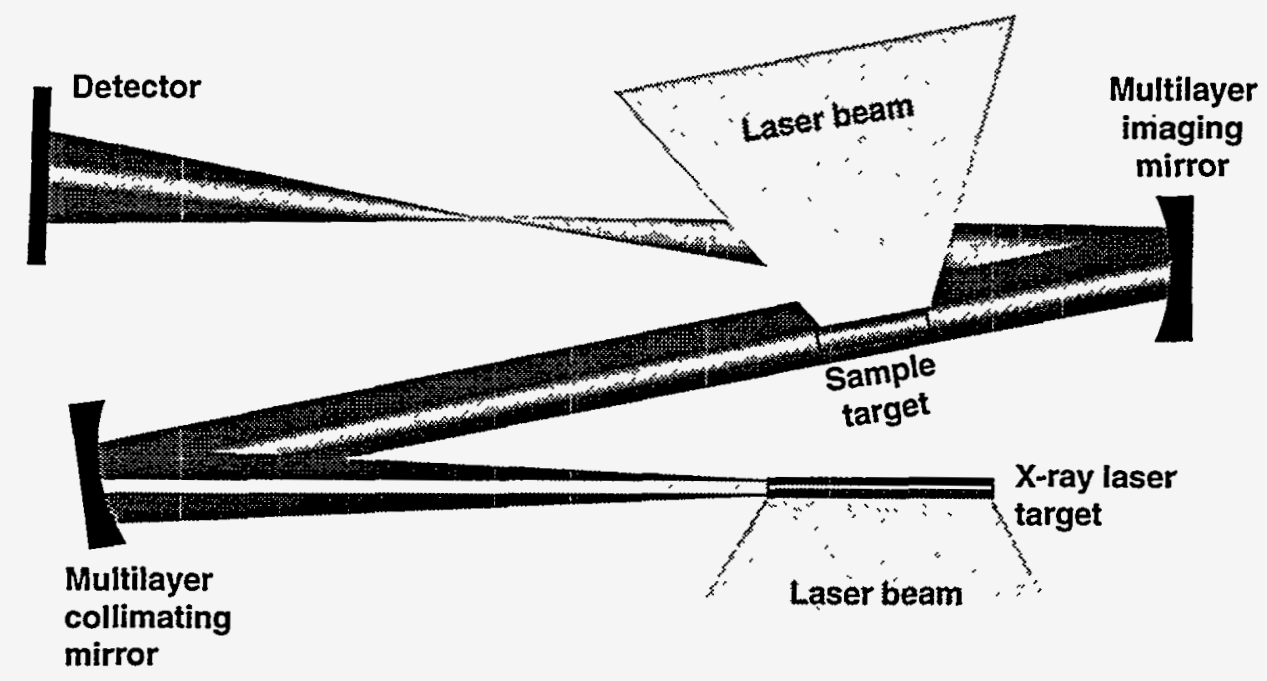

Figure VIII-24. Experimental setup for $x$-ray laser interferometry suitable for probing high-density, long-scale-length plasmas. The output comes from a standard 3-cm-long yttrium $x$-ray laser with an output energy of $1 \mathrm{~mJ}$ in a 150-ps FWHM pulse. The output is collimated by a multilayer mirror and injected into a Mach-Zehnder interferometer. The interferometer uses molybdenum/silicon beamsplitters consisting of 8 layer pairs on $1000 \AA$ of silicon nitride. An imaging optic at the output of the interferometer images a plane within the interferometer where a secondary plasma is produced.

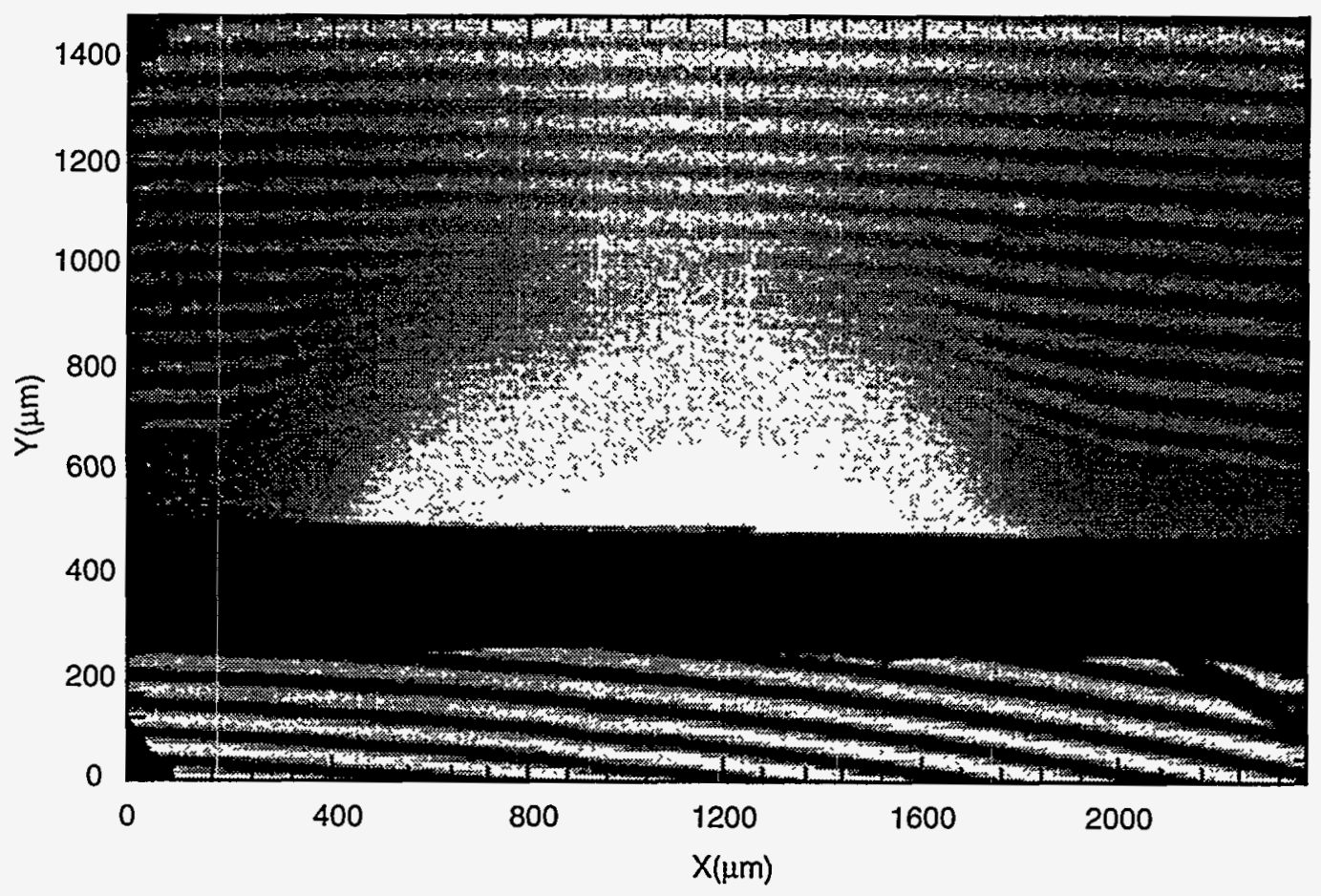

Figure VIII-25. Interferogram obtained using $x$-ray laser interferometer to image a plasma made by irradiating the surface of a mylar plastic with a second beam. The plasma-induced fringes can be readily seen, clearly indicating the feasibility of this technique. The thick solid band running horizontally is the plastic sample, while the bright spot above the plastic sample is the self-emission of the plastic. 
sufficient to completely obscure the laser. This is due to the existence of an oxygen transition near the laser wavelength at $155 \AA$, causing refraction. Further, the edges of the image show that the limit of the fringe count is caused by attenuation as the plasma becomes dense near the surface.

Thus, although the $x$-ray laser interferometer has been proven to work, there is still much work to be performed. This technology will be important in the characterization of NIF-scale plasmas. In addition, with the NIF we can push to shorter $x$-ray laser wavelengths, making this an even more important diagnostic tool.

\section{Future NIF Experiments}

Intense radiation at wavelengths extending from the far-infrared to the $x$-ray region of the spectrum can be generated by high-energy, lasermatter interaction. The characteristics of this radiation depend in detail on parameters such as the magnitude and mechanism of laser absorption, relevant atomic physics, the equation of state of the heated material, and hydrodynamics. In order to be able to tailor such radiation sources to the needs of specific experiments, considerable effort has gone into both detailed measurements of emitted spectra and extensive modeling work for a variety of materials and laser conditions. As a consequence of this work, scientists have developed sophisticated simulation tools that allow extrapolation of the capabilities that lie ahead using even higherenergy laser drivers, such as the NIF.

In the sections that follow we will discuss the types and capabilities of radiation sources possible using a high-energy laser driver, and discuss possible applications of these sources. First we discuss incoherent $x$-ray radiation sources; this includes both broadband sources and line emitters. Next we describe coherent sources; this includes an extrapolation of existing collisionally pumped $x$-ray lasers, new inner-shell pumped $x$-ray laser schemes, and high-order harmonic generation. The latter two of these coherent sources will take advantage of ultrashort-pulse capabilities on the NIF. Finally, we briefly discuss the potential of the NIF as a high-energy particle source.

\section{Incoherent X-ray Sources}

\section{Broadband Sources}

Laser-produced plasmas have long been recognized as efficient sources of $x$-rays. Nova experiments have already demonstrated conversion efficiencies as high as $50 \%$ from gold plasmas (see Fig. VIII-8 for the absolutely measured spectra of laser-irradiated targets of different elements, shown as a function of target Z). For the NIF this implies that megajoules of $x$-rays can be generated from a millimeter-size source. The effective blackbody temperature of this radiating plasma can be estimated from the Stefan-Boltzmann relationship to be given by $\mathrm{T}_{\mathrm{BB}}(\mathrm{eV}) \sim\left(10^{-5} \mathrm{I}_{\text {laser }}\right)^{0.25}$. Here $\mathrm{T}_{\mathrm{BB}}$ is the plasma temperature in $\mathrm{eV}$ and $\mathrm{I}_{\text {laser }}$ is the laser intensity in $W / \mathrm{cm}^{2}$.

Radiation sources driven on flat targets can be configured as point sources, with increased spatial coherence, or as large-area radiators for efficient radiative energy transfer in a closecoupled geometry. The most intense radiation environment created using a high-energy laser is found inside a radiation case, called a hohlraum.

In a hohlraum, laser energy is focused on the interior walls of a hollow cylinder that has laser entrance holes on the ends. The interior walls of the hohlraum are vaporized and heated to a high temperature. (This temperature has been measured at Nova; typical data for its temporal dependence is shown in Fig. VIII-10.) Section III, Experimental Capabilities, under "Hohlraum Radiation Sources," has information about the types of hohlraum radiation environments now available. With this information and a simple model (see Fig. III-3), simple extrapolations can be made to the NIF. At the NIF, by the simplest scaling, the radiation temperature in hohlraums will be increased by more than a factor of two, with energy outputs approaching two orders of magnitude larger than currently available.

Continuum $x$-ray sources, conveniently described by effective blackbody temperatures in regions of the spectrum determined by the specific ionic species contained in the plasma, can easily be spectrally filtered and tailored for a wide variety of applications, including optical pumping of absorbing targets in materials 
research and astrophysics. For examples refer to Section IV, Astrophysics and Space Physics; Section VI, Material Properties; and Section IX, Radiative Properties.

Laser-driven sources can be configured to efficiently yield hard $x$-rays, such as those used in imaging and opacity experiments. At the highest energies, implosion experiments on the NIF will achieve capsule temperatures of 5-10 keV, which will produce extremely bright bursts of continuum, hard $x$-rays.

Short-pulse capability on the NIF will allow the generation of intense, hard $x$-ray sources in the ultrashort temporal regime. This capability will allow the study of the dynamics of materials undergoing rapid phase transformations on the time scale of individual atomic motion.

Such studies in the past have been limited because of insufficient photon flux and because of the lack of hard $x$-rays. The hard $x$-rays are needed not only to heat but also to probe materials to sufficient depths to eliminate the effects caused by surface interactions and the effects of thermal wave propagation (shocks). (These effects are, of course, interesting in their own way-see Section VI, Material Properties). However, here we have the capability to study intrinsic material time scales. One intriguing possibility is the potential of rapidly heated materials to form metastable or short-lived phases; the type of pump-probe experiment possible with the NIF would allow observation of such material through characteristic $x$-ray scattering.

\section{Narrow or Line Sources}

Line sources can yield narrow-bandwidth radiation with increased temporal coherence. They represent an important diagnostic tool for hard $x$-ray imaging and opacity measurements. Conversion efficiency into a single spectral line has been measured to vary from $1 \%$ at $x$-ray energies below $3-4 \mathrm{keV}$ to $0.1 \%$ beyond $4 \mathrm{keV}$.

The character of the emission spectra is strongly affected by the dominant shell. For instance, $\mathrm{K}$-shell emitters are dominated by isolated strong hydrogen-like and helium-like line emission. In contrast, high- $Z$ emitters such as gold are dominated by large bands of unresolved transitions. Reference 1 contains a compilation of the experimental results for a variety of elements and laser conditions.

A NIF laser will allow higher ionization states to be accessed, extending the region of monochromatic hard $x$-ray sources to the hard $x$-ray region. The application of narrow bandwidth sources for the probing of polycrystalline structures is discussed in Section VI, Material Properties.

\section{Coherent X-ray Sources}

\section{X-ray Lasers}

Plasma-driven lasers now operate over a wavelength range extending from the ultraviolet down to the soft $x$-ray region near $35 \AA .{ }^{14}$ Such systems utilize single-pulse optical lasers to produce a hot and uniform plasma suitable for laser amplification and propagation. Figure VIII-26 shows a graph of both calculated and measured wavelengths of the dominant $x$-ray laser lines in collisionally pumped systems. Wavelengths are shown as a function of atomic number. With the NIF it would be straightforward to extend collisionally pumped neon-like $3 \mathrm{p}-3 \mathrm{~s}$ and nickel-like $4 d-4 p$ systems to short wavelengths and high output energies.

Previous Nova $x$-ray laser experiments have found that the total irradiance required in a 500 -ps pulse of $0.53-\mu \mathrm{m}$ laser irradiation scales with $x$-ray laser wavelength $\lambda$ is

$$
\mathrm{I}\left(\mathrm{W} / \mathrm{cm}^{2}\right) \sim 2 \times 10^{17}(\lambda / 20)^{-3.5}
$$

for neon-like systems and

$$
\mathrm{I}\left(\mathrm{W} / \mathrm{cm}^{2}\right) \sim 4.3 \times 10^{15}(\lambda / 20)^{-3.5}
$$

for nickel-like systems. Here $\lambda$ is the wavelength in Angstroms of the short wavelength J $=0-1$ in nickel-like and the brightest $\mathrm{J}=2-1$ line in neon-like systems.

Extending neon-like $x$-ray lasers below wavelengths of $20 \AA$ will be difficult given the high power requirements. Nevertheless, the high gain of these systems will make it possible to produce saturated $x$-ray lasers with plasma lengths of $2-3 \mathrm{~cm}$.

Extrapolating the nickel-like system to uranium with a J = 0-1 transition at $21.46 \AA$, we estimate that an irradiance of $4 \times 10^{15} \mathrm{~W} / \mathrm{cm}^{2}$ would be required to make an $x$-ray laser at this 


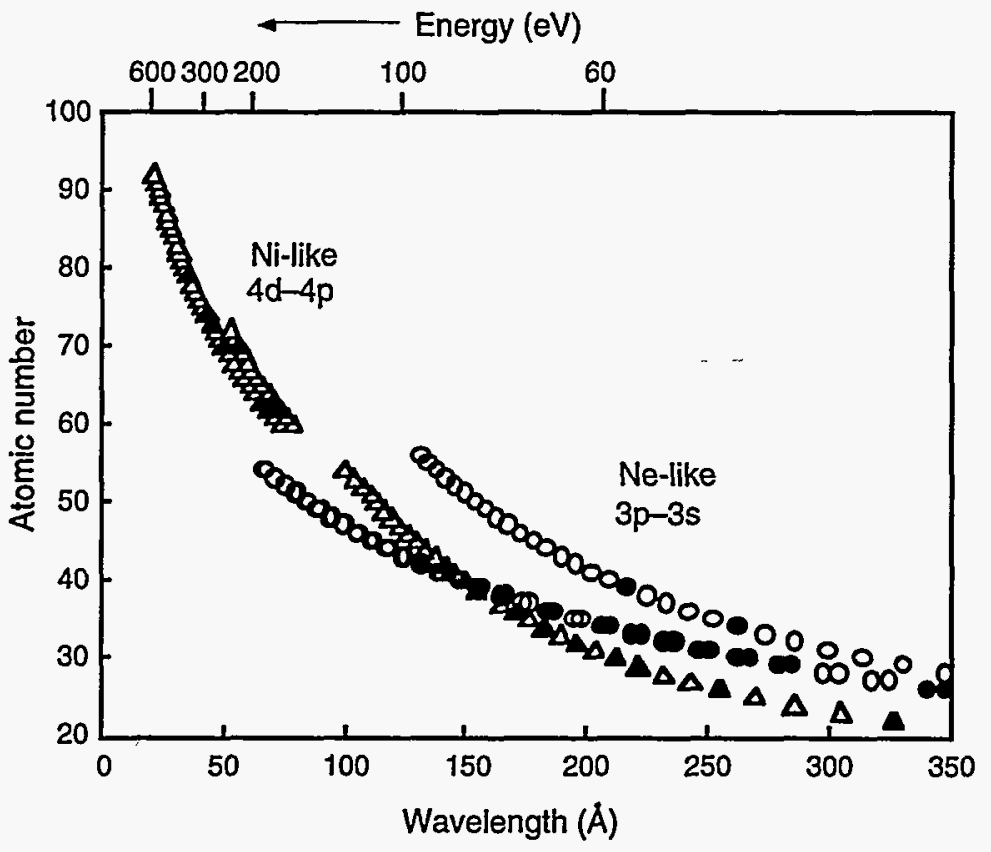

Figure VIII-26. Collisionally pumped $x$-ray laser wavelengths as a function of atomic number. The graph shows calculated wavelengths (open symbols) and measured wavelengths (solid symbols). The NIF would allow the probing of short wavelengths and high output energies.

wavelength. If we assume a 500- $\mu \mathrm{m}$-wide line focus, a 6-cm-long laser, and a 500-ps pulse, the energy required to saturate a nickel-like uranium laser is approximately $500 \mathrm{~kJ}$, well within the design limits of the NIF. The output $x$-ray laser energy from a saturated amplifier is calculated to be $\sim 0.2 \mathrm{~J}$. Using $\mathrm{x}$-ray optics to focus the $\mathrm{x}$-ray laser, we will be able to achieve peak intensities of $1 \times 10^{17} \mathrm{~W} / \mathrm{cm}^{2}$.

Numerous candidates have been proposed for photopumped $x$-ray lasers, yet to date no successful demonstration has been reported. The difficulty in these experiments remains producing bright $x$-ray pump sources that can efficiently couple to the candidate plasma. A NIFsize facility will allow large-volume pump plasmas to be produced that can efficiently pump candidate plasmas and allow testing of this promising concept.

The short-pulse capabilities of the NIF will allow us to extend $x$-ray lasers to the hard $x$-ray regime and to investigate a variety of new $x$-ray laser schemes, including inner-shell pumped and optical-field ionized lasers.
Inner-shell pumped $x$-ray lasers rely on bright $x$-ray sources to produce inner-shell vacancies and direct population inversion. The fast auger-decay rates of these systems make it necessary to use extremely short $x$-ray pulses. The main advantage of inner-shell pumped systems is the prospect of generating shortwavelength lasers with low- $Z$ atomic systems; for example, $10-\AA$ lasers from neon. Kapteyn, 15 and more recently Strobel et al., ${ }^{16}$ have investigated the feasibility of these systems and have shown that high gains are possible with bright $100-\mathrm{fs}^{\circ}$ $x$-ray pump sources.

Recombination $x$-ray lasers, which rely on fast three-body recombination in a cold plasma to produce an inversion, have long been viewed as an alternative to collisionally pumped systems, offering the potential for higher conversion efficiencies. To date, however, it has been difficult to achieve large gain length products (or alternatively, saturated output) in these systems. A primary reason has been the difficulty of producing long uniform plasmas suitable for 
inversion and $x$-ray propagation. A NIF-size facility will allow recombination systems to be adequately tested because of the large-volume plasmas available.

Optical-field ionized $x$-ray lasers, which are a variant of standard collisional and/or recombination lasers, rely on a short laser pulse to multiphoton-ionize a neutral gas. The short optical pulse reduces collisional heating - or it can, by design, enhance it. This $\mathrm{x}$-ray laser scheme has the potential for a short output pulse, on the order of $100 \mathrm{fs}$.

\section{High-Order Harmonic Generation}

The generation of odd harmonics, up to the eleventh, from the interaction of high-intensity lasers with dense gases had long been observed..$^{17}$ However, researchers have now measured harmonics extending to the 109th of $811-\mathrm{nm}$ radiation and the $143 \mathrm{rd}$ of $1053-\mathrm{nm}$ radiation, by shortening pulse duration to avoid ionization and by increasing intensity. ${ }^{18,19}$

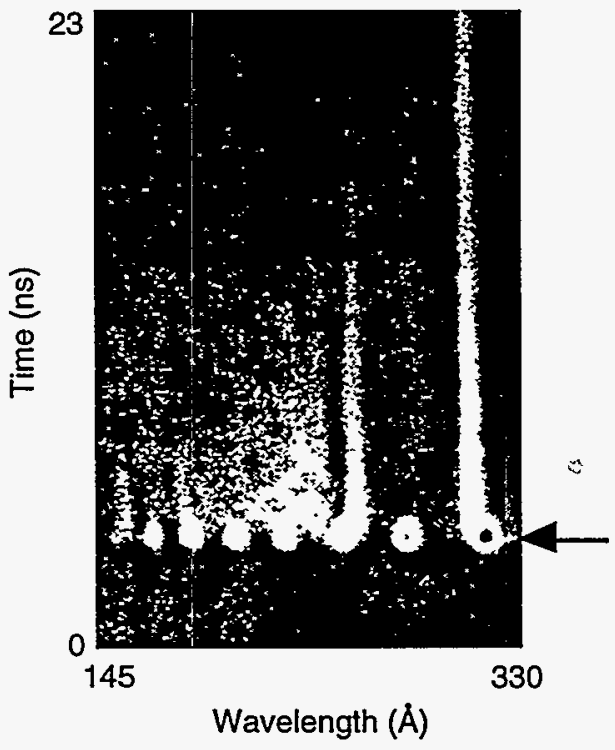

The harmonics in these experiments result from the periodic oscillation of quasi-free electrons across the atomic potential. As the electron passes the nucleus, it suffers an impulsive distortion in its trajectory, which generates a broad spectrum of radiation. Because the harmonics are produced only by the driving field of the laser, their pulse width is equivalent to that of the driving optical pulse.

Figure VIII-27 shows an example of the type of data obtained from harmonic generation experiments. In these experiments, high-order harmonics are used to generate coherent XUV radiation. In rare gases this can produce a radiation source that could be useful in the $200-\AA$ region. The experiment is quite straightforward, as the laser is focused into a gas cell containing, for example, helium. The laser is an $800-\mathrm{fs}-$ duration, 1.053- $\mu \mathrm{m}$-wavelength system with up to $8 \mathrm{~J}$. The diagnostic is a streak camera coupled to a XUV grating spectrometer that produces a flat field.

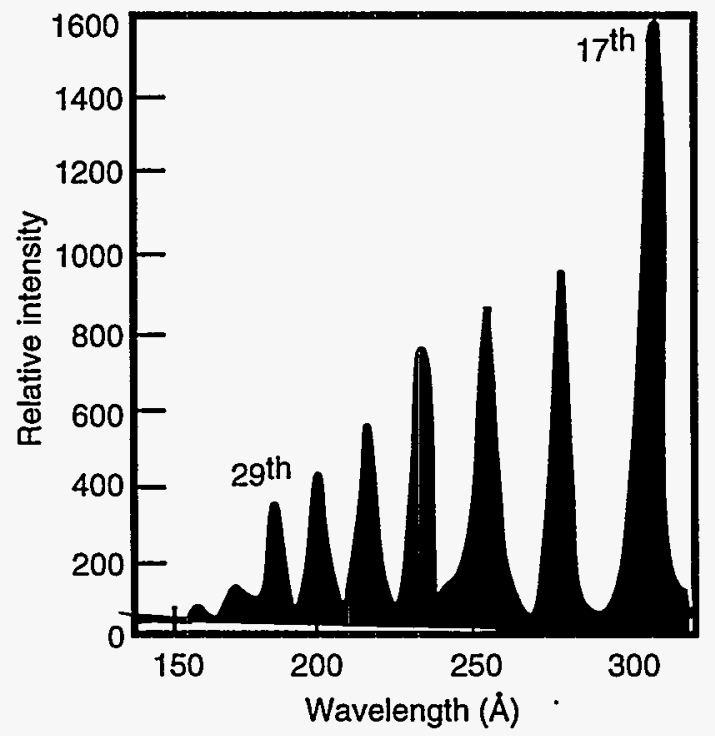

Figure VIII-27. Streak image of coherent XUV radiation generated by high-order harmonics. The streak image covers from 145 to $330 \AA$ in the abscissa and from 0 to 23 ns in the ordinate. The longwavelength, long-lived transitions are the Lyman series of hydrogen-like helium. The short-lived features that are evenly spaced in wavelength are the odd harmonics of the laser. On the right is an intensity trace through the harmonics taken at a time indicated by the arrow on the streak image. The trace indicates the existence of the 17th through the 31st harmonic, occurring at $170 \dot{A}$. The use of long focal-length lenses will make it possible to use the NIF to generate coherent tunable XUV radiation. 
On the left of the figure is a typical streak image, covering from 145 to $330 \AA$ in the abscissa and from 0 to $23 \mathrm{~ns}$ in the ordinate. The longwavelength, long-lived transitions are the Lyman series of hydrogen-like helium. On the right is an intensity trace through the harmonics that indicates the existence of the 17th through the 31st harmonic, occurring at $170 \AA$.

Coherence and overall conversion efficiency are limited by phase matching between the harmonic field and the incident laser field. Recent experiments have produced $\mathrm{XUV}$ harmonic radiation near $20 \mathrm{~nm}$ with conversion efficiencies as high as $10^{-7} .20$ Longer wavelength radiation, greater than $50 \mathrm{~nm}$, can be produced with a conversion efficiency exceeding $10^{-6}$. The use of long focal-length lenses will make it possible to use this technique on the NIF to generate microjoules of coherent tunable $\mathrm{XUV}$ radiation.

\section{Applications of X-ray Lasers}

Biological Imaging. The high brightness of $x$-ray lasers makes them well-suited for a variety of applications ranging from biological imaging and microscopy to nonlinear optics and

interferometry. $X$-ray microscopy offers a way to study structure in wet, thick, $2-10-\mu \mathrm{m}$, biological specimens. Demonstrated resolution is approximately five times better than that of conventional and confocal optical microscopes for samples that are thicker than the electron microscopes' limit of $0.4 \mu \mathrm{m} .{ }^{21} \mathrm{X}$-ray microscopy, then, allows whole cells or organelles to be studied without sectioning.

When dealing with wet specimens, the radiation dose imparted to the specimen by electron microscopy leads to mass loss and decomposition of specimens. Resolution is, therefore, limited by radiation damage and exposure time.

X-ray lasers, by virtue of their high brightness, have the potential for producing high-resolution biological images before significant damage occurs to the specimen. For instance, the tantalum nickel-like $x$-ray laser has been used to image dry biological gold-stained specimens. However, developing full threedimensional imaging of biological specimens will require a significant increase in the output energy of the tantalum x-ray laser. A NIF-size facility will make high-contrast imaging possible, by allowing us to produce saturated $x$-ray laser output at laser wavelengths above and below the carbon absorption edge at $44 \AA$.

Plasma Diagnostics. $X$-ray lasers are currently finding important applications as diagnostics tools for high-density plasmas. To date most of this work has used the neon-like yttrium x-ray laser operating at $155 \AA$, because of its high output power of $30 \mathrm{MW} .{ }^{22} X$-ray laser diagnostic techniques have significant advantages over conventional optical diagnostic techniques because they reduce refraction effects and eliminate problems with critical density layers. However, the high absorption of relevant plasmas at $155 \AA$ creates a real need to push the work on $x$-ray laser diagnostics to shorter wavelengths in order to allow accurate density measurements of cool plasmas at solid densities.

Nonlinear optics at soft $x$-ray wavelengths is an area largely ignored because of the difficulty in generating sufficient $x$-rays to make measurement viable. Numerous experiments have demonstrated multiphoton processes in the near UV by focusing optical lasers through neutral gases. To extend these experiments to the soft $x$-ray region, a plasma is necessary both to reduce absorption and to allow for resonant enhancement of the nonlinear susceptibility. One possibility that is particularly interesting is fourwave mixing, in which an optical laser is mixed with an $x$-ray laser to produce a tunable soft x-ray source. Aside from its clear applications, these experiments would allow measurements of nonlinear susceptibility to be made in a regime that is largely unexplored.

\section{Particle Sources}

A NIF laser will permit the generation of a wide variety of particle sources ranging from high-energy electrons and ions to neutrons and other fusion products. The generation of highenergy electrons through parametric instabilities in plasmas is discussed in Section VII, Plasma Physics. Pair production using high-intensity lasers has long been proposed but is outside the realm of existing facilities. The demonstration of 
pair production will be marginally possible with a NIF-size facility, as discussed in Section IV, Astrophysics and Space Physics.

At a high-energy facility like the NIF, implosion and subsequent burn will produce a variety of fusion products. Initial calculations indicate that neutron production from implosion and burn could exceed $10^{19}$ in a short, 100-ps burst. The use of these neutrons for producing uniform high-density plasmas exists, but this application is seriously limited by the low temperatures of current facilities. The high flux levels of higher temperatures on the NIF can be useful, however, for studying material properties in a regime where the generation and growth of dislocations become nonlinear.

\section{E. References}

1. K. Eidmann, Phys. Rev. A 41, 3270 (1990).

2. D. Kania, H. Kornblum, B. Hammel, J. Seely, C. Brown, U. Feldman, L. Da Silva, B. MacGowan, D. Montgomery, C. Back, R. Doyas, J. Edwards and R. Lee, Phys. Rev. A 46, 7853 (1992).

3. D. Matthews, E. Campbell, N. Ceglio, G. Hermes, R. Kauffman, L. Koppel, R. Lee, K. Manes, V. Rupert, V. Slivinsky, R. Turner, F. Ze, J. Appl. Phys. 54, 4260 (1983).

4. P. Carroll and G. O'Sullivan, Phys. Rev. A 25, 275 (1982).

5. K. Eidmann and T. Kishimoto, Appl. Phys. Lett. 49, 377 (1986).

6. H. Nishimura, H. Takabe, K. Kondo, T. Endo, H. Shiraga, K. Sugimoto, T. Nishikawa, Y. Kato, and S. Nakai, Phys. Rev. A 43, 3073 (1991).

7. D. Matthews, E. Campbell, N. Ceglio, G. Hermes, R. Kauffman, L. Koppel, R. Lee,
K. Manes, V. Rupert, V. Slivinsky, R. Turner, F. Ze, J. Appl. Phys. 54, 4260 (1983).

8. J. N. Nilsen et al., Phys. B 26, L243 (1993).

9. J. N. Nilsen et al., Phys. Rev. A 48, 4682 (1993).

10. J. N. Nilsen et al., Phys. Rev. Lett. 70, 3713 (1993).

11. D. L. Matthews et al., Phys. Rev. Lett. 45,110 (1985).

12. B. J. MacGowan et al., Phys. Rev. Lett. 65, 420 (1990).

13. L. Da Silva et al., Science 258, 269 (1992).

14. B. J. MacGowan et al., Phys. Fluids 4, 2326 (1992).

15. H. Kapteyn, Appl. Opt. 31, 4931 (1992).

16. G. L. Strobel, D. C. Eder, R. A. London, M. D. Rosen, R. W. Falcone, and S. P. Gordon, in "Inner-shell photo-ionized $x$-ray laser schemes" (SPIE Proceedings, Los Angeles, 1993), Vol. 1860, pp. 157.

17. J. F. Reintjes, Nonlinear Optical Parametric Processes in Liquids and Gases (Academic Press, Orlando, Fl, 1984).

18. J. J. Macklin, J. D. Kmetec, C. L. Gordon, Phys. Rev. Lett. 70, 766 (1993).

19. J. K. Crane, H. Nguyen, M. D. Perry (submitted for publication).

20. S. C. Wilks, W. L. Kruer, M. Tabak, and A. B. Langdon, Phys. Rev. Lett. 69, 1383 (1992).

21. L. B. Da Silva, J. E. Trebes, R. Balhorn, S. Mrowka, E. Anderson, D. T. Attwood, T. W. Barbee, J. Brase, M. Corzett, J. Gray, J. A. Koch, C. Lee, D. Kern, R. A. London, B. J. MacGowan, D. L. Matthews, and G. Stone, Science 258, 269 (1992).

22. L. B. Da Silva, B. J. MacGowan, S. Mrowka, J. A. Koch, R. A. London, D. L. Matthews, and J. H. Underwood, Opt. Lett. 18, 1174 (1993). 


\section{Section IX}

\section{Radiative Properties}

The study of radiative properties using highenergy lasers has a long history. The irradiation of a surface with a high-energy laser provides copious $x$-ray emission that is easily accessible and inexpensive.

Over the years the development of $x$-ray spectroscopy has led to further inquiries into the origin of the radiation, kinetics processes of importance, and radiative transfer in these plasmas. These are all grouped here for discussion. Some of the topics summarized below are quite complex, while others are dealt with in a more schematic manner. This difference in treatment is due to the varying levels of sophistication we now have in the various areas.

There are various constraints on experimental aspects of the problem of radiative properties. In order to cover these constraints in a coherent way, the background material on radiative properties has been grouped so that each topic builds on the previous discussions. Thus, the topics are grouped into five subsections:

A. Atomic Physics and Isolated-Emitter Spectroscopy

B. Plasma-Emitter Radiative Properties

C. Dynamics Properties

D. Plasma Spectroscopy

E. Radiative Transfer

The various ideas for the study of radiative properties in hot, dense matter or high-energydensity conditions fall naturally into these five areas. The areas can be traced to the various degrees of complexity that the surrounding matter imposes on the radiator. For example, at the first level (Subsection A, Atomic Physics and
Isolated-Emitter Spectroscopy) we are only interested in the isolated ionic emitter, and the fact that there are interactions is significant only in that the interactions serve to create the highly ionized or specially populated ionic radiator. The spectroscopy of highly stripped ions falls into this category.

Subsection B, Radiative Properties of Plasma Emitters, goes into the effect of the plasma . environment on the emitter.

Subsection C, Dynamics Properties, covers the dynamics, or evolution, of the radiator in the plasma.

Subsection D, Plasma Spectroscopy, then discusses measuring quantities relevant to these $x$-ray- or laser-produced plasmas.

And finally, Subsection E, Radiative Transfer, covers the transport of radiation, first for conditions of local thermodynamic equilibrium (LTE), and then for plasmas that do not satisfy the conditions for LTE, which is a more complex situation.

\section{A. Atomic Physics and Isolated-Emitter Spectroscopy}

The production of highly stripped ions is not a straightforward extrapolation of laser energy. For example, in small 100-joule 1-ns-type lasers, it is possible to reach ion stages of $\mathrm{K}$-shell titanium. Further, the 24-beam Omega laser, which delivered 50 joules per beam, was used to probe transitions between the $4 n$-type ground configuration and any excited configuration over the entire periodic system. 
The limits for some other transitions are:

- $2 \mathrm{~s}^{2} \mathrm{~S}_{1 / 2}-2 \mathrm{p}^{2} \mathrm{P}_{1 / 2,3 / 2}$ and $2 \mathrm{p}^{2} \mathrm{P}_{1 / 2,3 / 2-}$

$3 \mathrm{~d}^{2} \mathrm{D}_{3 / 2,5 / 2}$ transitions in lithium-like

systems.

Current knowledge: $\mathrm{Ge}^{29+}(Z=32)$

Source for highest degree of ionization:

Omega

Degree of ionization reached with the

Omega laser: $\mathrm{Ge}^{29+}$

- $2 s^{2} 2 p^{5} 2 P_{3 / 2,1 / 2}-2 s p^{6}{ }^{2} S_{1 / 2}$ transitions in

fluorine-like systems.

Current knowledge: $\mathrm{Sn}^{41+}(Z=50)$

Source for highest degree of ionization:

Nova 2-beam

Degree of ionization reached with the

Omega laser: $\mathrm{Sn}^{41+}$

- $\quad 3 s^{2} \mathrm{~S}_{1 / 2}-3 \mathrm{p}^{2} \mathrm{P}_{1 / 2,3 / 2}$ and $3 \mathrm{p}^{2} \mathrm{P}_{1 / 2,3 / 2}-3 \mathrm{~d}$

${ }^{2} \mathrm{D}_{3 / 2,5 / 2}$ transitions in sodium-like

systems.

Current knowledge: $\mathrm{Gd}^{53+}(Z=64)$

Source for highest degree of ionization:

Nova 2-beam

Degree of ionization reached with the

Omega laser: $\mathrm{Sn}^{39+}$

However, it is clear that using ever-higher laser energies in a straightforward application of direct target irradiation will not necessarily permit access to yet higher ionization stages. The difficulty is that much of the additional energy will go into kinetic directed energy and the coupling efficiency will be relatively small. One solution for this is the use of the rather newly perfected "gas-bag" targets. These are simple targets made of a thin plastic bag, currently containing large molecular structures of low- $Z$ atoms. This allows the ambient pressure of the bag to be low ( $\geq 1 \mathrm{~atm}$ ), while the fully ionized targets can have electron densities on the order of $10^{21} \mathrm{~cm}^{-3}$. These are described in more detail in Section VII, Plasma Physics.

The study of the atomic physics of highly ionized atoms came into the modern era with the availability in the late 1960s of reasonably high-energy lasers. The production mechanism for obtaining ionized material on a high-energy laser is straightforward: irradiate a solid surface of the element of interest with a laser at intensities greater than the surface melting point. This technique has produced spectra and line identifications of numerous highly ionized species.

The method has been improved with the use of a localized dot of the material of interest in a matrix of a lower- $Z$ material, which will not interfere spectroscopically. This helps isolate the element of interest to a finite column that will move outward along the laser axis from the solid surface. The dot spectroscopy method is schematically illustrated in Fig. IX-1. ${ }^{1}$

The dot spectroscopy technique has been widely used to improve spectral resolution (which can be compromised by broadening due to the finite source size), and to permit spectral information from spatially resolving instruments to isolate regions of the plasma in which there are small temperature and density variations.

Along this line we mention two results. First, we have the work of Seely and others performing classical line identification on higher- $Z$ elements. In this work a survey soft $x$-ray spectrometer with wide spectral coverage is used to look in the XUV region. Results of this technique have been used to obtain, for example, the configuration interaction and level crossing data relevant to the $3 \mathrm{~d}^{10} 4141^{\prime}$ levels in highly ionized zinc-like atoms with atomic numbers from 40 to $80 .^{2}$ These experiments are used to provide difficult-toobtain data.

Second, the identification of highly stripped ions in laser-produced plasma can lead to interesting diagnostic possibilities. For example, the density-sensitive emission line ratio from holmium XXXIX has been inferred from an experiment where the XUV spectrum can be both spatially and spectrally resolved. ${ }^{3}$ Fig. IX-2 shows an intensity-vs-wavelength plot of the lines of holmium XXXIX at one spatial position. When a temperature of $1780 \mathrm{eV}$ and an optically thin plasma are assumed, various ratios of these emission lines form a reasonable diagnostic. This is illustrated in Fig. IX-3, which shows the ratios of various line emission intensities vs electron density.

The final comment on spectroscopy of laserproduced plasma is to point out the interest in 
Front view

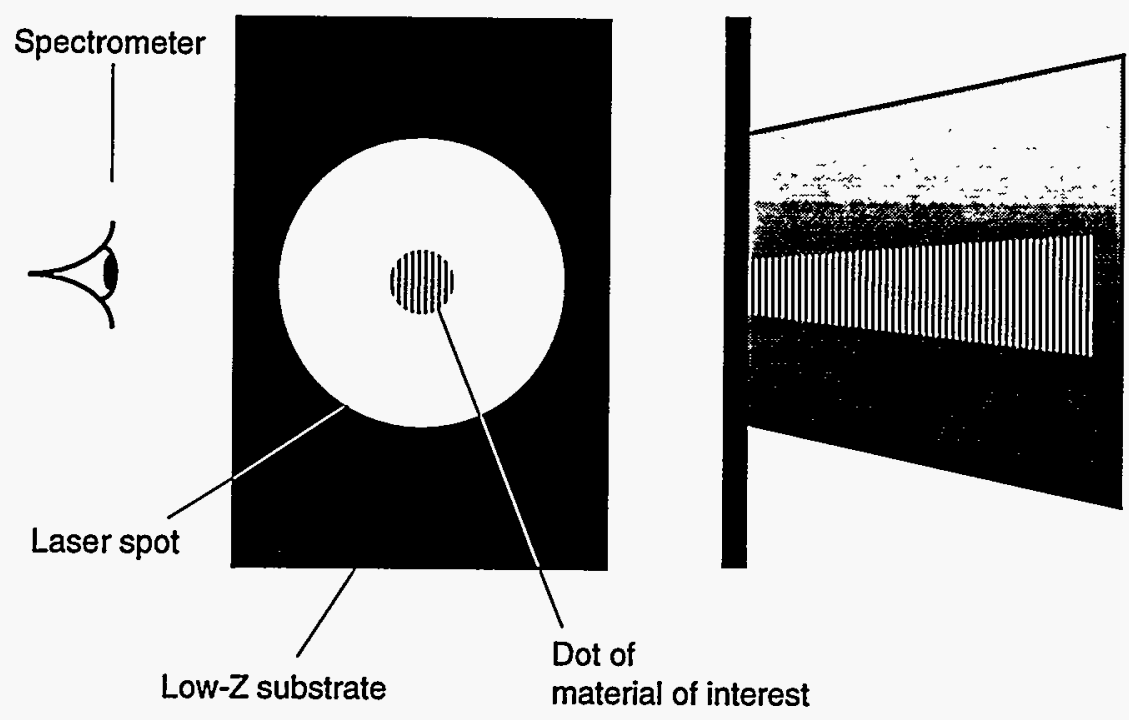

Figure IX-1. Schematic of dot spectroscopy technique. The dot of the material of interest is placed on, or slightly beneath, a target that will not interfere spectroscopically. When the surface is irradiated, the dot is confined, reducing the spatial variations possible.

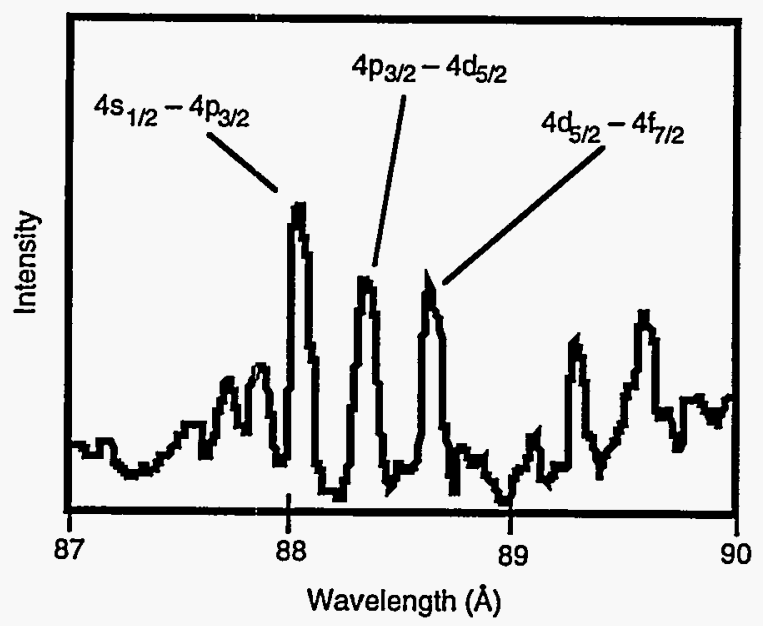

Figure IX-2. Example of the spectrum of lines of holmium XXXIX $n=4-4$ lines at one spatial position. The lines used in Fig. IX-3 are indicated here.

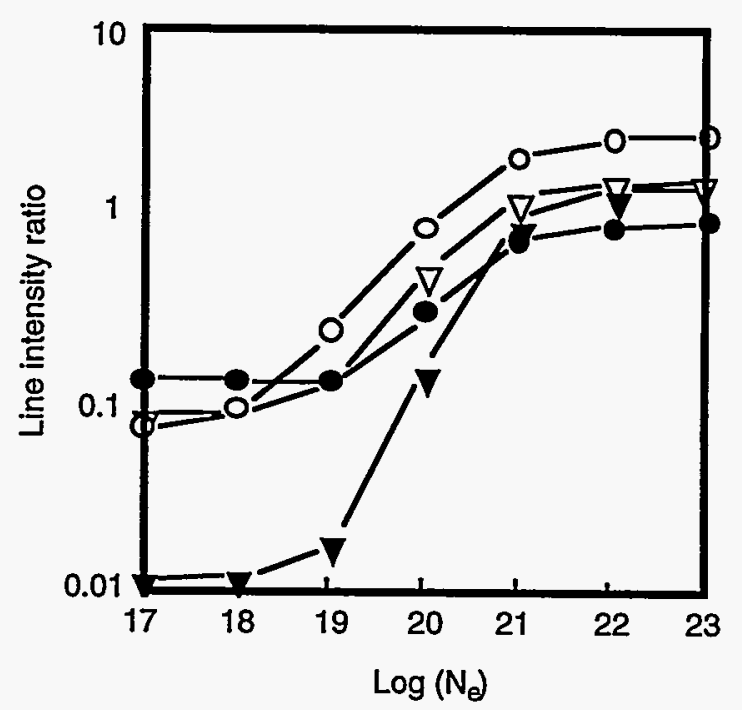

Figure DX-3. Ratios of various line emission intensities:

$$
\begin{aligned}
& u={ }^{2} \mathrm{P}_{3 / 2}-{ }^{2} \mathrm{D}_{5 / 2} \text { to }{ }^{2} \mathrm{~S}_{1 / 2}-{ }^{2} \mathrm{P}_{3 / 2} \\
& t={ }^{2} \mathrm{D}_{5 / 2}-{ }^{2} \mathrm{~F}_{7 / 2} \text { to }{ }^{2} \mathrm{~S}_{1 / 2}-\mathrm{P}_{3 / 2} \\
& o={ }^{2} \mathrm{P}_{1 / 2}-\mathrm{D}_{3 / 2} \text { to }{ }^{2} \mathrm{~S}_{1 / 2}-{ }^{2} \mathrm{P}_{3 / 2} \\
& \cdot={ }^{2} \mathrm{D}_{5 / 2}-{ }^{2} \mathrm{~F}_{7 / 2} \text { to }{ }^{2} \mathrm{P}_{3 / 2}-\mathrm{D}_{5 / 2}
\end{aligned}
$$


the structures called unresolved transition arrays (UTAs), which are spectral features composed of myriad line transitions from complex ions. These structures provide a wealth of information on the plasma formation and atomic physics of complex atoms. ${ }^{4}$

\section{B. Plasma-Emitter Radiative Properties}

To study the effect of the plasma environment on the emitter, you must create a plasma and the ionic emitter in a condition that is wellcharacterized. A special plasma-production mechanism is required to study the intrinsic profile of a line transition, the shape of the resonances in a line series leading up to the ionization potential, the interaction of the discrete resonances with the underlying continua that occur above the first ionization potential of an ion, or the absolute shape and intensity of the continua.

In the past, the production of the ionic emitter in a hot and dense plasma and verifying its existence was of sufficient scientific interest to permit the use of relatively simpler plasmacreation strategies; for example, the irradiation of a solid target. However, when the goal is to disentangle the temporal and spatial variations of the plasma in order to determine the fundamental radiative properties of an ion immersed in a plasma, a method must be found to determine the plasma conditionstemperatures, densities, etc.

Determining the conditions of a plasma requires systems that are sufficiently steady-state and, ideally, have no gradients. The steady state is required to ensure that the radiative property is not a function of the time history of the system, but of the instantaneous conditions. The spatial constraint (i.e., no gradients) comes from the fact that critical determination of the effects of interest requires that the contribution from widely varying plasma conditions be minimized. As a simple example, in order to determine the existence (or non-existence) of a transparency window between the last existing line in a series and the associated bound-free continuum, it must be possible to ensure that there is no filling of the proposed window due to the existence of plasma at slightly different conditions.

Generating this type of single-density, singletemperature plasma is highly energy intensive. First, direct irradiation of a sample will produce large gradients. Second, the problem with gradients has not to date been remedied by the use of microdots of the element of interest embedded in a solid material. Direct irradiation would therefore seem to be a poor method for obtaining the test-bed plasma for studying plasma-emitter radiative properties.

However, there are experiments that may indicate a way to create the ideal system. A great deal of work has been performed in the characterization of the opacity of elements with low- and middle-range Zs. This work indicates that the entire capability of Nova is required to achieve tens of electron volts at 0.001 of solid density.

The reasons why the generation of singledensity, single-temperature plasmas requires the entire capability of Nova are clear. First, the material from which the plasma is created must be volumietrically heated. Second, this volumetric heating is most easily achieved by creating an $x$-ray flux. And third, the sample must be hydrodynamically isolated. (Hydrodynamic isolation keeps the sample from suffering shocks, etc., from laser deposition or indirectly from collision with laser and $x$-ray ablated material.)

The two processes of up-conversion of laser light to $x$-rays and the volumetric deposition of the $x$-rays reduce the amount of energy reaching the sample, resulting in less than $1 \%$ of the initial laser energy being coupled into the sample. The need for hydrodynamic isolation, implying separation of the sample and $x$-ray source, causes the coupling to drop further by an additional multiplier that will be less than 0.1 .

These factors together result in less than $10^{-3}$ coupling of the initial laser energy into the material of interest. Experience in the use of hohlraums and x-ray-irradiated samples, therefore, leads to a simple scaling for the production of characterized samples-that we are limited to temperatures well below $100 \mathrm{eV}$, and probably $50 \mathrm{eV}$ is the maximum currently. It is 
therefore implicit in all the discussions of the effects that we wish to study that the greatly increased energy of the NIF will allow us to extend our range of temperatures and densities toward the higher- $Z$ materials and regimes of importance.

\section{Dynamics Properties}

The next level of detail is the study of the dynamics, or evolution, of the radiator in the plasma. At one level, the effects discussed above of the plasma on the imbedded emitter are a form of kinetic process. By dynamics we mean here the actual time dependence of the detailed ionic state populations (i.e., the result of the rate equation solution for the populations). The plasmaaffected radiative properties (e.g., line shapes) discussed above require a plasma averaging that can, by the ergodic hypothesis, be replaced by a time average. The time average then indicates a dynamic process; however, it is one where the averaging process replaces the actual temporal history. In looking at the dynamics of the radiator, we are interested in the details of the time histories, population densities, and population mechanisms.

In studies attempting to isolate the dynamical properties of a hot, dense plasma, numerous processes play a role. The formation of atomic models can require exhaustive detail, and this in itself has become a topic of research over the past decade. That is, some states of the atom may have relatively little observable contribution to the spectrum, but play an important part in the flow of population. This makes the task of kinetics modeling difficult, insofar as the observables are dark shadows providing only a vague sense of the processes required to create the populations.

In studies of dynamical properties the open questions include difficulties with the truncation of high-principal-quantum-number states, the inclusion of states that have auto-ionization channels, and the level of detail for the states. The most obvious example where these considerations are critical is for the prediction of inversion and laser gain in rapidly varying systems. However, the evolution of the radiators in hot, dense matter in general is of great importance.

The additional constraint on the experimental setup, beyond those discussed above in reference to radiative properties, comes from the ability to probe, in time, the level populations that may take part in the formation of the populations. This potential creates an interest in developing the ability to probe states that may not be seen in emission. The development that will be required is the ability to probe states and transitions by methods that are similar to those that we require for radiative properties (discussed above) and those required for radiation transfer (as discussed below), but with the additional fact that the plasma characterization must be performed in a time-dependent manner. Determination of radiative properties from a single-temperature and -density plasma may provide definitive information; however, this is only a part of the puzzle when it concerns the flow of population density through the various ion species.

A critically important advance would be the addition of a probe that could be used to generate fluorescence from excited states. Developing such a probe is difficult. With low-energy-density plasma, the equivalent to this experiment requires lasers as the pumping source. In the high-energy-density regime, the production of appropriate short-wavelength lasers is not an easy task, and to date no line coincidences have been located for use of the existing soft $x$-ray lasers as pump sources.

Thus, we await, in the current generation, an appropriate source. With the advent of the NIF, photon densities from quasi-continuous pseudoPlanckian sources could be spectrally filtered to provide the necessary pumping for fluorescence and pump-probe experiments. Until such time as a source of the magnitude of the NIF becomes available, we will have to rely on the search for coincidences with existing bright laser-plasma sources, or the use of high-emissivity line radiation from spatially separate plasmas, to provide a photon pump of the same transitions in another plasma. 


\section{Plasma Spectroscopy}

The plasma produced by directly irradiating targets gives rise to states that are well outside of the defined limits of the condition of LTE in the plasma. The time dependence of the heating source, together with the fact that the plasma expands rapidly during heating by the laser pulse, indicates that these plasmas must be studied using non-LTE (NLTE) techniques. This, in turn, means that atomic models coupled to NLTE rate equations must be employed to predict the populations in the plasma, and thus these models are necessary for interpretation of the spectral character of the plasma emission in general. The further complication that the plasmas are spatially varying indicates that the hydrodynamics of the plasma must also be modeled for a fuller understanding of the plasma.

To understand laser-produced plasmas, much effort has been expended to develop a diagnostic complement that will provide data independent of plasma simulations. These diagnostic methods include development of the instruments (which is discussed in Section III, Experimental Capabilities), as well as of plasmaspectroscopic diagnostics. Many of these diagnostics are complementary to those developed for astrophysical, atmospheric, or other laboratory plasmas.

One of the methods that has found great success is the previously mentioned dotspectroscopy technique. This technique has been perfected so that the temporal, spatial, and spectral information is obtained on a single shot, and it can also provide independent measures of, for example, electron density. A schematic of such a setup is shown in Fig. IX-4..$^{5}$ Figure DX-5 shows the results for the electron temperature measurement that uses the slope of the boundfree emission continuum of the hydrogenic species. This experiment represents the type of detailed spectroscopy that can be performed on these plasmas even though they are rapidly evolving during the experiment.

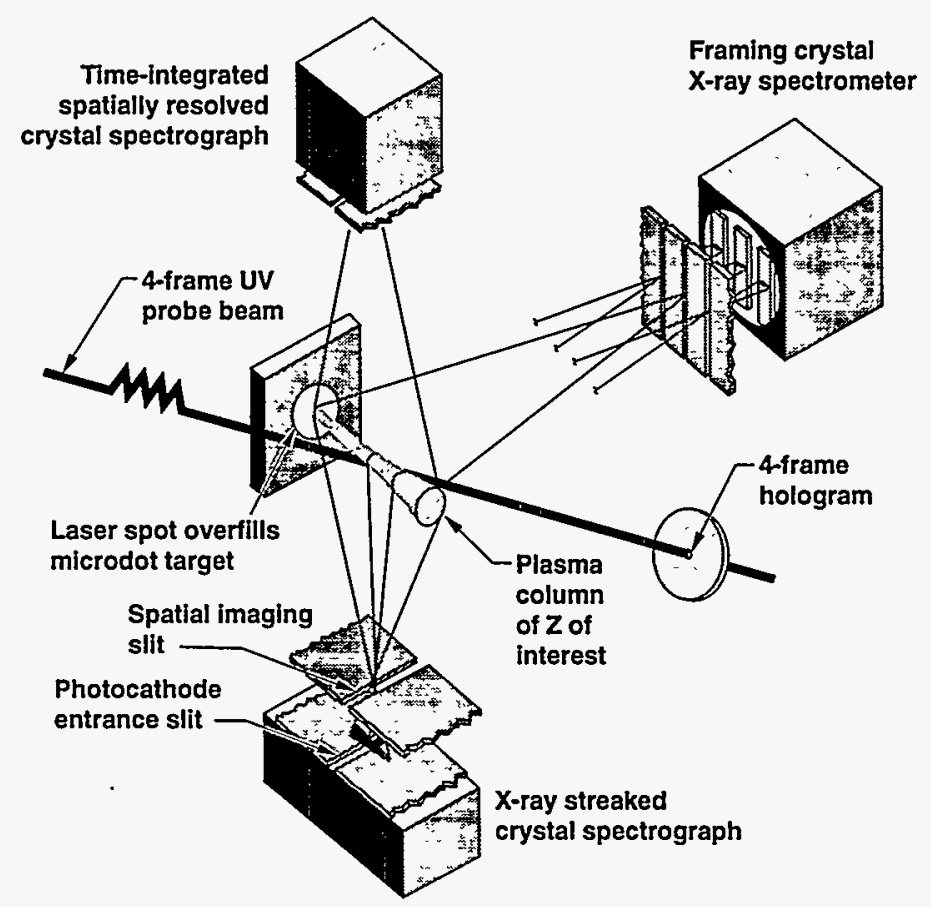

Figure IX-4. Schematic of a spectroscopy experiment that provides time, space, and spectral information. In this experiment a holographic interferometer is used to also provide an independent measure of the plasma electron density. 


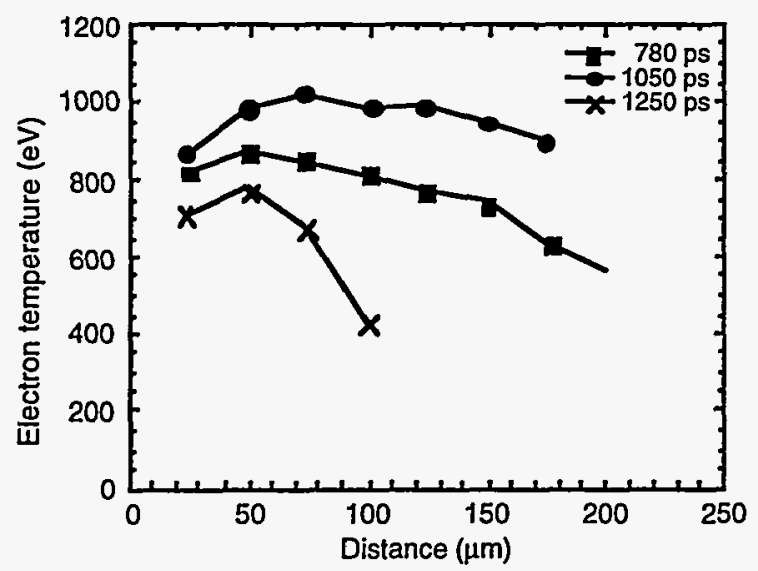

Figure IX-5. Temperature measurements from a spectroscopy experiment of the type shown schematically in Fig. IX-1. The temperature from the hydrogenic bound-free recombination continuum is measured for several frames on a spatially resolving spectrometer.

This brings us to an important point concerning the laser irradiation of a surface. The plasma that is formed during the laser-matter interaction will be dense and warm at the surface, becoming tenuous and hot as the matter moves away from the target. Therefore, defining the temporal and spatial history of the material is a challenging feature of these experiments. This, as one might expect, gives rise to a field of study of its own.

One rather elegant advance was to use a series of concentric dots of differing elements, bull's-eye fashion (to isolate the spatial information in the direction perpendicular to the laser axis), and then to use a gated spectrometer that provided spatial resolution along the laser axis. In this form of the experiment the NLTE character of the plasma can be both analyzed and at the same time used for simple line identification.

The methods developed for use on laserproduced plasmas include the standard line intensity ratio techniques, the use of spectral line broadening, and most recently, a novel but simple extension of the line ratios technique to the use of two different elements. ${ }^{6}$

This new idea is illustrated in Fig. IX-6, which shows the ratios of the helium-like $1 s^{2}$ $1 s 3 p$ transitions from titanium to chromium in the same plasma conditions. Figure IX-7 shows the results of an experiment on a long-scalelength plasma created by irradiating a bag of gas that is underdense. That is, when fully ionized, the electron density $\left(\mathrm{N}_{\mathrm{e}}\right)$ of the gas is less than the critical density $\left(\mathrm{N}_{\mathrm{C}}\right)$ for the laser frequency used. The laser heats the gas to a few kilovolts, and the chromium/titanium line ratios are a good diagnostic of this temperature. The line emissions are obtained from chromium- and titanium- coated fibers introduced into a bag of $\mathrm{n}$-pentane having an $\mathrm{Ne}_{\mathrm{e}}$ of $10^{21} \mathrm{~cm}^{-3}$. The chromium and titanium line ratios in Fig. IX-7 indicate that the $\mathrm{T}_{\mathrm{e}}$ is $\sim 3 \mathrm{keV} .^{7}$

In terms of novel diagnostics, measurement techniques have been developed that employ line shapes, ${ }^{8}$ inner-shell absorption techniques, ${ }^{9}$ and quasi-steady-state models, ${ }^{10}$ as well as $\mathrm{x}$-ray laser probes. ${ }^{11}$ Some these will be discussed below in Subsection F, Future NIF Experiments, under "Plasma Spectroscopic Topics."

\section{E. Radiative Transfer}

One of the unique capabilities of high-energy lasers is that they can reach sufficient energy density, albeit for short times, to permit the study of radiation transfer as a topic in its own right. Since the transport of radiation plays an integral part in stellar interiors, as well as in inertial confinement fusion (ICF) implosions, this has become an area of some interest.

There are clearly two general regimes. In the first regime, when the transfer of radiation occurs in material that satisfies the conditions for local thermodynamic equilibrium, the transfer is studied as a flow of radiation through the material. In these experiments we are interested in measuring flow characteristics as these are represented by quantities such as the Rosseland opacities and described by the radiation diffusion approximation.

The second regime deals with plasmas that do not satisfy the conditions for LTE, and the demands become somewhat more complex. Whereas in LTE we can use the Saha-Boltzmann equation or some related statistical method to obtain populations, in NLTE cases we require atomic models of some sophistication that 


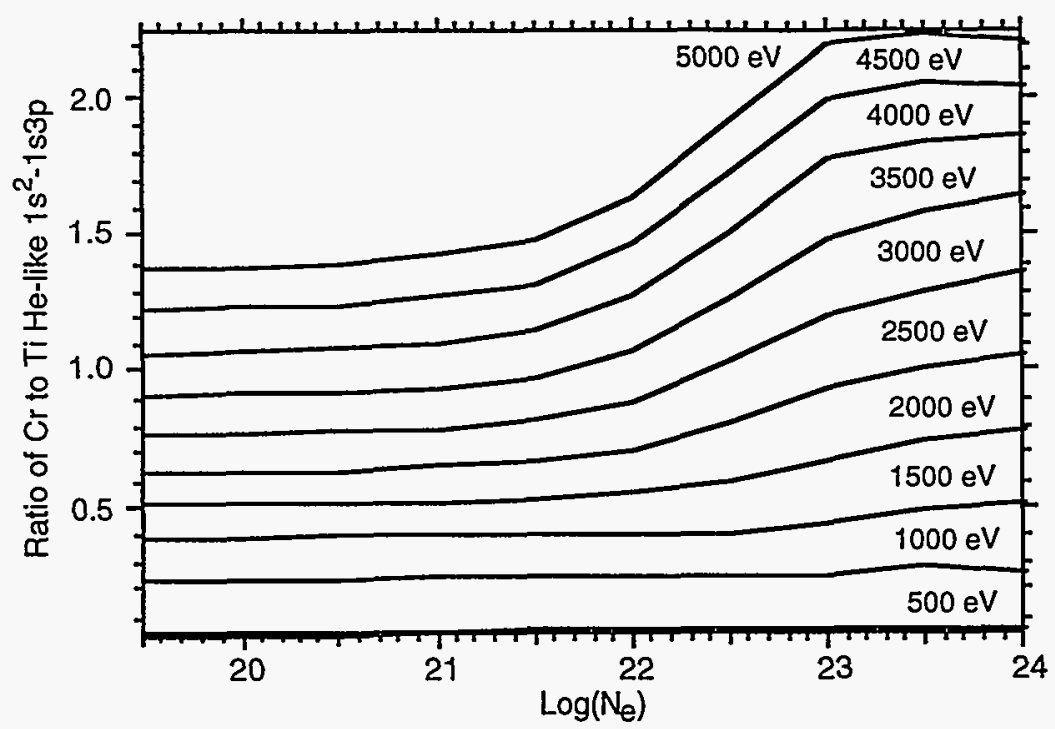

Figure IX-6. Ratio of the chromium to titanium helium-like $1 s^{2}-1 s 3 p$ intensities for a range of temperatures and densities. Note that each curve represents a single temperature.

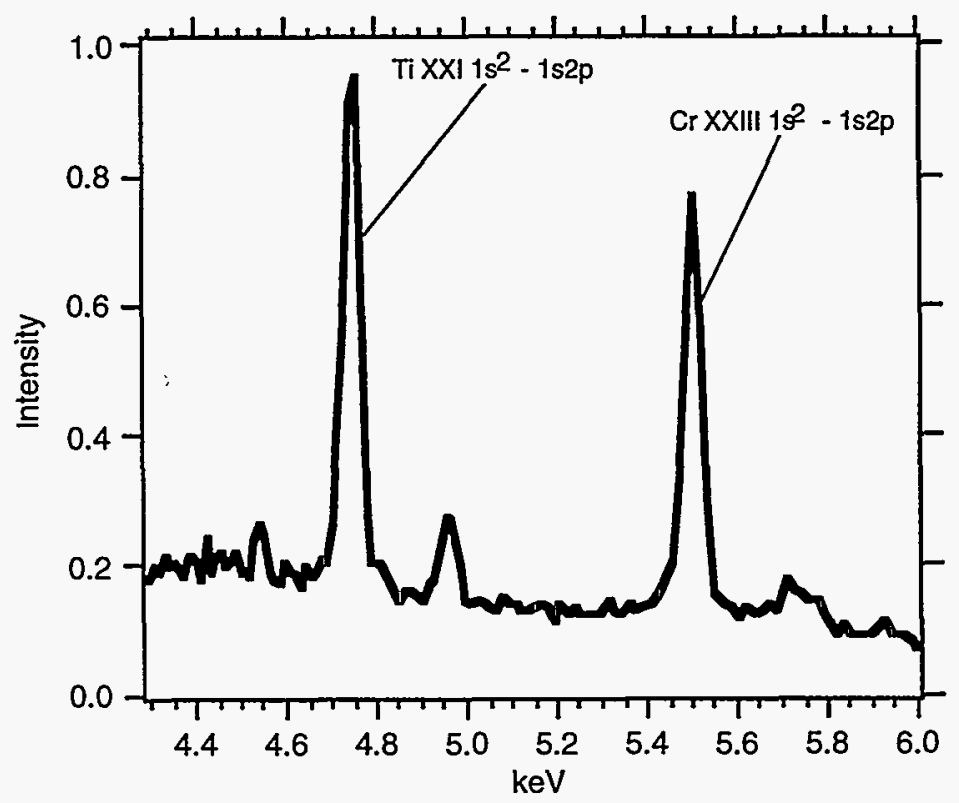

Figure IX-7. A spectrum of the chromium and titanium produced by irradiating a large bag of gas with a single 3-ns long pulse of 3600-J energy. The chromium and titanium are on fibers introduced into a bag of $n$-pentane, yielding an $\mathrm{Ne}_{e}$ of $10^{21} \mathrm{~cm}^{-3}$. The line ratios indicate the $T_{e}$ is $\sim 3 \mathrm{keV}$. Because the electron density of the gas is relatively undisturbed, due to the small amount of hydrodynamic motion, temperature can be determined. 
demand rates for all the processes of importance. In addition, the radiation diffusion equation is no longer useful, and we must solve the detailed radiative transfer equation that, in general, must be solved self-consistently with the rate equations.

\section{LTE Radiation Flow}

Currently there is a series of experiments to study LTE radiation flow-the transfer of heat by $x$-rays through targets of varying types. The basic experimental geometry is shown in Fig. IX-8. The $x$-ray drive for these experiments is created by focusing eight of the ten laser beams at Nova into a gold hohlraum. The $x$-rays from the hohlraum are incident on one end of a millimeter-scale tube containing one of several possible experimental packages (see Fig. IX-9). From the opposite end of the tube the backlight passing through the package is observed with an $x$-ray crystal coupled to an $x$-ray streak camera.

Backlighting illumination is created using the other two Nova beams. Behind the radiation flow target is a tiny fiber of samarium that is heated by laser light. This produces a broad-band $x$-ray backlighting spectrum at photon energies from around 1 to $2 \mathrm{keV}$.

Figure IX-9 shows the various possible targets used in the radiation flow experiments. (Hohlraums represent a feature of these, and many other, experiments. For more information about the hohlraum, see Section III, Fig. III-1 and Fig. III-2.)

The tube contains a series of thin fiducial foils that register the transfer of heat by allowing us to observe the absorption spectra of $\mathrm{K} \alpha$ transitions in the foils. As the fiducial foils heat, they go through several different ionization stages. Each

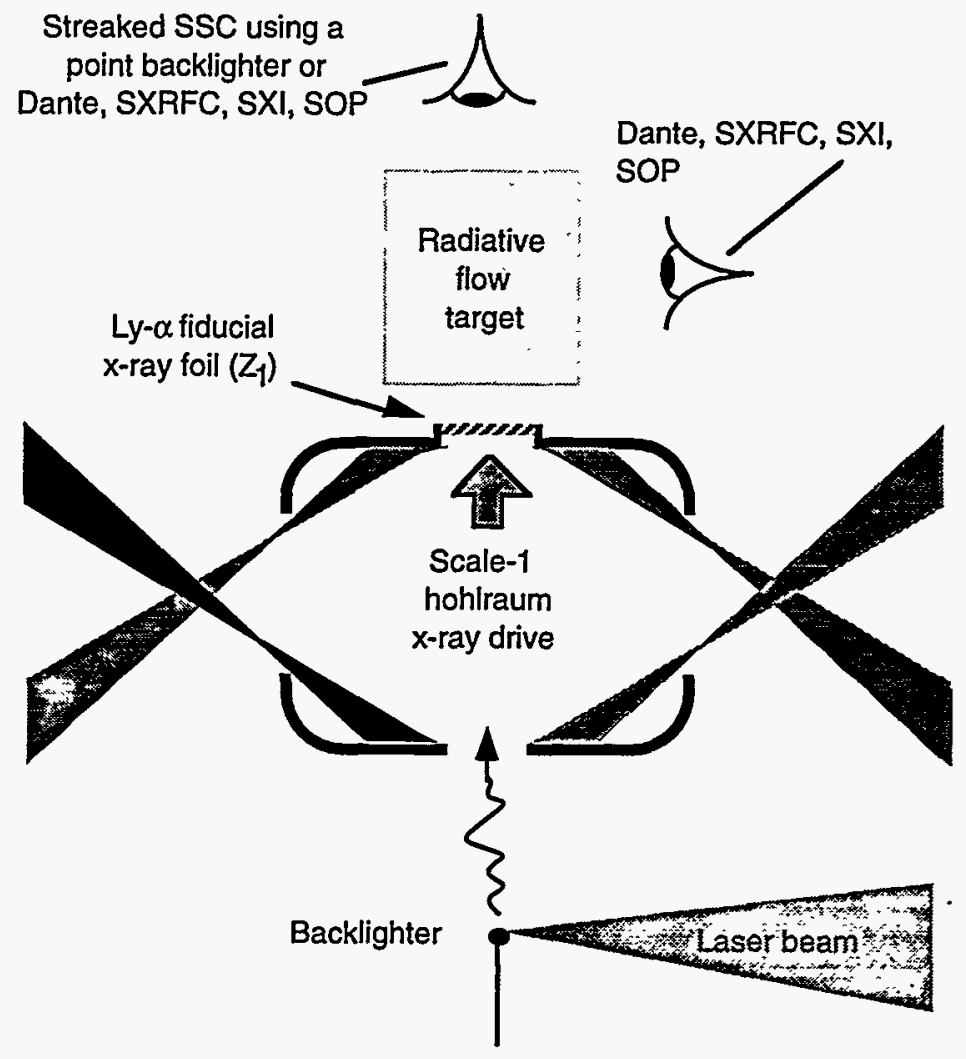

Figure IX-8. Schematic of radiative flow experiment. $X$-rays from a Nova hohlraum are incident on one end of a millimeter-scale tube containing an experimental package. Eight of Nova's ten beams are focused on the hohlraum; the remaining two are used for the backlighter. (See Section III for instrument names.) 

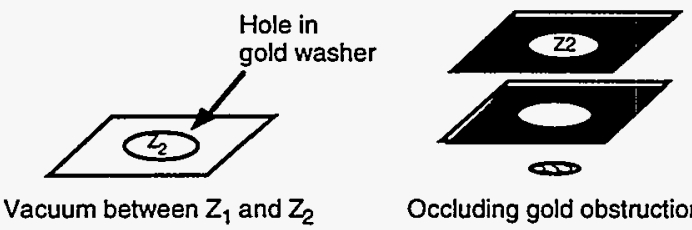

Occluding gold obstructions

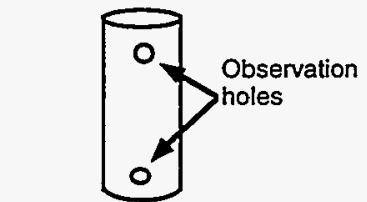

Empty or foam-filled tube

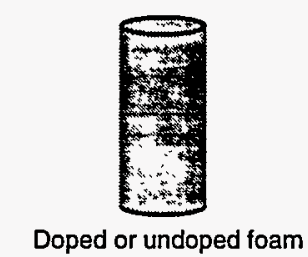

Figure IX-9. Possible experimental packages for radiation flow experiment shown schematically in Fig. IX-8.

ion stage absorbs different frequencies from the $x$-ray backlighting spectrum. The streak camera records the ionization history of each of the fiducial foils in the tube. From this, the time history of the temperature of each foil can be inferred. ${ }^{12}$ Fig. IX-10 presents an example of a streak-camera data record for radiation flow through a tube.

Both one-dimensional burn-through foils and more complicated two-dimensional geometries are being studied. The initial $x$-ray driving flux has been well characterized in terms of its time-, angle-, and frequency-dependence. Heat-transfer experiments are also being performed using a two-dimensional $x$-ray framing camera to study the longitudinal distribution of heat in both unobstructed and baffled straight cylindrical geometries.

These initial experiments have also been extended using smaller hohlraums that create hotter radiation sources capable of driving longer and more complex two-dimensional geometries. The radiative drives from these hotter sources have also been well-characterized.

\section{Non-LTE Radiative Transfer}

Interest in the generation of $x$-ray lasers led to a series of relatively recent advances in theory and experiment. These advances have brought us to a position where detailed radiation hydrodynamics simulations are available and

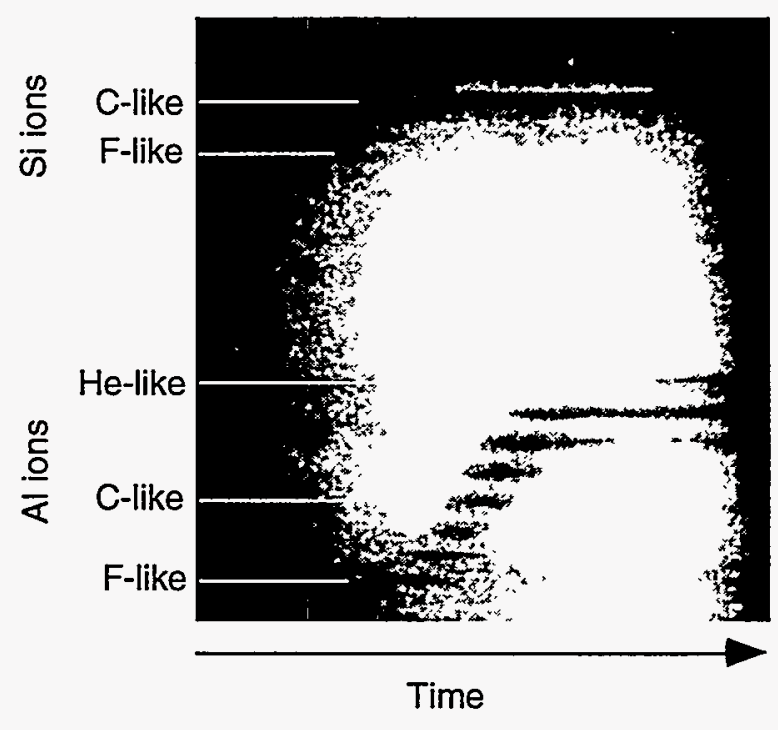

Figure IX-10. Streak-camera record of backlight passing through a radiation-flow package in the experimental setup shown in Fig. IX-8. Time runs horizontally and the spectrum is vertical. At early time the silicon $K \alpha$ lines are seen. As the radiation flows up the tube, at late time, the aluminum $K \alpha$ lines rise up.

detailed kinetics models can be produced, with some effort. (It is important to note the central role of the astrophysicist in the development of these simulation capabilities. Although the largest part of the motivation for the experiments shown here was derived from interest in laboratory plasma sources, astrophysicists were producing the methodology for doing NLTE radiation transfer "correctly.")

Thus, there are simulation codes that now provide a radiative-hydrodynamics capability for the analysis of NLTE experiments. A description of the basics of these codes is not necessary here, but the fact remains that these codes exist and that they have been developed at great expense. Rigorous testing of the codes has essentially been confined to benchmarking numerical problems where analytic solutions exist, and no rigorous tests have been performed against controlled high-energy-density plasma experiments.

The first goal of the effort on NLTE radiative transfer will be to develop a method to test the simulation codes by making it possible to compare the results of radiative hydrodynamics 
simulations with experimental results. This method must go as far as possible towards allowing detailed comparisons to be performed. We must be able to provide the initial conditions to the code more accurately than has been previously attempted, and then provide the results of the experiment. The hope is to provide experimental results in the form of absolute intensities so that the details of simulations can be tested for the first time.

More specifically, there are three areas in which it is felt simulation capabilities should be tested. First, the accuracy of the atomic kinetics models will be measured insofar as we can provide spectral information of high enough quality to check model details.

Second, the suitability of the kinetics models in simulating plasma dynamics can be tested. This testing will be made possible by the ability to obtain experimental information on the evolution of the radiatively driven system.

Third, and most difficult, the level of information that will be available on a NIFscale facility may be sufficient to constrain the radiation transfer algorithms in these timedependent plasmas with multilevel atomic systems.

Note that the testing of radiative transfer would require the generation of spectra that contain line transitions formed in a radiatively dominated regime. This is a very difficult regime to attain in the laboratory, as can be seen from the simple rule of thumb that for radiation to dominate the collisions in the line formation process, the following must be true:

- The $\varepsilon$ parameter (defined as the ratio of the collision decay to the radiative decay of a transition) must be small compared to unity

- The optical depth $\tau$ of the transition must be large

- The source function $S$ should reach the blackbody limit, $B$.

The $\varepsilon$ parameter can be evaluated as $\varepsilon \approx 10^{-4} N_{\mathrm{e}} \lambda^{3}$

where $\lambda$ is the transition wavelength in $\mathrm{cm}$ and $N_{\mathrm{e}}$ is the electron density in $\mathrm{cm}^{-3}$. (Here we assume that the Gaunt factor is 0.2 and the plasma temperature is $100 \mathrm{eV}$.)
Next, the optical depth, $\tau$, of a Doppler transition can be approximated by assuming that the oscillator strength is $\approx 0.5$, the temperature is $\approx 100 \mathrm{eV}$ and the atomic number is $\approx 20$. Then

$$
\tau \approx 6.3 \times 10^{-10} \mathrm{~N}_{\mathrm{g}} \lambda \iota
$$

Here lis the plasma column length in $\mathrm{cm}$ and $N_{\mathrm{g}}$ is the ground-state number density.

If we want the line formation to work in a regime where the source function reaches the blackbody limit (so that the medium is effectively thick), we can relate $\varepsilon$ to $\tau$ by the equation

$$
S_{\max } / B \approx \varepsilon \tau \sqrt{\ln (\tau)}
$$

for Doppler line profiles. Analysis indicates that, for example, a column length of $10 \mu \mathrm{m}$ of plasma with an electron density of $10^{20} \mathrm{~cm}^{-3}$ and $10^{19}$ $\mathrm{cm}^{-3}$ ground-state ions will yield (for 100- $\AA$ radiation) an $\varepsilon \approx 0.01$, a $\tau \approx 6.40$, and a maximum source function near the blackbody limit.

These conditions are difficult to attain, but are possible in the type of experiments that are currently being performed. The main object of this work to obtain a test bed for radiative transfer experiments, therefore, would be to develop the techniques to perform measurements in such a plasma. Detailed studies of parameter regimes where $\varepsilon$ is much smaller and/or $\tau$ is much larger are important to the verification of the radiation transfer schemes, but the goal of the current experiments is to create an experimental environment in which radiation dominates the collisions in the line formation process so that detailed studies can eventually be performed. But, first things first.

The progress so far on the current experiments is as follows. First, based on several considerations, an experimental configuration is now being tested. The considerations are:

- The use of radiation enclosures (i.e., hohlraums) is not necessary for the production of NLTE experiments. The enclosure provides nothing of interest to the experimental design, and in fact complicates the measurement process to the point of rendering the experimental plan all but unattainable.

- The use of direct-drive experiments, although of great interest to the community of experimentalists as a fertile source of ideas and developments, 
produces a plasma that is heated by mechanisms not relevant to the testing of the NLTE radiative hydrodynamics simulations.

- The dimensionality of the experimental system must be reduced to the minimum so that we can provide, as nearly as possible, an ideal NLTE system. This is achieved by using a low- $Z$ tamping layer to contain the material of interest.

- Because the temperatures that can be reached with current facilities are well below the kilovolt range, spectral measurements should be made in the sub-kilovolt range. This will require an effort to develop XUV instruments.

Given these considerations, the experiment can be defined. A schematic of the experiment to study NLTE phenomena as it is being fielded is shown in Fig. IX-11.

The experimental plan is now going forward on three levels. First, we have developed a full understanding of the radiation heating source used in these experiments. The source, which arises from a gold burn-through foil, has been measured in time, spectrum, and angle (for more information see Section VIII, Radiation Sources). ${ }^{13}$

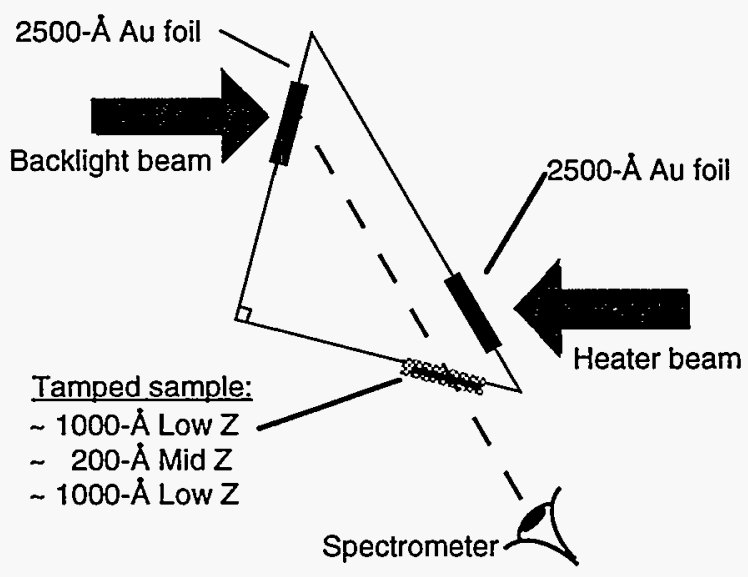

Figure IX-11. Schematic of setup to study NLTE phenomena. Heating is achieved by using the flux from the rear side of a gold burn-through foil. The sample in this case is tamped, and the backlight can be delayed in time for the heating.
Second, we have developed methods to perform the absorption spectroscopy of the heated samples. Figure IX-12 shows an example of the spectrum from an untamped boron nitride target. The figure shows a comparison of the theoretical absorption spectrum with the experimental results for this case. The boron nitride sample is untamped, so effects due to the vacuum/matter interface will be present. Note that this sample should not come too far out of LTE, and comparisons have been performed that indicate that this is true.

The third step is spectroscopy of the tamped sample and the relevant characterization of the material with small gradients. In Fig. IX-13 we see the experimental results from first attempts at measuring a Stark-broadened absorption line profile to ascertain $N_{\mathrm{e}}$ as a function of time. The results are for absorption of a gold backlight through a plastic-tamped Teflon sample. The figure shows both the measured lithium-like fluorine and the line shape predicted by theory, and includes the densities inferred from the measured results. Density can be determined as a function of time by using a long-duration backlight (i.e., absorption source) with an XUV streak camera. Then the time-dependent width of the absorption line yields a time-dependent density. The development of in-situ temperature diagnostics and further density diagnostics is being evaluated.

\section{F. Future NIF Experiments}

\section{Spectroscopy of High-Z Elements}

To provide a background to assist in understanding the importance of experiments using high-energy lasers, we will describe the current state of knowledge of highly ionized atoms as it relates to some key isoelectronic sequences. In particular, this description will help in extrapolating those experiments to the NIF.

The current state of experimental knowledge on isoelectronic sequences that lie between those presented below (i.e., K-shell, lithium-like, sodium-like, and copper-like ions) is even less complete. A typical example of a current 


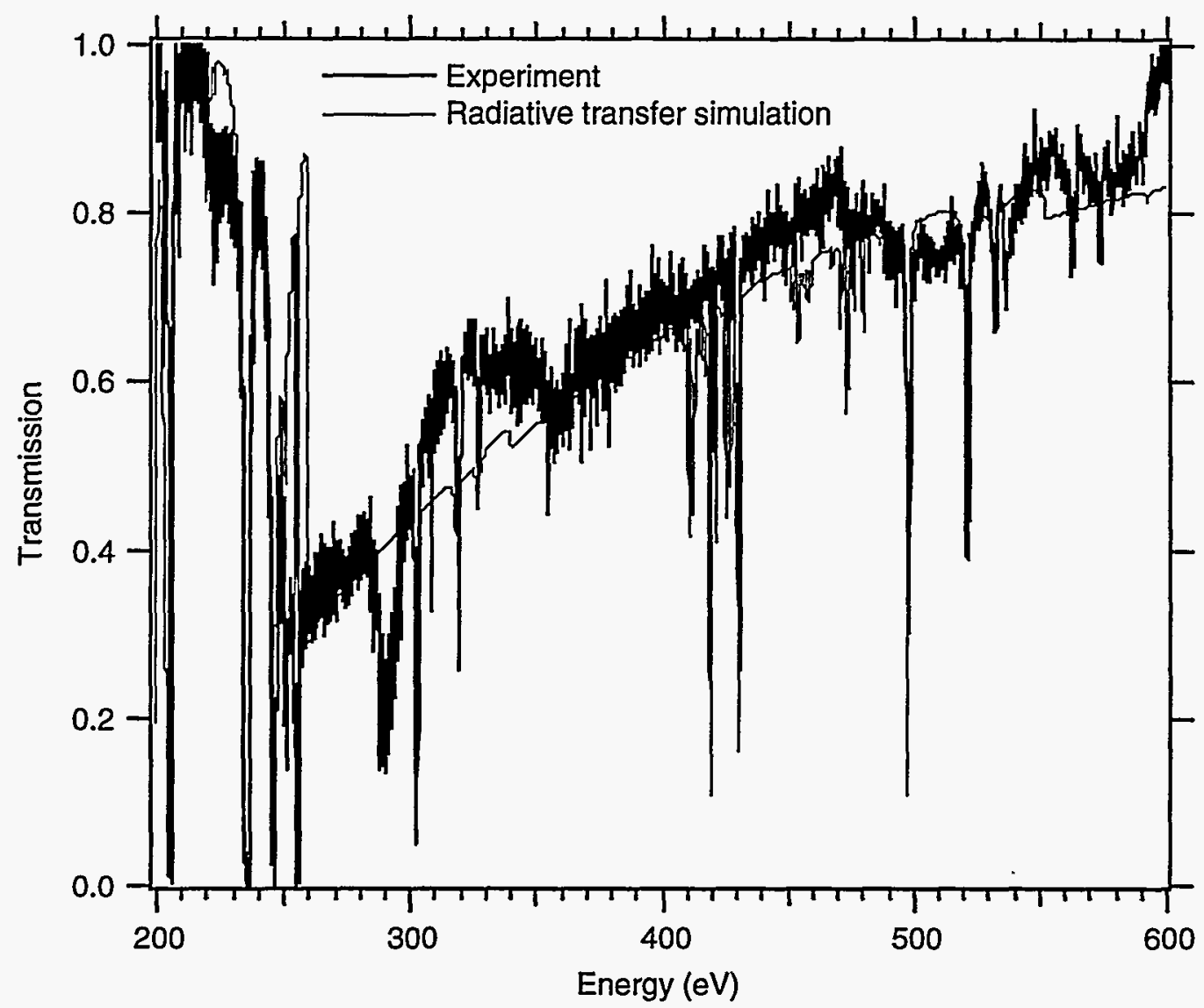

Figure IX-12. Spectrum from absorption of untamped boron nitride. The experiment is the thick solid line; two predictions of the spectra are also shown. The thin line is the prediction of the NLTE radiative transfer code ALTAIR. Note that this sample should not come too far out of LTE, and comparisons have been performed that indicate that this is true.

experimental setup is the dot spectroscopy schematic shown in Fig. IX-1. It is believed that using a source as powerful as the NIF laser with special foam target designs, or using other mechanisms for producing long-scale-length plasmas, can achieve coronal temperatures of $6 \mathrm{keV}$, with eventual production of $10-\mathrm{keV}$ plasmas. Such temperatures will make it possible to probe most elements of the periodic table to any desired degree of ionization.

Observing transitions in higher- $Z$ ions is important for determining the contributions to the transition energies that become significant only at high $Z$. These include QED and relativistic contributions to the transition energies. In addition, electron correlation effects are important at high $Z$. Comparison of observed and calculated transition energies motivates and guides the improvement of the atomic theory of highly charged ions. For example, a great deal of work is presently being done on non-hydrogenic QED theory. Further, the atomic models are input to the codes that simulate opacity and atomic kinetics.

The simplest spectra to interpret, and the easiest transition energies to calculate, are for ions with one weakly bound electron outside a closed shell. These are in effect "one-electron spectra," and they occur for lithium-like ions with 3 electrons, sodium-like ions with 11 electrons, and copper-like ions with 29 electrons. To date, the copper-like sequence has been studied to the end of the sequence (i.e., up to copper-like uranium). The NIF would permit the study of other sequences, such as the sodium-like sequence up to uranium. 


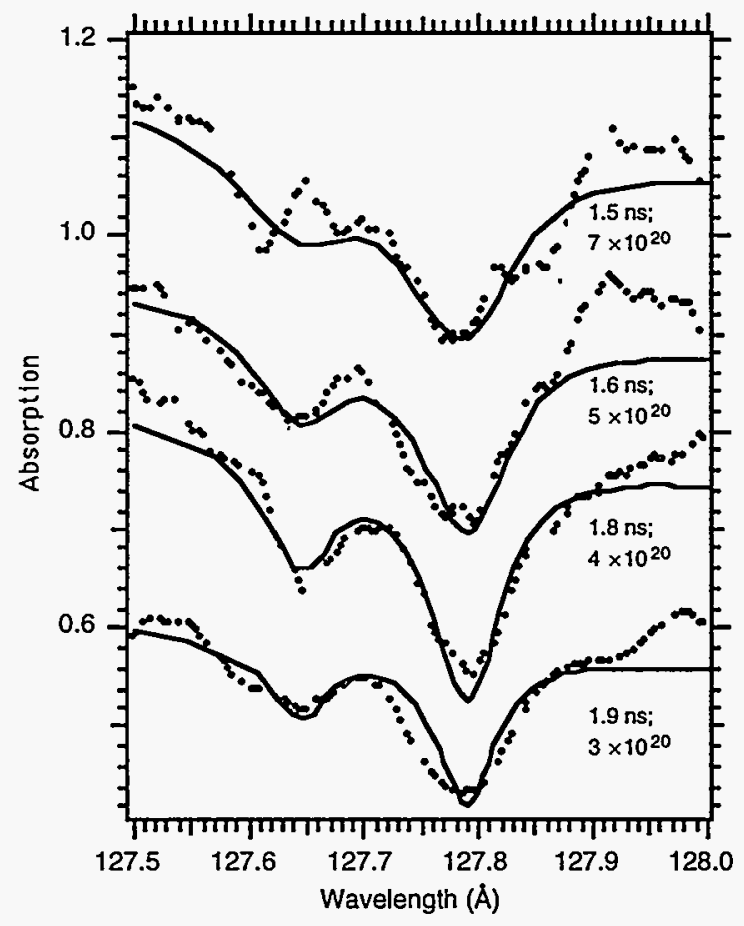

Figure IX-13. Experimental results for absorption of a gold backlight through a plastictamped Teflon sample. The measurement shows the lithium-like fluorine measured along with the theoretical prediction for the line shape. The densities inferred from the measured lithium-like fluorine line shape are also shown.

Isoelectronic sequences that are near the oneelectron sequences are also of interest. Examples are the fluorine sequence with 9 electrons and the magnesium sequence with 12 electrons. Of these two, the fluorine sequence has one electron less than the closed-shell neon-like sequence, and only two transitions are intense in current laserproduced plasmas. Sequences such as the fluorine sequence are used to study the atomic physics of "nearly closed shells." The magnesium sequence has two electrons outside closed shells, and electron correlation effects require detailed calculation. This effect has become an important topic in atomic physics.

The work planned on high-energy lasers must be referenced to the possibilities on other sources of high- $Z$ ions such as the EBIT and Tokamak devices. With regard to EBIT, only two types of transitions are typically intense. These are the resonance transitions that are collisionally excited from the ground state of the ion, and the transitions from the auto-ionizing levels that are resonantly excited by dielectronic recombination. With the EBIT it is not possible to efficiently excite the transitions between excited states. The same limitation applies to Tokamak spectraonly the ground-state transitions are intense.

In addition, although heavy ion accelerators can produce highly charged ions, Doppler shifts complicate the analysis. Also, synchrotron radiation can be used only for the study of cold material, not highly charged ions. Thus, the NIF is necessary for the in-situ and complete study of entire spectra of the highly charged ions that cannot be produced by currently available lasers.

\section{The Transparency Window}

The possible existence of a transparency window in the spectral region between the bound-bound transitions and the formation of the bound-free continuum has been discussed for many years, and the issue is still unresolved. ${ }^{14}$ This possible effect is important because such a decrease in absorption probability can have several serious effects. First, reduction of opacity in this spectral region can lead to overestimates of the opacity of the models used. Second, the recombination rate of ions into the high-lying states could be seriously reduced by such a transparency window, changing the kinetics of the plasma. Finally, modification of the density of states will have an effect on the thermodynamic properties of the plasma. This last effect is due to the direct connection between the density of states and the partition functions, from which all the thermodynamics properties are developed.

To measure radiative properties of this type (that is, those measurements that go beyond spectral identification), the phenomenon of interest needs to be isolated by creating highly constrained plasma conditions. Figure IX-14 shows a schematic of a method that generates a single-density single-temperature plasma for these types of studies. To ensure uniform heating, the experiment shown uses volumetric irradiation with $\mathrm{x}$-rays. The sample is tamped to keep its density and temperature uniform. 


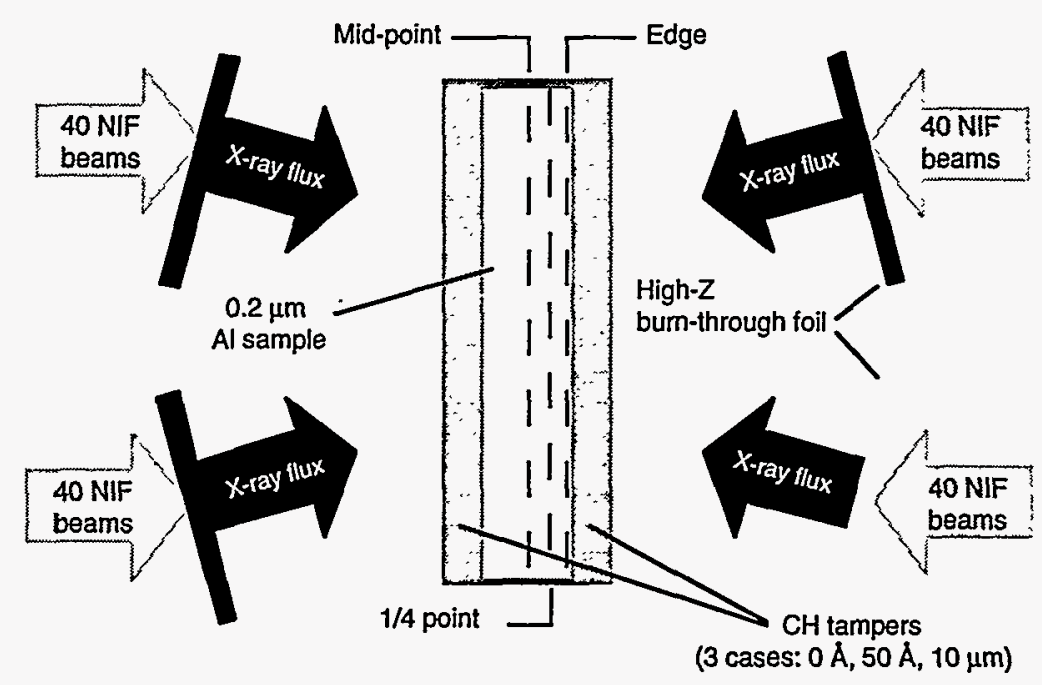

Figure IX-14. Schematic of proposed experiment to obtain uniform temperature and density. To ensure uniform heating, volumetric irradiation using $x$-rays is employed. The tampers are used to keep the density and temperature of the sample at uniform levels.

The requirements for a thin sample and $x$-ray heating imply that little absorption of the $x$-ray energy occurs. The low absorption, coupled with the fact that the sample $x$-rays are produced by laser-plasma generation, indicate that the laser energy must be large to yield substantial plasma heating. Combined with this is the requirement that the sample must be hydrodynamically isolated, which means that laser-plasma creation must occur at substantial distances. The distance further diminishes the coupling and increases the laser energy requirements. Simple calculations using approximately 160 of the NIF beams on gold burn-through foils yield $x$-ray flux that will provide researchers in the area of radiative properties an ideal plasma insofar as it has the correct conditions in which to perform measurements.

Figures $D X-15$ and $D X-16$ show plots of temperature and density as a function of time for samples handled two different ways, one overtamped and one undertamped. Figure IX-15 shows a plot of temperature and density for a 2000- $\AA$ aluminum sample that is overtamped to provide a high degree of uniformity. The figure shows information for the three locations in the sample, the midpoint, the quarter-point (halfway between the edge and the midpoint), and the edge. The overtamping minimizes density and temperature gradients in the sample. Overtamping produces some extreme states of matter for study-for example, note that at 1 ns the temperature is over $65 \mathrm{eV}$ and the density is 0.25 $\mathrm{g} / \mathrm{cm}^{3}$. This single temperature and density point is, of course, attainable in many plasmas; however, the study of radiative properties of samples at this single condition will be unique and requires the capability of the NIF.

Figure DX-16 shows an untamped sample of aluminum for the same thickness, with the gradients in temperature and density indicated. The figure shows information for the three locations in the sample: the midpoint, the quarter-point (halfway between the edge and the midpoint), and the edge. The fact that the sample is untamped leads to an exponential decay of density and the formation of gradients. The outer edge of the sample blows down first, and there is a factor-of-three density gradient between the edge and the middle. The temperature shows that the lower-density outer region is hotter. This is consistent with the conservation of energy density of the sample. Clearly, tamping of the sample (as shown in the previous figure) will assist in the performance of single-temperature and single-density experiments. 


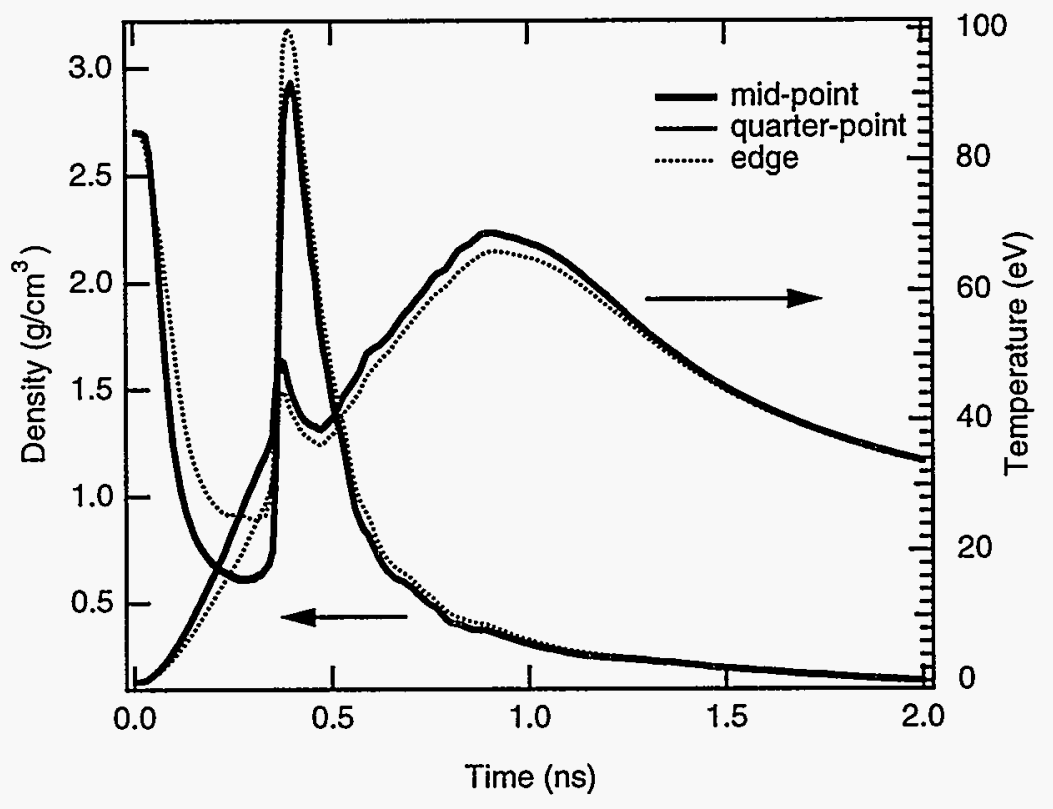

Figure IX-15. Temperature and density vs time for an overtamped sample of $2000-\dot{A}$ aluminum. The plots associated with the left-hand axis show density for the three locations in the sample. The midpoint is shown as a thick solid line; the quarter-point (the position halfway between the edge of the sample and the midpoint) as a thinner line; and the edge as a dotted line. The plots associated with the right-hand axis indicate the temperature. Again, the midpoint is shown as a thick solid line, the quarter point as a thinner line, and the edge as a dotted line. (Note that the quarter-point temperature is not visible as a separate line, as it overlaps the mid-point temperature.) The density and temperature enhancement at $0.4 \mathrm{~ns}$ is due to a shock generated in the rather thick $10-\mu \mathrm{m}$ plastic tamper. The overtamping minimizes density and temperature gradients in the sample. Overtamping produces some extreme states of matter for study-for example, note that at $1 \mathrm{~ns}$ the temperature is over $65 \mathrm{eV}$ and the density is $0.25 \mathrm{~g} / \mathrm{cm}^{3}$.

Transparency (and the other residual effects) are predicted to arise when two conditions exist. The first requirement is a strongly coupled plasma, or a state when the plasma-ion radiator potential energy is greater than the thermal energy, so that the required approximation for the plasma-ion interaction becomes complex. The second is to have an ion described by a nonspherical potential, because for spherical potentials it can be shown that transparency and all the other residual effects do not occur. Thus, in strongly coupled plasmas with complex ions, the transparency effect may be important.

Figure IX-17 shows a calculation illustrating the transparency window position and its effect. The logarithm of opacity is plotted against energy for an aluminum plasma at a temperature of $450 \mathrm{eV}$ and an electron density of $10^{19} \mathrm{~cm}^{-3}$.
The calculations are performed for two cases, one with only Doppler broadening and one with both Doppler and Stark broadening. The region where the ionization potential of the helium-like species occurs is also shown. The Doppler profile spectrum in this region shows a substantial decrease-this is the region of the transparency window.

The existence of a transparency window is in question. The subtlety of the effect indicates that high-quality experiments are required to make a quantitative assessment of the effect, and this will require a NIF-scale facility.

The difficulty here is that to observe a change in the kinetics of the plasma or its thermodynamic properties requires a level of detailed information that is not possible, or thinkable, at the present time. However, there is the possibility 


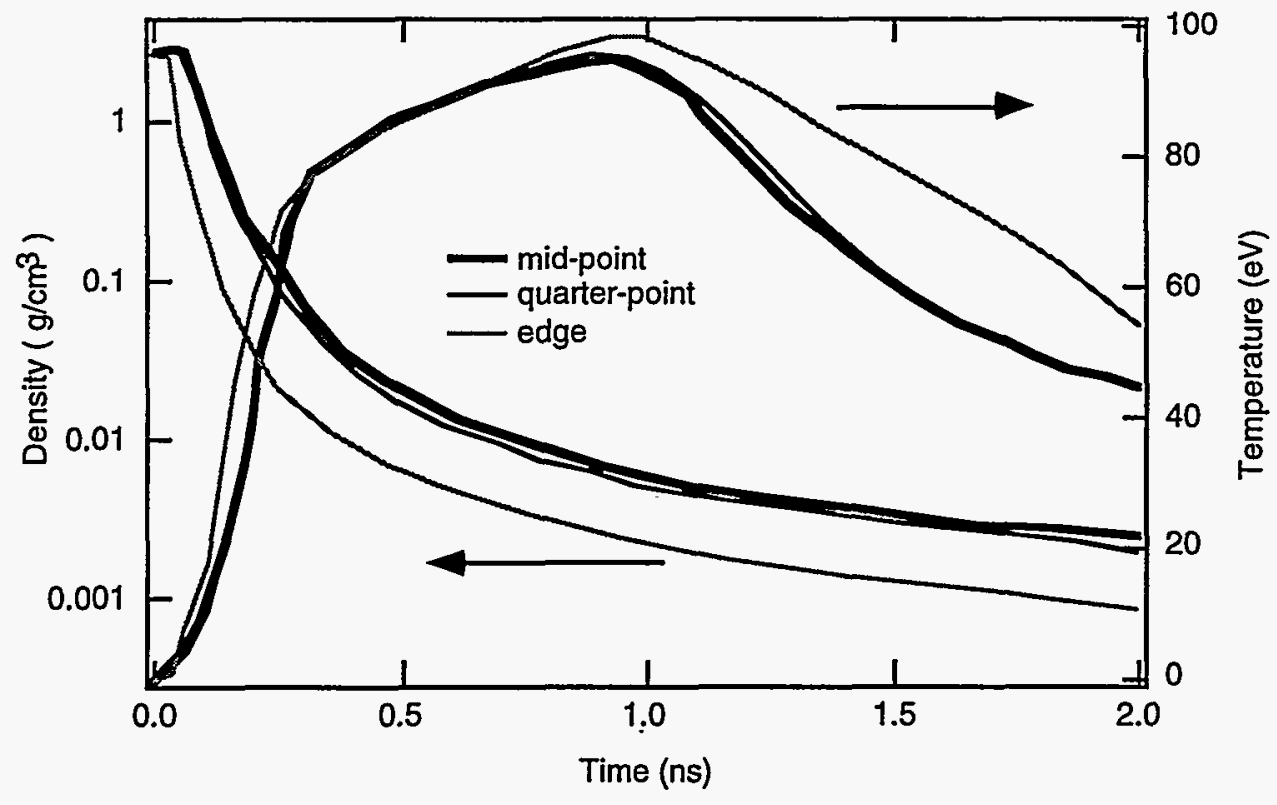

Figure IX-16. Temperature and density vs time for an untamped sample of $2000-\AA$ aluminum. The plots associated with the left-hand axis show the logarithm of density for the three locations in the sample. The midpoint is shown as a thick solid line; the quarter-point (the position half-way between the edge of the sample and the midpoint) as a thinner line; and the edge as a light line. The plots associated with the right-hand axis indicate the temperature. Again, the midpoint is shown as a thick solid line, the quarter point as a thinner line, and the edge as a light line. The fact that the sample is untamped leads to an exponential decay of density and the formation of gradients. The outer edge of the sample blows down first, and there is a factor-of-three density gradient between the edge and the middle. The temperature shows that the lower-density outer region is hotter. This is consistent with the conservation of energy density of the sample. (Figures IX-15 and IX-21 show an overtamped sample and an undertamped sample, respectively.)

that we can probe the region in absorption between bound-bound and bound-free transitions in a specially prepared plasma. First, the effect we are looking for is a subtle change in absorption in a region that has bound-bound and bound-free transitions. Second, the very nature of the strongly coupled plasma problem indicates that the bound-bound transitions will be relatively broad and will form a quasicontinuum. The ability to differentiate these effects and obtain absolute absorption cross sections requires the ability to minimize the gradients of the system. Methods for generating , hot, dense plasmas of this type were discussed in Subsection E and here. Importantly, there has been much effort spent on developing the techniques to perform precision absorption spectroscopy on these kinds of plasmas.
The proposed experimental setup to study the transparency window might look similar to the experimental setup shown in Fig. IX-14. With this configuration, small differences in optical depth (on the order of 0.1 or less) can be measured accurately. However, it is difficult to generate a benign environment that can be probed.

\section{Strongly Correlated Effects}

It is important to make the distinction between the concepts of strongly coupled plasma effects and strongly correlated plasma effects. Strongly coupled plasma effects concern only the relative contribution made by the plasma interparticle Coulombic potentials to the plasma statistical mechanics. The Coulombic potentials 


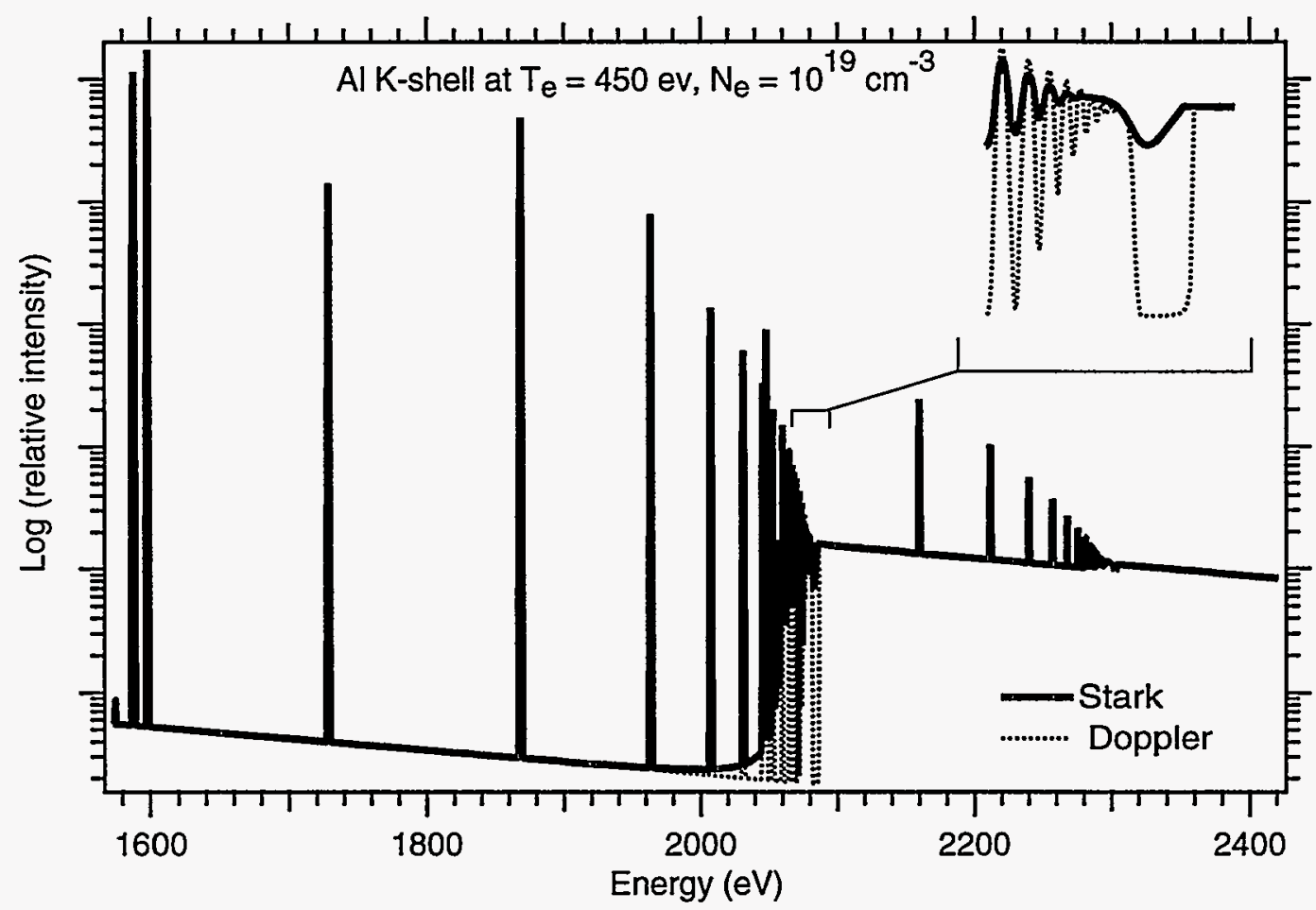

Figure IX-17. Transparency window (calculated) in the spectral region between the bound-bound and bound-free transitions. The logarithm of opacity is plotted against energy for an aluminum plasma at a temperature of $450 \mathrm{eV}$ and an electron density of $10^{19} \mathrm{~cm}^{-3}$. The spectral features arise from the helium-like $1 s^{2}-1$ snp ${ }^{1} \mathrm{P}$ series and hydrogen-like Lyman series 1s-np, which overlap. The calculations are performed with only Doppler broadening (indicated by the grayed line) and Doppler and Stark broadening (indicated by the solid line). The inset shows the region where the ionization potential of the helium-like species occurs. The Doppler profile spectrum in this region shows a substantial decrease-this is the region of the transparency window. Note that for the Stark-broadened transitions, transparency is substantially reduced.

are given by $\mathrm{V} \sim \mathrm{Z}_{1} Z_{2} \mathrm{e}^{2} / \mathrm{r}_{12}$, where the $\mathrm{Zs}$ are the charges on particles and $r_{12}$ is the mean interparticle spacing.

Strongly correlated plasma effects can be schematically defined as the regime in which modification of the interatomic potentials of the ions by other ions in turn substantially modifies effects on radiative properties.

The regions in temperature and density parameter space where strong correlations occur are shown in Figs. IX-18 and IX-19. Figure IX-18 indicates these regions with reference to physical parameter configurations for a pure hydrogen plasma. The figure shows the regions of current experiments. The radiatively heated experiments are shown in the left-hand rectangle, the plasmas arising from spherical implosion in the upper right rectangle, and the planar shock experiments in the lower rectangle.

To indicate the regions that are strongly coupled, the contour where the strong coupling parameter $\Gamma$ is unity is shown with a thicker line; the region below this line is strongly coupled. The strong coupling parameter $\Gamma$ is defined as the ratio of the ion-ion potential energy $\left(Z^{2} e^{2} / r_{0}\right)$ to the thermal energy $(k T) . Z$ is the mean ion charge (here taken to be 1 for hydrogen), and $r_{0}$ is the mean distance between ions [i.e., equal to $\left.\left(3 Z / 4 \pi N_{e}\right)^{1 / 3}\right]$.

The region where the electrons in a hydrogen plasma become degenerate (that is, the condition where the chemical potential is zero) is indicated 


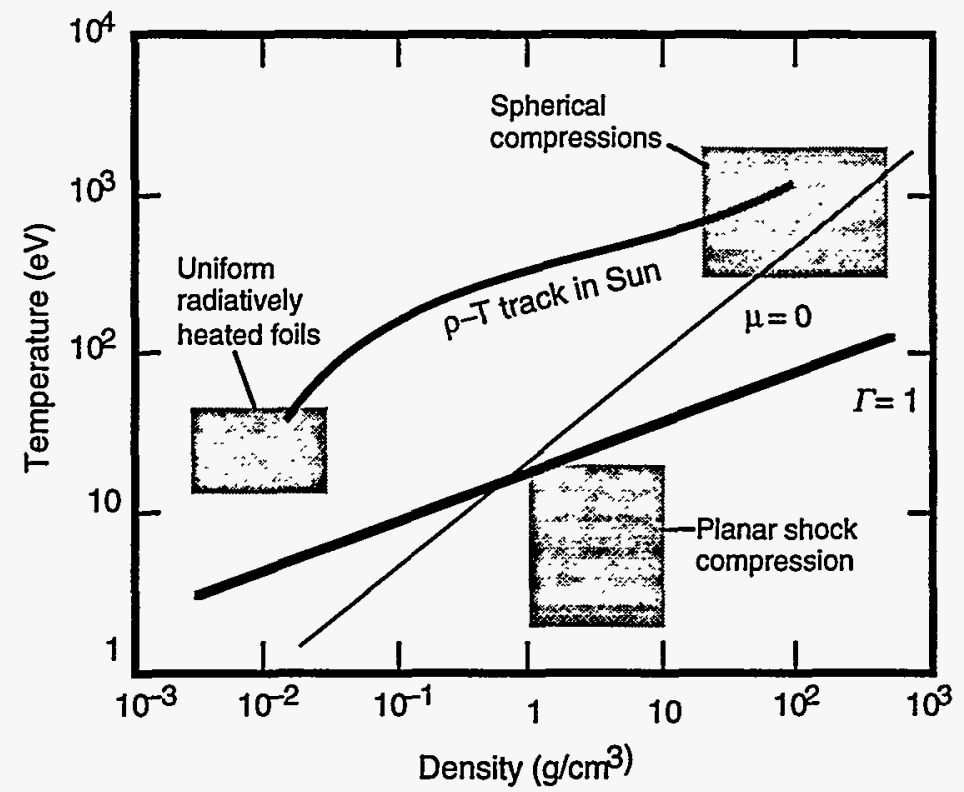

Figure IX-18. Temperature and density parameter configurations for various laser experiments. The regions of current experiments are the radiatively heated experiments (left-hand rectangle), the plasmas arising from spherical implosion (upper right rectangle), and the planar shock experiments (lower right rectangle). To indicate the regions that are strongly coupled, the contour where the strong coupling parameter $\Gamma$ is unity is shown (thick line); the region below this line is strongly coupled. The region where the electrons in a hydrogen plasma become degenerate (that is, the condition where the chemical potential is zero) is indicated by the contour labeled $\mu=0$ (thin line). All regions below this line require treatment of partial or completely degenerate plasmas. Finally, the regions that will be available with the NIF are indicated by the large light-gray area toward the top of the graph.

by the contour labeled $\mu=0$ (the thinner line). All regions below this line require treatment of partial or completely degenerate plasmas.

Finally, the regions that will be available with the NIF are indicated by the large light-gray area toward the top of the graph. It is easy to see that extension of these experiments to conditions of interest in the study of the radiative properties of strongly coupled matter will be greatly enhanced by the advent of the NIF.

Figure IX-19 isolates the temperature and density case for aluminum, showing the effects of ionization in the study of strongly coupled plasmas. The figure defines three regions. The region where classical plasma physics is appropriate is shown in white. In this region Debye-Hückel theory can be used, because there are many electrons in a Debye sphere.

In the dense plasma region (the gray area in the middle), the Debye length is becoming comparable to the interparticle spacing, and high-lying states-the Rydberg states - of one ion can interact with other ions to form band-like states. This yields, among other things, lowering of the ionization potential.

In the high-density-matter region (the dark area to the right of the dotted line), beyond the contour where degeneracy becomes important, three things happen: the plasma now becomes closely associated to a liquid metal; inner-shell states form energy bands; and the ions are densely packed, so distortion from neighboring ions is substantial. The $\Gamma=1$ contour is irregular, which is due to the fact that detailed effects of ionization of the aluminum create changes in the $Z$ and thus in the average potential.

We can use as a measure of strong correlation the amount by which the ionization potential of the ion is perturbed. This is a reasonable measure, in that it points out that strong correlations occur for some principal quantum number in all plasmas. But it also shows that 


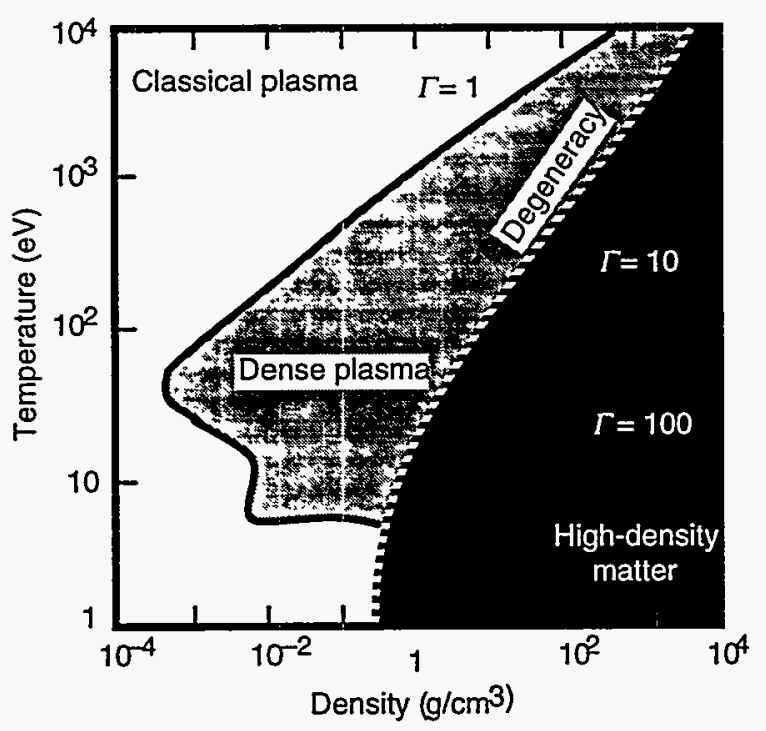

Figure IX-19. Temperature and density diagram for a specific element (aluminum). The diagram shows the effects of ionization on the region of interest to the study of strongly coupled plasmas. Three regions are defined. The region where classical plasma physics is appropriate is shown in white. In this region Debye-Hückel theory can be used, because there are many electrons in a Debye sphere. In the dense plasma region (the gray area in the middle), the Debye length is becoming comparable to the interparticle spacing, and high-lying states-the Rydberg states-of one ion can interact with other ions to form band-like states. This yields, among other things, lowering of the ionization potential. In the high-density-matter region (the dark area to the right of the dotted line), beyond the contour where degeneracy becomes important, three things happen: the plasma now becomes closely associated to a liquid metal; inner-shell states form energy bands; and the ions are densely packed, so distortion from neighboring ions is substantial. The $\Gamma=1$ contour is irregular, which is due to the fact that detailed effects of ionization of the aluminum create changes in the $Z$ and thus in the average potential.

modification of the radiative properties may not be substantive. This means that, for example, the depression of ionization potential, which is discussed below, can be well approximated by simple means, and is not formally associated with strong correlations.

On the other hand, the formation of spectral features due solely to the correlations (e.g., quasimolecular formation) is considered an effect of strong correlation. Further, we note that it is simpler to get a strongly correlated plasma than to get a strongly coupled plasma. For example, the plasmas in stellar interiors are very dense, and while their plasma properties are calculable with weak coupling, or Debye, theories, strong correlations will exist because the overlap of the interatomic potentials is large.

An example of these strongly correlated effects is the transient quasi-molecular effect giving rise to satellite lines. There are several suggestions in the literature concerning these satellite lines. First, there is the prediction of a molecular resonance on the far wings of the Lyman $\alpha$ transition of hydrogen in a plasma, due to strong correlation with other hydrogen atoms. ${ }^{15}$ The upper limit of density for such effects can be estimated by considering that when the orbital of interest is at the mean ion sphere radius, quasi-molecular formation will occur. For the $n=2$ state of a hydrogenic species of atomic number $Z$, the orbital radius is $r=6 / Z a_{0}$, so that the limiting electron density would be $Z^{4} \times 7.46$ $\times 10^{21} \mathrm{~cm}^{-3}$. The quasi-molecules would form at densities below this, but there would have to be a probability distribution associated with the dynamics of the system; at higher density the plasma would form band structures.

Second, this strong correlation effect on radiative property has been numerically studied for a pure hydrogen plasma. ${ }^{16}$ This study does show that it is possible to get groupings of atoms in which the charge distribution is highly distorted, resembling molecular clusters. However, these clusters are not necessarily bound. Static calculations performed on argon in a hydrogen medium yield configurations of atomic positions from a molecular dynamics calculation. Subsequent electronic structure calculations over many configurations show that bands and line broadening form in a nonperturbative manner, and the averaged results form one composite transition. Preliminary work for these static calculations indicate that the $n=3$ 
manifold of argon is heavily influenced by the hydrogen band.

Third, there have been claims of satellites, assumed to be quasi-molecular in origin, that form in laser-produced plasmas. The effect was tentatively observed in the spectra from laser plasma produced by irradiating a lithium fluoride planar target. This was done by analyzing the Lyman $\beta$ transition in fluorine with an emphasis on the short-wavelength wing. The results provided small features that may be attributable to quasi-molecular resonances. ${ }^{17}$ These observations are not sufficient to prove the existence of the satellite lines and do show the difficulty with current experimental techniques.

The performance of experiments on plasmaemitter radiative properties will require a more idealized setup than employed in the direct-laser planar target irradiation experiment. The effects sought are quite subtle, so working as close to a gradientless case as possible will improve the chances of success. To have at the same time both a uniform plasma and a plasma in an extreme state requires the expenditure of a large amount of energy.

The highest level of success would be achieved by using a well-structured shock-based system. For example, an adiabatic or isentropic shock could be produced using a tailored $x$-ray driving source. This may allow the possibility of compressing material to 10 times denser than liquid density. The difficulty will be in maintaining a low temperature in the shockcompressed region and devising a diagnostic complement that can observe the effect. There are further discussions of shock compression in Section VI, Material Properties, Subsection C.

\section{Plasma Spectroscopic Topics}

\section{Spectral Line Shifts, Level Shifts, and Continuum Lowering}

The measurement of spectral line shapes, including spectral line shifts, has been a topic of continuing interest because of the diagnostic capability provided by this non-interfering probe of plasma conditions. This has meant that for each new plasma source there is a new and challenging series of theoretical developments that must follow to provide the diagnostic information.

Thus, on the one hand, experimental development has fueled the need for line shape modeling. On the other hand, the line shape, width, and shift, and the formation of the line series leading to the bound-free limit contain some of the most fertile ground for exploring the general area of radiator-plasma interactions. In this way the underlying plasma-electron density and temperature are important, but so are the more subtle facets of the plasma, such as fluctuation levels, composition of the plasma, and non-equilibrium velocity distributions.

All of these facets can provide important signatures in the line spectrum and thereby provide a method of testing theoretical statistical mechanics and kinetics formulations, which means the line spectrum has both diagnostic and intrinsic interest. Figure IX-20 shows an example of the competing effects of line shapes, line merging, and continuum lowering for a K-shell aluminum sample at several densities and a single temperature $(450 \mathrm{eV})$.

The spectral features arise from the heliumlike $1 s^{2}-1$ snp $1 \mathrm{P}$ series and hydrogen-like Lyman series 1s-np, which overlap. In the lowest density case (at $10^{19}$, lower plot), the line spectrum has many states leading to the bound-free continuum. In the intermediate density case (at $10^{22}$, middle plot), the lines are substantially broadened and there is a merging of levels, due to the broadening near the continuum edge. At the highest density (at 1025, top plot), the spectrum shows the ionization potential to be depressed by $250 \mathrm{eV}$, and thus only the states with principal quantum numbers equal to 2 still exist.

It can be seen from these results that singledensity, single-temperature samples will be critical to evaluating the complex interaction of the related mechanisms of line broadening, ionization potential depression, and line merging. The NIF holds out the promise of attaining these plasma conditions in the ideal experimental conditions.

One aspect of the line spectrum that is opened to study is the evaluation of line shifts. Line shifts are relatively small compared to line 


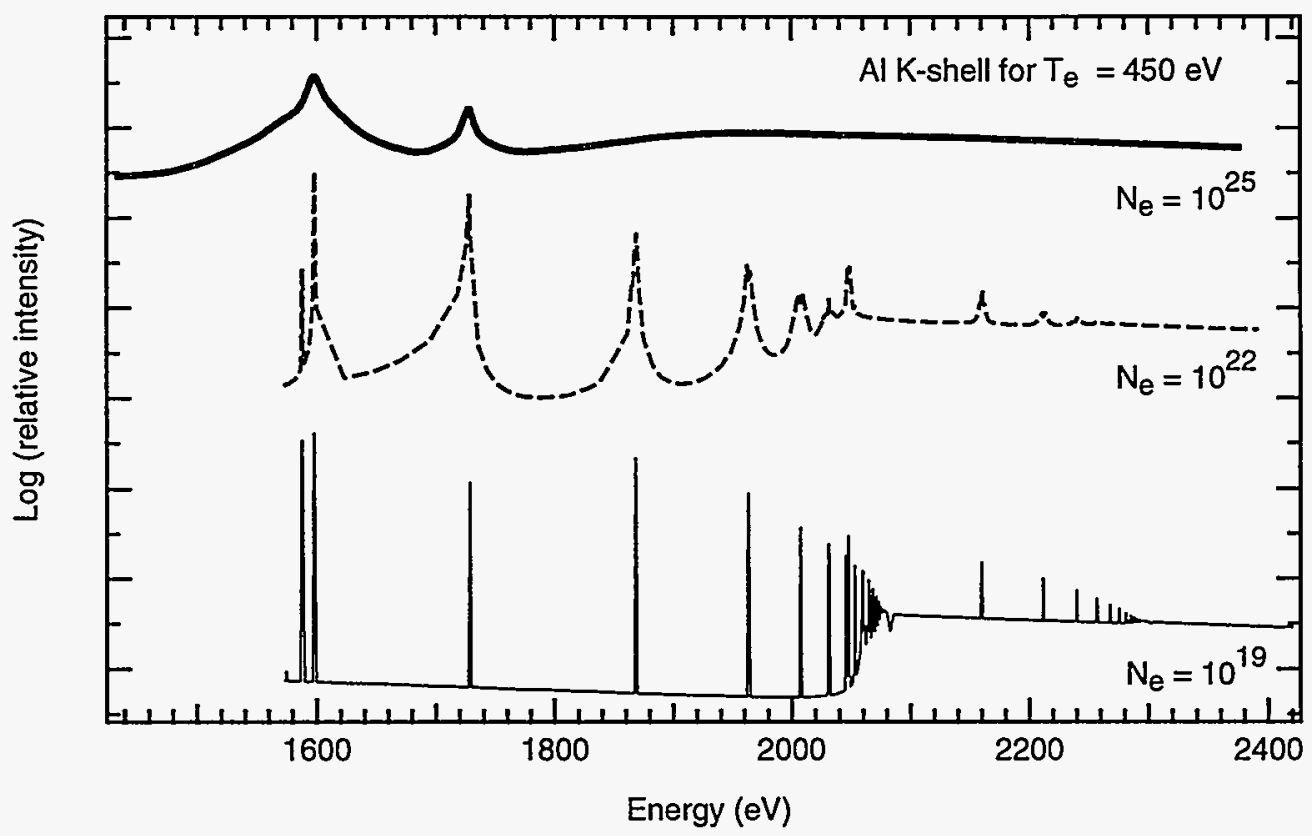

Figure IX-20. Effects of line shapes, line merging, and continuum lowering. The logarithm of the relative intensity of the aluminum K-shell transitions is plotted against energy for three plasma conditions-three different electron densities at an electron temperature of $450 \mathrm{eV}$. The spectral features arise from the helium-like $1 s^{2}-1 s n p{ }^{1} P$ series and hydrogen-like Lyman series $1 s-n p$, which overlap. In the lowest density case (lower plot, $N_{e}=10^{19} \mathrm{~cm}^{-3}$ ), the line spectrum has many states leading to the bound-free continuum. In the intermediate density case (middle plot, $\mathrm{N}_{\mathrm{e}}=10^{22} \mathrm{~cm}^{-3}$ ), the lines are substantially broadened and there is a merging of levels, due to the broadening near the continuum edge. Increased plasma density has depressed the continuum edge by $25 \mathrm{eV}$. At the highest density (top plot, $N_{e}=10^{25} \mathrm{~cm}^{-3)}$, the spectrum shows the ionization potential to be depressed by 250 $\mathrm{eV}$, and thus only the states with principal quantum numbers equal to 2 still exist.

width, and are therefore difficult to measure and extremely difficult to calculate. No significant advance, in terms of a theory, has been made on the calculations of the spectral line shifts since the first formulations of the standard theory were developed in the late 1950s by Baranger and Griem. 18

The shapes of many lines have still not been quantified, because there have been very few high-energy-density experiments where supporting data on the temperature and density of the plasma has been measured. To date there are, for example, no experiments where the density and temperature have been measured with sufficient accuracy in any plasma with a density higher than those probable by Thomson scattering. Thus, although we have for two decades diagnosed laser-plasma densities using line widths, these have never been independently verified. The difficulty in obtaining singledensity, single-temperature plasma is discussed above. The main point is that hot, dense plasma is highly transitory and usually has large gradients. These hot, dense plasmas do not easily lend themselves to the production of highprecision measurements.

The situation becomes even more complex as we attempt to study the entire line series with its associated continuum. The various processes that arise as the series limit is approached are line broadening, line merging, and ionization potential lowering. To distinguish these is very difficult, but experiments that allow for a controlled plasma environment may be able to solve some of theses problems. As indicated in Subsection E, Radiative Transfer, under Non-LTE 
Radiative Transport, single-temperature, singledensity gradient data may allow us to separate the effects of various mechanisms.

We note further that the various processes are in fact all due to the same perturbative mechanisms-the plasma particle fields cause the broadening and shifting of a line, while at the same time they change certain orbitals from bound to free. It is the interplay among these various physical manifestations that forms the nexus for our understanding of radiator-plasma interaction.

We note the fact that there is no complete theory describing the broadening of a boundbound transition as it goes over to a bound-free transition. From a purely phenomenological view, what happens is that the bound-bound resonance must continuously broaden in energy space while the change to a bound-free resonance is made. A schematic approach to this behavior is discussed by Liberman. ${ }^{19}$

To measure an effect of this type requires an experiment that would have observably large ionization-potential depression and substantial line broadening. These two effects are largely complementary in that these both increase directly with electron density. Further, to be able to isolate the line broadening and the transition into the continuum from the effects of line merging (i.e., the Inglis-Teller limiting case), we would like to go to high density and high $Z$ in order to depress the ionization potential to a low enough principal quantum number to allow the broadened spectral lines to be distinguishable (i.e., not merged).

Such experiments have been possible in the core emission of gas-filled microspheres imploded by both direct and indirect laser irradiation. ${ }^{20}$ However, these experiments indicate that the technique of using implosion cores is not sufficient for the task. Although the experiments provide indications of the various critically interesting effects and an interesting set of possible explanations, the data is not of sufficient quality to validate any particular theory.

We note that the development of better diagnostics and smoother implosion-core densities may be the solution, but these would require the increased energy of the NIF. The implosion dynamics that are now available will not allow adequate constraining of the problem. This is simply because the system is driven to peak densities for very short times, and the gradients thus produced are large. This is unacceptable for spectroscopy of the details of the line shape, line merging, and ionization potential depression processes. On the other hand, using somewhat lower- $Z$ elements than argon as fill gases may provide some amelioration of the steep density effect by permitting the study of the interplay of the various line shape effects at a lower density.

\section{Ion Dynamics}

The role of the dynamics of ions in the formation of spectral lines in plasma has been a topic of intense investigation for the last twenty years. Much effort has been expended since the experimental verification that the Lyman $\alpha$ transition of neutral hydrogen was seriously affected by the dynamics of the ions, forcing modification of the standard theory that assumes quasi-static ions. The fact that this discovery came at a time when ionic emitter line profiles started to become ever more available from laserproduced plasma experiments led to much work on the ion dynamics of spectral lines that arise from ionic emitters.

However, to date there are no experiments that can be used to provide a serious test of the theories for hot, dense plasmas. Verification of the ion dynamics contribution is of critical importance for two complementary reasons. First, the use of spectral line broadening may be seriously compromised if the ion dynamics seriously change the widths of the spectral. Secondly, even in those cases where the ion dynamics are negligible, it is only possible to know this by access to a verified model.

To study ion dynamics requires the same type of uniform plasma conditions that are required by the study of plasma-emitter radiative properties, and the discussion on that subject should be consulted. Additional considerations for these experiments require observation of line profiles from the same ionic radiator emitting at the same plasma conditions but from plasmas with different ion perturber masses. The use of 
implosions is attractive from the point of view that hot, dense conditions can be reached.

However, both the gradients in these implosions and the difficulty in obtaining temperature and density histories with different atomic species in the fill are unsolved problems. On the other hand, this area is ripe for investigation. Alternatively, the tamped-sample-type plasma may be the solution, but reaching the correct conditions will definitely require NIF-size experiments.

\section{Continuum Measurements}

The problem of providing continuum measurements is similar to the difficulty of measuring the absolute values of the continuum in any given experiment. The spectral localization of bound-bound or even bound-free edges provides a great simplification in separating out signal from background compared to the determination of continua. This is true in all plasmas, but when we add the hot, dense and/or strongly coupled (and correlated) effects we are faced with, a daunting problem arises in how to proceed with measurement. While it is clear that interesting processes, such as strongly correlated scattering cross sections, can come into play in the formation of continua, what is not clear is how to proceed.

The experimental setups discussed above (e.g., Fig. IX-14) are not sufficient in themselves to provide good continuum measurement. The additional constraint is that the material surrounding the material of interest, whether it be a tamped sample or an implosion, must have continuum contributions that are either small or completely quantified. It is easy to envision keeping the contributions small. In such cases the density of the material of interest would have to be increased relative to, for example, a sample prepared for the line shape measurements, where the optical depth in the lines would be kept small. However, with increased thickness come increased plasma gradients, and the problems of volumetric heating are exacerbated. This makes the energy-rich environment of the NIF even more desirable.

In this context it would be appropriate to note that the important aspects of bound-free edges and continuum processes are at some level the same, with the formation of free-free continua being an additional part of the puzzle. Further, the study of auto-ionization processes and how these behave in dense plasmas is also of great interest, not only in the study of plasmaemitter radiative properties, but also in the area of kinetics and radiative transfer.

\section{Population Kinetics}

The study of the dynamics, or kinetics, of an atom immersed in a plasma is at the center of the ability to model time-dependent behavior of atoms in plasma. For the present discussion we assume that radiative transfer is not an integral part of those kinetics. (Situations where the radiative transfer is important are discussed in the next section, Radiative Transfer and Line Formation.)

Even when the radiative properties have been understood, the kinetics of the plasma are still in question. The reason is quite simple; in order to model population dynamics, the rates (or more accurately, the cross sections) for all the processes involved must be well known. Further, in those cases where non-Maxwell-Boltzmann distributions are important, the kinetics behavior would have to be accompanied by a

measurement of the velocity distributions in lieu of temperature measurements.

Knowing the rates and measuring velocity distributions is an extremely difficult task and is not made simpler by choosing to study the behavior of the atom in hot, dense media. Although the densities may be high and give rise to plasmas that rapidly thermalize the velocity distributions, the rates for ionization and recombination are also rapid. Thus the dynamic ionization balance can be dictated by nonthermal distributions. This will be particularly important for experiments with short-pulse, intense laser-solid matter interactions.

In the laser intensity regimes of $10^{15} \mathrm{~W} / \mathrm{cm}^{2}$ and above, which will be realized for subpicosecond pulses, the electron distribution that is produced in the laser-matter interaction is highly non-thermal. This non-thermal electron distribution is created through non-local transport of electrons from the laser interaction region to a region of higher density, where the 
electron temperature (as measured by the electron subsystem energy content) is predicted to be up to ten times higher than the local ion temperature. Ionization, therefore, is of great interest. However, the more mundane plasmas (at least by these standards) created by volumetric $x$-ray heating or implosion of a microsphere have the same interesting problems.

Further, we note that at these high intensities the laser electric field equals or exceeds the binding field in many atoms. In such cases, the laser field must be included non-perturbatively in the calculation of atomic and molecular properties. The laser field also affects scattering processes. For example, Feshbach-like resonances can be induced even in such a simple system as electron + proton, which without the field can have no resonances. Of course, these studies of intense field effects are in their infancy, but they will have to be addressed at some stage in future plasma models and experiments.

To map the kinetics of atoms in a plasma requires a rich set of diagnostics that can provide spatial, temporal, and spectral coverage of the same event. This requires not only a wellcharacterized plasma, but a resource base that is beyond the level that can be brought to bear by an independent effort without the support of a facility of the scale of the NIF. Further, primary information on the plasma character must be available to corroborate the results. Again, the ability to perform temperature and density measurements is critical to the success of these experiments.

We note here that the numerous studies to date on the kinetics of plasma formed by direct illumination (for example) have left many unresolved issues. This is not for lack of desire, but mostly for the lack of both resources and plasma-generating devices that would allow critical investigations. The NIF would provide such a research environment.

Below we will discuss one type of experiment that is motivated by astrophysical considerations. However, the range of areas in which kinetics is of importance is much broader. The most important factor is that with the plasmaproduction capability and the diagnostic complement that the NIF will offer, the complex mix of processes relevant to the kinetics problem can be attacked in a coherent manner.

\section{Radiation Transfer and Line Formation}

The study of the formation of spectra for time-dependent hot, dense media is the culmination of all the topics that have been discussed above. First, the radiative properties are required as initial input, and second, the kinetics model must be verified by experiment so that one can isolate the radiative transfer effects. With these two in place, it is possible to study the non-local effects that arise in the formation of the spectrum.

For these effects one must solve the radiative transfer equation in tandem with the equations for the population dynamics. Because each affects the other, the problem is a compounding of previous investigations. Here, the regimes where the radiation transfer will be severely stressed will also be challenging for experiments. Part of the problem is the need to quantify the sources of the radiation field and radiation matter coupling. The rest of the problem is to create high-opticaldepth, radiatively dominated transitions. These are extremely difficult in the present generation, and the NIF will certainly be required to investigate the regime thoroughly.

Fluorescence has been used in the study of kinetics to probe the movement of population around a network of levels, thus allowing the direct study of the redistribution of population. However, the difficulty in generating efficient photopumps is dependent on the radiative transfer process. For hot, dense matter, pumpprobe experiments are beyond the realm of present-day laser systems. There may be the chance line coincidence between a soft $x$-ray laser and a transition of interest, but other than this bit of serendipity, pump-probe experiments will have to wait for the increased photon densities available with the NIF laser. Then it will be possible to use filtered backlights of sufficient brightness to pump line transitions in hot, dense matter.

A schematic experiment would look roughly as follows. We take a large low- $Z$ tamped sample of mid-Z element. The sample is irradiated with an $x$-ray source that is filtered to ensure that there is no surface deposition due to critical density 
effects. Next, with the tamping controlling the gradient scale-length in the problem, we perform absorption and emission studies, in different spectral regimes. It should be possible to obtain temperatures of $>100 \mathrm{eV}$ for centimeter-size samples. The limiting factor here is that hydrodynamic isolation is absolutely required to ensure that blast wave and debris do not compromise the results.

Next, a photopump of measured temporal, angular, and spectral content is used to pump the plasma. The technique can now be used to observe line formation in the chosen transitions.

It would then be possible to explore the control of velocity gradients, and more generally the interaction of the radiative transfer and the hydrodynamics of the plasma. We note again that this is a regime that is completely beyond current capability. Measurement of the velocity fields in addition to the other parameters required would be impossible in the current experiments.
Figure IX-21 shows a calculation of the behavior of an undertamped aluminum sample with a modest gradient. Undertamping leads to controllable density and temperature gradients in the sample. Obtaining these conditions in a controlled manner requires the energy of a NIF. This undertamped case, together with the overtamped and untamped cases shown in Figs. IX-15 and IX-16, indicate the degree of control that the $\mathrm{X}$-ray heating flux will provide to the users of the NIF.

We note that with hot, dense matter, for the velocities to be of concern, they must be larger than the line width, which we assume is given by the Doppler width of the transitions of interest. Thus, for example, for a $100-\mathrm{eV}$ plasma with $Z \sim 16$, velocities in excess of $5 \times 10^{6} \mathrm{~cm} / \mathrm{s}$ would be required. Such velocities are easily obtained from a free expanding surface and can be associated with untamped surface expansion.

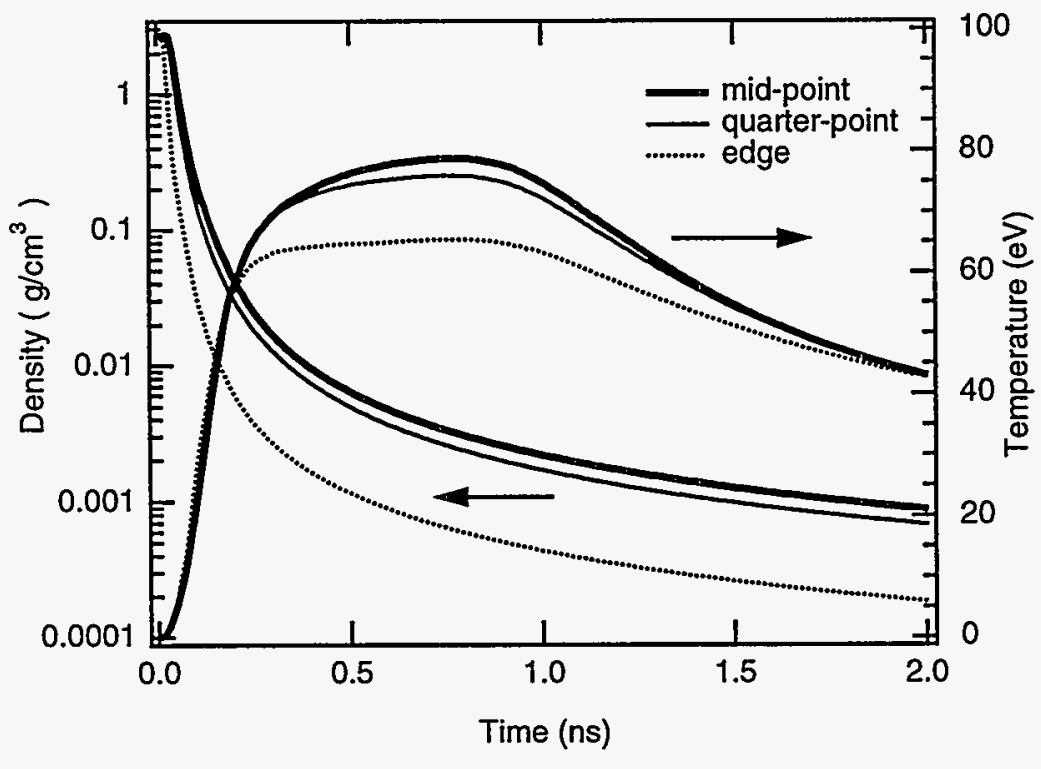

Figure IX-21. Temperature and density vs time for an undertamped sample of $2000-\dot{A}$ aluminum. This represents the time evolution of a plasma that can be used to study radiative transfer and line formation in a controlled system. The plots associated with the left-hand axis show density for the three locations in the sample. The midpoint is shown as a heavy solid line; the quarter-point (the position half-way between the edge of the sample and the midpoint) as a thinner line; and the edge as a dotted line. The plots associated with the right-hand axis indicate the temperature. Again, the midpoint is shown as a thick solid line, the quarter point as a thinner line, and the edge as a dotted line. Undertamping leads to controllable density and temperature gradients in the sample. (Figs. IX-15 and IX-16 show an overtamped sample and an untamped sample, respectively.) 


\section{Strong Magnetic Field Effects}

It is straightforward to deduce from Ampere's law that hot, dense plasmas with small scale lengths might contain strong magnetic fields, with $B>10^{6} \mathrm{G}$, and indeed this is the case. Flux compression yields megagauss fields as a matter of course in explosive pinch experiments, and suprathermal electron currents arising from laser plasmas can produce fields exceeding 100 megagauss. 21,22

The behavior of matter in such strong magnetic fields continues to pose difficult questions in atomic/plasma physics, with importance for astrophysical as well as laboratory applications. For instance, some white dwarf stars have megagauss surface fields, and gigagauss fields are usually ascribed to neutron stars. Strong, primordial fields may also exist in the interiors of giant planets and in the cores of normal stars, including the sun, but their presence cannot be deduced with our primitive understanding of how equations of state and transport coefficients are modified by large B-values.

Recent years have witnessed considerable progress in understanding certain effects of the huge $10^{12}$-G fields expected in neutron star crusts. ${ }^{23}$ However, the fact that Coulomb interactions are relatively unimportant there actually makes it easier to develop good theoretical models for this regime than for the intermediate $10^{7}$ - to $10^{9}-\mathrm{G}$ regime, where magnetic and Coulomb effects are comparable ${ }^{24}$ and perturbation methods are reliable. The suite of diagnostics expected to be available for studying high-energy-density plasmas with the NIF will provide a unique opportunity to probe specific issues related to intensely magnetized plasmas, such as:

- How do the strong B-fields modify plasma ionization balance?

- How important are the anisotropic pressure effects that arise when $B^{2} / 8 \pi$ is comparable to the gas pressure?

- What is the effect on transport coefficients of non-spherical atomic charge distributions that occur when $\mathrm{B}>10^{8} \mathrm{G}$ ?
- Is it possible to use Stark broadening by motional (i.e., $\mathrm{v} \times \mathrm{B}$ ) electric fields as a reliable diagnostic of the strong $B$-fields in plasmas?

\section{G. References}

1. P. G. Burkhalter et al., Phys. Fluids 26, 3650 (1983).

2. J. F. Seely et al., "Zn-like 4141 '," submitted to Phys Rev. Lett.

3. J. F. Seely et al., "Ho XXXIX density sensitive lines," accepted to Phys. Rev. E.

4. See, for example, J. Bauche, C. Bauche Arnoult, and M. Klapisch in Advances in Atomic Physics, D. R. Bates, Ed. (Academic Press, New York, 1988).

5. B. K. Young et al., Phys. Rev. Lett. 61, 2851 (1988).

6. R. Majoribanks et al., Phys Rev. A 46, R1747 (1992).

7. W. Hsing, B. MacGowan et al., private communication.

8. R. W. Lee, AIP Conf. Proc., E. Marmar and J. Terry, Eds. (American Institute of Physics, New York, NY, 1992), Vol. 257.

9. T. S. Perry et al., Phys. Rev. Lett. 67, 3784 (1991).

10. B. K. Young et al., Phys. Rev. Lett. 62, 1266 (1989).

11. L. Da Silva et al., SPIE Proceedings (SPIE) Vol. 2012, page 158.

12. T. S. Perry, Phys. Rev. Lett. 67, 3784 (1991).

13. D. R. Kania et al., Phys. Rev A 46, 7853 (1992) and C. A. Back et al., JQSRT 51 (1994).

14. Some theoretical discussions of the transparency window include: $L$. G. Dyachkov et al., JQSRT 44, 123 (1990) and B. G. Berkovsky et al., J. Phys. B 26, 2475 (1993). Some experimental work includes: V. E. Fortov et al. in Strongly Coupled Plasmas, S. Ichimaru, Ed. (Elsevier, 1990), p. 571; and Popovic \& Dodevic, in Strongly Coupled Plasmas, H. Van Horn \& S. Ichimaru Eds. (University of Rochester Press, 1993), p. 273.

15. S. J. Rose, Journal de Physique Coll. Ser. C 8 (1982); using methods of D. R. Bates, MNRAS 112, 40 (1952); D. Salzmann and J. Stein, Phys. Rev. A 45, 3943 (1992). 
16. I. Kwon, L. A. Collins, J. D. Kress,

N. Troullier, and D. L. Lynch., Phys. Rev. E 49, R4771 (1994).

17. E. LeBoucher-Dalimier, A. Poquérusse, P. Angelo, I. Gharbi, and H. Derfoul, JQSRT 51, 187 (1994).

18. See the references in H. R. Griem, Spectral Line Shapes from Plasmas (Academic Press, New York, 1972).

19. D. Liberman and J. R. Albritton, JQSRT 51, 197 (1994) and the references therein.

20. B. A. Hammel, C. J. Keane, M. D. Cable, D. R. Kania, J. D. Kilkenny, R. W. Lee, and R. Pasha, Phys. Rev. Lett. 70, 1263 (1993); and C. F. Hooper, D. P. Kilcrease, R. C. Mancini,
L. A. Woltz, D. K. Bradley, P. A. Jaanimagi, and M. C. Richardson, Phys. Rev. Lett. 63, 267 (1989).

21. N. Miura and F. Herlach, in Strong and Ultrastrong Magnetic Fields and Their Applications, F. Herlach, Ed. (Springer, Berlin, 1985).

22. S. C. Wilks, W. L. Kruer, M. Tabak, and A. B. Langdon, Phys. Rev. Lett. 69, 1383 (1992).

23. P. Meszaros, High-Energy Radiation from Magnetized Neutron Stars (U. Chicago Press, Chicago, 1992).

24. E. P. Lief and J. C. Weisheit, Contrib. Plasma Phys. 33, 471 (1993). 